\author{
Universidade de São Paulo \\ Instituto de Física
}

\title{
Estudo da Fusão Nuclear e do Potencial de Polarização para Sistemas de Íons Pesados
}

Gustavo Pires de Almeida Nobre

Orientador: Prof. Dr. Luiz Carlos Chamon

Tese de Doutorado apresentada ao Instituto de Física da Universidade de São Paulo para obtenção do título de Doutor em Ciências.

Banca Examinadora:

Prof. Dr. Antonio F. R. T. Piza - IFUSP

Prof. Dr. Edílson Crema - IFUSP

Prof. Dr. Luiz Carlos Chamon - IFUSP

Prof. Dr. Jesus Lubian Rios - UFF

Prof. Dr. Sérgio J. B. Duarte - CBPF 
FICHA CATALOGRÁFICA

Preparada pelo Serviço de Biblioteca e Informação

do Instituto de Física da Universidade de São Paulo

Nobre, Gustavo Pires de Almeida

Estudo da fusão nuclear e do potencial de polarização para sistemas de íons pesados São Paulo, 2007.

Tese (Doutorado) - Universidade de São Paulo. Instituto de Física - Departamento de Física Nuclear

Orientador: Prof. Dr. Luiz Carlos Chamon

Área de Concentração: Física

Unitermos: 1. Fusão nuclear; 2. Espalhamento

elástico; 3. Canais acoplados; 4. Modelos teóricos.

USP/IF/SBI-040/2007 
Ao meu pai, físico, exemplo de perseverança. 
Felix Qui Potuit

Rerum Cognoscere Causas 


\section{Agradecimentos}

Ao meu orientador Prof. Dr. Luiz Carlos Chamon, sou grato pela excelente orientação e por ter tornado todo o desenvolvimento deste trabalho uma atividade bastante agradável.

Aos meus pais, Sérgio A. de A. Nobre e Esperança E. P. de A. Nobre, e irmão, Henrique P. de A. Nobre, pelo convívio e pelo constante apoio e incentivo à minha educação, em especial ao meu pai, com quem sempre pude discutir inúmeros assuntos, ligados a Física ou não.

À minha noiva Ana Elisa Polloni por ser tão especial e por ter sempre estado do meu lado, transformando sempre os momentos mais difíceis nos momentos mais felizes da minha vida.

Ao meu tio Hermon Antonio de A. Nobre pelas primeiras aulas de Física, vinte anos atrás.

Ao Prof. Dr. Brett V. Carlson pelas sempre pertinentes sugestões.

Ao Prof. Dr. Ian J. Thompson por ter me recebido na University of Surrey e por ter me ensinado a trabalhar com o código de canais acoplados FRESCO.

Aos meus colegas de departamento Fernando Medeiros, Julian Shorto, Dr. Davi Monteiro da Silva, Roberto Linares e Pedro Neto de Faria pelas produtivas discussões acerca das mais importantes questões.

Ao Dr. Franscisco de Assis Souza pelas valiosas dicas sobre a elaboração da tese em LTEX.

Aos colegas de graduação, em especial Marcus Cima Ferraz, Rafael Ribeiro Brandão, Aline de Almeida Vidotto, José Henrique Groh de C. Moura, Washington R. de Carvalho Jr. e Fernando 
Nascimento, que me ajudaram na caminhada até aqui, compartilhando muitos momentos alegres.

À FAPESP, pelo apoio financeiro.

Aos meus colegas de grupo, Prof. Dr. Dirceu Pereira, Dra. Cely de P. Silva, Dr. Ernesto S. Rossi Jr. e Deberson Pereira de Sousa, e aos ex-colegas de grupo Dr. Marcos Aurelio G. Alvarez e Dr. Leandro R. Gasques, pela sempre imediata prontidão em ajudar.

A todos os meus grandes amigos do Two Towers pela inestimável convivência e real Amizade ao longo de tantos anos.

Ao Dr. Celso Charuri pelo Exemplo e Eternos Ensinamentos. 


\section{Resumo}

Nesta tese de doutoramento foram propostos dois modelos teóricos (ZPM e GFA) para a obtenção da seção de choque de fusão e um (ZPM) para o cálculo do potencial de polarização para o canal elástico e correspondente seção de choque de espalhamento.

Foi usado em todos os cálculos deste trabalho, tanto no tratamento da fusão quanto no do espalhamento elástico, o Potencial de São Paulo (SP), que possui como uma das principais características a total ausência de parâmetros ajustáveis. Conseqüentemente, todos os cálculos apresentados no contexto de diferentes modelos constituem previsões teóricas ao invés de simples ajustes de dados.

Em uma análise comparativa acerca dos cálculos para a fusão, os modelos GFA e ZPM se mostraram equivalentes e compatíveis com cálculos de canais acoplados usuais, com a vantagem de incorporarem acoplamentos à toda banda vibracional e de permitirem a obtenção de cálculos acurados de seções de choque de fusão em energias extremamente abaixo da barreira. Aplicamos o modelo de ZPM ao cálculo da função de excitação de fusão para 112 sistemas de íons pesados, envolvendo tanto núcleos par-par como núcleos ímpar-par e ímpar-ímpar (incluindo alguns núcleos fracamente ligados), e confrontamos os resultados obtidos com dados experimentais existentes na literatura. Enquanto o modelo de penetração de barreira não-deformado prevê discordâncias de até onze ordens de magnitude, as previsões do modelo de ZPM mostraram-se de acordo com os dados dentro de duas ordens de grandeza.

Ao confrontarmos os resultados do modelo de ZPM para as seções de choque de espalhamento elástico com dados experimentais para alguns sistemas em energias em torno da barreira coulombiana, pudemos constatar uma boa concordância. Desta forma, demonstramos que é possível tratar os canais de espalhamento elástico e de fusão de maneira consistente dentro do mesmo contexto.

Palavras-chave: Fusão Nuclear, Espalhamento Elástico, Canais Acoplados, Modelos Teóricos. 


\section{Abstract}

In this Ph.D. thesis two theoretical models (ZPM and GFA) were proposed in order to obtain fusion cross sections and one (ZPM) to calculate the polarization potential for the elastic channel and the corresponding scattering cross section.

The São Paulo (SP) potential, which has as one of its main characteristics the complete absence of adjustable parameters, was used as bare interaction in the calculations of the present work. Therefore, all results, presented in the context of different models, correspond to theoretical predictions instead of simply data fit.

After a comparative analysis of fusion calculations, the GFA and ZPM models demonstrated to be equivalents and compatible with the usual coupled channel calculations, with the advantage of incorporating inelastic couplings to the complete vibrational band and of providing accurate fusion cross sections at extreme low energies relative to the Coulomb barrier. We applied the ZPM model to the calculation of the fusion excitation function for 112 heavy-ion systems, involving not only even-even nuclei but also odd-even and odd-odd ones (including some weakly-bound nuclei), and we compared the theoretical results with experimental data existing in literature. While the usual non-deformed barrier penetration model provides enhancements up to eleven orders of magnitude, the ZPM model predictions are in good agreement with the data within only two orders of magnitude.

When confronting the ZPM model results with experimental data of elastic scattering cross sections for some systems, at energies around the Coulomb barrier, we found out good agreement between them. Therefore, we demonstrated that it is possible to treat the elastic scattering and fusion channels in a consistent manner, within the same context.

Palavras-chave: Nuclear Fusion, Elastic Scattering, Coupled Channel, Theoretical Models. 


\section{Sumário}

$\begin{array}{ll}\text { Lista de Figuras } & \text { xv }\end{array}$

Lista de Tabelas $\quad$ xxiii

Trabalhos Publicados Pelo Autor $\quad$ xxv

Trabalhos Submetidos Pelo Autor para Publicação $\quad$ xxvii

1 Introdução 1

2 Modelos Unidimensionais $\quad 5$

2.1 Canais Acoplados . . . . . . . . . . . . . . . . . 6

2.2 Aproximação Congelada . . . . . . . . . . . . . . . . . . 10

2.3 FA Generalizada para Energia de Excitação Finita . . . . . . . . . . . . . . . 13

2.4 Modelo do Movimento de Ponto Zero . . . . . . . . . . . . . . . . . . . . 18

3 Potenciais e Deformações $\quad 25$

3.1 Sistemática de Densidades Nucleares . . . . . . . . . . . . . . . . 26

3.2 Sistemática de Comprimentos de Deformação . . . . . . . . . . . . . . . . 28

3.3 Potencial Coulombiano . . . . . . . . . . . . . . . . 32

3.3.1 Potencial Coulombiano Esférico . . . . . . . . . . . . . 33

3.3.2 Potencial Coulombiano Deformado . . . . . . . . . . . . . . . . 34

3.4 Potencial de São Paulo . . . . . . . . . . . . . . . . . . . . . . . . . . 40

3.4.1 Potencial de São Paulo Esférico . . . . . . . . . . . . . . . . . 40

3.4.2 Potencial de São Paulo Deformado . . . . . . . . . . . . . . . . . . 42 


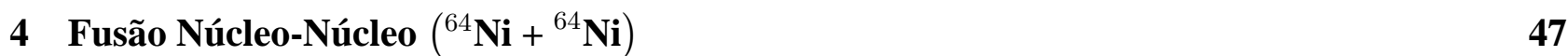

4.1 Potencial Efetivo e Fatores de Forma . . . . . . . . . . . . . . . . . . . . . . 48

4.2 Comparação entre os Modelos . . . . . . . . . . . . . . . . . . . 51

5 Sistemática de Fusão para Núcleos Par-Par $\quad 57$

5.1 Definição dos Parâmetros do Modelo . . . . . . . . . . . . . . . . . . 57

5.2 Comparação com os Dados Experimentais . . . . . . . . . . . . . . . 58

5.3 Discussão dos Resultados do Modelo de ZPM . . . . . . . . . . . . . . . . . . 67

6 Modelo para o Potencial de Polarização $\quad 75$

$7 \quad$ Fusão de Núcleos Ímpares $\quad 83$

7.1 Sistemática de Energias de Excitação . . . . . . . . . . . . . . . . . . . . . 84

7.2 Modelo de ZPM para Núcleos Ímpares . . . . . . . . . . . . . . . . . . . . 88

7.3 Comparação com Dados e Discussão dos Resultados . . . . . . . . . . . . . . . 88

8 Conclusão $\quad 97$

A Solução Numérica do Sistema de Equações Acopladas $\quad 101$

A.1 Resolvendo numericamente uma equação desacoplada . . . . . . . . . . . . . . 102

A.2 Tentativa de generalização para equações acopladas . . . . . . . . . . . . . . 103

A.3 Solução por inversão de matriz . . . . . . . . . . . . . . . . . . . . . 103

B Dedução Analítica das Projeções $U_{m n} \quad 107$

C Autofunção Analítica por Séries de uma Partícula através de uma Barreira Parabólica 111

D Aproximação WKB para uma Partícula através de uma Barreira Parabólica $\quad 115$

D.1 Primeiro Caso: $E \gg V_{B} \ldots \ldots \ldots \ldots \ldots \ldots$

D.2 Segundo Caso: $E \ll V_{B} ; E<V_{B}-V_{B 0} \ldots \ldots \ldots \ldots \ldots \ldots$

D.3 Terceiro Caso: $E \ll V_{B} ; E>V_{B}-V_{B 0} \ldots \ldots \ldots \ldots \ldots$

E Potenciais de Polarização para Excitações Coulombianas $\quad 123$

E.1 Desenvolvimento Teórico . . . . . . . . . . . . . . . . . . . . 123

Referências bibliográficas $\quad 132$ 


\section{Lista de Figuras}

2.1 Coeficientes de transmissão total, obtidos através de cálculos de CC, como funções da energia cinética para várias energias de excitação. A linha cheia representa os resultados obtidos sem considerar nenhum acoplamento. . . . . . . . . . . . .

2.2 Partes reais e imaginárias do potencial de polarização obtidas para diferentes valores de energia cinética e de excitação. As linhas cheias representam os resultados exatos de cálculos de CC enquanto as linhas tracejadas correspondem aos resultados do método descrito na Seção 2.4, o modelo proposto no presente trabalho e referido como Movimento de Ponto Zero. As linhas cheias e tracejadas se mostram praticamente indistinguíveis na figura. . . . . . . . . . . . . . . . .

2.3 Parte real de $V_{\text {PPDS }}$ (a) e a soma deste com $V(r, s)$ (linhas cheias) (b), obtido em diferentes condições. As linhas tracejadas em (b) correspondem somente à $V(r, s)$. Em um dos casos, a mudança na altura da barreira $\left(\Delta V_{B}\right)$ é indicada na figura. . . . . 14

2.4 Coeficientes de transmissão obtidos de cálculos de CC (linhas tracejadas) e modelo de FA generalizada (linhas cheias), assumindo que $\Delta V_{B}$ é representado pela Eq. (2.31) $(a, b)$ ou pela Eq. (2.32) (c). Os cálculos foram feitos para diversos valores de energia de excitação. Na figura, as linhas pontilhadas representam os resultados obtidos sem considerar qualquer acoplamento. . . . . . . . . . . . . .

2.5 A figura apresenta uma comparação entre os resultados para o coeficiente de transmissão total obtidos dos modelos de CC (Seção 2.1), GFA (Seção 2.3), Eq. (2.39) e somente potencial bare, para duas energias de excitação diferentes. Os resultados são apresentados escala linear (em cima) e logarítmica (embaixo) . . . . . . . . . . 
2.6 Coeficientes de reflexão do canal elástico $\left(R_{0}=\left|b_{0}\right|^{2}\right)$, em cima, e fase de $b_{0}$, embaixo, para duas energias de excitação. As linhas cheias representam os resultados exatos de CC. As linhas tracejadas correspondem aos resultados obtidos resolvendo a Eq. (2.15) desacoplada, com o potencial de polarização obtido de acordo com o método de ZPM. Como comparação, as linhas pontilhadas na figura representam os resultados sem considerar nenhum acoplamento. . . . . . . . . . . . . .

3.1 Valores equivalentes de difusividade $a$ obtidos para distribuições de carga extraídos de experimentos de espalhamento de elétrons e para densidades teóricas obtidas de cálculos de Dirac-Hartree-Bogoliubov. . . . . . . . . . . . . . . . . . . .

3.2 Valores do parâmetro de raio não deformado $R_{0}$ obtidos para distribuições de carga extraídos de experimentos de espalhamento de elétrons e para densidades teóricas obtidas de cálculos de Dirac-Hartree-Bogoliubov. . . . . . . . . . . . . . . . . . 28

3.3 Os valores dos comprimentos de deformação de quadrupolo (a) e de octupolo (b) como uma função do número de prótons (embaixo) ou nêutrons (em cima). As linhas tracejadas representam os números mágicos. . . . . . . . . . . . . . .

3.4 Os valores médios dos comprimentos de deformação de quadrupolo (a) e octupolo (b) para isótopos (pontos brancos) ou isótonos (pontos pretos) como função do número de prótons $Z$ (pontos brancos) ou de nêutrons $N$ (pontos pretos) do núcleo. . . . . . 3

3.5 As funções $D_{2}$ e $D_{3}$ que descrevem o comportamento médio dos comprimentos de deformação. As linhas tracejadas representam os números mágicos. . . . . . . . . . 30

3.6 Figura esquemática da colisão de dois núcleos deformados. A circunferência tracejada representa a superfície do núcleo 1 se não houvesse deformação. . . . . . . . . . .

3.7 O potencial coulombiano não-deformado para o sistema ${ }^{58} \mathrm{Ni}+{ }^{58} \mathrm{Ni}$ calculado através de diferentes modelos. A seta representa a posição aproximada do raio da barreira para a onda $s$ correspondente. . . . . . . . . . . . . . . . 35

3.8 A figura apresenta o potencial coulombiano total $\left(V_{C}\right)$, a correção $\left(V_{C}^{\text {Cor }}\right)$ e os valores relativos correspondentes $\left(100 V_{C}^{\mathrm{Cor}} / V_{C}\right)$ para o sistema ${ }^{58} \mathrm{Ni}+{ }^{58} \mathrm{Ni}$. A seta indica a posição aproximada do raio da barreira. . . . . . . . . . . . . . . . . 36 
3.9 A figura apresenta a correção do potencial coulombiano para o sistema ${ }^{58} \mathrm{Ni}+{ }^{58} \mathrm{Ni}$. O núcleo 2 é assumido como não-deformado e a difusividade de ambas distribuições é $a=0$. Os parâmetros de deformação do núcleo são indicados na figura. Os "pontos experimentais" na figura correspondem, na verdade, a simulações de Monte Carlo para a resolução da integral em seis dimensões representada pela Eq. (3.14). As linhas cheias representam os resultados das Eqs. (3.20) - (3.27) e as linhas tracejadas representam a expressão assintótica (3.28). As setas indicam a posição aproximada do raio da barreira. . . . . . . . . . . . . . . . . . . .

3.10 As figuras apresentam as correções do potencial coulombiano para o sistema ${ }^{58} \mathrm{Ni}+$ ${ }^{58} \mathrm{Ni}$, onde $\beta_{2}=0.205, \beta_{3}=0.235$. Os ângulos dos eixos de deformação são indicados nas figuras. Os "pontos experimentais" na figura correspondem, na verdade, a simulações de Monte Carlo para a resolução da integral em seis dimensões representada pela Eq. (3.14). As linhas cheias representam os resultados das Eqs. (3.29) - (3.32) e as linhas tracejadas representam a expressão assintótica (3.28). As setas indicam a posição aproximada do raio da barreira. . . . . . . . . . . . . . . .

3.11 O potencial de São Paulo nas abordagens de alcance zero (linhas tracejadas) e finito (linhas cheias) para quatro sistemas indicados na figura. . . . . . . . . . . .

3.12 O potencial nuclear deformado (linhas cheias) é comparado com o não-deformado (linhas tracejadas) para o sistema ${ }^{58} \mathrm{Ni}+{ }^{58} \mathrm{Ni}$. A figura apresenta também os resultados obtidos com as aproximações usuais, Eqs. (3.40) e (3.41). $\beta_{2}=0.205, \beta_{3}=0.235$ e $a_{M}=0.56 \mathrm{fm}$ foram assumidos para ambos os núcleos. Os ângulos dos eixos de deformação são indicados na figura. . . . . . . . . . . . . . . . .

$3.13 \mathrm{O}$ valor absoluto da correção $V_{F}^{C o r}=V_{F}-V_{F}^{(0)}$, onde o potencial de convolução foi obtido a partir das Eqs. (3.37), (3.40) e (3.41). Os parâmetros de deformação são os mesmos daqueles da Figura $3.12 \ldots \ldots \ldots \ldots \ldots$

4.1 Dados experimentais e correspondentes funções de excitação de fusão para o sistema ${ }^{64} \mathrm{Ni}+{ }^{64} \mathrm{Ni}$. As linhas correspondem a diferentes abordagens teóricas para o cálculo das seções de choque teóricas (ver texto para detalhes). A posição da altura da barreira para a onda $s$ do potencial não deformado $\left(V_{B 0}\right)$ é mostrada na figura. Existem diferentes conjuntos de dados (representados na figura por diferentes símbolos) obtidos em diferentes trabalhos, com seções de choque que diferem entre si por um fator em torno de 3 para algumas energias. . . . . . . . . . . . . . . . 
4.2 Dados experimentais e correspondentes seções de choque teóricas de BPM não-deformada (linha tracejada) e de ZPM considerando as bandas completas (linha cheia) para o sistema ${ }^{64} \mathrm{Ni}+{ }^{64} \mathrm{Ni}$. As outras linhas indicam cálculo de $\mathrm{CC}$ com o código FRESCO e com o modelo representado pelas Eqs. (2.43) e (2.44), onde apenas os acoplamentos aos estados $2^{+}$e $3^{-}$foram considerados. Existem diferentes conjuntos de dados (representados na figura por diferentes símbolos) obtidos em diferentes trabalhos, com seções de choque que diferem entre si por um fator em torno de 3 para algumas energias. . . . . . . . . . . . . . . . .

4.3 Seções de choque parcial de fusão em quatro energias, obtidas de cálculos de CC com o código Fresco para o sistema ${ }^{64} \mathrm{Ni}+{ }^{64} \mathrm{Ni}$. . . . . . . . . . . . . . . . .

5.1 Dados experimentais de fusão e correspondentes seções de choque teóricas para BPM não-deformado (linhas tracejadas) e para o modelo de ZPM (linhas cheias) para os sistemas ${ }^{12} \mathrm{C}+{ }^{12} \mathrm{C} \mathrm{e}{ }^{16} \mathrm{O}+{ }^{16} \mathrm{O}$. As linhas pontilhadas representam os resultados de FA, i.e. aqueles obtidos considerando energias de excitação nulas. As alturas das barreiras dos potenciais não-deformados para as ondas $s$ são apresentadas na figura. .

5.2 Dados de fusão e correspondentes seções de choque teóricas para BPM não-deformado (linhas tracejadas) e para o modelo de ZPM (linhas cheias) para os sistemas indicados nas figuras. As alturas das barreiras dos potenciais não-deformados para as ondas $s$ são apresentadas na figura.

5.3 Dados de fusão e correspondentes seções de choque teóricas para BPM não-deformado (linhas tracejadas) e para o modelo de ZPM (linhas cheias) para os sistemas indicados nas figuras. As alturas das barreiras dos potenciais não-deformados para as ondas $s$ são apresentadas na figura.

5.4 Seção de choque total de fusão (círculos fechados) para o sistema ${ }^{40} \mathrm{Ar}+{ }^{144} \mathrm{Sm}$. As contribuições de resíduo de evaporação (círculos abertos) e de fusão-fissão (círculos semi-abertos) para a fusão total também são apresentadas. As linhas tracejadas e cheias correspondem às seções de choque de BPM e ZPM, respectivamente. . . . . . .

5.5 Seções de choque de resíduo de evaporação para diversos sistemas envolvendo ${ }^{86} \mathrm{Kr}$ e ${ }^{90} \mathrm{Zr}$ como projétil. As linhas tracejadas e cheias correspondem às seções de choque de BPM e ZPM, respectivamente. . . . . . . . . . . . . . . . . . . 
5.6 Dados de fusão e correspondentes seções de choque teóricas para BPM não-deformado (linhas tracejadas) e para o modelo de ZPM (linhas cheias) para os sistemas indicados nas figuras. As alturas das barreiras dos potenciais não-deformados para as ondas $s$ são apresentadas na figura. No caso dos sistemas ${ }^{58} \mathrm{Ni}+{ }^{112,124} \mathrm{Sn}$, incertezas experimentais de $1 \mathrm{MeV}$ nos correspondentes valores de energia são indicados na figura. . .

5.7 Dados de seção de choque de fusão (embaixo) e correspondentes distribuições de barreira (em cima) para os sistemas ${ }^{16} \mathrm{O}+{ }^{238} \mathrm{U},{ }^{28} \mathrm{Si}+{ }^{208} \mathrm{~Pb}$ e ${ }^{32,36} \mathrm{~S}+{ }^{110} \mathrm{Pd}$. As linhas tracejadas e cheias correspondem às seções de choque de BPM e ZPM, respec-

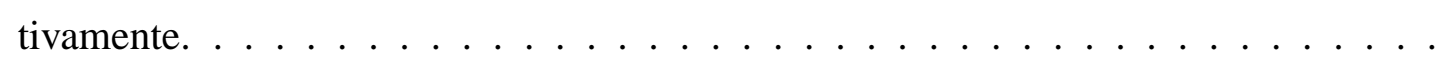

5.8 Razões entre dados de fusão e seções de choque teóricas de BPM (lado esquerdo) e entre os dados e cálculos do modelo de ZPM (lado direito) para os sistemas apresentados nas Figuras 5.1 a 5.7 e na Figura 4.2 (página 55) . . . . . . . . . . . . .

5.9 Dados de seção de choque de fusão para os sistemas ${ }^{40} \mathrm{Ca},{ }^{40} \mathrm{Ar}+{ }^{116} \mathrm{Sn}$. As linhas tracejadas e cheias correspondem às seções de choque de BPM e ZPM (padrão), respectivamente. As outras linhas correspondem a cálculos de ZPM onde os valores de $\beta$ ou da difusividade das densidades $a$ foram modificados (ver texto para detalhes). .

5.10 Os símbolos representam densidades de matéria teóricas calculadas através do modelo de Dirac-Hartree-Bogoliubov. As linhas cheias e tracejadas correspondem a distribuições $2 \mathrm{pF}$ com diferentes valores de difusividades. Os raios das distribuições $2 \mathrm{pF}$ foram obtidos pela Eq. (3.6). . . . . . . . . . . . . . .

5.11 Valores médios das ordens de magnitude dos desvios entre os dados de fusão e as seções de choque de ZPM (ver texto para detalhes) . . . . . . . . . . . . . . .

6.1 EMBAIXO: Potencial efetivo para a onda $s$ para potenciais não-deformado (linha cheia) e deformado (linha tracejada) para o sistema ${ }^{58} \mathrm{Ni}+{ }^{64} \mathrm{Ni}$. A deformação corresponde apenas ao modo $\lambda=2$ do ${ }^{58} \mathrm{Ni}$, com $\beta_{21}=0.215$. As linhas pontilhadas representam barreiras parabólicas que resultam em uma mesma altura e curvatura de barreira do potencial efetivo. As setas indicam os correspondentes raios de barreira. A variação da altura da barreira também é indicada na figura. EM CIMA: Intensidade de acoplamento no caso de $\lambda=2$ e $i=1\left({ }^{58} \mathrm{Ni}\right)$ como calculado pela Eq. (6.1). A seta na figura indica o ponto $R_{\text {corte }}$ onde $F_{21}=0 \ldots \ldots \ldots$ 
6.2 Distribuições angulares de espalhamento elástico para o sistema ${ }^{58} \mathrm{Ni}+{ }^{64} \mathrm{Ni}$ nas energias $E_{c . m} .=95.6,99.5,106.5$ e $115 \mathrm{MeV}$. As linhas correspondem a diferentes cálculos teóricos (ver texto para detalhes). . . . . . . . . . . . . . .

6.3 Módulo da matriz S para as quatro distribuições angulares de espalhamento elástico analisadas no presente capítulo. . . . . . . . . . . . . . . .

6.4 Partes real e imaginária do potencial de polarização para a onda parcial grazing $L=\ell_{g}$ para as quatro distribuições angulares analisadas na Figura 6.2. As linhas cheias e tracejadas na figura representam os resultados de cálculos de ZPM e de CC respectivamente. Foram considerados nos cálculos de CC os acoplamentos nuclear e coulombiano aos estados $2^{+}$e $3^{-}$de ambos os núcleos. As setas na figura representam a posição do raio da barreira da onda $\ell_{g}$ do potencial não-deformado. As linhas pontilhadas marcam o nível $V_{\mathrm{pol}}=0$.

6.5 Partes real e imaginária do potencial de polarização para $\ell=30$ e 60 na energia de $E_{c . m .}=106.5 \mathrm{MeV}$. As linhas pontilhadas marcam o nível $V_{\mathrm{pol}}=0 . \ldots \ldots$. . .

6.6 Distribuições angulares de espalhamento elástico para o sistema ${ }^{16} \mathrm{O}+{ }^{58} \mathrm{Ni}$ em diversas energias. As linhas tracejadas correspondem aos cálculos de BPM e as linhas cheias representam as previsões teóricas de ZPM. . . . . . . . . . . . . . . . .

6.7 Distribuições angulares de espalhamento elástico para o sistema ${ }^{16} \mathrm{O}+{ }^{208} \mathrm{~Pb}$ em diversas energias. As linhas tracejadas correspondem aos cálculos de BPM e as linhas cheias representam as previsões teóricas de ZPM.

7.1 Valores experimentais das energias de excitação dos estados $2^{+}$e $3^{-}$como uma função do número de prótons (embaixo) ou nêutrons (em cima) dos núcleos. As linhas pontilhadas representam os números mágicos.

7.2 Valores médios para isótopos (ou isótonos) das energias de excitação dos estados $2^{+}$ e $3^{-}$como uma função do número de prótons (ou nêutrons) dos núcleos. . . . . . . . .

7.3 As funções $E_{2}$ e $E_{3}$ que descrevem aproximadamente o comportamento das energias de excitação. As linhas pontilhadas representam os números mágicos. . . . . . . . .

7.4 Dados de fusão e correspondentes seções de choque teóricas de BPM não-deformada (linhas tracejadas) e de ZPM (linhas cheias) para os sistemas indicados nas figuras. As alturas da barreira da onda s do potencial não-deformado são apresentadas nas figuras. 
7.5 Dados de fusão e correspondentes seções de choque teóricas de BPM não-deformada (linhas tracejadas) e de ZPM (linhas cheias) para os sistemas indicados nas figuras. As alturas da barreira da onda s do potencial não-deformado são apresentadas nas figuras. Em alguns casos, existem diferentes conjuntos de dados (indicados por diferentes símbolos) com seções de choque medidas que diferem por até uma ordem de magnitude. . . . . . . . . . . . . . . . . . . . .

7.6 Razões entre dados de fusão e seções de choque teóricas de BPM (lado esquerdo) ou ZPM (lado direito) para sistemas envolvendo núcleos ímpares (painéis de baixo) ou apenas par-par (painéis de cima) . . . . . . . . . . . . . . .

7.7 Valores médios da ordem de magnitude dos desvios entre dados de fusão e seções de choque de ZPM para sistemas envolvendo núcleos ímpares e/ou par-par e também

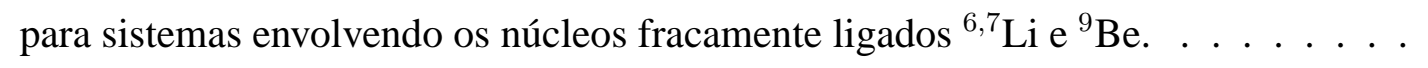

7.8 Dados de fusão e correspondentes seções de choque teóricas de BPM não-deformada (linhas tracejadas) e de ZPM (linhas cheias) para os sistemas indicados nas figuras. As alturas da barreira da onda s do potencial não-deformado são apresentadas nas figuras. 


\section{Lista de Tabelas}

3.1 Valores da função $D_{2}(X) \ldots \ldots \ldots \ldots \ldots \ldots \ldots \ldots \ldots \ldots \ldots \ldots \ldots \ldots \ldots$

5.1 A tabela apresenta os valores dos parâmetros de deformação de quadrupolo $\left(\beta_{2}\right)$ e de octupolo $\left(\beta_{3}\right)$ e também as correspondentes energias de excitação para os núcleos estudados nesta sistemática. . . . . . . . . . . . . . . . . . . . . . 59

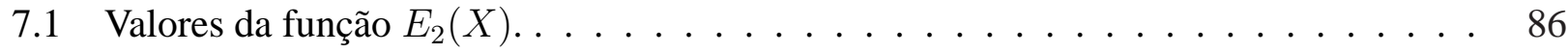

7.2 Valores da função $E_{3}(X) \ldots \ldots \ldots \ldots \ldots$. . . . . . . . . . . 87

7.3 A tabela apresenta os valores dos parâmetros de deformação de quadrupolo $\left(\beta_{2}\right)$ e de octupolo $\left(\beta_{3}\right)$, e também das correspondentes energias de excitação para os núcleos estudados neste capítulo. . . . . . . . . . . . . . . . . . . . 89

E.1 Os fatores semi-clássicos de perda de energia $g_{\lambda}(\xi)$ para excitações de quadrupolo e octupolo. 


\section{Trabalhos Publicados Pelo Autor}

1. Consistent analysis of fusion data without adjustable parameters for a wide variety of heavy-ion systems - G. P. A. Nobre, L. C. Chamon, L. R. Gasques, B. V. Carlson, I. J. Thompson; Physical Review C vol. 75, 044606 (2007)

2. Tunneling through a parabolic barrier coupled to an oscillatory degree of freedom: application to heavy-ion fusion at sub-barrier energies - G. P. A. Nobre, L. C. Chamon, B. V. Carlson, I. J. Thompson, L. R. Gasques; Nuclear Physics A vol. 786, 90-106 (2007)

3. ${ }^{18} \mathrm{O}+{ }^{110} \mathrm{Pd}$ : Measurements and realistic coupled-channel analysis in a transitional region - D. Pereira, E. S. Rossi, Jr., G. P. A. Nobre, L. C. Chamon, C. P. Silva, L. R. Gasques, M. A. G. Alvarez, R. V. Ribas, J. R. B. Oliveira, N. H. Medina, M. N. Rao, E. W. Cybulska, W. A. Seale, N. Carlin, P. R. S. Gomes, J. Lubian, R. M. Anjos; Physical Review C vol. 74, 034608 (2006)

4. Coulomb and nuclear potentials between deformed nuclei applied to the fusion process - L. C. Chamon, G. P. A. Nobre, D. Pereira, C. P. Silva, E. S. Rossi, Jr., L. R. Gasques, B. V. Carlson; Brazilian Journal of Physics vol. 35 no.3b 906-908 (2005)

5. Elastic, inelastic scatterings and transfer reactions for ${ }^{16,18} \mathrm{O}$ on ${ }^{58} \mathrm{Ni}$ described by the São Paulo potential - J. J. S. Alves, P. R. S. Gomes, J. Lubian, L. C. Chamon, R. M. Anjos, D. Pereira, E. S. Rossi, Jr., C. P. Silva, M. A. G. Alvarez, G. P. A. Nobre, L. R. Gasques; Brazilian Journal of Physics vol. 35 no.3b 909-911 (2005)

6. Consistent analysis of peripheral reaction channels and fusion for the ${ }^{16,18} \mathbf{O}+{ }^{58} \mathbf{N i}$ systems $-\mathrm{J}$. J. S. Alves, P. R. S. Gomes, J. Lubian, L. C. Chamon, D. Pereira, R. M. Anjos, E. S. Rossi, Jr., C. P. Silva, M. A. G. Alvarez, G. P. A. Nobre, L. R. Gasques; Nuclear Physics A, vol. 748, 59-74, (2005)

7. Coulomb and nuclear potentials between deformed nuclei - L. C. Chamon, G. P. A. Nobre, D. Pereira, E. S. Rossi, Jr., C. P. Silva, L. R. Gasques, B. V. Carlson; Physical Review C vol. 70, 014604 (2004)

8. Systematical study of the optical potential for systems like $\mathrm{A}+{ }^{58} \mathrm{Ni}$ from sub-barrier data analyses - L. R. Gasques, L. C. Chamon, D. Pereira, M. A. G. Alvarez, E. S. Rossi, Jr., C. P. Silva, G. P. A. Nobre, B. V. Carlson; Physical Review C vol. 67, 067603 (2003) 


\section{Trabalhos do Autor Submetidos para Publicação}

1. Consistent analysis of fusion data without adjustable parameters for systems involving odd nuclei - G. P. A. Nobre, C. P. Silva, L. C. Chamon, B. V. Carlson; Submetido para publicação na Physical Review $\mathbf{C}$

2. Polarization potential for the heavy-ion elastic scattering process - G. P. A. Nobre, L. C. Chamon, B. V. Carlson; Submetido para publicação na Nuclear Physics A 
Xxviii 


\section{Capítulo 1}

\section{Introdução}

O processo de fusão de íons pesados tem sido extensivamente estudado durante as últimas décadas [1], e uma grande quantidade de dados experimentais já foi obtida para uma grande variedade de sistemas. Mesmo assim, muitas questões neste campo não estão ainda completamente solucionadas. É bem conhecido que dados de seção de choque de fusão para sistemas de íons pesados mostram grandes enhancements ${ }^{1}$ em energias abaixo da barreira em comparação com previsões teóricas do Modelo de Penetração de Barreira $\left(\mathrm{BPM}^{2}\right)$ [2]. Em diversos trabalhos (e.g. [3, 4]), os enhancements puderam ser explicados para diversos particulares sistemas considerando a estrutura interna dos núcleos participantes através de cálculos de canais acoplados ( $\mathrm{CC}$, do inglês Coupled-Channels). Análises de dados pelo método de CC e a descoberta da conexão entre derivadas em energia da seção de choque e distribuições de barreira [5] motivaram a obtenção de dados de alta precisão. Em trabalhos recentes (ver e.g. Refs. [6-10]) a fusão de íons pesados em energias extremamente abaixo da barreira é discutida. Em alguns desses trabalhos, foi observado que os valores de difusividade para o potencial ajustado para reproduzir os dados de fusão são significativamente maiores do que aqueles usualmente assumidos para análises de dados de espalhamento elástico. Conseqüentemente, a consistência das análises de dados de fusão e de espalhamento elástico é um objetivo a ser atingido.

Apesar de o formalismo de CC ser apropriado, do ponto de vista teórico, para descrever a fusão

\footnotetext{
${ }^{1} \mathrm{O}$ termo em inglês enhancement é comumente empregado em Física Nuclear para denotar um aumento da seção de choque experimental em relação ao cálculo usual de penetração de barreira não-deformada.

${ }^{2}$ A sigla BPM, do inglês Barrier Penetration Model, é bastante usada ao se referir ao Modelo de Penetração de Barreira não-deformada.
} 
de íons pesados, problemas numéricos podem ocorrer na solução das equações de CC, resultando em seções de choque teóricas não confiáveis, principalmente em energias muito abaixo da barreira, quando um grande número de canais é incluído nos cálculos. Este é um assunto importante visto que, por exemplo, análises de dados recentes [11] demonstraram a sensibilidade a excitações de multi-fônons em reações de fusão para íons pesados. Por outro lado, muitas tentativas de melhorar o BPM foram feitas considerando deformações permanentes e/ou vibracionais dos núcleos (e.g. [12]). Para excitações inelásticas, considerando a Aproximação Congelada (FA, do inglês Frozen Approximation), o coeficiente de transmissão pode ser obtido efetuando-se uma média sobre diferentes configurações do sistema. Para energias de excitação desprezíveis, este procedimento é equivalente ao cálculo de $\mathrm{CC}$, onde a base completa de estados é considerada. O efeito da energia de excitação finita já foi estudado em diversos trabalhos (ver e.g. [13-16]). No presente trabalho, propomos um modelo para o cálculo de seções de choque de fusão que chamamos de Aproximação Congelada Generalizada (GFA, do inglês Generalized Frozen Approximation) que, como o próprio nome sugere, generaliza o método de FA para o caso de energia de excitação não nula. Também neste trabalho, nós generalizamos os resultados obtidos na Ref. [14] com o objetivo de efetuar cálculos de fusão para íons pesados. No contexto deste outro modelo, que denominamos Movimento de Ponto Zero (ZPM, do inglês Zero Point Motion), nós consideramos o efeito dos acoplamentos dos conjuntos completos de estados inelásticos conectados com as bandas vibracionais associadas aos estados $2^{+}$e $3^{-}$de núcleos par-par, levando em consideração as energias de excitação. Apesar do grande número de canais acoplados, a convergência numérica de nossos cálculos é bastante boa, o que constitui uma vantagem em relação ao cálculo de CC usual. Além da fusão, também aplicamos o modelo de ZPM para o cálculo do potencial de polarização e de seções de choque de espalhamento elástico, obtendo resultados compatíveis com os dados experimentais.

Na maioria dos trabalhos que lidam com análises de dados de fusão, alguns poucos parâmetros ajustáveis relacionados com o potencial nuclear e/ou com amplitudes de acoplamento foram usados de maneira a descrever os dados experimentais. Apesar do bom ajuste de dados geralmente obtido, este procedimento pode resultar em valores não realistas para os parâmetros livres que poderiam "mascarar" alguma característica particular do sistema. No presente trabalho, analisamos os dados de fusão para mais de cem diferentes sistemas de íons pesados sem usar qualquer parâmetro ajustável. 
As análises foram realizadas no contexto do modelo de ZPM e, em todo o trabalho apresentado nesta tese, foi usado o potencial de São Paulo (SP) [17-19] como interação nuclear. Uma importante característica do potencial de SP é a ausência de parâmetros livres. Desta forma, todos os resultados teóricos obtidos neste trabalho se tratam de previsões, e não meramente ajustes de dados. Neste contexto, características particulares no comportamento dos dados para diferentes sistemas podem ser claramente detectadas. O potencial de SP demonstrou ter sucesso em descrever o espalhamento elástico e canais periféricos de reação para diversos sistemas em uma ampla região de energia, desde energias abaixo da barreira até intermediárias [18, 20-35]. Logo, a interação nuclear aqui assumida para analisar os dados de fusão é, consistentemente, também apropriada para descrever o processo de espalhamento elástico. Em energias extremamente abaixo da barreira (em torno de $20 \mathrm{MeV}$ abaixo da barreira), o BPM subestima os dados por até onze ordens de magnitude enquanto os resultados do modelo de ZPM concordam com os dados dentro de apenas duas ordens de grandeza para a maioria dos sistemas. Nós mostramos que as previsões podem ser significativamente melhoradas com o auxílio de parâmetros ajustáveis, mas, neste caso, os valores obtidos para estes parâmetros podem se tornar não realistas.

No Capítulo 2 descrevemos, para melhor compreensão, versões unidimensionais dos modelos $\operatorname{citados}^{3}$ : CC, FA, GFA e ZPM, sendo estes dois últimos por nós desenvolvidos. Os quatro modelos são apresentados considerando uma barreira parabólica acoplada linearmente a um grau de liberdade interno de oscilador harmônico. Dedicamos o Capítulo 3 à descrição dos potenciais usados nos cálculos apresentados nos capítulos subseqüentes. Apresentamos então, no Capítulo 4, uma comparação entre os modelos citados quando aplicados ao cálculo da função de excitação de fusão para o sistema ${ }^{64} \mathrm{Ni}+{ }^{64} \mathrm{Ni}$. Discutimos as qualidades e limitações de cada um, inclusive o problema do cálculo de seções de choque de fusão pelo método de CC em energias extremamente abaixo da barreira. Em seguida, no Capítulo 5, apresentamos uma sistemática que fizemos do cálculo de seções de choque de fusão através do modelo de ZPM que propusemos, aplicado a 64 sistemas envolvendo núcleos parpar. No Capítulo 6, apresentamos um método que desenvolvemos para estender o modelo de ZPM para o cálculo do potencial de polarização e, conseqüentemente, da seção de choque de espalhamento elástico. Aplicamos este método aos sistemas ${ }^{58} \mathrm{Ni}+{ }^{64} \mathrm{Ni},{ }^{16} \mathrm{O}+{ }^{58} \mathrm{Ni}$ e ${ }^{16} \mathrm{O}+{ }^{208} \mathrm{~Pb}$ e apresentamos

\footnotetext{
${ }^{3} \mathrm{Na}$ presente tese, os modelos estudados serão referidos com o auxílio das siglas de seus respectivos nomes em inglês, visto que são termos amplamente conhecidos e publicados na literatura
} 
os resultados obtidos. A seguir, no Capítulo 7, após apresentar uma sistemática das energias de excitação dos estados $2^{+}$e $3^{-}$dos núcleos par-par, fizemos uma interpolação para encontrar quais seriam os valores de energia de excitação dos estados equivalentes de "quadrupolo" e de "octupolo" para núcleos ímpares. Com base nesses resultados, e na sistemática de parâmetros de deformação apresentada no Capítulo 3, aplicamos o modelo de ZPM no cálculo de seções de choque de fusão para 48 sistemas envolvendo um ou dois núcleos ímpares (par-ímpar e ímpar-ímpar). Finalmente, no Capítulo 8, além de discussões e considerações finais, apresentamos um resumo das principais conclusões que puderam ser obtidas.

No período de desenvolvimento do trabalho correspondente a esta tese de doutoramento, o autor participou da elaboração de dez artigos, sendo dois submetidos para publicação e oito já publicados, dentre estes últimos, três na Physical Review C, três na Nuclear Physics A e dois na Brazilian Journal of Physics. Na Ref. [36], é descrito o modelo de ZPM e é mostrada a sistemática de suas previsões para a fusão para diversos sistemas envolvendo núcleos par-par, enquanto na Ref. [37] o modelo GFA é apresentado e aplicado aos sistemas ${ }^{58,64} \mathrm{Ni}+{ }^{58,64} \mathrm{Ni}$. Apresentamos nossa sistemática para parâmetros de deformação de quadrupolo e octupolo e as expressões que desenvolvemos para o cálculo do potencial coulombiano e do potencial de São Paulo para distribuições deformadas da matéria nuclear na Ref. [38]. As Refs. [20, 29, 33, 39, 40] apresentam trabalhos do qual o autor tomou parte, colaborando de alguma maneira, mas que não tratam de assuntos diretamente ligados à presente tese. Além disso, há um artigo [41] recentemente submetido à Nuclear Physics $\mathbf{A}$ tratando da aplicação do modelo de ZPM para o cálculo do potencial de polarização e do espalhamento elástico, e outro [42] tratando do modelo de ZPM para a fusão em sistemas envolvendo núcleos ímpares, recentemente submetido à Physical Review $\mathbf{C}$. 


\section{Capítulo 2}

\section{Modelos Unidimensionais}

Através da expansão da onda plana, que representa o feixe incidente em colisões nucleares, em ondas parciais de momento angular bem definido, podemos decompor o espalhamento tridimensional em espalhamentos em uma única dimensão para cada valor de momento angular. Desta forma, o estudo de modelos unidimensionais se mostra extremamente útil em problemas de espalhamento nuclear, visto que são muito mais simples de serem tratados, conceitual e numericamente, e podem facilmente ser generalizados para o caso real.

Neste capítulo serão descritos modelos unidimensionais tanto para o cálculo da probabilidade de transmissão por uma barreira, relacionado à fusão, bem como para o coeficiente de reflexão, relacionado, por sua vez, com os espalhamentos elástico e inelástico. Dasso, Landowne e Winter [14] já haviam estudado anteriormente um problema semelhante ao que propomos resolver aqui e obtiveram o coeficiente de transmissão no caso de acoplamento linear à uma barreira parabólica com um único grau de liberdade interno. Nós generalizamos os estudos da Ref. [14] para obter a transmissão no caso de vários graus de liberdade, que é importante para o estudo da fusão entre íons pesados. O modelo generalizado, que denominamos Movimento de Ponto Zero (ZPM, do inglês Zero Point Motion), permite obter também o potencial de polarização e, conseqüentemente, seções de choque de espalhamento elástico. A descrição deste modelo encontra-se na Seção 2.4. Também neste capítulo, na Seção 2.3, propomos um outro modelo alternativo para o cálculo dos coeficientes de transmissão, que denominamos Aproximação Congelada Generalizada (GFA, do inglês Generalized Frozen Approximation), que consiste, como o próprio nome sugere, de uma generalização para energias de excitação 
não-nulas da chamada Aproximação Congelada (FA, do inglês Frozen Approximation), também conhecida como Aproximação Súbita, descrita na Seção 2.2

\subsection{Canais Acoplados}

O método de canais acoplados (CC, do inglês Coupled-Channels) constitui, do ponto de vista teórico, o modelo mais imediato e fundamental pois o sistema de equações diferenciais acopladas, que dá nome ao modelo, surge naturalmente da equação de Schrödinger total do sistema após a expansão da função de onda total na base de auto-estados internos.

Consideremos o espalhamento de um núcleo por outro de forma que a massa reduzida do sistema seja $\mu$ e que a energia cinética assintótica da partícula incidente, no centro de massa, seja $E$. Consideremos também, a título de simplificação, que somente um dos núcleos (por exemplo, o alvo) possui estrutura interna, descrita por uma Hamiltoniana interna, com níveis de energia discretos que podem ser excitados durante o espalhamento. A cada um desses níveis está associado um canal de espalhamento inelástico. De forma a tornar o problema mais concreto, assumiremos que o mecanismo de excitação interna corresponde a um oscilador harmônico simples, associado à coordenada vibracional $s$, e que o espalhamento (coordenada $r$ ) se dá por uma barreira parabólica de potencial cuja altura está linearmente acoplada ao oscilador harmônico. A correspondente equação de Schrödinger pode então ser escrita, supondo que o alvo esteja inicialmente em seu estado fundamental, como:

$$
\begin{gathered}
{\left[H_{0}+H_{o s c}\right] \Psi(r, s)=\left(E+E^{*} / 2\right) \Psi(r, s)} \\
H_{0}(r, s)=-\frac{\hbar^{2}}{2 \mu} \frac{\partial^{2}}{\partial r^{2}}+V(r, s) \\
H_{o s c}(s)=-E^{*}\left[\sigma^{2} \frac{\partial^{2}}{\partial s^{2}}-\frac{s^{2}}{4 \sigma^{2}}\right]
\end{gathered}
$$

onde $H_{0}$ é a Hamiltoniana de espalhamento e $H_{o s c}$ é a Hamiltoniana de estrutura interna do alvo, descrita como um oscilador harmônico simples em $s$. A energia de excitação, $E^{*}$, e o parâmetro de massa do oscilador, $D$, se relacionam com o desvio padrão de vibração no estado fundamental, $\sigma$, 
através de $D=\hbar^{2} / 2 \sigma^{2} E^{*}$. A barreira parabólica é escrita como:

$$
\begin{gathered}
V(r, s)=V_{B}-F s-\frac{1}{2} \mu \omega^{2} r^{2},|r| \leq r_{0} \\
V(r, s)=0,|r|>r_{0}
\end{gathered}
$$

sendo que $r_{0}$ é definido como $\frac{1}{2} \mu \omega^{2} r_{0}^{2}=V_{B}$. Logo, nas regiões $r<-r_{0}$ e $r>r_{0}$ a partícula executa movimento livre. Como fica evidente pela Eq. (2.4), supomos que a altura da barreira parabólica é acoplada ao grau de liberdade do oscilador harmônico enquanto a curvatura da barreira, $\hbar \omega$, não é afetada pelos acoplamentos. Consideramos apenas, no presente desenvolvimento, um acoplamento linear, que é relacionado ao termo $F s$ na Eq. (2.4).

As funções de onda do oscilador são auto-vetores da Hamiltoniana interna de oscilador harmônico, $H_{o s c}$ na Eq. (2.3), com auto-valores de energia $(n+1 / 2) E^{*}$ para um dado auto-estado $|n\rangle$, e têm como forma explícita na representação de Schrödinger:

$$
|n\rangle=\frac{1}{\left(2 \pi \sigma^{2}\right)^{1 / 4}} e^{-s^{2} / 4 \sigma^{2}} \frac{H_{n}(s / \sigma \sqrt{2})}{\sqrt{n ! 2^{n}}}
$$

onde $H_{n}$ são os polinômios de Hermite. Expandindo a função de onda total na base das auto-funções de oscilador harmônico, $\Psi(r, s)=\sum \psi_{n}(r)|n\rangle$, e substituindo esta expansão na Eq. (2.1) obtém-se um sistema de equações diferenciais acopladas dependente apenas da coordenada $r$, cuja equação para o canal $n$ é escrita, na região $-r_{0} \leq r \leq r_{0}$, como:

$$
-\frac{\hbar^{2}}{2 \mu} \frac{d^{2} \psi_{n}(r)}{d r^{2}}+\left[V_{B}-\frac{1}{2} \mu \omega^{2} r^{2}\right] \psi_{n}(r)+\sum_{m \neq n} V_{n m} \psi_{m}(r)=\left[E-n E^{*}\right] \psi_{n}(r)
$$

onde

$$
V_{n m}=-F \sigma\left[\sqrt{n} \delta_{n, m+1}+\sqrt{m} \delta_{n, m-1}\right]
$$

Vemos, através da Eq. (2.7), que cada projeção $\psi_{n}(r)$ da base do oscilador harmônico, ou seja, a função de onda de cada canal, depende da solução para a função de onda de todos os outros canais, surgindo assim o acoplamento entre os canais. 
As funções de onda assintóticas devem se comportar como ondas livres:

$$
\begin{gathered}
\psi_{n}\left(r \geq r_{0}\right)=\delta_{n, 0} e^{-i k_{0} r}+b_{n} e^{i k_{n} r} \\
\psi_{n}\left(r \leq-r_{0}\right)=a_{n} e^{-i k_{n} r}
\end{gathered}
$$

sendo que os coeficientes de transmissão e reflexão totais são dados, respectivamente, por:

$$
\begin{aligned}
& T=\sum_{n} T_{n}=\sum_{n} \frac{k_{n}}{k_{0}}\left|a_{n}\right|^{2}, \\
& R=\sum_{n} R_{n}=\sum_{n} \frac{k_{n}}{k_{0}}\left|b_{n}\right|^{2},
\end{aligned}
$$

onde, obviamente, por conservação total de fluxo, obtém-se $T+R=1$.

No Apêndice A encontra-se descrito um método para resolver numericamente o sistema de canais acoplados correspondente à Eq. (2.7) com as condições de contorno expressas nas Eqs. (2.9) e (2.10).

Efetuamos cálculos de CC utilizando parâmetros cujos valores simulam, de uma forma restrita, visto que lidamos somente com um problema bidimensional (coordenadas $r$ e $s$ ), a fusão do sistema ${ }^{64} \mathrm{Ni}+{ }^{64} \mathrm{Ni}$. Desta forma, foi fixado $\mu=32.0$ u.m.a., $V_{B}=96.5 \mathrm{MeV}, \hbar \omega=3.7 \mathrm{MeV}, \sigma=0.3$ fm, e $F=5 \mathrm{MeV} / \mathrm{fm}$. A Figura 2.1 mostra o coeficiente de transmissão total para várias energias de excitação. A linha cheia nesta figura representa o resultado para o potencial $b_{a r e}{ }^{1}$, i.e. que foi obtido sem nenhum acoplamento $(F=0)$. Se $V_{B} \gg \hbar \omega$ e $E \gg \hbar \omega$, o coeficiente de transmissão do potencial bare através de uma barreira parabólica de potencial pode ser expresso através da fórmula de Hill-Wheeler [43]:

$$
T=\frac{1}{1+\exp \left[2 \pi\left(V_{B}-E\right) / \hbar \omega\right]}
$$

Como esperado, os acoplamentos provocam um aumento da transmissão (enhancement) em energias abaixo da barreira $\left(V_{B}\right)$ e o enhancement é maior para valores menores de $E^{*}$. No entanto, em energias acima da barreira, os acoplamentos resultam em uma diminuição da transmissão (hindrance). Este efeito não é freqüentemente discutido na literatura, mas já foi observado na fusão de íons pesados (ver e.g. [20, 21]).

\footnotetext{
${ }^{1} \mathrm{O}$ termo bare (do inglês descoberto, desencapado, despido) é usualmente empregado em Física Nuclear para descre-
} 


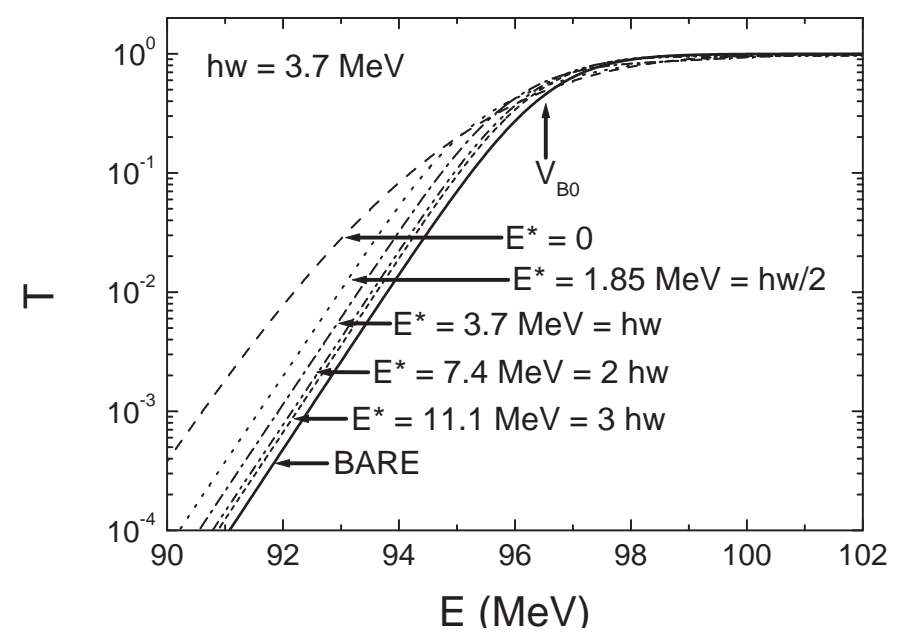

Figura 2.1: Coeficientes de transmissão total, obtidos através de cálculos de CC, como funções da energia cinética para várias energias de excitação. A linha cheia representa os resultados obtidos sem considerar nenhum acoplamento.

O potencial de polarização para o canal elástico é definido como:

$$
V_{\mathrm{pol}}(r)=\frac{1}{\psi_{0}(r)} \sum_{n=1}^{\infty} V_{0 n} \psi_{n}(r)=-\sigma F \frac{\psi_{1}(r)}{\psi_{0}(r)}
$$

de forma que a Eq. (2.7) pode ser reescrita para o canal elástico como:

$$
-\frac{\hbar^{2}}{2 \mu} \frac{d^{2} \psi_{0}}{d r^{2}}+\left[V_{B}-\frac{1}{2} \mu \omega^{2} r^{2}+V_{\mathrm{pol}}(r)\right] \psi_{0}(r)=E \psi_{0}(r)
$$

Comparando as Eqs. (2.7) e (2.15) vemos que, no primeiro caso, para obter a função de onda do canal elástico, devemos resolver um sistema de equações diferenciais acopladas para encontrar as funções de onda de todos os canais, enquanto no segundo basta, conhecendo o potencial de polarização, integrar uma única equação diferencial desacoplada. Obviamente, o potencial de polarização depende, por definição, das próprias soluções para as funções de onda de todos os canais, advindo daí o interesse de propormos modelos que descrevam o comportamento do potencial de polarização.

Na Figura 2.2 são mostradas as partes real e imaginária do potencial de polarização do canal elástico para diversos casos, assumindo valores para os parâmetros de forma a simular o sistema ${ }^{58} \mathrm{Ni}$ $+{ }^{64} \mathrm{Ni}$. Desta forma, foi fixado $\mu=30.4 \mathrm{amu}, V_{B}=98 \mathrm{MeV}, \hbar \omega=3.8 \mathrm{MeV}, \sigma=0.3 \mathrm{fm}$, e $F=5$ 
$\mathrm{MeV} / \mathrm{fm}$. O potencial de polarização apresenta uma complicada estrutura que pode ser reproduzida com uma boa aproximação sem resolver as equações de CC através do método descrito adiante na Seção 2.4 (dentro do contexto da barreira de potencial parabólica).

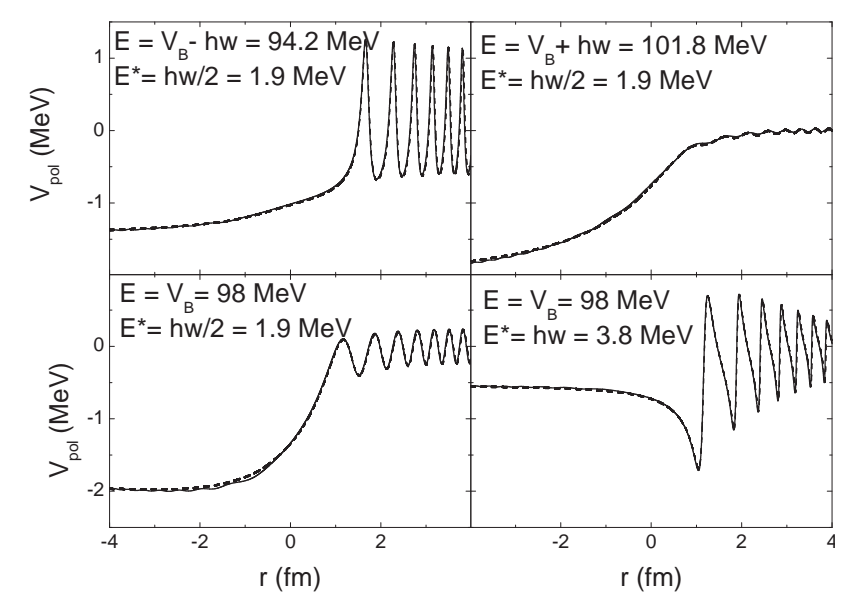

(a) Parte real do potencial de polarização.

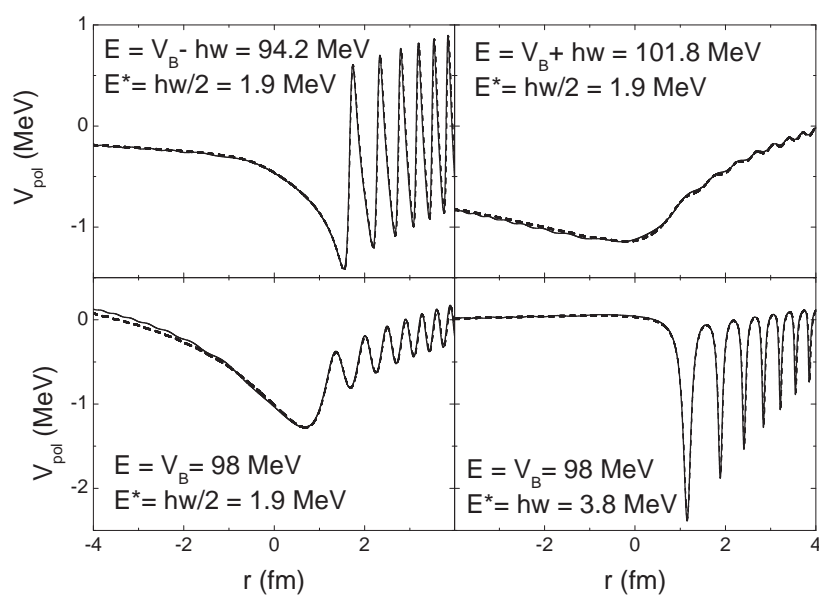

(b) Parte imaginária do potencial de polarização.

Figura 2.2: Partes reais e imaginárias do potencial de polarização obtidas para diferentes valores de energia cinética e de excitação. As linhas cheias representam os resultados exatos de cálculos de CC enquanto as linhas tracejadas correspondem aos resultados do método descrito na Seção 2.4, o modelo proposto no presente trabalho e referido como Movimento de Ponto Zero. As linhas cheias e tracejadas se mostram praticamente indistinguíveis na figura.

\subsection{Aproximação Congelada}

No caso de reações envolvendo núcleos com energia de excitação muito baixa, a chamada Aproximação Congelada (FA, do inglês Frozen Approximation), também conhecida como Aproximação Súbita (Sudden Approximation), se mostra bastante útil. A idéia central do modelo consiste em considerar que a colisão nuclear pode ser calculada como uma média ponderada sobre as diversas deformações possíveis, sendo estas deformações assumidas como estáticas (ou "congeladas") visto que baixas energias de excitação correspondem a baixas freqüências de vibração indicando que determinada configuração deformada pouco ou nada se altera durante o processo de colisão.

Para ilustrar o modelo de FA, consideremos o mesmo espalhamento descrito na Seção 2.1, ou 
seja, a função de onda total do sistema, $\Psi(r, s)$, obedece à equação de Schrödinger expressa na Eq. (2.1) e está sujeita às Hamiltonianas e interações descritas nas Eqs. (2.2) a (2.5).

Definimos, então, a função de onda de FA, $\psi_{F A}(r, s)$, em termos da função de onda de CC, $\Psi(r, s)$, através da expressão:

$$
\Psi(r, s)=\psi_{F A}(r, s)|0\rangle
$$

Inserindo esta expressão na Eq. (2.1) obtemos:

$$
-\frac{\hbar^{2}}{2 \mu} \frac{\partial^{2} \psi_{F A}}{\partial r^{2}}+\left[V(r, s)+V_{\mathrm{PPDS}}(r, s)\right] \psi_{F A}=E \psi_{F A}
$$

onde o potencial de polarização dependente de $s\left(V_{\mathrm{PPDS}}\right)$ é dado por:

$$
V_{\mathrm{PPDS}}(r, s)=-\frac{E^{*} \sigma^{2}}{\psi_{F A}}\left(\frac{\partial^{2} \psi_{F A}}{\partial s^{2}}-\frac{s}{\sigma^{2}} \frac{\partial \psi_{F A}}{\partial s}\right)
$$

O comportamento assintótico de $\psi_{F A}$ é, pelas Eqs. (2.9), (2.10), (2.16) e (2.6):

$$
\begin{gathered}
\psi_{F A}\left(r \geq r_{0}, s\right)=\sum_{n}\left(\delta_{n, 0} e^{-i k_{0} r}+b_{n} e^{i k_{n} r}\right) \frac{H_{n}(s / \sigma \sqrt{2})}{\sqrt{n ! 2^{n}}} \\
\psi_{F A}\left(r \leq-r_{0}, s\right)=\sum_{n} a_{n} e^{-i k_{n} r} \frac{H_{n}(s / \sigma \sqrt{2})}{\sqrt{n ! 2^{n}}}
\end{gathered}
$$

No caso especial de $E^{*}=0$, que é justamente a condição de validade da FA, as Eqs. (2.17), (2.19) e (2.20) se tornam:

$$
\begin{gathered}
-\frac{\hbar^{2}}{2 \mu} \frac{\partial^{2} \psi_{F A}}{\partial r^{2}}+V(r, s) \psi_{F A}=E \psi_{F A}, \\
\psi_{F A}\left(r \geq r_{0}, s\right)=e^{-i k_{0} r}+B_{S} e^{i k_{0} r} \\
\psi_{F A}\left(r \leq-r_{0}, s\right)=A_{S} e^{-i k_{0} r} .
\end{gathered}
$$


com

$$
\begin{aligned}
& A_{S}=\sum_{n} a_{n} \frac{H_{n}(s / \sigma \sqrt{2})}{\sqrt{n ! 2^{n}}} \\
& B_{S}=\sum_{n} b_{n} \frac{H_{n}(s / \sigma \sqrt{2})}{\sqrt{n ! 2^{n}}} .
\end{aligned}
$$

Usando o fato de que o coeficiente de transmissão pode ser escrito como:

$$
T=\int_{-\infty}^{+\infty}-i \frac{\Psi\left(r \leq r_{0}, s\right)}{k_{0}} \frac{\partial \Psi^{*}\left(r \leq r_{0}, s\right)}{\partial r} d s
$$

obtemos, substituindo a Eq. (2.23) na Eq. (2.26):

$$
\left.T=\left\langle 0\left|T_{S}\right| 0\right\rangle=\int_{-\infty}^{+\infty} T_{S}|| 0\right\rangle\left.\right|^{2} d s
$$

onde $T_{S}=\left|A_{S}\right|^{2}$. Os coeficientes de transmissão dependentes de $s\left(T_{S}\right)$ podem ser obtidos através da fórmula de Hill-Wheeler (Eq. (2.13)) com a altura da barreira parabólica deslocada: $V_{B}-F s$. Para cada valor de $s$, as Eqs. (2.21) a (2.23) podem representar o espalhamento de uma partícula por uma barreira desacoplada, enquanto a Eq. (2.27) mostra que o coeficiente de transmissão total pode ser obtido simplesmente por uma média das transmissões parciais dependentes de $s\left(T_{S}\right)$ sobre as configurações congeladas na coordenada $s$. Obviamente, este resultado é esperado visto que, para $E^{*}=0$, a frequiência de vibração se anula. Deve-se notar que, neste limite, a FA é totalmente equivalente ao formalismo de CC, levando em conta o efeito do conjunto completo e infinito de estados acoplados. Para $E^{*} \neq 0$, a Eq. (2.27) ainda é valida desde que a Eq. (2.17) seja resolvida considerando-se o comportamento assintótico exato dado pelas Eqs. (2.19) e (2.20). Neste caso, porém, os coeficientes $a_{n} \mathrm{e}$ $b_{n}$ e $V_{\text {PPDS }}$ contidos nestas equações podem somente ser obtidos resolvendo as equações acopladas, de forma que a FA não é mais útil. Apesar disso, na Seção 2.3 são propostas aproximações que fornecem, dentro do contexto da FA, bons resultados para o coeficiente de transmissão para $E^{*} \neq 0$, especialmente para energias bem abaixo da altura da barreira. 


\subsection{FA Generalizada para Energia de Excitação Finita}

Dentro de um contexto similar ao da FA, descrita na Seção 2.2, nós desenvolvemos uma aproximação que leva em conta os efeitos de energia de excitação finita $\left(E^{*} \neq 0\right)$, particularmente útil para o cálculo da probabilidade de transmissão por uma barreira, associada à seção de choque de fusão. Assim como na FA, esta Aproximação Congelada Generalizada (GFA, do inglês Generalized Frozen Approximation) considera [37] os acoplamentos à toda banda vibracional sem resolver as equações de CC. Nesta seção descreveremos o modelo de GFA aplicado à mesma barreira parabólica e acoplamento linear já utilizados nas seções anteriores, ou seja, Eqs. (2.1) a (2.5).

Consideramos inicialmente que a parte real do potencial de polarização dependente de $s$, definido na Eq. (2.18), deve desempenhar um papel mais importante que a parte imaginária ao se calcular coeficientes de transmissão, visto que a parte real afeta diretamente a barreira parabólica de potencial. Baseado neste argumento, a parte imaginária de $V_{\mathrm{PPDS}}$ é desprezada no desenvolvimento do modelo da GFA.

A Figura 2.3(a) apresenta a parte real do potencial de polarização dependente de $s$ em diversos casos. Na Figura 2.3(b) é mostrada (linha cheia) a soma do $V_{\text {PPDS }}$ e o correspondente $V(r, s)$. As linhas tracejadas na Figura 2.3(b) correspondem simplesmente a $V(r, s)$. Como pode ser observado, o efeito principal de $V_{\mathrm{PPDS}}$ consiste apenas em uma mudança na altura da barreira, $\Delta V_{B}(s)$. Esta mudança pode ser estimada no topo da barreira, i.e.: $\Delta V_{B}(s) \approx \operatorname{Real}\left[V_{\mathrm{PPDS}}(r=0, s)\right]$. Logo, os valores de $T_{S}$ e, conseqüentemente, a transmissão total da Eq. (2.27), podem ser obtidos considerando o tunelamento através de uma barreira parabólica efetiva, dada por:

$$
V_{\mathrm{ef}}(r, s)=V(r, s)+\Delta V_{B}(s)=V_{B_{\mathrm{ef}}}(s)-\frac{1}{2} \mu \omega^{2} r^{2}
$$

onde $V_{B_{\text {ef }}}(s)=V_{B}-F s+\Delta V_{B}(s)$ é a altura efetiva da barreira.

Para obter boas estimativas para o coeficiente de transmissão dentro do contexto da GFA, sem resolver as equações acopladas, é necessário uma estimativa para $\Delta V_{B}(s)$. O efeito mais importante dos acoplamentos na probabilidade de transmissão ocorre na região de energia abaixo da barreira, 


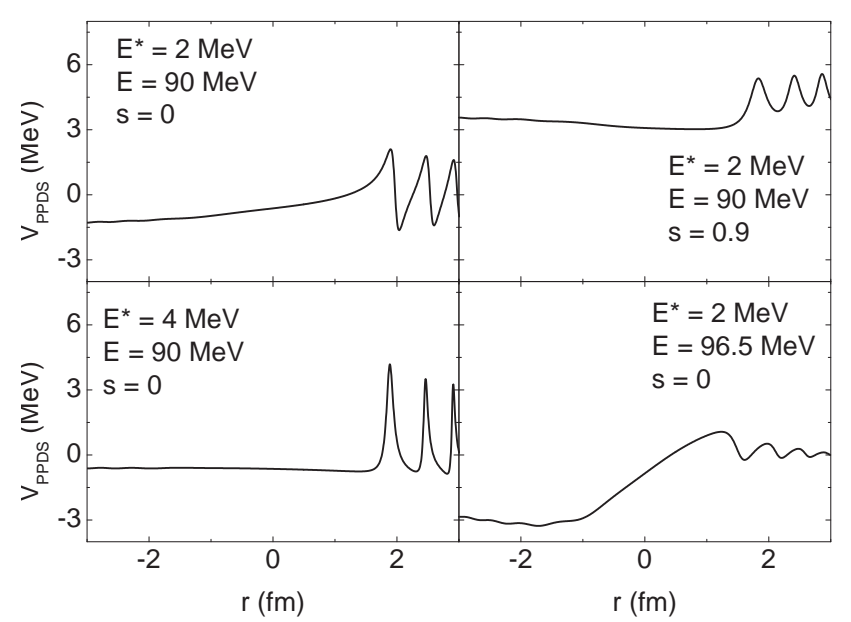

(a) Parte real de $V_{\text {PPDS }}$.

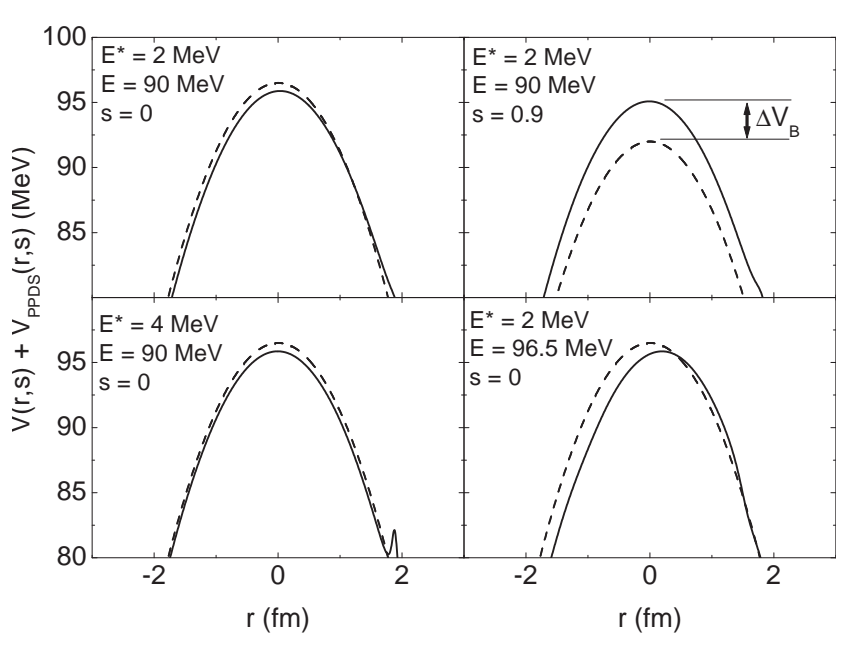

(b) Soma da parte real de $V_{\mathrm{PPDS}} \operatorname{com} V(r, s)$.

Figura 2.3: Parte real de $V_{\mathrm{PPDS}}($ a) e a soma deste com $V(r, s)$ (linhas cheias) (b), obtido em diferentes condições. As linhas tracejadas em (b) correspondem somente à $V(r, s)$. Em um dos casos, a mudança na altura da barreira $\left(\Delta V_{B}\right)$ é indicada na figura.

onde a aproximação WKB pode ser assumida para a função de onda:

$$
\psi_{F A}(r=0, s) \approx e^{-\pi\left[V_{B}(s)-E\right] / 2 \hbar \omega} .
$$

Inserindo a Eq. (2.29) na Eq. (2.18) e extraindo a parte real de $V_{P P D S}$, obtém-se a equação:

$$
\Delta V_{B}(s)=-E^{*} \sigma^{2}\left[\left(\frac{\pi}{2 \hbar \omega}\right)^{2}\left(\frac{\partial \Delta V_{B}}{\partial s}-F\right)^{2}-\frac{\pi}{2 \hbar \omega} \frac{\partial^{2} \Delta V_{B}}{\partial s^{2}}+\frac{\pi s}{2 \hbar \omega \sigma^{2}}\left(\frac{\partial \Delta V_{B}}{\partial s}-F\right)\right]
$$

Uma solução em primeira ordem em $s$ é:

$$
\Delta V_{B}(s)=-E^{*}\left[\frac{\pi \sigma F}{2 \hbar \omega+\pi E^{*}}\right]^{2}+s F \frac{\pi E^{*}}{2 \hbar \omega+\pi E^{*}}
$$

Assumindo a Eq. (2.31) e substituindo $V_{B}$ por $V_{B_{\mathrm{ef}}}(s)$ pode-se imediatamente calcular os valores de $T_{S}$ através da fórmula de Hill-Wheeler (Eq. (2.13)). O coeficiente de transmissão total é então obtido a partir da Eq. (2.27).

De forma a testar o modelo da FA generalizada (GFA), os resultados correspondentes (linhas 
cheias) foram comparados com os obtidos de cálculos de CC (linhas tracejadas) para diversas energias de excitação nas Figuras 2.4(a) e 2.4(b). A título de comparação, as linhas pontilhadas nas figuras representam os resultados do potencial bare. Como pode ser visto na Figura 2.4(a), o modelo de FA generalizada produz resultados bastante similares em comparação com os cálculos de CC em energias abaixo da barreira. Por outro lado, em energias em torno de $V_{B}$ é observada uma pequena discordância (ver Figura 2.4(b)). Obviamente este comportamento deveria ser esperado, visto que na dedução de $\Delta V_{B}$ (Eq. (2.31)) a função de onda em baixas energias foi assumida como válida. No entanto, em energias muito altas, deve-se obter $\Delta V_{B}=0$. De fato, se a energia cinética no topo da barreira é muito maior do que a energia de excitação, a FA original deve ser apropriada pois o "tempo de tunelamento" é muito menor que o período de vibração. Logo, para $E-V_{B} \gg E^{*}$ deve-se assumir que $\Delta V_{B}=0$. Portanto, de forma a melhorar o modelo, o seguinte ansatz é proposto:

$$
\begin{gathered}
\Delta V_{B}(s)=(C-1) E^{*}\left[\left(\frac{\pi \sigma F}{2 \hbar \omega+\pi E^{*}}\right)^{2}-\frac{\pi s F}{2 \hbar \omega+\pi E^{*}}\right] \\
C=\frac{1}{1+e^{2 \pi\left(V_{B}-E+E^{*} / 4\right) / \hbar \omega}}
\end{gathered}
$$

A Figura 2.4(c) mostra os resultados obtidos para os coeficientes de transmissão a partir da Eq. (2.32). $\mathrm{Na}$ região de energias abaixo da barreira, a Eq. (2.32) fornece resultados bastante similares aos obtidos a partir da Eq. (2.31). Em energias próximas à barreira, a Eq. (2.32) claramente produz resultados melhores (comparando com cálculos de CC) do que a Eq. (2.31). Deve ser ressaltado que o modelo também foi testado com sucesso para diferentes combinações de valores para $\mu, \sigma, F, V_{B} \mathrm{e}$ $\hbar \omega$, correspondendo à sistemas bem diferentes de íons pesados.

Apesar de o modelo ter sido baseado principalmente na função de onda na região de energia cinética baixa, é interessante observar que a Eq. (2.32) reproduz o comportamento esperado dos coeficientes de transmissão em diversos outros casos assintóticos. Por exemplo, para energias de excitação desprezíveis, obtemos $\Delta V_{B} \approx 0$, e o modelo de FA generalizada reduz-se à FA original, como deveria. Por outro lado, para $E^{*} \rightarrow \infty$, obtém-se $V_{B_{e f}}(s)=V_{B}$ e nenhum efeito dos acoplamentos é observado. Este é o comportamento esperado pois, para valores muito altos de $E^{*}$, a frequiência de vibração é também muito alta. O sistema deveria então "sentir" um potencial médio, que é simplesmente o potencial bare visto que o termo de acoplamento é linear $(F s)$. Finalmente, 
para $E-V_{B} \gg E^{*}$ a correção $(C-1)$ introduzida na Eq. (2.32) garante que o modelo forneça resultados similares aos da FA, como deveria ser. Portanto, o modelo de FA generalizada é totalmente consistente em todos os limites assintóticos: $E \ll V_{B}, E \gg V_{B}+E^{*}, E^{*} \rightarrow 0$, e $E^{*} \rightarrow \infty$. A principal limitação do modelo ocorre para $E^{*}$ moderado, na região de energia $E \approx V_{B}$. Mesmo assim, resultados razoáveis também são obtidos neste caso (ver Figura 2.4(c)).

Com o objetivo de generalizar o modelo, consideremos um conjunto de $N$ diferentes graus de liberdade vibracionais acoplados à barreira parabólica de uma forma completamente independente. A Hamiltoniana pode, então, ser representada como:

$$
\begin{gathered}
{\left[H_{0}+\sum_{i=1}^{N} H_{i}\right] \Psi(r, s)=\left(E+\sum_{i} E_{i}^{*} / 2\right) \Psi(r, s)} \\
H_{i}=-\frac{\hbar^{2}}{2 D_{i}} \frac{\partial^{2}}{\partial s_{i}^{2}}+\frac{1}{2} D_{i}\left(E_{i}^{*} / \hbar\right)^{2} s_{i}^{2} \\
V\left(r, s_{i}\right)=V_{B}-\sum_{i=0}^{N} F_{i} s_{i}-\frac{1}{2} \mu \omega^{2} r^{2} ;-r_{0} \leq r \leq r_{0}
\end{gathered}
$$

onde $H_{i}$ é a Hamiltoniana que representa, independentemente, cada um dos graus de liberdade vibracionais, assumidos como osciladores harmônicos. O coeficiente total de transmissão pode agora ser obtido por:

$$
T=\left\langle 0_{1}, 0_{2}, \ldots, 0_{N}\left|T_{S}\right| 0_{1}, 0_{2}, \ldots, 0_{N}\right\rangle
$$

onde cada $T_{S}$ deve ser calculado através da expressão de Hill-Wheeler (Eq. (2.13)) considerando a correspondente altura efetiva da barreira:

$$
V_{B_{e f}}=V_{B}-\sum_{i=0}^{N}\left[F_{i} s_{i}-\Delta V_{B}\left(s_{i}\right)\right]
$$

com cada $\Delta V_{B}\left(s_{i}\right)$ obtido a partir da Eq. (2.32) através dos correspondentes $\sigma_{i}, F_{i}$ e $E_{i}^{*}$. 


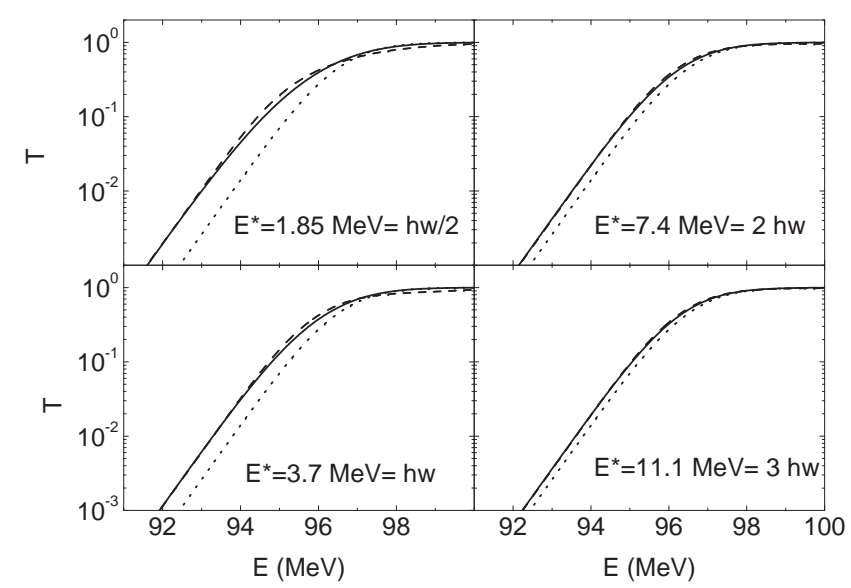

(a) Assumindo $\Delta V_{B}$ dado pela Eq. (2.31).

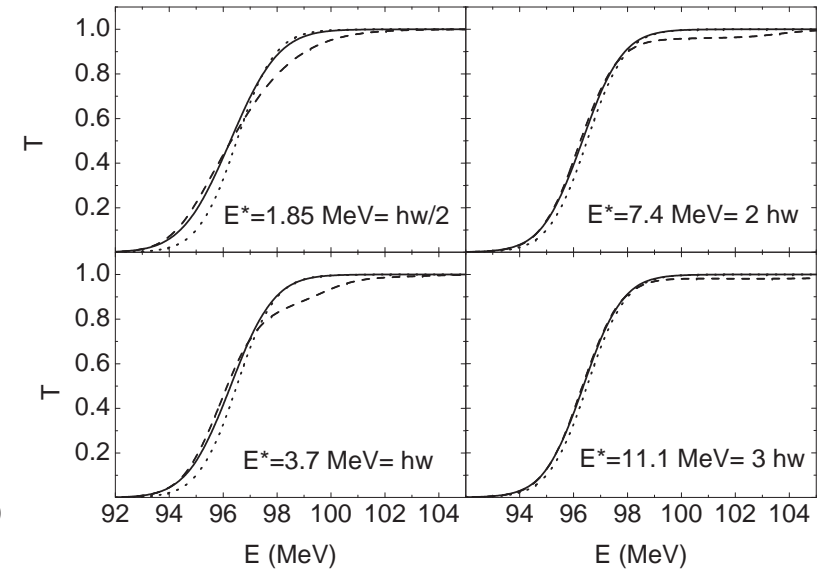

(b) Assumindo também $\Delta V_{B}$ dado pela Eq. (2.31), porém em escala linear para ilustrar a região da barreira, $E \approx V_{B}=96.5 \mathrm{MeV}$.

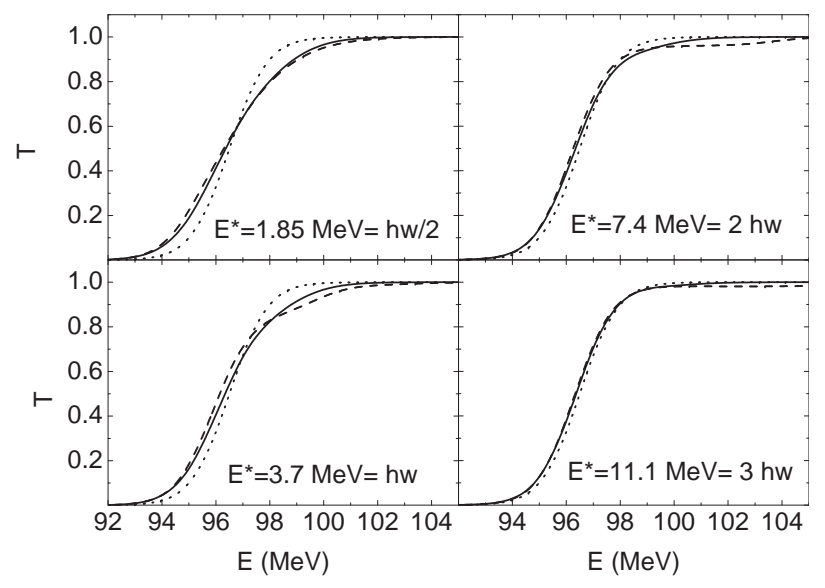

(c) Assumindo $\Delta V_{B}$ dado pela Eq. (2.32), também em escala linear para ilustrar a região da barreira, $E \approx V_{B}=96.5 \mathrm{MeV}$.

Figura 2.4: Coeficientes de transmissão obtidos de cálculos de CC (linhas tracejadas) e modelo de $F A$ generalizada (linhas cheias), assumindo que $\Delta V_{B}$ é representado pela Eq. (2.31) (a,b) ou pela Eq. (2.32) (c). Os cálculos foram feitos para diversos valores de energia de excitação. Na figura, as linhas pontilhadas representam os resultados obtidos sem considerar qualquer acoplamento. 


\subsection{Modelo do Movimento de Ponto Zero}

Em um trabalho de Dasso, Landowne e Winter [14], foi desenvolvido um modelo para a obtenção do coeficiente de transmissão através de uma barreira parabólica de potencial de altura $V_{B}$ e curvatura $\hbar \omega$, incluindo os efeitos da energia de excitação, considerando uma aproximação para a função de onda assintótica. Foi encontrado que:

$$
T=\sum_{n} W_{n} T_{n}
$$

onde os fatores de peso são

$$
W_{n}=\frac{1}{n !}\left(\frac{F \sigma}{E^{*}}\right)^{2 n} e^{-\left(F \sigma / E^{*}\right)^{2}},
$$

e os coeficientes de transmissão parcial podem ser obtidos através da expressão de Hill-Wheeler (Eq. (2.13)) com a altura da barreira modificada para cada $n$ :

$$
T_{n}=\frac{1}{1+\exp \left[2 \pi\left(V_{B}-E+\lambda_{n}\right) / \hbar \omega\right]},
$$

considerando os correspondentes autovalores

$$
\lambda_{n}=n E^{*}-(F \sigma)^{2} / E^{*}
$$

O efeito dos acoplamentos é substituir a altura da barreira $V_{B}$ por um conjunto de barreiras $V_{B}+\lambda_{n}$, e o coeficiente de transmissão total é dado por uma média ponderada das transmissões para cada barreira efetiva. Na Ref. [14], o caso especial do problema de apenas dois canais também foi estudado. Neste caso, os correspondentes autovalores e fatores de peso são obtidos:

$$
\begin{gathered}
\lambda_{ \pm}=\frac{1}{2}\left[E^{*} \pm \sqrt{\left(E^{*}\right)^{2}+4(F \sigma)^{2}}\right] \\
W_{ \pm}=\frac{2(F \sigma)^{2}}{4(F \sigma)^{2}+\left(E^{*}\right)^{2} \pm E^{*} \sqrt{\left(E^{*}\right)^{2}+4(F \sigma)^{2}}} .
\end{gathered}
$$

Com o intuito de avaliar os modelos até aqui apresentados, na Figura 2.5 são mostrados resultados de cálculos de CC, FA generalizada (descrita na Seção 2.3) e também aqueles obtidos através da Eq. 
(2.39). As linhas pontilhadas na figura representam o resultado do potencial bare. Tanto o modelo GFA como a Eq. (2.39) fornecem resultados muito bons em energias abaixo da barreira (Figura 2.5 - embaixo). Resultados bastante razoáveis, porém ligeiramente diferentes, são obtidos por ambos os modelos em energias em torno da barreira (Figura 2.5 - em cima).

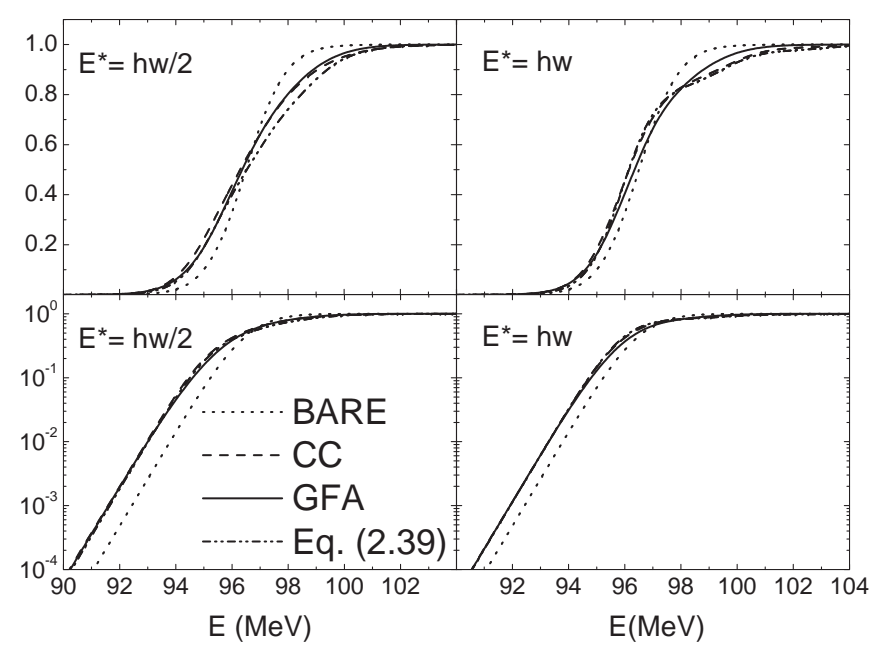

Figura 2.5: A figura apresenta uma comparação entre os resultados para o coeficiente de transmissão total obtidos dos modelos de CC (Seção 2.1), GFA (Seção 2.3), Eq. (2.39) e somente potencial bare, para duas energias de excitação diferentes. Os resultados são apresentados escala linear (em cima) $e$ logarítmica (embaixo).

Com base no modelo descrito na Ref. [14], propomos uma generalização que possibilita o cálculo da probabilidade transmissão e que permite obter, também, o potencial de polarização e, conseqüentemente, o coeficiente de reflexão. Denominamos este modelo Movimento de Ponto Zero (ZPM, do inglês Zero Point Motion).

É possível desacoplar as Eqs. (2.1) a (2.5) se for considerada a seguinte mudança de variáveis:

$$
u=s-\frac{2 F \sigma^{2}}{E^{*}}
$$

o que resulta:

$$
H_{o s c}(s)-s F=H_{o s c}(u)-\frac{(F \sigma)^{2}}{E^{*}}
$$

Expandindo a função de onda total de canais acoplados na base de auto-vetores de $H_{o s c}(u), \Psi(r, s)=$ 
$\sum_{m} Y_{m}(r)|m(u)\rangle$, a Eq. (2.1) se torna:

$$
\left[-\frac{\hbar^{2}}{2 \mu} \frac{\partial^{2}}{\partial r^{2}}+V_{B}-\frac{1}{2} \mu \omega^{2} r^{2}+m E^{*}-\frac{(F \sigma)^{2}}{E^{*}}\right] Y_{m}(r)=E Y_{m}(r)
$$

As funções de onda dependentes de $r$ das duas bases, $s$ e $u$, relacionam-se entre si através das expressões:

$$
\begin{aligned}
& \psi_{n}(r)=\sum_{m}\langle n(s) \mid m(u)\rangle Y_{m}(r)=\sum_{m} U_{m n} Y_{m}(r) \\
& Y_{m}(r)=\sum_{n}\langle m(u) \mid n(s)\rangle \psi_{n}(r)=\sum_{n} U_{m n} \psi_{n}(r),
\end{aligned}
$$

onde os elementos de matriz de projeção entre as bases são dados por:

$$
U_{m n}=e^{-\frac{1}{2}\left(\frac{F \sigma}{E^{*}}\right)^{2}} \sum_{j=0}^{m i n(n, m)}(-1)^{(m-j)} \frac{\sqrt{m ! n !}\left(\frac{F \sigma}{E^{*}}\right)^{m+n-2 j}}{j !(m-j) !(n-j) !}
$$

A dedução analítica detalhada da Eq. (2.50) encontra-se no Apêndice B.

Aplicando as Eqs. (2.9) e (2.10) na Eq. (2.49), e assumindo a aproximação $k_{n} \approx k_{0}$, podemos obter o comportamento assintótico de $Y_{m}(r)$ :

$$
\begin{gathered}
Y_{m}\left(r \geq r_{0}\right)=U_{m 0} e^{-i k_{0} r}+\sum_{n} U_{m n} b_{n} e^{i k_{0} r}, \\
Y_{m}\left(r \leq-r_{0}\right)=\sum_{n} U_{m n} a_{n} e^{-i k_{0} r} .
\end{gathered}
$$

Definimos agora a função de onda $\bar{Y}_{m}(r) \equiv U_{m 0}^{-1} Y_{m}(r)$, que também obedece à Eq. (2.47), mas tem o seguinte comportamento assintótico:

$$
\begin{gathered}
\bar{Y}_{m}\left(r \geq r_{0}\right)=e^{-i k_{0} r}+\bar{b}_{m} e^{i k_{0} r} \\
\bar{Y}_{m}\left(r \leq-r_{0}\right)=\bar{a}_{m} e^{-i k_{0} r}
\end{gathered}
$$

onde

$$
\bar{a}_{m}=U_{m 0}^{-1} \sum_{n} U_{m n} a_{n}
$$




$$
\bar{b}_{m}=U_{m 0}^{-1} \sum_{n} U_{m n} b_{n}
$$

As Eqs. (2.53) e (2.54), ligadas à Eq. (2.47) para $\bar{Y}$, correspondem a equações simples e desacopladas de barreiras parabólicas cuja altura é modificada, sendo agora dada por $V_{B}+m E^{*}-$ $(F \sigma)^{2} / E^{*}$. A função de onda correspondente, $\bar{Y}_{m}(r)$, pode ser obtida através das séries analíticas descritas no Apêndice C, pelas expressões obtidas utilizando-se a aproximação WKB (Apêndice D) ou simplesmente por integração numérica. Invertendo a Eq. (2.55) e considerando a expressão de Hill-Wheeler (Eq. (2.13)) como uma boa aproximação para $\left|\bar{a}_{m}\right|^{2}$, obtém-se o coeficiente de transmissão total dado pelas Eqs. (2.39) a (2.42).

Finalmente, combinando as Eqs. (2.14), (2.48) e $\bar{Y}_{m}(r)=U_{m 0}^{-1} Y_{m}(r)$, é possível obter o potencial de polarização:

$$
V_{p o l}(r)=-F \sigma \frac{\sum_{m} U_{m 1} U_{m 0} \bar{Y}_{m}(r)}{\sum_{m} U_{m 0}^{2} \bar{Y}_{m}(r)}
$$

Da Eq. (2.50), pode-se obter que $U_{m 0}^{2}=W_{m}$ da Eq. (2.40), que corresponde a uma distribuição de Poisson com valor médio e desvio padrão dados por: $\langle m\rangle=\left(F \sigma / E^{*}\right)^{2}, \sigma_{m}=\sqrt{\langle m\rangle}$, respectivamente. Por outro lado: $U_{m 1} U_{m 0}=W_{m}(\langle m\rangle-m) / \sigma_{m}$. Logo, a Eq. (2.47) para $\bar{Y}$ pode ser reescrita de outra forma:

$$
\left[-\frac{\hbar^{2}}{2 \mu} \frac{\partial^{2}}{\partial r^{2}}+V_{B}-\frac{1}{2} \mu \omega^{2} r^{2}+F \sigma\left(\frac{m-\langle m\rangle}{\sigma_{m}}\right)\right] \bar{Y}_{m}(r)=E \bar{Y}_{m}(r)
$$

De forma a testar o modelo para o potencial de polarização, comparamos os resultados correspondentes (linhas tracejadas) com cálculos de CC (linhas cheias) para vários valores de energia de excitação na Figura 2.2 (página 10). Como pode ser visto na Figura 2.2, o modelo produz resultados bastante similares em comparação com cálculos de CC.

Neste ponto temos um método para a obtenção do potencial de polarização sem resolver as equações de CC. Logo, a Eq. (2.15) pode ser resolvida como uma equação desacoplada, visto que a polarização já contém os efeitos dos acoplamentos. Obviamente, as funções de onda assintóticas correspondentes são representadas pelas Eqs. (2.9) e (2.10), com $n=0$. Logo, de maneira a testar a precisão do modelo de ZPM, resolvemos a Eq. (2.15) com a estimativa aproximada para a polarização, Eq. (2.57), e calculamos os coeficientes de reflexão para o canal elástico. A Figura 2.6 compara 
os resultados obtidos por este método com os cálculos exatos de CC completo para duas energias de excitação. Estes resultados são muito similares, com pequenas discordâncias para energias acima da barreira $\left(V_{B}=98 \mathrm{MeV}\right)$ onde o coeficiente de reflexão é muito pequeno.

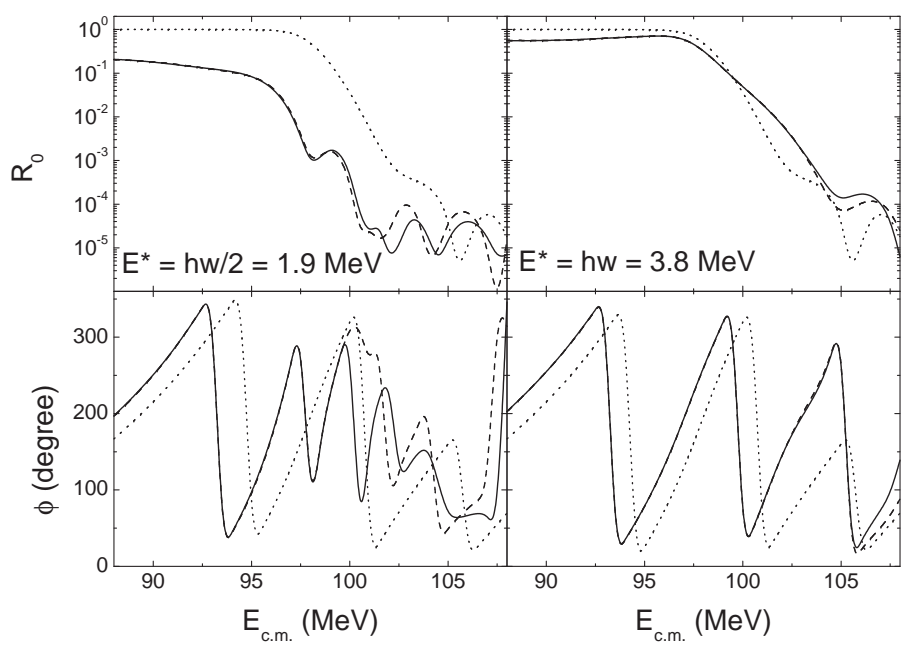

Figura 2.6: Coeficientes de reflexão do canal elástico $\left(R_{0}=\left|b_{0}\right|^{2}\right)$, em cima, e fase de $b_{0}$, embaixo, para duas energias de excitação. As linhas cheias representam os resultados exatos de CC. As linhas tracejadas correspondem aos resultados obtidos resolvendo a Eq. (2.15) desacoplada, com o potencial de polarização obtido de acordo com o método de ZPM. Como comparação, as linhas pontilhadas na figura representam os resultados sem considerar nenhum acoplamento.

Estes resultados podem ser facilmente generalizados para um conjunto de diferentes graus de liberdade vibracionais acoplados à barreira parabólica de uma forma independente. Neste trabalho, por exemplo, será considerado o caso de quatro graus de liberdade e, neste contexto, obtemos:

$$
T=\sum_{m_{1}} \sum_{m_{2}} \sum_{m_{3}} \sum_{m_{4}} W_{m_{1}}^{(1)} W_{m_{2}}^{(2)} W_{m_{3}}^{(3)} W_{m_{4}}^{(4)} T_{m}
$$

onde os fatores de peso são:

$$
W_{m}^{(j)}=\frac{1}{m !}\left(\frac{F_{j} \sigma_{j}}{E_{j}^{*}}\right)^{2 m} e^{-\left(F_{j} \sigma_{j} / E_{j}^{*}\right)^{2}}
$$


e os coeficientes parciais de transmissão podem ser obtidos através da expressão de Hill-Wheeler:

$$
T_{m}=\frac{1}{1+\exp \left[2 \pi \frac{\left(V_{B}-E+\lambda_{m_{1}}^{(1)}+\lambda_{m_{2}}^{(2)}+\lambda_{m_{3}}^{(3)}+\lambda_{m_{4}}^{(4)}\right)}{\hbar \omega}\right]}
$$

considerando os correspondentes autovalores:

$$
\lambda_{m}^{(j)}=m E_{j}^{*}-\left(F_{j} \sigma_{j}\right)^{2} / E_{j}^{*}
$$

O potencial de polarização pode ser obtido através de:

$$
\begin{gathered}
V_{\mathrm{pol}}(r)=-\frac{F_{1} \sigma_{1} \psi_{1}+F_{2} \sigma_{2} \psi_{2}+F_{3} \sigma_{3} \psi_{3}+F_{4} \sigma_{4} \psi_{4}}{\psi_{0}} \\
\psi_{0}=\sum_{m_{1}} \sum_{m_{2}} \sum_{m_{3}} \sum_{m_{4}}\left(U_{m_{1} 0}^{(1)}\right)^{2}\left(U_{m_{2} 0}^{(2)}\right)^{2}\left(U_{m_{3} 0}^{(3)}\right)^{2}\left(U_{m_{4} 0}^{(4)}\right)^{2} \bar{Y}_{m_{1} m_{2} m_{3} m_{4}},
\end{gathered}
$$

e os outros termos $\psi_{i}$ são similares a:

$$
\psi_{2}=\sum_{m_{1}} \sum_{m_{2}} \sum_{m_{3}} \sum_{m_{4}}\left(U_{m_{1} 0}^{(1)}\right)^{2} U_{m_{2} 1}^{(2)} U_{m_{2} 0}^{(2)}\left(U_{m_{3} 0}^{(3)}\right)^{2}\left(U_{m_{4} 0}^{(4)}\right)^{2} \bar{Y}_{m_{1} m_{2} m_{3} m_{4}}
$$

As funções $\bar{Y}(r)$ têm comportamento assintótico de canal único e obedecem a seguinte equação:

$$
\left[-\frac{\hbar^{2}}{2 \mu} \frac{\partial^{2}}{\partial r^{2}}+V_{B}-\frac{1}{2} \mu \omega^{2} r^{2}+\sum_{i=1}^{4} F_{i} \sigma_{i}\left(\frac{m_{i}-\left\langle m_{i}\right\rangle}{\sigma_{m_{i}}}\right)\right] \bar{Y}=E \bar{Y}
$$

onde $\left\langle m_{i}\right\rangle=\left(F_{i} \sigma_{i} / E_{i}^{*}\right)^{2}, \sigma_{m_{i}}=\sqrt{\left\langle m_{i}\right\rangle}$.

Propusemos, neste capítulo, dois diferentes modelos para obter coeficientes de transmissão associados a uma barreira parabólica acoplada a um ou mais graus de liberdade de oscilador harmônico. Um dos métodos também permite calcular de forma bastante acurada o potencial de polarização sem resolver as equações de CC correspondentes. Os modelos são bastante gerais e poderiam ser também aplicados em diversas áreas da Física. Nos próximos capítulos, veremos como adaptá-los ao estudo de colisões entre íons pesados. 


\section{Capítulo 3}

\section{Potenciais e Deformações}

As forças entre objetos extensos é um assunto importante em colisões de íons pesados, onde o potencial de convolução dupla (double-folding) desempenha um papel fundamental na descrição correspondente da interação. A convolução de duas distribuições esfericamente simétricas pode ser facilmente calculada usando-se a representação da transformada de Fourier [44]. Entretanto, ao lidar com distribuições deformadas, a obtenção do potencial se torna uma tarefa árdua devido à resolução numérica da correspondente integral em seis dimensões, que consome muito tempo computacional. Este problema é relevante pois a maioria dos núcleos apresenta deformações permanentes e/ou vibracionais. Diversos artigos apresentaram expressões aproximadas para a interação coulombiana entre núcleos deformados (e.g. Refs. [45, 46]) mas, em geral, tais expressões foram obtidas apenas para grandes distâncias e também assumindo difusividade nula para a distribuição. O potencial nuclear também foi obtido (e.g. Ref. [47]) mas, novamente, de uma forma aproximada.

No presente capítulo é fornecido um modelo que desenvolvemos para obter expressões aproximadas, mas bastante acuradas, para o potencial coulombiano entre dois núcleos deformados com difusividade finita (Seção. 3.3). Os resultados correspondentes são comparados com aqueles obtidos a partir da resolução da integral hexa-dimensional. Também apresentamos neste capítulo, na Seção 3.4, uma maneira de se calcular o potencial nuclear acuradamente, baseada em um modelo de alcance zero $^{1}$ que propusemos recentemente [19]. Inicialmente, porém, são mostrados resumidamente os resultados obtidos para uma sistemática de densidades nucleares, publicados na Ref. [19]. Em

\footnotetext{
${ }^{1}$ Também conhecido pelo temo em inglês Zero-Range Approach
} 
seguida é apresentada uma interessante sistemática que realizamos para os parâmetros de deformação de quadrupolo e octupolo de núcleos pesados.

\subsection{Sistemática de Densidades Nucleares}

Para se calcular a interação efetiva entre dois núcleos é importante o conhecimento das respectivas densidades nucleares de carga e de matéria, visto que os potenciais coulombiano e nuclear podem ser construídos a partir da convolução entre estas. Na Ref. [19], com o objetivo de descrever as densidades de prótons, nêutrons, nucleons (prótons + nêutrons), carga e matéria, foi adotada a distribuição biparamétrica de Fermi ( $2 \mathrm{pF})$, que também já foi amplamente usada para densidades de carga extraídas de experimentos de espalhamento de elétrons [48]. A forma da distribuição $2 \mathrm{pF}$, dada pela equação:

$$
\rho(r)=\frac{\rho_{0}}{1+\exp \left(\frac{r-R_{0}}{a}\right)}
$$

é particularmente apropriada para a descrição de densidades nucleares devido ao achatamento da região interna, que é associado à saturação do meio nuclear, e à queda abrupta (relacionado ao parâmetro de difusividade $a$ ) trazendo a noção do raio $R_{0}$ do núcleo. Os parâmetros $\rho_{0}, a$ e $R_{0}$ são conectados pela condição de normalização:

$$
4 \pi \int \rho(r) r^{2} d r=X
$$

onde $X$ pode ser o número de prótons $Z$, nêutrons $N$ ou nucleons $A=N+Z$. Nos cálculos teóricos, a distribuição de carga do núcleo $\left(\rho_{c}\right)$, a partir da qual obtém-se o potencial coulombiano, pode ser obtida pela convolução da distribuição de prótons do núcleo $\left(\rho_{p}\right)$ com a distribuição de carga intrínseca do próton $\left(\rho_{c p}\right)$ :

$$
\rho_{c}(\vec{r})=\int \rho_{p}\left(\overrightarrow{r^{\prime}}\right) \rho_{c p}\left(\vec{r}-\overrightarrow{r^{\prime}}\right) d r^{\prime}
$$

onde $\rho_{c p}$ é uma exponencial com difusividade $a_{c p}=0.235 \mathrm{fm}$. De maneira análoga, a densidade de matéria do núcleo $\left(\rho_{M}\right)$, a partir da qual obtém-se o potencial nuclear, pode ser definida como a convolução da distribuição de nucleons do núcleo $\left(\rho_{N}\right)$ com a distribuição de matéria intrínseca 
do nucleon $\left(\rho_{m}\right)$, que é assumida como tendo a mesma forma da distribuição de carga intrínseca do próton:

$$
\rho_{M}(\vec{r})=\int \rho_{N}\left(\overrightarrow{r^{\prime}}\right) \rho_{m}\left(\vec{r}-\overrightarrow{r^{\prime}}\right) d \overrightarrow{r^{\prime}}
$$

Por conveniência, as distribuições de carga e matéria são normalizadas ao número de prótons $(Z)$ e de nucleons $(A)$, respectivamente. Usualmente, na literatura, distribuições de matéria e de nucleons têm o mesmo significado. Nós, porém, estabelecemos uma diferença entre essas distribuições, considerando o tamanho finito do nucleon.

Após sistematização das densidades experimentais e teóricas, calculadas para um grande número de núcleos usando o modelo de Dirac-Hartree-Bogoliubov (DHB) [49], foi obtido [19] o comportamento médio, experimental e teórico, das difusividades (ver Figura 3.1) e dos raios (ver Figura 3.2) de carga, de prótons, de nêutrons, de nucleons e de matéria, em função de $Z$ ou $A$, conforme o caso. Após ajuste foi obtido que os valores médios das difusividades das densidades de carga e de matéria correspondem a $a_{c}=0.53 \mathrm{fm}$ e $a=0.56 \mathrm{fm}$, respectivamente, e o valor médio da difusividade da distribuição de nucleons obtido foi de $a_{N}=0.50 \mathrm{fm}$ (para detalhes, ver Ref. [19]). O raio de carga pode, então, ser dado por:

$$
R_{c}=1.76 Z^{1 / 3}-0.96 \mathrm{fm}
$$

onde $Z$ é o número de prótons do núcleo, enquanto o raio de matéria ou o de nucleons corresponde à expressão:

$$
R_{0}=1.31 A^{1 / 3}-0.84 \mathrm{fm}
$$

sendo $A$ o número de nucleons do núcleo. Devido a efeitos de estrutura nuclear específicos (partícula única e/ou coletivos), os parâmetros de raio e de difusividade mostram pequenas variações em torno dos correspondentes valores médios ao longo da tabela periódica (ver Figuras 3.1 e 3.2). Na Ref. [19], estas variações foram analisadas e os desvios padrões de $\sigma_{R}=0.07 \mathrm{fm}$ e $\sigma_{a}=0.025 \mathrm{fm}$, relativos às médias do raio e da difusividade, respectivamente, foram encontrados. 

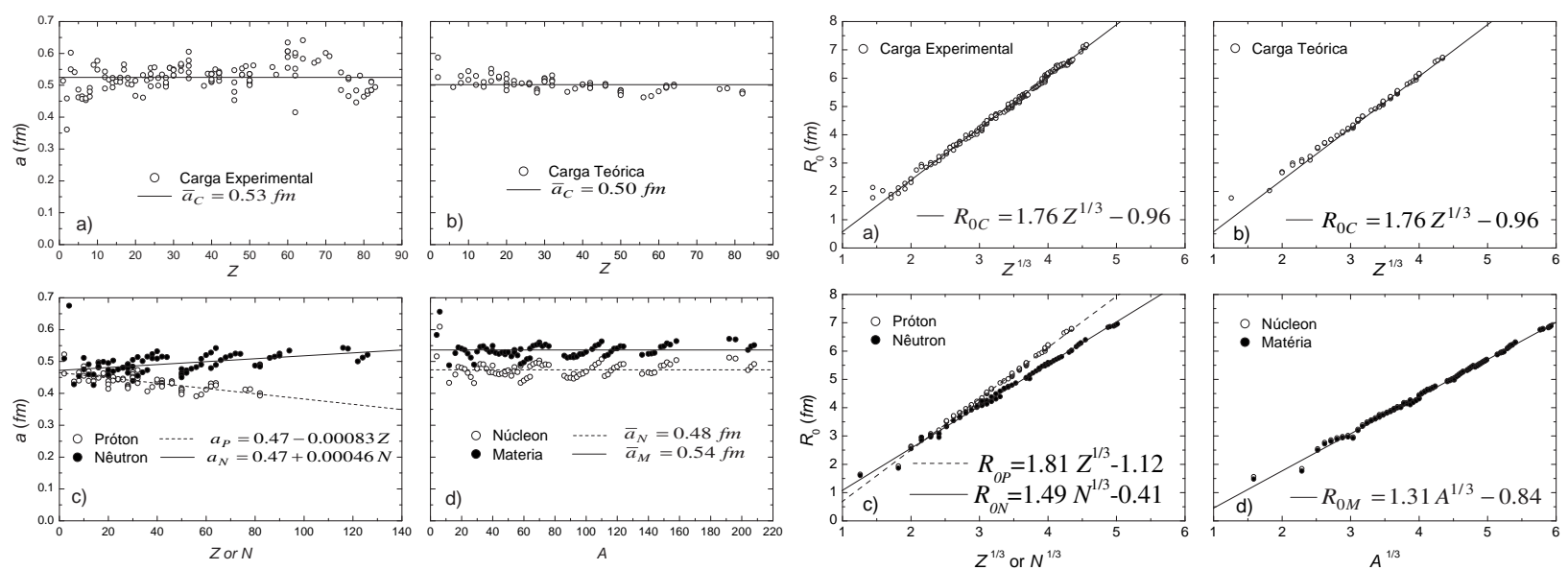

Figura 3.1: Valores equivalentes de difusividade a obtidos para distribuições de carga extraídos de experimentos de espalhamento de elétrons e para densidades teóricas obtidas de cálculos de Dirac-Hartree-Bogoliubov.

Figura 3.2: Valores do parâmetro de raio não deformado $R_{0}$ obtidos para distribuições de carga extraídos de experimentos de espalhamento de elétrons e para densidades teóricas obtidas de cálculos de Dirac-HartreeBogoliubov.

\subsection{Sistemática de Comprimentos de Deformação}

Na presente seção, com o objetivo de obter os parâmetros de deformação, usamos a sistemática para as probabilidades de transição de quadrupolo $B(E 2)$ e octupolo $B(E 3)$ de núcleos par-par das Refs. [50, 51]. Definimos os comprimentos de deformação correspondentes como $\delta_{2}=\beta_{2} R_{c} \mathrm{e}$ $\delta_{3}=\beta_{3} R_{c}$, e assumimos a seguinte conexão com as probabilidades de transição:

$$
B(E \lambda)=\left(\frac{3 Z e \beta_{\lambda} R_{c}^{\lambda}}{4 \pi}\right)^{2}
$$

A Figura 3.3 apresenta os valores extraídos para os comprimentos de deformação como uma função do número de prótons ou de nêutrons do núcleo. Fortes efeitos de estrutura são observados para o parâmetro $\delta_{2}$, que apresenta valores mínimos locais nos números mágicos. Com o intuito de enfatizar tal comportamento, calculamos valores médios sobre isótopos e também sobre isótonos (Figura 3.4(a)). Procedimento similar também foi aplicado ao parâmetro $\delta_{3}$ (Figura 3.4(b)). A maioria dos valores de $B(E 2)$ e $B(E 3)$ foram obtidos através de métodos que envolvem principalmente a interação eletromagnética, como excitação coulombiana, espalhamento de elétrons, etc. Logo, poderia-se esperar que os comprimentos de deformação extraídos deveriam relacionar-se principalmente com o 
número de prótons do núcleo. No entanto, uma inspeção das Figuras 3.3 e 3.4 mostra uma similaridade muito interessante entre o comportamento dos parâmetros de deformação como função de $Z \mathrm{e}$ $N$. Baseado nestas observações, propomos que os comprimentos de deformação podem ser aproximadamente descritos usando as seguintes funções:

$$
\begin{aligned}
& \delta_{2}=D_{2}(Z)+D_{2}(N), \\
& \delta_{3}=D_{3}(Z)+D_{3}(N),
\end{aligned}
$$

onde $D_{3}(X)=\frac{\alpha}{\sqrt{X}} \operatorname{com} \alpha=3.2 \mathrm{fm}$, e a função $D_{2}(X)$ é dada na Tabela 3.1. As expressões (3.8) e (3.9) descrevem o conjunto completo de comprimentos de deformação extraídos experimentalmente com uma dispersão (desvio padrão) de $0.2 \mathrm{fm}$ (em torno de 15\% de precisão). Esta precisão é somente um pouco superior que as incertezas experimentais.

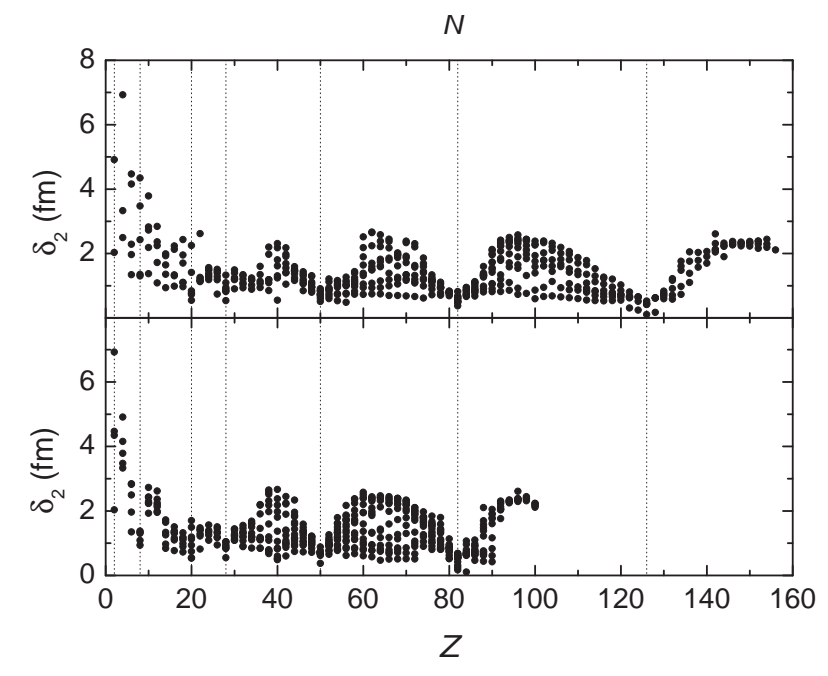

(a) Comprimentos de deformação quadrupolar.

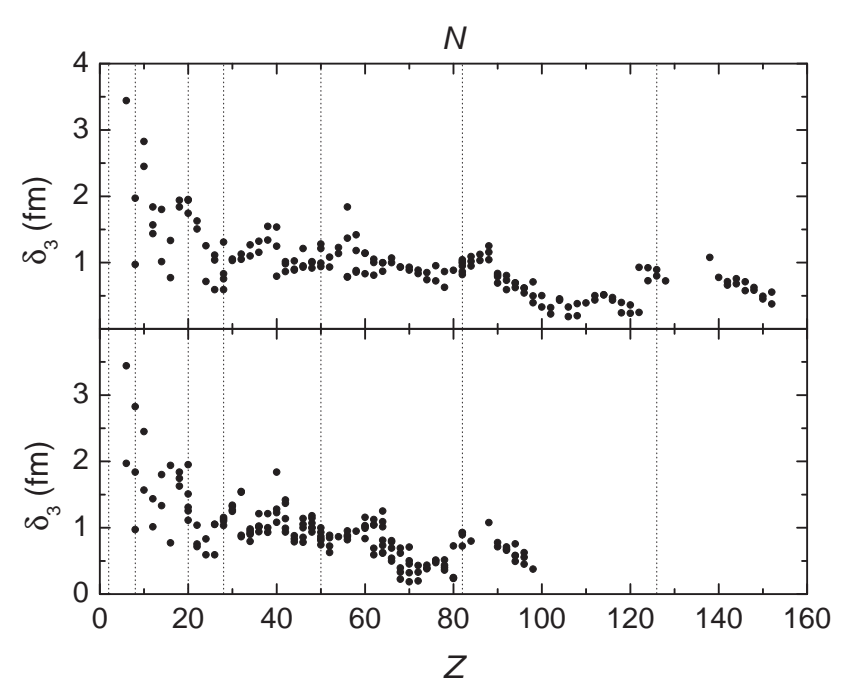

(b) Comprimentos de deformação octupolar.

Figura 3.3: Os valores dos comprimentos de deformação de quadrupolo (a) e de octupolo (b) como uma função do número de prótons (embaixo) ou nêutrons (em cima). As linhas tracejadas representam os números mágicos.

Uma comparação entre as funções $D_{2}$ e $D_{3}$ é mostrada na Figura 3.5. Os efeitos de estrutura de camada observados em $D_{2}$ mas não em $D_{3}$ são bem compreendidos microscopicamente. Em ordem mais baixa, transições coletivas de quadrupolo são construídas de combinações lineares de 


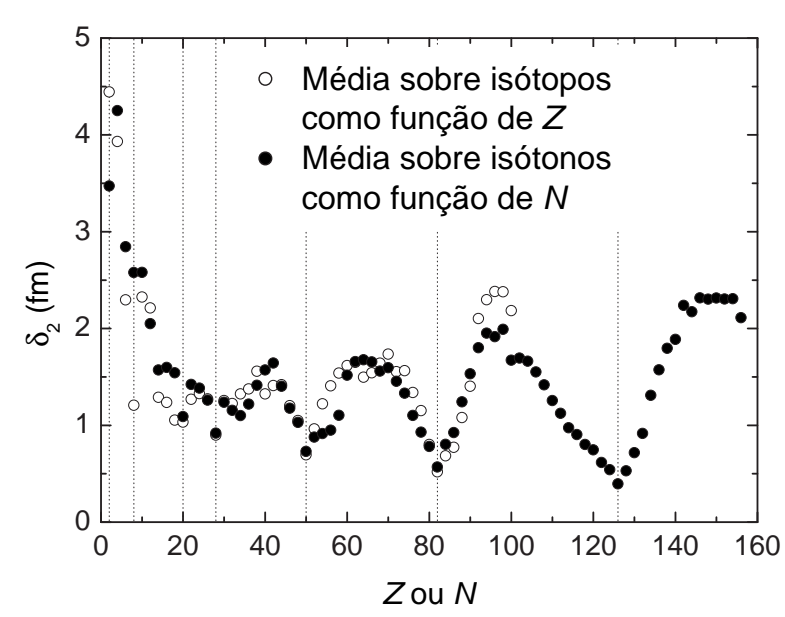

(a) Média dos comprimentos de deformação quadrupolar.

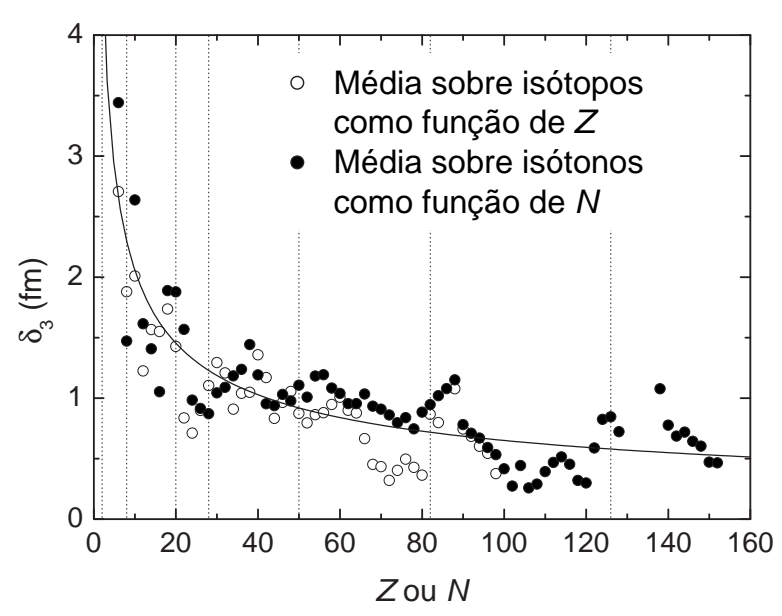

(b) Média dos comprimentos de deformação octupolar. A linha cheia representa uma função proporcional a $X^{-1 / 2}$, onde $X$ pode ser $Z$ ou $N$.

Figura 3.4: Os valores médios dos comprimentos de deformação de quadrupolo (a) e octupolo (b) para isótopos (pontos brancos) ou isótonos (pontos pretos) como função do número de prótons $Z$ (pontos brancos) ou de nêutrons $N$ (pontos pretos) do núcleo.

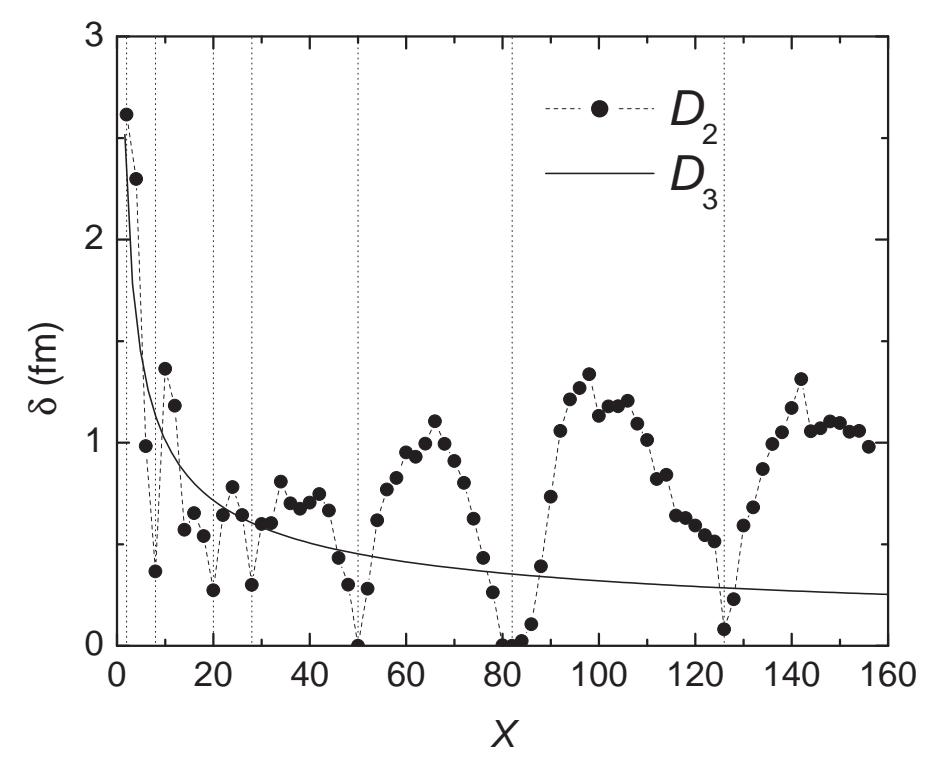

Figura 3.5: As funções $D_{2}$ e $D_{3}$ que descrevem o comportamento médio dos comprimentos de deformação. As linhas tracejadas representam os números mágicos.

excitações de partícula-buraco com $J=2$ e paridade positiva. No caso de camadas abertas, tais excitações envolvem estados da própria camada principal. No fechamento de camadas, porém, o 
Tabela 3.1: Valores da função $D_{2}(X)$

\begin{tabular}{cccccccc}
\hline$X$ & $D_{2}(\mathrm{fm})$ & $X$ & $D_{2}(\mathrm{fm})$ & $X$ & $D_{2}(\mathrm{fm})$ & $X$ & $D_{2}(\mathrm{fm})$ \\
\hline 2 & 2.61 & 42 & 0.75 & 82 & 0.00 & 122 & 0.54 \\
4 & 2.30 & 44 & 0.67 & 84 & 0.02 & 124 & 0.51 \\
6 & 0.98 & 46 & 0.43 & 86 & 0.11 & 126 & 0.08 \\
8 & 0.37 & 48 & 0.30 & 88 & 0.39 & 128 & 0.23 \\
10 & 1.36 & 50 & 0.00 & 90 & 0.73 & 130 & 0.59 \\
12 & 1.18 & 52 & 0.28 & 92 & 1.06 & 132 & 0.68 \\
14 & 0.57 & 54 & 0.62 & 94 & 1.21 & 134 & 0.87 \\
16 & 0.65 & 56 & 0.77 & 96 & 1.27 & 136 & 0.99 \\
18 & 0.54 & 58 & 0.83 & 98 & 1.34 & 138 & 1.05 \\
20 & 0.27 & 60 & 0.95 & 100 & 1.13 & 140 & 1.17 \\
22 & 0.64 & 62 & 0.93 & 102 & 1.18 & 142 & 1.31 \\
24 & 0.78 & 64 & 0.99 & 104 & 1.18 & 144 & 1.06 \\
26 & 0.64 & 66 & 1.10 & 106 & 1.21 & 146 & 1.07 \\
28 & 0.30 & 68 & 0.99 & 108 & 1.09 & 148 & 1.10 \\
30 & 0.60 & 70 & 0.91 & 110 & 1.01 & 150 & 1.10 \\
32 & 0.60 & 72 & 0.80 & 112 & 0.82 & 152 & 1.05 \\
34 & 0.81 & 74 & 0.63 & 114 & 0.84 & 154 & 1.06 \\
36 & 0.70 & 76 & 0.43 & 116 & 0.64 & 156 & 0.98 \\
38 & 0.67 & 78 & 0.26 & 118 & 0.63 & 158 & \\
40 & 0.70 & 80 & 0.00 & 120 & 0.59 & 160 & \\
\hline \hline
\end{tabular}

estado de partícula deve ser de duas camadas principais acima da camada fechada, o que inibe a excitação devido ao relativamente alto valor de energia envolvido na transição. $D_{2}$ é então grande onde uma camada está sendo preenchida e tende a zero quando ela se completa. Transições coletivas de octupolo, por outro lado, são uma combinação linear de excitações de partícula-buraco com $J=3$ e paridade negativa, que envolve um estado de buraco de uma camada e um estado de partícula da camada adjacente. Visto que tais combinações sempre existem, independentemente do fechamento de camadas, pouca estrutura é observada em $D_{3}$.

Esta sistemática para os comprimentos de deformação mostra características muito interessantes: (i) a simetria entre as contribuições de nêutrons e de prótons e (ii) o contraste entre os fortes efeitos de estrutura em $\delta_{2}$ comparados com o comportamento suave de $\delta_{3}$. A sistemática foi efetuada com base em resultados experimentais para núcleos estáveis par-par e a análise indica que pode ser usada para estimar os parâmetros de deformação dentro de uma precisão em torno de $15 \%$. Um teste das 
previsões para outros tipos de núcleos, como os exóticos, seria bastante interessante.

Nas próximas seções lidaremos com densidades deformadas assumindo a forma $2 \mathrm{pF}$ :

$$
\begin{gathered}
\rho(\vec{r})=\frac{\rho_{0}}{1+\exp \left(\frac{r-R\left(\theta_{0}\right)}{a}\right)}, \\
R\left(\theta_{0}\right)=R_{0}+\sum \delta_{\lambda} Y_{\lambda 0}\left(\theta_{0}\right),
\end{gathered}
$$

onde $\theta_{0}$ é o ângulo entre $\vec{r}$ e o eixo de simetria da deformação. Para distribuições de carga assumimos que $R_{0}$ pode ser descrito pela Eq. (3.5), e o estudo é limitado somente à deformações de quadrupolo e octupolo. As densidades não-deformadas correspondentes são referidas como:

$$
\rho^{(0)}(r)=\frac{\rho_{0}^{(0)}}{1+\exp \left(\frac{r-R_{0}}{a}\right)}
$$

Devido à condição de normalização, como definido pela Eq. (3.13), deve-se observar que $\rho_{0} \neq \rho_{0}^{(0)}$.

$$
\int \rho(\vec{r}) d \vec{r}=Z
$$

\subsection{Potencial Coulombiano}

A interação de Coulomb entre duas distribuições de carga é dada por:

$$
V_{C}(R)=\iint \frac{e^{2}}{\left|\vec{R}+\vec{r}_{2}-\vec{r}_{1}\right|} \rho_{1}\left(\vec{r}_{1}\right) \rho_{2}\left(\vec{r}_{2}\right) d \vec{r}_{1} d \vec{r}_{2}
$$

onde $\vec{R}$ é o vetor posição do centro de massa do núcleo 2 medido a partir do centro de massa do núcleo 1 (ver Figura 3.6) e descreve o movimento relativo entre eles. Assumiremos um sistema de coordenadas com o eixo $z$ na direção de $\vec{R}$ e o eixo $x$ de forma que o plano $x z$ contenha o eixo de simetria da deformação do núcleo 1 . Desta maneira a direção deste eixo de simetria é definida apenas pelo ângulo $\theta_{1}$. A direção do eixo de simetria da deformação do núcleo 2 é definida por 2 ângulos: $\theta_{2}$ e o ângulo azimutal $\phi_{2}$ (por conveniência, foi escolhido $\phi_{2}=0$ na Figura 3.6). 


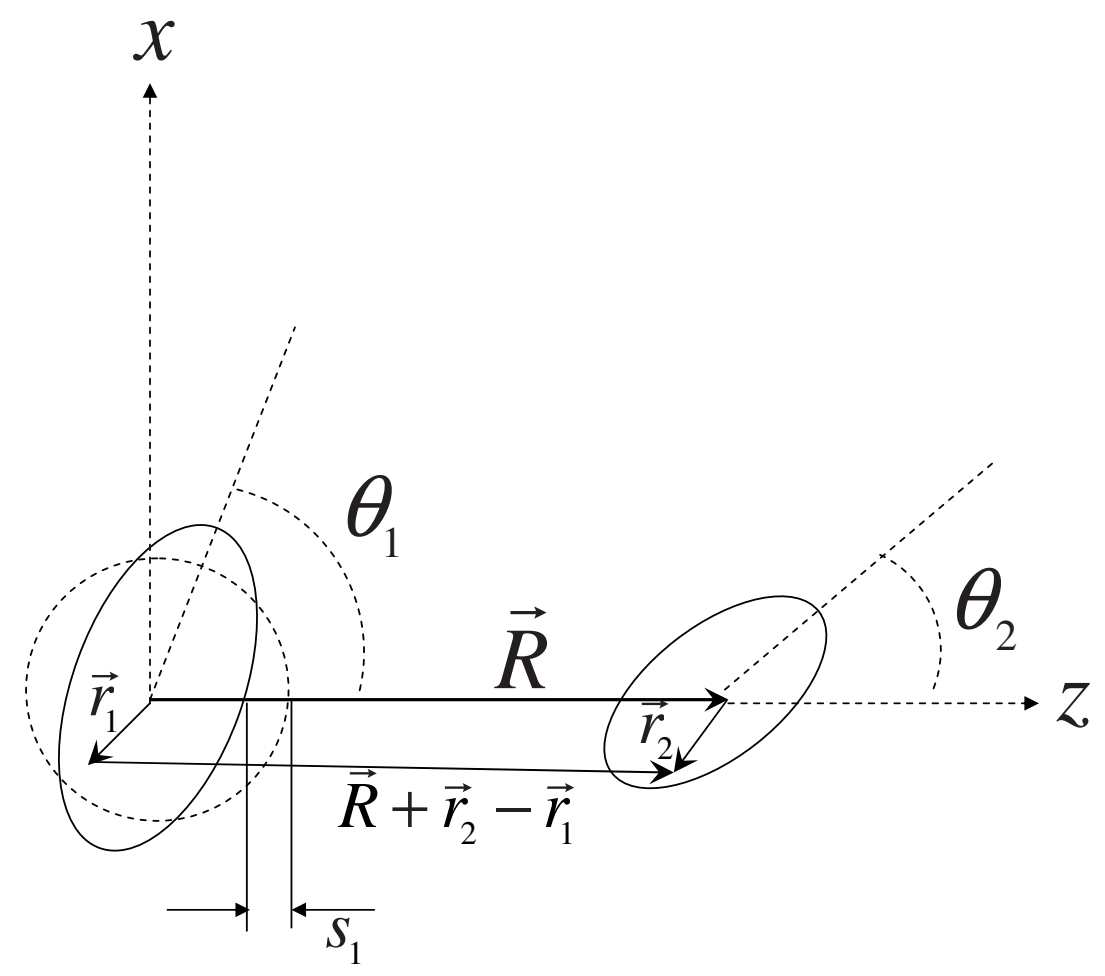

Figura 3.6: Figura esquemática da colisão de dois núcleos deformados. A circunferência tracejada representa a superfície do núcleo 1 se não houvesse deformação.

\subsubsection{Potencial Coulombiano Esférico}

Para densidades esfericamente simétricas, a representação da transformada de Fourier aplicada à Eq. (3.14) resulta em:

$$
\begin{gathered}
V_{C}^{(0)}(R)=\frac{2 Z_{1} Z_{2} e^{2}}{\pi} \int_{0}^{\infty} J_{0}(q R) \hat{\rho}_{1}(q) \hat{\rho}_{2}(q) d q \\
\hat{\rho}(q)=\frac{4 \pi}{Z} \int_{0}^{\infty} J_{0}(q r) r^{2} \rho^{(0)}(r) d r .
\end{gathered}
$$


Além disso, no caso de difusividade nula $(a=0)$ obtém-se:

$$
V_{C}^{(0)}(R)=\frac{18 Z_{1} Z_{2} e^{2}}{\pi} \int_{0}^{\infty} J_{0}(q R) \frac{J_{1}\left(q R_{01}\right)}{q R_{01}} \frac{J_{1}\left(q R_{02}\right)}{q R_{02}} d q
$$

A Figura 3.7 apresenta uma comparação entre os resultados para $V_{C}^{(0)}$ obtidos por: (i) Eq. (3.15) (usando a difusividade média da Ref. [19]: $a=0.53 \mathrm{fm}$ ), (ii) Eq. (3.17), i.e. $a=0$, (iii) Eq. (3.18) do modelo de carga pontual, e (iv) Eq. (3.19) do modelo de carga pontual mais distribuição uniforme de carga, que é freqüientemente assumida em cálculos de colisões de íons pesados. Os diferentes modelos fornecem resultados similares na região do raio da barreira e, conseqüentemente, tais modelos resultam em seções de choque similares exceto talvez em altas energias de bombardeio, onde a região interna da interação pode ser testada.

$$
\begin{gathered}
V_{C}^{(0)}(R)=\frac{Z_{1} Z_{2} e^{2}}{R} \\
V_{C}^{(0)}(R)=Z_{1} Z_{2} e^{2}\left\{\begin{array}{cc}
1 / R & \left(R>R_{C}=R_{01}+R_{02}\right) \\
\frac{1}{2 R_{C}}\left[3-\frac{R^{2}}{R_{C}^{2}}\right] & \left(R<R_{C}\right)
\end{array}\right.
\end{gathered}
$$

\subsubsection{Potencial Coulombiano Deformado}

Para densidades deformadas, a resolução computacional da Eq. (3.14) consome muito tempo graças à integral em seis dimensões. Isto se torna um problema em estudos onde ela deve ser resolvida diversas vezes como, por exemplo, em cálculos de fusão abaixo da barreira onde as seções de choque são obtidas como uma média sobre as diferentes orientações dos eixos de deformação. Logo, métodos mais rápidos para se obter boas aproximações para o potencial deformado se tornam bastante convenientes. Na Ref. [46] foi estudada a interação entre um projétil deformado e um alvo esfericamente simétrico, considerando ambas distribuições com $a=0$ (a maneira para estender os resultados para o caso de difusividades finitas foi indicado naquele artigo). Estes resultados serão usados como ponto de partida para apresentar expressões mais gerais.

Podemos dividir o potencial de Coulomb em duas contribuições: $V_{C}(R)=V_{C}^{(0)}(R)+V_{C}^{\text {Cor }}(R)$, 


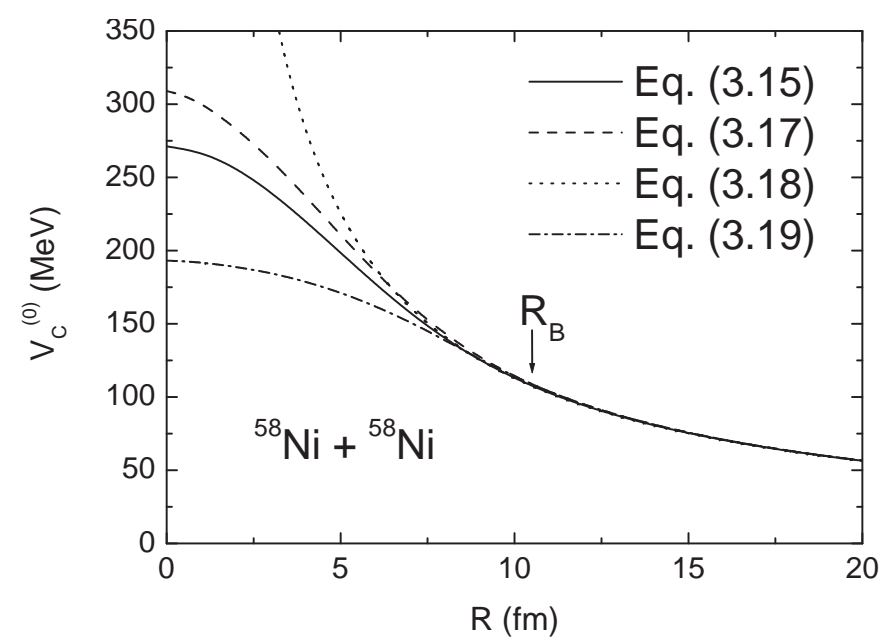

Figura 3.7: $O$ potencial coulombiano não-deformado para o sistema ${ }^{58} \mathrm{Ni}+{ }^{58} \mathrm{Ni}$ calculado através de diferentes modelos. A seta representa a posição aproximada do raio da barreira para a onda $\mathrm{s}$ correspondente.

onde $V_{C}^{(0)}(R)$ é obtido através da Eq. (3.15) usando a densidade não deformada correspondente. A Eq. (3.14) foi resolvida usando um método de Monte Carlo e, conseqüentemente, os resultados correspondentes possuem incertezas estatísticas. Neste estudo foi assumido o sistema ${ }^{58} \mathrm{Ni}+{ }^{58} \mathrm{Ni}$ como exemplo. Os parâmetros de deformação do ${ }^{58} \mathrm{Ni}$ são: $\beta_{2}=0.205 \mathrm{fm}$ e $\beta_{3}=0.235 \mathrm{fm}$, mas, em algumas simulações estes valores foram alterados, incluindo simulações onde os dois núcleos foram considerados como tendo deformações diferentes. Na Figura 3.8 é mostrado que $V_{C}^{\text {Cor é uma }}$ correção relativamente pequena de $V_{C}^{(0)}$ (em torno de $3 \%$ ) e, conseqüentemente, a incerteza estatística no cálculo de $V_{C}$, através da Eq. (3.14), deve ser muito pequena para fornecer resultados úteis para $V_{C}^{\text {Cor }}$. Porém, apesar de relativamente pequena, a correção é importante pois pode atingir até cerca de $4 \mathrm{MeV}$ na região do raio da barreira (ver Figura 3.8) e isto certamente produz efeitos grandes em, por exemplo, seções de choque de fusão.

De acordo com a Ref. [46], se apenas o núcleo 1 é deformado, os eixos de simetria das deformações estão na mesma direção e a difusividade das distribuições é nula $(a=0)$, a correção do potencial coulombiano pode ser aproximadamente descrita pelo seguinte conjunto de equações:

$$
\begin{gathered}
V_{C}^{\mathrm{Cor}}(R)=V^{(1)}(R)+V^{(2)}(R) \\
V^{(1)}(R)=Z_{1} Z_{2} e^{2}\left[\beta_{21} F_{2}^{(1)}\left(R ; R_{01} ; R_{02}\right) Y_{20}\left(\theta_{1}\right)+\beta_{31} F_{3}^{(1)}\left(R ; R_{01} ; R_{02}\right) Y_{30}\left(\theta_{1}\right)\right]
\end{gathered}
$$




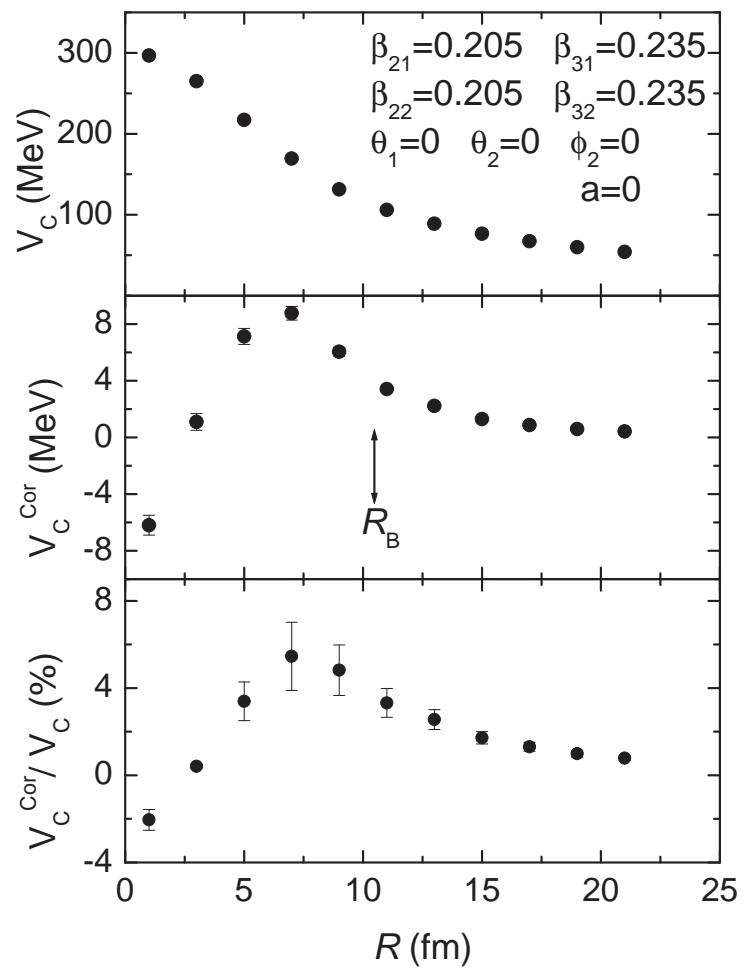

Figura 3.8: A figura apresenta o potencial coulombiano total $\left(V_{C}\right)$, a correção $\left(V_{C}^{\mathrm{Cor}}\right)$ e os valores relativos correspondentes $\left(100 V_{C}^{\text {Cor }} / V_{C}\right)$ para o sistema ${ }^{58} \mathrm{Ni}+{ }^{58} \mathrm{Ni}$. A seta indica a posição aproximada do raio da barreira.

$$
\begin{aligned}
V^{(2)}(R)= & \frac{Z_{1} Z_{2} e^{2}}{\sqrt{\pi}}\left[\beta_{21}^{2} G_{2}\left(R ; R_{01} ; R_{02} ; \theta_{1}\right)\right. \\
& \left.+\beta_{31}^{2} G_{3}\left(R ; R_{01} ; R_{02} ; \theta_{1}\right)+\beta_{21} \beta_{31} G_{23}\left(R ; R_{01} ; R_{02} ; \theta_{1}\right)\right] \\
G_{2}\left(R ; X ; Y ; \theta_{1}\right)= & \sqrt{\frac{5}{49}} F_{2}^{(2)}(R ; X ; Y) Y_{20}\left(\theta_{1}\right)+\frac{3}{7} F_{4}^{(2)}(R ; X ; Y) Y_{40}\left(\theta_{1}\right) \\
G_{3}\left(R ; X ; Y ; \theta_{1}\right)= & \sqrt{\frac{4}{45}} F_{2}^{(2)}(R ; X ; Y) Y_{20}\left(\theta_{1}\right)+\frac{3}{11} F_{4}^{(2)}(R ; X ; Y) Y_{40}\left(\theta_{1}\right) \\
& +\sqrt{\frac{2500}{14157}} F_{6}^{(2)}(R ; X ; Y) Y_{60}\left(\theta_{1}\right)
\end{aligned}
$$




$$
\begin{aligned}
& G_{23}\left(R ; X ; Y ; \theta_{1}\right)= \sqrt{\frac{27}{35}} F_{1}^{(2)}(R ; X ; Y) Y_{10}\left(\theta_{1}\right)+\sqrt{\frac{16}{45}} F_{3}^{(2)}(R ; X ; Y) Y_{30}\left(\theta_{1}\right) \\
&+\sqrt{\frac{500}{693}} F_{5}^{(2)}(R ; X ; Y) Y_{50}\left(\theta_{1}\right) \\
& F_{\lambda}^{(1)}(R ; X ; Y)=\frac{18}{\pi} \int_{0}^{\infty} J_{\lambda}(q R) \frac{J_{1}(q Y)}{q Y} J_{\lambda}(q X) d q, \\
& F_{\lambda}^{(2)}(R ; X ; Y)=\frac{18}{\pi} \int_{0}^{\infty} J_{\lambda}(q R) \frac{J_{1}(q Y)}{q Y}\left[J_{\lambda}(q X)+\frac{q X}{2} \frac{\mathrm{d} J_{\lambda}(q X)}{\mathrm{d}(q X)}\right] d q
\end{aligned}
$$

A partir deste conjunto de equações e desprezando termos de segunda ordem pode-se encontrar a seguinte expressão assintótica:

$$
V_{C}^{\text {Cor }}(R \rightarrow \infty)=Z_{1} Z_{2} e^{2}\left[\frac{3 \beta_{21} R_{01}^{2}}{5 R^{3}} Y_{20}\left(\theta_{1}\right)+\frac{3 \beta_{31} R_{01}^{3}}{7 R^{4}} Y_{30}\left(\theta_{1}\right)\right]
$$

Esta expressão (ou similares) tem sido usada em diversos trabalhos (e.g. Ref. [12]).

As linhas cheias na Figura 3.9 representam os resultados das Eqs. (3.20) a (3.27) e as linhas tracejadas correspondem à Eq. (3.28). Os "pontos experimentais” na figura correspondem, na verdade, a simulações de Monte Carlo para a resolução da integral em seis dimensões representada pela Eq. (3.14). As Eqs. (3.20) a (3.27) fornecem resultados muito bons, exceto a distâncias muito internas. Deve-se observar que a expressão assintótica não é acurada sob certas condições. De fato, no caso da Figura 3.9(c), por exemplo, os termos de segunda ordem não são de forma alguma desprezíveis.

Generalizamos [38] o modelo para dois núcleos deformados e considerando diferentes orientações $\left(\theta_{\lambda i}, \phi_{\lambda i}\right)$ para cada deformação de cada núcleo. A correção correspondente $\left(V_{C}^{\text {Cor }}\right)$ pode ser descrita pelas seguintes modificações para $V^{(1)}$ e $V^{(2)}$ :

$$
\begin{aligned}
V^{(1)}(R)= & Z_{1} Z_{2} e^{2}\left[\beta_{21} F_{2}^{(1)}\left(R ; R_{01} ; R_{02}\right) Y_{20}\left(\theta_{21}\right)+\beta_{31} F_{3}^{(1)}\left(R ; R_{01} ; R_{02}\right) Y_{30}\left(\theta_{31}\right)\right. \\
& \left.+\beta_{22} F_{2}^{(1)}\left(R ; R_{02} ; R_{01}\right) Y_{20}\left(\theta_{22}\right)-\beta_{32} F_{3}^{(1)}\left(R ; R_{02} ; R_{01}\right) Y_{30}\left(\theta_{32}\right)\right]
\end{aligned}
$$




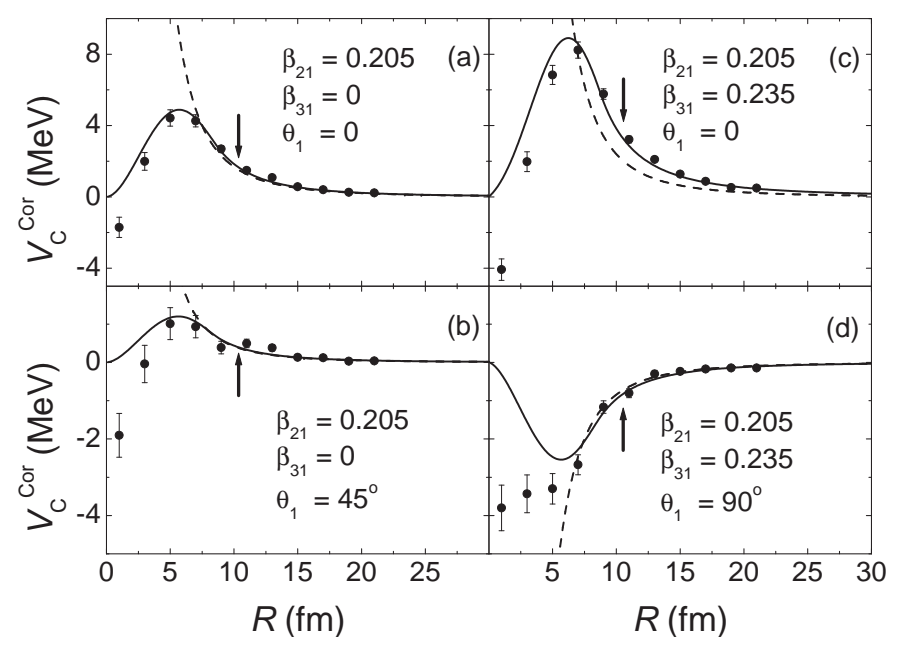

Figura 3.9: A figura apresenta a correção do potencial coulombiano para o sistema ${ }^{58} \mathrm{Ni}+{ }^{58} \mathrm{Ni}$. $O$ núcleo 2 é assumido como não-deformado e a difusividade de ambas distribuições é $a=0$. Os parâmetros de deformação do núcleo são indicados na figura. Os "pontos experimentais" na figura correspondem, na verdade, a simulações de Monte Carlo para a resolução da integral em seis dimensões representada pela Eq. (3.14). As linhas cheias representam os resultados das Eqs. (3.20) - (3.27) e as linhas tracejadas representam a expressão assintótica (3.28). As setas indicam a posição aproximada do raio da barreira.

$$
\begin{aligned}
V^{(2)}(R)= & Z_{1} Z_{2} e^{2}\left\{\sum_{\substack{\lambda \neq 0 \\
\lambda_{a} \lambda_{b}}}(2 \lambda+1)\left(\begin{array}{ccc}
\lambda_{a} & \lambda_{b} & \lambda \\
0 & 0 & 0
\end{array}\right)\right. \\
& \times\left[F_{\lambda}^{(2)}\left(R ; R_{01} ; R_{02}\right) \beta_{\lambda_{a} 1} \beta_{\lambda_{b} 1} \times H_{\lambda_{\lambda_{a} \lambda_{b}}}\left(\theta_{\lambda_{a} 1}, \phi_{\lambda_{a} 1} ; \theta_{\lambda_{b} 1}, \phi_{\lambda_{b} 1}\right)\right. \\
& +(-1)^{\lambda} F_{\lambda}^{(2)}\left(R ; R_{02} ; R_{01}\right) \beta_{\lambda_{a} 2} \beta_{\lambda_{b} 2} \times H_{\lambda \lambda_{a} \lambda_{b}}\left(\theta_{\lambda_{a} 2}, \phi_{\lambda_{a} 2} ; \theta_{\lambda_{b} 2}, \phi_{\lambda_{b} 2}\right) \\
& \left.\left.+i^{\lambda+\lambda_{b}-\lambda_{a}} F_{\lambda \lambda_{a} \lambda_{b}}^{(3)}\left(R ; R_{01} ; R_{02}\right) \beta_{\lambda_{a} 1} \beta_{\lambda_{b} 2} \times H_{\lambda_{\lambda_{a} \lambda_{b}}}\left(\theta_{\lambda_{a} 1}, \phi_{\lambda_{a} 1} ; \theta_{\lambda_{b} 2}, \phi_{\lambda_{b} 2}\right)\right]\right\} \\
& F_{\lambda \lambda_{a} \lambda_{b}}^{(3)}(R ; X ; Y)=\frac{18}{\pi} \int_{0}^{\infty} J_{\lambda}(q R) J_{\lambda_{a}}(q X) J_{\lambda_{b}}(q Y) d q \\
& H_{\lambda \lambda_{a} \lambda_{b}}\left(\theta_{1}, \phi_{1} ; \theta_{2}, \phi_{2}\right)=\sum_{\mu_{a} \mu_{b}}\left(\begin{array}{ccc}
\lambda_{a} & \lambda_{b} & \lambda \\
\mu_{a} & \mu_{b} & 0
\end{array}\right) Y_{\lambda_{a} \mu_{a}}^{*}\left(\theta_{1}, \phi_{1}\right) \times Y_{\lambda_{b} \mu_{b}}^{*}\left(\theta_{2}, \phi_{2}\right)
\end{aligned}
$$

onde foram usados os símbolos 3- $j$ [52].

Nossas simulações indicaram que tais expressões são bastante acuradas (Figura 3.10(a)). Finalmente, para completar a generalização, observamos (Figura 3.10(b)) que o modelo também funciona 
para distribuições com $a \neq 0$, usando as mesmas expressões (com $a=0$ ) para $V_{C}^{\text {Cor }}$, mas considerando no cálculo de $V_{C}^{(0)}$, através das Eqs. (3.15) e (3.16), as densidades não deformadas correspondentes com $a \neq 0$. Este ponto é importante pois $V_{C}^{(0)}$ tem dependência em $a$. Na Figura 3.10 pode-se observar, novamente, que a expressão assintótica dada pela Eq. (3.28), generalizada para dois núcleos deformados, falha em alguns casos.

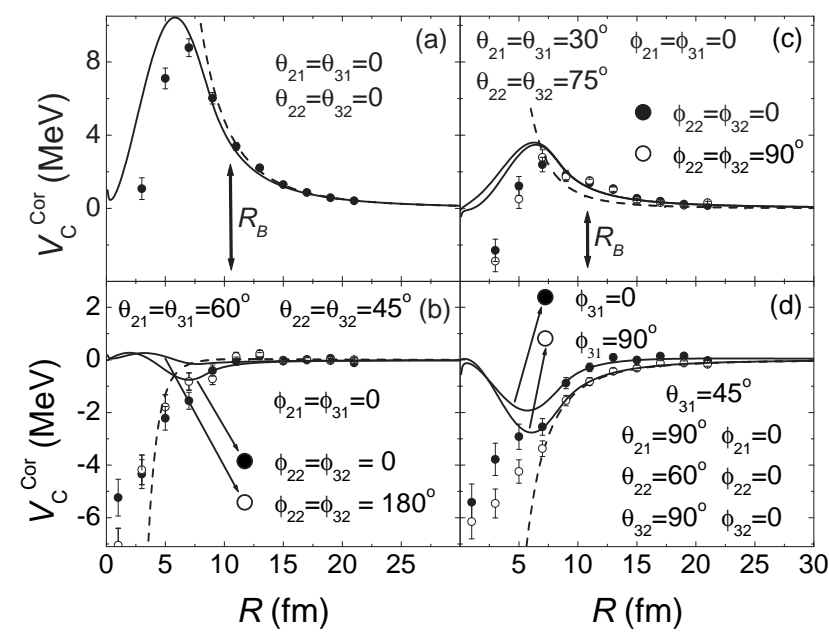

(a) $a=0$ assumido para ambos os núcleos.

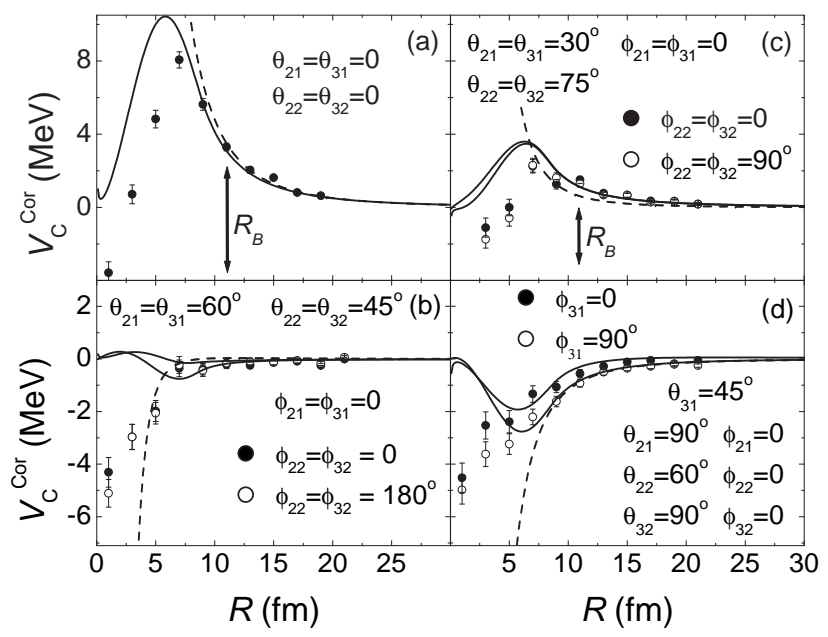

(b) $a=0.53 \mathrm{fm}$ assumido para ambos os núcleos.

Figura 3.10: As figuras apresentam as correções do potencial coulombiano para o sistema ${ }^{58} \mathrm{Ni}+$ ${ }^{58} \mathrm{Ni}$, onde $\beta_{2}=0.205, \beta_{3}=0.235$. Os ângulos dos eixos de deformação são indicados nas figuras. Os "pontos experimentais" na figura correspondem, na verdade, a simulações de Monte Carlo para a resolução da integral em seis dimensões representada pela Eq. (3.14). As linhas cheias representam os resultados das Eqs. (3.29) - (3.32) e as linhas tracejadas representam a expressão assintótica (3.28). As setas indicam a posição aproximada do raio da barreira.

Nesta seção foi fornecido um modelo para calcular o potencial coulombiano entre dois núcleos deformados com difusividades finitas. Este modelo produz estimativas precisas na região da superfície, onde as expressões assintóticas usuais falham em certos casos. Uma estimativa razoável da interação coulombiana deformada também é obtida na região interna. Na Ref. [53] pode ser encontrada uma maneira ainda mais acurada de se obter o potencial coulombiano deformado. 


\subsection{Potencial de São Paulo}

Nosso grupo de pesquisa vem desenvolvendo um modelo para a interação nuclear, o Potencial de São Paulo (SP), que é baseado em efeitos da não-localidade de Pauli [17-19]. Esta interação demonstrou ter sucesso em descrever espalhamento elástico, canais de reação periféricos e fusão envolvendo muitos sistemas de íons pesados em uma ampla região de energia [22, 24-29, 54, 55]. No modelo não-local, a interação nuclear $V_{N}$ é conectada com o potencial de convolução (folding) através de [19]:

$$
V_{N}(R)=V_{F}(R) e^{-4 v^{2} / c^{2}}
$$

onde $c$ é a velocidade da luz no vácuo, $V_{F}$ é o potencial de convolução ${ }^{2}, v$ é a velocidade relativa local entre os dois núcleos:

$$
\mathrm{v}^{2}(R, E)=\frac{2}{\mu}\left[E-V_{C}(R)-V_{N}(R, E)\right],
$$

e $V_{C}$ é o potencial coulombiano. O potencial de convolução, por sua vez, é dado, em termos das densidades de nucleons e da interação nucleon-nucleon, por:

$$
V_{F}(R)=\int \rho_{N 1}\left(\overrightarrow{r_{1}}\right) \rho_{N 2}\left(\overrightarrow{r_{2}}\right) v_{N N}\left(\vec{R}-\overrightarrow{r_{1}}+\overrightarrow{r_{2}}\right) d \overrightarrow{r_{1}} d \overrightarrow{r_{2}}
$$

\subsubsection{Potencial de São Paulo Esférico}

Usualmente, na Eq. (3.35), $\rho_{N i}$ são as densidades de nucleons dos núcleos e $v_{N N}$ é a interação efetiva nucleon-nucleon. No modelo de São Paulo, assumimos a seguinte interação nucleon-nucleon:

$$
v_{N N}(r)=\int \rho_{m}\left(r_{1}\right) \rho_{m}\left(r_{2}\right) V_{0} \delta\left(\vec{r}-\overrightarrow{r_{1}}+\overrightarrow{r_{2}}\right) d \overrightarrow{r_{1}} d \overrightarrow{r_{2}}
$$

onde $V_{0}=-456 \mathrm{MeV} \mathrm{fm}^{3}$ e $\rho_{m}$ é a densidade de matéria do nucleon. Baseado na distribuição intrínseca de carga do próton no espaço livre, que é determinada por experimentos de espalhamento de elétrons, uma forma exponencial foi assumida para a densidade de matéria do nucleon [19]. Devido à função delta, a convolução na Eq. (3.36) é chamada de aproximação de alcance zero (zero-range

\footnotetext{
${ }^{2} \mathrm{O}$ potencial de convolução foi identificado por $V_{F}$, em alusão ao termo em inglês folding que é também bastante empregado, para que não haja confusão com $V_{C}$ que se refere ao potencial coulombiano.
} 
approach). Como foi mostrado na Seção 3.1, especificamente na Eq. (3.4) (página 27), também podemos definir [19] a densidade de matéria do núcleo ao fazer a convolução da densidade de nucleon correspondente com a densidade intrínseca de matéria do nucleon. Logo, distinguimos a densidade de matéria do núcleo da distribuição de nucleons do núcleo, levando em conta o tamanho finito do nucleon. Inserindo as Eqs. (3.36) e (3.4) na Eq. (3.35), o potencial de convolução pode ser reescrito da seguinte forma:

$$
\begin{aligned}
V_{F}(R) & =\int \rho_{M 1}\left(\overrightarrow{r_{1}}\right) \rho_{M 2}\left(\overrightarrow{r_{2}}\right) V_{0} \delta\left(\vec{R}-\overrightarrow{r_{1}}+\overrightarrow{r_{2}}\right) d \overrightarrow{r_{1}} d \overrightarrow{r_{2}} \\
& =V_{0} \int \rho_{M 1}\left(\overrightarrow{r_{1}}\right) \rho_{M 2}\left(\overrightarrow{r_{1}}-\vec{R}\right) d \overrightarrow{r_{1}}
\end{aligned}
$$

O uso das densidades de matéria e da função delta na Eq. (3.37) corresponde à aproximação de alcance zero para o potencial de convolução, que é totalmente equivalente ao procedimento mais usual de usar uma interação nucleon-nucleon efetiva com as densidades de nucleon do núcleo (ao invés das densidades de matéria), como mostra a Eq. (3.35). Esta equivalência matemática está relacionada ao vínculo estabelecido pela Eq. (3.4) (página 27). Entretanto, em nossos cálculos teóricos, utilizaremos a sistemática de densidades, descrita na Seção 3.1. Como vimos, dentro do contexto desta sistemática, tanto a densidade de matéria como a distribuição de nucleons são associadas à distribuições do tipo 2 pF. Assim sendo, a relação expressa na Eq. (3.4) não é obedecida de forma exata pelas distribuições da sistemática. Mesmo assim, as aproximações de alcance zero e alcance finito resultam em potenciais muito semelhantes. De forma a ilustrar este ponto, o qual é extensivamente discutido nas Refs. [19, 38], na Figura 3.11 mostramos o potencial de SP não-deformado calculado através da aproximação de alcance zero (linhas tracejadas) e através de cálculo de alcance finito (linhas cheias), para quatro sistemas em energias iguais às alturas das barreiras coulombianas, $E=V_{B}$. A diferença entre as linhas cheias e tracejadas é quase indistinguível na figura, indicando que as duas abordagens são de fato equivalentes. No entanto, devido à integral em seis dimensões, o método de alcance finito consome muito mais tempo computacional em cálculos numéricos de potenciais deformados do que a aproximação de alcance zero [38]. Logo, em todo o presente trabalho, especificamente nos cálculos mostrados nos Capítulos 4, 5, 6 e 7, assumimos a aproximação de alcance zero em nossos cálculos teóricos de seções de choque de fusão e de espalhamento. Estudaremos, então, neste capítulo, apenas 
o potencial de convolução dado pela Eq. (3.37), visto que interação nuclear correspondente pode ser obtida através da Eq. (3.33), considerando o termo envolvendo a velocidade relativa local, Eq. (3.34).

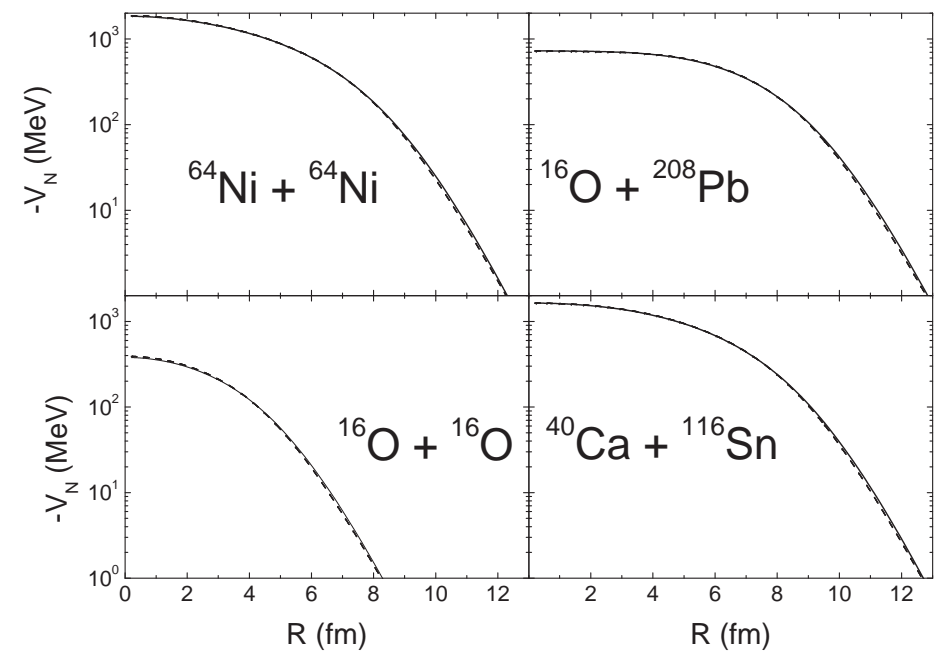

Figura 3.11: O potencial de São Paulo nas abordagens de alcance zero (linhas tracejadas) e finito (linhas cheias) para quatro sistemas indicados na figura.

Na Ref. [19], é fornecida também uma sistemática para as densidades de nucleon e de matéria. Os raios de ambas as distribuições são bem descritos por:

$$
R_{0}=1.31 A^{1 / 3}-0.84 \mathrm{fm}
$$

Devido ao procedimento de convolução, a difusividade média para as densidades de matéria, $a_{M}=$ $0.56 \mathrm{fm}$, é ligeiramente superior que aquela encontrada para a distribuição de nucleons, $a_{N}=0.50$ fm.

\subsubsection{Potencial de São Paulo Deformado}

A resolução numérica da Eq. (3.35) implica em um problema similar ao encontrado no estudo do potencial coulombiano na Seção. 3.3, devido à integral em seis dimensões. A Eq. (3.37) é de muito mais fácil resolução porque a aproximação de alcance zero reduz a integral original, Eq. (3.35), a uma integral tridimensional, produzindo praticamente o mesmo resultado (ver Figura 3.11). O potencial de convolução foi calculado, através da Eq. (3.37), para o sistema ${ }^{58} \mathrm{Ni}+{ }^{58} \mathrm{Ni}$ (linhas cheias na Figura 
3.12). Com intuito de comparação, as linhas tracejadas na Figura 3.12 representam o potencial de convolução não-deformado $\left(V_{F}^{(0)}\right)$, que foi calculado considerando as densidades não deformadas correspondentes. Na superfície, o potencial deformado pode diferir de um fator aproximadamente 4 em comparação com o não-deformado.

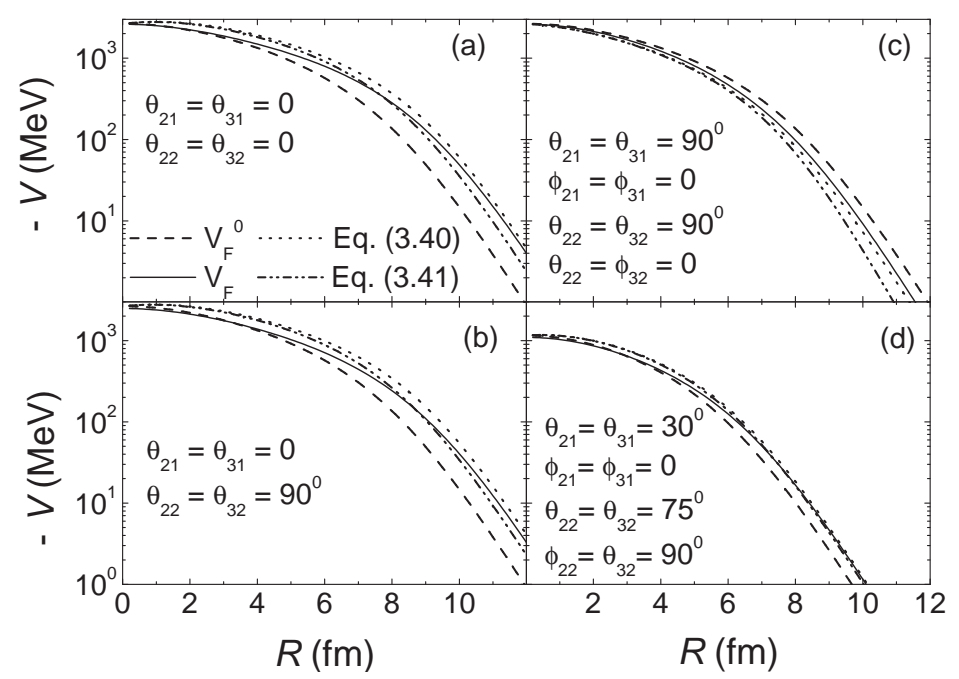

Figura 3.12: O potencial nuclear deformado (linhas cheias) é comparado com o não-deformado (linhas tracejadas) para o sistema ${ }^{58} \mathrm{Ni}+{ }^{58} \mathrm{Ni}$. A figura apresenta também os resultados obtidos com as aproximações usuais, Eqs. (3.40) $e$ (3.41). $\beta_{2}=0.205, \beta_{3}=0.235$ e $a_{M}=0.56$ fm foram assumidos para ambos os núcleos. Os ângulos dos eixos de deformação são indicados na figura.

Para grandes distâncias, o potencial não-deformado tem uma forma aproximada de uma exponencial

$$
V_{F}^{0}(R) \approx U_{0} e^{-\left(R-R_{01}-R_{02}\right) / a_{P}}
$$

onde o índice $P$ foi usado para diferenciar a difusividade do potencial da difusividade da densidade. Levando em conta que $V_{F}^{(0)}$ depende principalmente da distância entre as superfícies dos núcleos ( $s=R-R_{01}-R_{02}$ ), foram propostas expressões aproximadas para descrever o potencial deformado. Por exemplo, Eqs. (3.40) e (3.41) dependem da variação dos raios das densidades ao longo do eixo $z$ (A Figura 3.6, na página 33 mostra $s$ apenas para o núcleo 1).

$$
\begin{gathered}
V_{F}(R) \approx V_{F}^{(0)}(R) e^{s / a_{P}}, \\
V_{F}(R) \approx V_{F}^{(0)}(R)-s \frac{d V_{F}^{(0)}}{d R},
\end{gathered}
$$


onde a variação dos raios é definida por:

$$
s=\delta_{21} Y_{20}\left(\theta_{21}\right)+\delta_{31} Y_{30}\left(\theta_{31}\right)+\delta_{22} Y_{20}\left(\theta_{22}\right)-\delta_{32} Y_{30}\left(\theta_{32}\right)
$$

Levando em conta a forma aproximadamente exponencial do potencial, as difusividades envolvidas na Eq. (3.40) podem ser estimadas como:

$$
a_{P}=-V_{F}^{(0)}(R)\left[\frac{d V_{F}^{(0)}}{d R}\right]^{-1}
$$

A Figura 3.12 mostra os resultados para os potenciais folding deformados e não deformados e também os obtidos pelas Eqs. (3.40) e (3.41). Na Figura 3.13, mostramos as correções correspondentes $V_{F}^{C o r}=V_{F}-V_{F}^{(0)}$. Eq. (3.40) fornece melhores aproximações que a Eq. (3.41). Mesmo assim, na região da superfície a correção obtida a partir da Eq. (3.40) pode diferir em torno de $40 \%$ de resultados mais acurados obtidos com a Eq. (3.37).

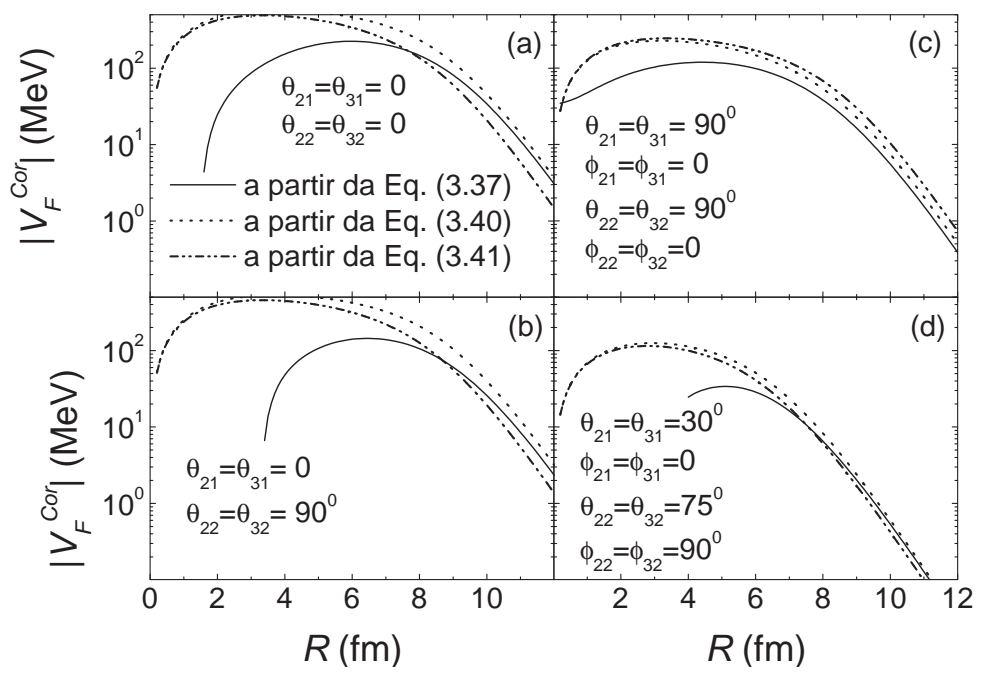

Figura 3.13: $O$ valor absoluto da correção $V_{F}^{C o r}=V_{F}-V_{F}^{(0)}$, onde o potencial de convolução foi obtido a partir das Eqs. (3.37), (3.40) e (3.41). Os parâmetros de deformação são os mesmos daqueles da Figura 3.12.

A maneira aqui proposta para calcular o potencial nuclear, que é baseada na aproximação de alcance zero (zero-range approach), torna os cálculos numéricos muito mais velozes do que se a interação efetiva nucleon-nucleon de alcance finito for usada. Foi demonstrado que as aproximações 
usuais podem diferir significativamente de resultados mais acurados. 


\section{Capítulo 4}

\section{Fusão Núcleo-Núcleo $\left({ }^{64} \mathbf{N i}+{ }^{64} \mathbf{N i}\right)$}

Neste capítulo usamos os diferentes modelos propostos no Capítulo 2 para descrever o processo de fusão para o sistema ${ }^{64} \mathrm{Ni}+{ }^{64} \mathrm{Ni}$ (dados obtidos das Refs. $\left.[8,56,57]\right)$ e comparamos seus correspondentes resultados. Escolhemos este sistema como exemplo pois seus dados de fusão apresentam enhancements muito grandes em comparação com os resultados dos cálculos do modelo de penetração de barreira não-deformada (BPM).

Ao lidar com um sistema complexo de muitas partículas, como um núcleo, acoplamentos aos graus de liberdade internos podem afetar significativamente a transmissão, principalmente em energias abaixo da barreira (e.g. [58-61]). Para as colisões núcleo-núcleo, os graus de liberdade internos podem representar deformações estáticas ou vibrações coletivas de superfície, correspondendo a excitações inelásticas, ou mesmo outros processos como fusão induzida por canais de transferência. Para excitações inelásticas, no contexto da Aproximação Congelada (FA), descrita em detalhes na Seção 2.2, o coeficiente de transmissão, relacionado à seção de choque de fusão, pode ser obtido realizando uma média sobre diferentes configurações do sistema. Este procedimento é equivalente ao cálculo de Canais Acoplados (CC, detalhes na Seção 2.1) apenas para energias de excitação desprezíveis. No presente capítulo comparamos os resultados obtidos pelo modelo da Aproximação Congelada Generalizada (GFA) que propusemos na Seção 2.3 como uma generalização da FA para energias de excitação diferente de zero. Comparamos também os resultados obtidos pelo modelo que propusemos na Seção 2.4, o modelo de Movimento de Ponto Zero (ZPM), que se trata de uma generalização do trabalho descrito na Ref. [14]. 
Inicialmente, porém, descreveremos o método utilizado para a obtenção dos parâmetros associados ao potencial efetivo, como a curvatura da barreira, e os fatores de forma dos modos excitação de quadrupolo e octupolo do ${ }^{64} \mathrm{Ni}$. A interação bare assumida em nossos cálculos foi o potencial de São Paulo, descrito em maiores detalhes no Capítulo 3, em especial na Seção 3.4.

\subsection{Potencial Efetivo e Fatores de Forma}

No contexto do BPM, o potencial efetivo é uma soma das partes coulombiana, nuclear e centrífuga:

$$
V_{\mathrm{ef}}(R)=V_{C}(R)+V_{N}(R)+\frac{\ell(\ell+1) \hbar^{2}}{2 \mu R^{2}} .
$$

A seção de choque do BPM é associada com o fluxo transmitido através da expressão:

$$
\sigma_{\mathrm{BPM}}(E)=\frac{\pi}{k^{2}} \sum(2 \ell+1) T_{\ell}
$$

Em nossos cálculos, a soma na Eq. (4.2) é efetuada até uma onda parcial máxima $\ell_{\max }$, que é o maior valor de $\ell$ que resulta em um poço interno (e uma barreira) no correspondente potencial efetivo. Para ondas $\ell$ com altura efetiva de barreira $\left(V_{B \ell}\right)$ abaixo da energia do centro de massa, aproximamos o potencial efetivo por uma parábola com curvatura:

$$
\hbar \omega_{\ell}=\left|\frac{\hbar^{2}}{\mu} \frac{\mathrm{d}^{2} V_{\mathrm{ef}}}{\mathrm{d} R^{2}}\right|_{R_{B \ell}}^{1 / 2},
$$

onde $R_{B \ell}$ é o raio da barreira. Por outro lado, para ondas $\ell$ com $V_{B \ell} \gg E$, a aproximação do potencial efetivo por uma barreira parabólica não é apropriada devido ao longo alcance do potencial coulombiano, e o cálculo dos coeficientes de transmissão através da expressão de Hill-Wheeler, Eq. (2.13), usando a curvatura fornecida pela Eq. (4.3) não é mais acurada. Logo, neste regime, definimos $\hbar \omega$ de outra forma. Para ondas $\ell$ com $V_{B \ell}>E$ adotamos o método WKB, mais apropriado:

$$
T_{\ell}=\left[1+\exp \left(S_{\ell}\right)\right]^{-1}
$$




$$
S_{\ell}=\int_{R_{1}}^{R_{2}} \sqrt{\frac{8 \mu}{\hbar^{2}}\left[V_{\mathrm{ef}}(R)-E\right]} d R,
$$

onde $R_{1}$ e $R_{2}$ são os pontos de retorno clássicos. Neste caso, obtemos a curvatura da barreira que, dentro do contexto da expressão de Hill-Wheeler, forneceria o mesmo valor para o coeficiente de transmissão que aquele obtido com os cálculos de WKB para o potencial efetivo não-deformado:

$$
\hbar \omega_{\ell}=\frac{2 \pi\left(V_{B \ell}-E\right)}{S_{\ell}}
$$

Então, usamos este valor na Eq. (2.61) que contém o efeito dos acoplamentos. Com este método, assumimos que mesmo para $E \ll V_{B \ell}$ o potencial efetivo é bem descrito por uma barreira parabólica.

A Figura 3.6, na página 33, mostra uma representação esquemática da colisão de dois núcleos deformados, onde $\theta_{i}$ representa a direção do eixo de simetria do núcleo $i$ e $\vec{R}$ conecta o centro de massa dos dois núcleos. Devido ao curto alcance da interação nuclear, a altura da barreira depende praticamente apenas da coordenada $s$, representada na mesma figura. Diferentes conjuntos de valores de $\theta$ e $\beta$ que resultam no mesmo valor de $s$ também fornecem valores de $V_{B \ell}$ bastante similares. Logo, obtivemos amplitudes de acoplamento considerando a derivada da altura da barreira em relação à coordenada $s$ :

$$
F_{\lambda i}(\ell)=-\frac{\partial V_{B \ell}}{\partial s_{\lambda i}} .
$$

onde $i$ pode representar o núcleo 1 ou 2 e $\lambda$ representa o modo de vibração. No modelo vibracional, o desvio padrão da coordenada $s$ é conectado com o valor do parâmetro de deformação $\beta$ através de:

$$
\sigma_{\lambda i}=\frac{\beta_{\lambda i} R_{0 i}}{\sqrt{4 \pi}}
$$

Neste contexto, a colisão de dois núcleos deformados envolve quatro coordenadas vibracionais $\left(s_{\lambda i}\right)$ correspondendo ao modos de quadrupolo e octupolo de ambos os núcleos. Como uma aproximação, assumimos que a constantes de acoplamento $F_{\lambda i}$ são parâmetros independentes e a derivada parcial envolvida na Eq. (4.7) foi calculada em relação a uma forma esférica.

Do ponto de vista numérico, primeiramente calculamos os potenciais não-deformados, e suas correspondentes altura e curvatura de barreira, $V_{B}(\ell, E)$ e $\hbar \omega(\ell, E)$. Estes parâmetros são dependentes 
da energia devido à dependência em energia do potencial de SP e também aos cálculos de WKB da Eq. (4.5).

A seguir as intensidades de acoplamento $\left(F_{\lambda i}\right)$ são obtidas. Estes cálculos envolvem potenciais deformados e consomem bastante tempo de processamento. Logo, de maneira a economizar este tempo de processamento, os potenciais são calculados apenas para um valor de energia (o valor médio de energia do intervalo envolvido). Este procedimento é apropriado quando o intervalo de energia não é muito grande pois a dependência em energia do potencial de SP não é muito forte. Para um dado par de valores de $\lambda$ (modo de deformação) e $i$ (núcleo), as alturas de barreira do potencial deformado são obtidas considerando quatro configurações: $+2 \beta_{\lambda i},+\beta_{\lambda i},-\beta_{\lambda i} \mathrm{e}-2 \beta_{\lambda i}$, assumindo $\theta_{1}=\theta_{2}=0$ (ver Figura 3.6, página 33). Obtemos, então:

$$
\frac{\partial V_{B}}{\partial \beta_{\lambda i}} \approx \frac{-V_{B}\left(2 \beta_{\lambda i}\right)+8 V_{B}\left(\beta_{\lambda i}\right)-8 V_{B}\left(-\beta_{\lambda i}\right)+V_{B}\left(-2 \beta_{\lambda i}\right)}{12 \beta_{\lambda i}}
$$

As intensidades são, então, obtidas a partir de:

$$
F_{\lambda i}=-\frac{\partial V_{B}}{\partial s_{\lambda i}}=-\frac{\frac{\partial V_{B}}{\partial \beta_{\lambda i}}}{R_{0 i} Y_{\lambda 0}\left(\theta_{i}=0\right)}
$$

Enfatizamos que os fatores de forma e intensidades de acoplamento usados em nossos cálculos, descritos pelas Eqs. (4.9) e (4.10), foram obtidos como derivadas numéricas do potencial de São Paulo deformado, o que constitui maior acurácia do que o procedimento usualmente adotado de efetuar derivadas da forma esférica ou de alguma outra aproximação do potencial.

No caso dos cálculos numéricos de ZPM, temos que a soma quadridimensional da Eq. (2.59) (página 22) foi realizada considerando um certo intervalo para cada modo de vibração. A distribuição de Poisson dada pela Eq. (2.40) (página 18) possui ${ }^{1}$ valor médio $\langle n\rangle=\left(F \sigma / E^{*}\right)^{2}$ e desvio padrão $\sigma_{n}=\sqrt{n}$. Se $\langle n\rangle+3 \sigma_{n} \leq 50$, a soma da Eq. (2.59) é feita de $n=0$ até $n=50$, com passo $\Delta n=1$. Caso contrário, o intervalo $\langle n\rangle-3 \sigma_{n} \leq n \leq\langle n\rangle+3 \sigma_{n}$ é assumido, com passo $\Delta n=6 \sigma_{n} / 50$ (truncado para um valor inteiro). Neste caso, os fatores de peso são obtidos considerando que a

\footnotetext{
${ }^{1} \mathrm{~A}$ dedução dos fatores de peso representados pela distribuição de Poisson $W_{n}$ e de seu respectivo valor médio e desvio padrão pode ser encontrada de forma bastante detalhada no Apêndice B.
} 
distribuição de Poisson pode ser substituída pela Gaussiana correspondente:

$$
W_{n}=\frac{1}{\sqrt{2 \pi \sigma_{n}^{2}}} \mathrm{e}^{-\frac{(n-\langle n\rangle)^{2}}{2 \sigma_{n}^{2}}}
$$

Assumimos inicialmente $\ell_{\text {máx }}=300$, mas este valor é reduzido se um poço (e uma barreira) no potencial efetivo não é encontrado para algum valor de $\ell$.

\subsection{Comparação entre os Modelos}

Considerando a sistemática para comprimentos de deformação de quadrupolo e octupolo [38], baseada nos correspondentes resultados experimentais para as probabilidades de transição, e descrita na Seção 3.2, adotamos como parâmetros de deformação para o ${ }^{64} \mathrm{Ni}$ os seguintes valores: $\beta_{2}=0.215$, $\beta_{3}=0.263$. As freqüências dos modos $\lambda=2$ e 3 são conectadas com as energias de excitação dos correspondentes estados $2_{1}^{+}$e $3_{1}^{-}: E_{2}^{*} \approx 1.35 \mathrm{MeV}, E_{3}^{*} \approx 3.56 \mathrm{MeV}$ para o ${ }^{64} \mathrm{Ni}$. Dentro do contexto da sistemática para as densidades, o potencial de SP não possui nenhum parâmetro ajustável. Conseqüentemente, os resultados aqui apresentados para seções de choque representam previsões ao invés de ajustes de dados. Esta é uma importante característica de nosso modelo.

Nos modelos de ZPM e GFA, negligenciamos o fato de que a excitação de cada modo de superfície corresponde a uma transferência finita de momento angular. Os cálculos se tornariam muito mais complicados se tais efeitos fossem incluídos. Este procedimento já foi adotado em outros trabalhos (e.g. Ref. [62]) pois os efeitos de transferência de momento angular tem pouca influência na seção de choque total para colisões de sistemas pesados, como discutido na Ref. [63].

A Figura 4.1 apresenta o sistema ${ }^{64} \mathrm{Ni}+{ }^{64} \mathrm{Ni}$, analisado à luz de diferentes modelos. Três diferentes conjuntos de dados, que em alguns casos diferem entre si por um fator em torno de 3 , são mostrados com diferentes símbolos. A linha pontilhada na figura indica o resultado do BPM nãodeformado. Um enhancement de três ordens de magnitude dos dados em relação aos cálculos de BPM é claramente observado em energias abaixo da barreira. A linha traço-ponto duplo representa os resultados de FA, a linha traço-ponto simples representa os cálculos de GFA, e a linha cheia corresponde às previsões de ZPM. Em baixas energias, o efeito das energias de excitação reduz a seção de choque em várias ordens de grandeza em relação aos resultados de FA (ver Figura 4.1). Tanto os 
cálculos de GFA como os de ZPM estão em bom acordo com os dados.

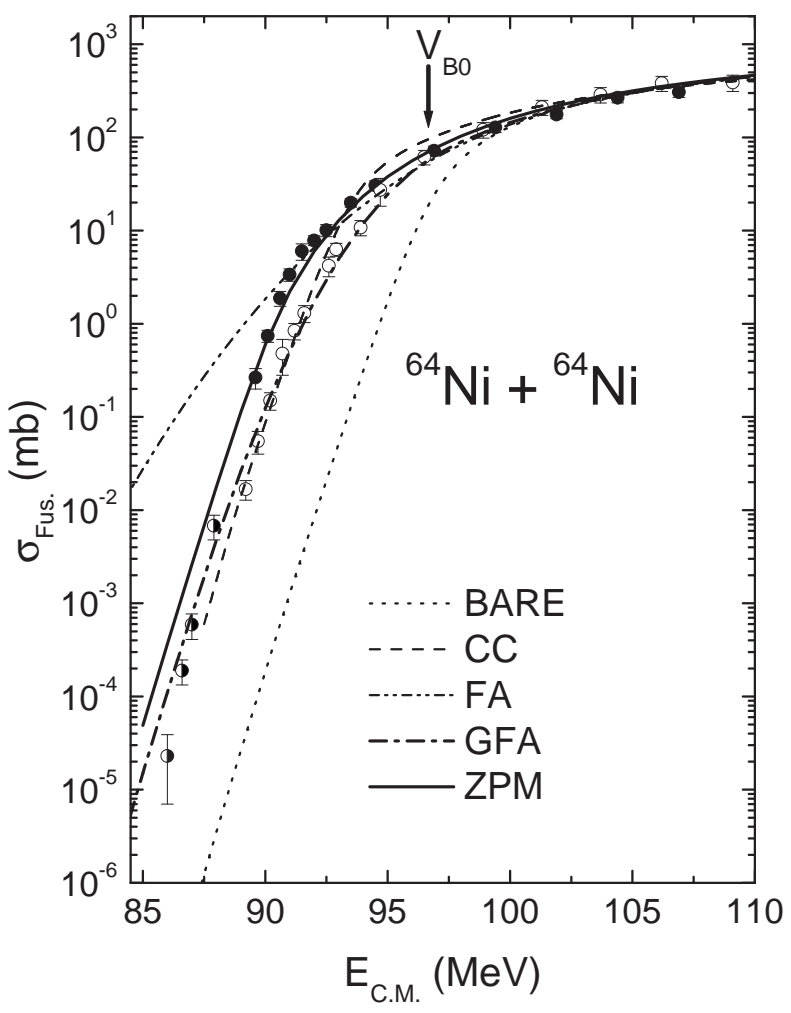

Dados obtidos das Refs. [8, 56, 57]

Figura 4.1: Dados experimentais e correspondentes funções de excitação de fusão para o sistema ${ }^{64} \mathrm{Ni}$ $+{ }^{64} \mathrm{Ni}$. As linhas correspondem a diferentes abordagens teóricas para o cálculo das seções de choque teóricas (ver texto para detalhes). A posição da altura da barreira para a onda $\mathrm{s}$ do potencial não deformado $\left(V_{B 0}\right)$ é mostrada na figura. Existem diferentes conjuntos de dados (representados na figura por diferentes símbolos) obtidos em diferentes trabalhos, com seções de choque que diferem entre si por um fator em torno de 3 para algumas energias.

Com o intuito de comparação, também realizamos cálculos usuais de CC considerando acoplamentos aos estados $2_{1}^{+}$e $3_{1}^{-}$e também aos estados do tripleto, associado à excitação quadrupolar de dois fônons, $0_{2}^{+}\left(E^{*}=2.94 \mathrm{MeV}\right), 2_{2}^{+}\left(E^{*}=2.78 \mathrm{MeV}\right), 4_{1}^{+}\left(E^{*}=2.46 \mathrm{MeV}\right)$, usando o código FRESCO [64]. Nestes cálculos usamos um potencial imaginário interno, para simular a absorção de fluxo pelo processo de fusão, com uma forma do tipo Woods-Saxon com $W_{0}=300 \mathrm{MeV}, r_{i 0}=0.8$ fm e $a_{i}=0.2 \mathrm{fm}$. O resultado correspondente é mostrado na Figura 4.1 por uma linha tracejada, e mostra uma concordância razoável com os cálculos de GFA e ZPM.

Na verdade, não é esperado que os cálculos de CC, GFA e ZPM forneçam exatamente os mesmos 
resultados, o que poderia levar à questão acerca de qual poderia ser considerado "melhor". Em uma primeira análise, poderia-se concluir que os cálculos "exatos" de CC são melhores, visto que os modelos de GFA e ZPM foram construídos para simular o efeito dos acoplamentos apenas de uma forma aproximada. Logo, obviamente os modelos que propomos representam somente uma solução aproximada das equações de CC. No entanto, os cálculos de CC são "exatos" apenas de um ponto de vista teórico. Na prática, como ocorre com qualquer formulação para o complicado problema da fusão de íons pesados, incluindo os modelos que apresentamos, diversas aproximações são assumidas nos cálculos de CC acerca de, por exemplo, fatores de forma, potenciais imaginários internos, etc. Um ponto importante é o fato de que os cálculos de CC são realizados considerando apenas um pequeno conjunto de estados, quase certamente os mais importantes. Os modelos de ZPM e de GFA, no entanto, levam em consideração a base completa de estados excitados conectados com os modos de oscilação e isto pode ser uma vantagem em certos casos onde um número grande de canais acoplados é necessário para o cálculo acurado dos coeficientes de transmissão. Poderíamos efetuar cálculos de CC envolvendo mais estados do que fizemos aqui. Porém, isto pode introduzir problemas numéricos na solução das equações acopladas. Para ilustrar este ponto, efetuamos cálculos de CC com o código FresCo [64] incluindo acoplamentos inelásticos apenas aos estados $2^{+}$e $3^{-}$do ${ }^{64} \mathrm{Ni}$. Os resultados destes cálculos, mostrados na Figura 4.2, não foram apresentados para $E_{C . M}$. $<$ 87.5 MeV, pois, para energias muito baixas, os cálculos numéricos não convergem nem apresentam seções de choque confiáveis. Tais problemas numéricos estão relacionados com o método normal radial progressivo para resolver as equações de CC que falha quando canais demais estão localmente fechados, resultando integração inacurada através da região classicamente proibida ( $E \ll V_{\text {ef }}$ ) para tais canais. No FrESCO, a fusão é calculada como sendo a diferença entre a seção de choque de reação e a soma das seções de choque de espalhamento inelástico. Isto significa que em baixas energias, quando as seções de choque de reação e de espalhamento são quase iguais, a fusão é uma pequena diferença de números grandes e, conseqüentemente, não pode ser calculada acuradamente. Para ilustrar este ponto, na Figura 4.3 mostramos a contribuição parcial de cada onda $\ell$ para a seção de choque total de fusão de $\mathrm{CC}$ em diferentes energias. Para $E=89 \mathrm{MeV}$, o comportamento da seção de choque parcial de fusão se torna oscilatório para $\ell \geq 18$, incluindo alguns valores negativos para particulares valores de $\ell$, o que é uma indicação de que ocorreram problemas numéricos. Mesmo 
assim, para esta energia um valor confiável para a seção de choque de fusão pode ser obtido se for considerada a soma sobre ondas parciais somente até $\ell=18$. Para a energia mais baixa da Figura 4.3, $E=87 \mathrm{MeV}$, as oscilações são muito maiores, com diversos valores negativos para a seção de choque parcial, e não há nenhuma forma de determinar uma seção de choque total de fusão confiável. Existem outras maneiras de resolver as equações de $\mathrm{CC}$, tais como usando expansões da matriz R, o que poderia eventualmente evitar este problema. Uma vantagem dos presentes modelos de GFA e ZPM é a ausência de problemas numéricos em tais condições. De fato, os cálculos de ZPM e GFA puderam ser realizados para energias muito baixas (ver Figura 4.2). Por outro lado, os modelos de ZPM e GFA assumem uma base infinita de estados excitados de vibrações harmônicas e, claramente, isto é apenas uma aproximação (e, conseqüentemente, uma limitação) para o complicado problema da colisão entre dois núcleos.

Para podermos avaliar o efeito do acoplamento à banda completa, também calculamos seções de choque considerando acoplamentos apenas aos estados $2^{+}$e $3^{-}$, mas em uma abordagem dentro do contexto do modelo de ZPM, assumindo as Eq. (2.43) e (2.44), como pode ser visto na Figura 4.2. Claramente estes resultados são bastante similares àqueles obtidos com o código FRESCO. Na mesma Figura 4.2, pode-se observar a diferença entre os procedimentos de acoplar apenas os estados $2^{+} \mathrm{e}$ $3^{-}$em comparação com os acoplamentos às bandas completas que correspondem a estes estados. A diferença seria muito mais significativa se as energias de excitação fossem menores, pois, neste caso, muitos estados contribuiriam significativamente para o processo de fusão.

Assim sendo, vemos que os modelos de GFA e ZPM propostos são bastante apropriados para o cálculos de seções de choque de fusão em regiões de energia onde o método de CC falha. Além disso, os modelos GFA e ZPM incluem, em suas previsões, acoplamentos a todos os estados das bandas vibracionais de quadrupolo e octupolo. 


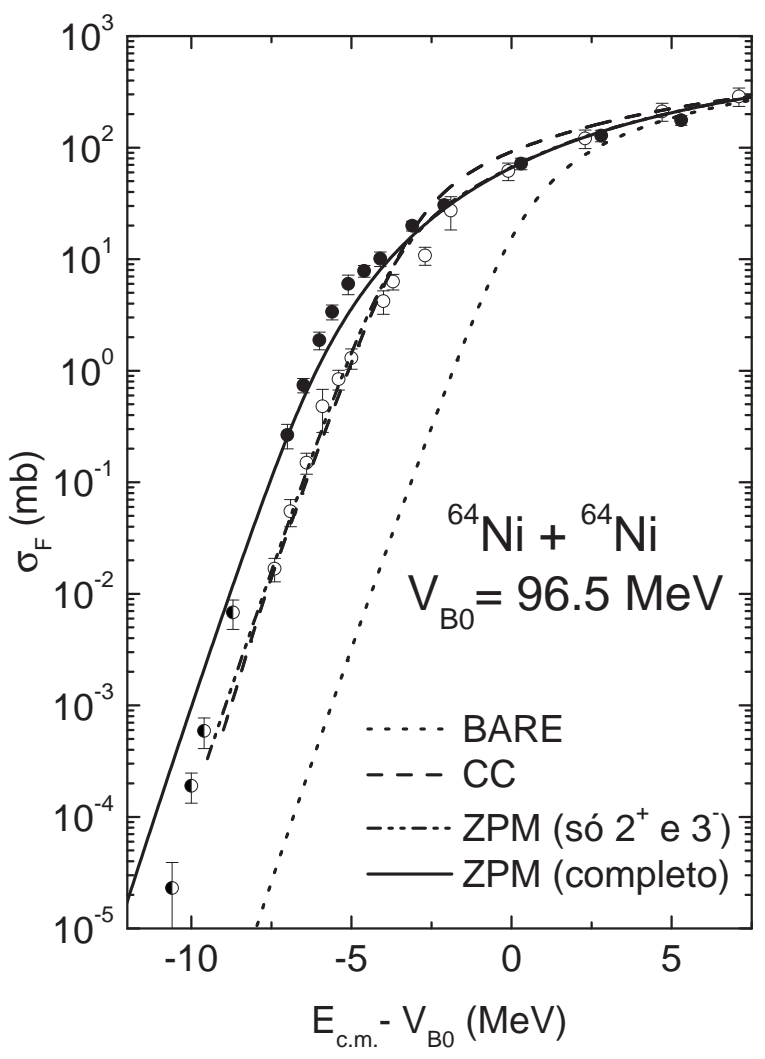

Dados obtidos das Refs. [8, 56, 57]

Figura 4.2: Dados experimentais e correspondentes seções de choque teóricas de BPM não-deformada (linha tracejada) e de ZPM considerando as bandas completas (linha cheia) para o sistema ${ }^{64} \mathrm{Ni}+$ ${ }^{64} \mathrm{Ni}$. As outras linhas indicam cálculo de CC com o código FRESCO e com o modelo representado pelas Eqs. (2.43) e (2.44), onde apenas os acoplamentos aos estados $2^{+}$e $3^{-}$foram considerados. Existem diferentes conjuntos de dados (representados na figura por diferentes símbolos) obtidos em diferentes trabalhos, com seções de choque que diferem entre si por um fator em torno de 3 para algumas energias. 

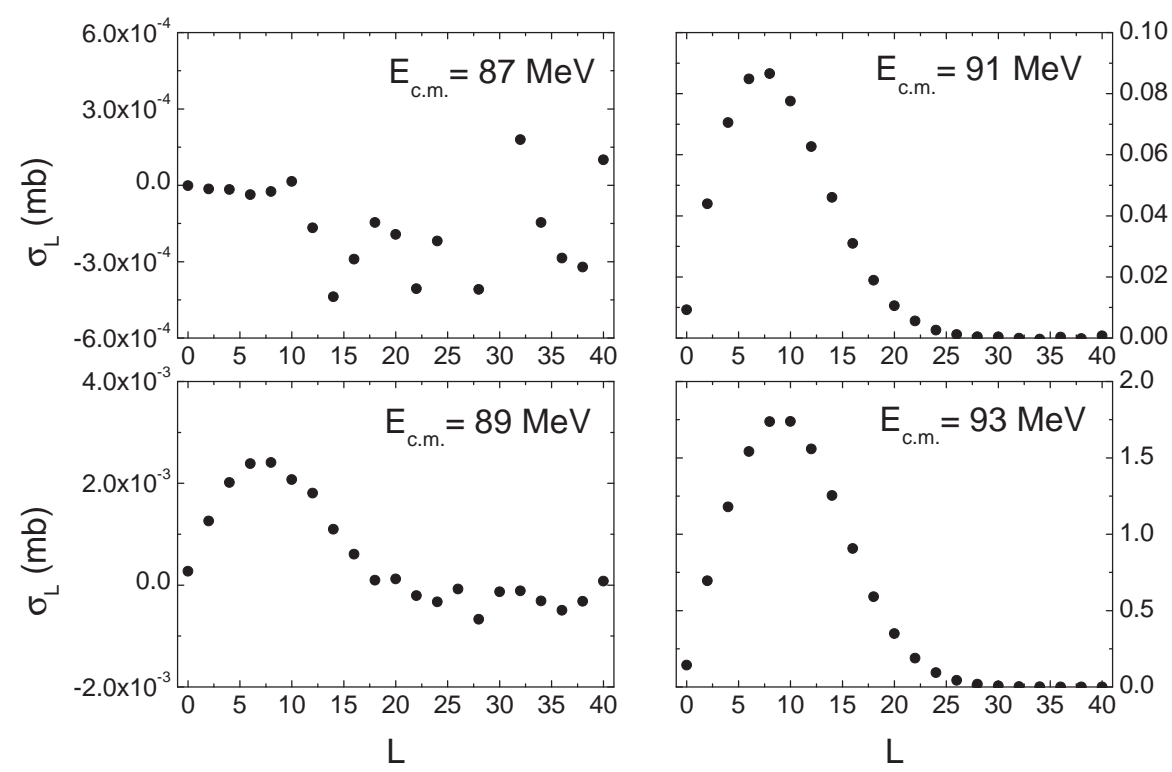

Figura 4.3: Seções de choque parcial de fusão em quatro energias, obtidas de cálculos de CC com o código FRESCO para o sistema ${ }^{64} \mathrm{Ni}+{ }^{64} \mathrm{Ni}$. 


\section{Capítulo 5}

\section{Sistemática de Fusão para Núcleos Par-Par}

Como vimos no Capítulo 4, os modelos de Movimento de Ponto Zero (ZPM) e Aproximação Congelada Generalizada (GFA) são equivalentes no cálculo de seções de choque de fusão. Desta forma, neste capítulo, aplicamos apenas o modelo de ZPM, cuja versão unidimensional é descrita em detalhes na Seção 2.4, para o cálculo da seção de choque de fusão para 64 diferentes sistemas, desde os mais leves, como ${ }^{12} \mathrm{C}+{ }^{12} \mathrm{C}$, até sistemas bem pesados. Esta sistemática, apresentada neste capítulo e que pode ser encontrada na Ref. [36], foi realizada sempre comparando as previsões do modelo proposto com os dados experimentais existentes na literatura.

O modelo de ZPM é aplicado no contexto descrito no Capítulo 4, ou seja, sem assumir nenhum parâmetro livre, que possa ser ajustado. Esta é uma característica importante pois, ao fazer isso para muitos sistemas, podemos identificar propriedades globais do processo de fusão para núcleos pesados.

\subsection{Definição dos Parâmetros do Modelo}

As constantes de acoplamento (fatores de forma), as curvaturas de barreira, os desvios padrão de vibração e a probabilidade de penetração da barreira em função das transmissões para cada onda parcial $\ell$ utilizados na presente sistemática foram calculados da mesma forma descrita na Seção 4.1, podendo ser sintetizados pelas Eqs. (4.2) a (4.8). O potencial efetivo, a partir do qual é definida a barreira parabólica, também é dado pela Eq. (4.1).

Os parâmetros de deformação coulombianos e nucleares podem não ter o mesmo valor, visto que 
são relacionados com diferentes distribuições (de carga e de matéria, respectivamente). No entanto, para evitar o uso de parâmetros ajustáveis, assumimos o mesmo valor para ambas deformações. Este procedimento é na verdade bastante razoável pois é esperado que as deformações coulombianas e nucleares tenham valores similares. Os valores de $\beta_{2}$ e $\beta_{3}$ foram obtidos pela Eq. (3.7), através de sistemática de amplitudes de transição para núcleos par-par, apresentada nas Refs. [51, 65].

No contexto aqui apresentado, o modelo de ZPM descreve, de uma forma aproximada, os acoplamentos de estados excitados relacionados a bandas completas de modos de vibração quadrupolar e octupolar, correspondendo a um grande número de acoplamentos a estados inelásticos. No entanto, nenhum acoplamento a canais de transferência foi incluído em nossos cálculos. Alguns dos núcleos envolvidos na presente sistemática poderiam ser melhor representados pelo modelo rotacional ao invés do vibracional. No entanto, o desvio padrão $\sigma_{\lambda}$ de distribuições rotacionais é relacionado com o valor de $\beta_{\lambda}$ correspondente através da mesma expressão que é válida para distribuições vibracionais, ou seja, Eq. (4.8). No entanto, as formas das distribuições para a coordenada $s$ nos modos rotacional e vibracional são diferentes. Mesmo assim, assumimos o modelo de ZPM para todos os sistemas com o objetivo de investigar os limites deste modelo na reprodução de dados de fusão.

\subsection{Comparação com os Dados Experimentais}

Na Tabela 5.1, apresentamos os núcleos envolvidos nos sistemas estudados na presente sistemática, com os correspondentes valores de $\beta$ e $E^{*}$ para os modos de quadrupolo e octupolo. As Figuras 5.1 a 5.7 e 5.9 apresentam os dados de fusão (provenientes das referências fornecidas nas legendas) e os correspondentes resultados dos cálculos através do modelo não-deformado de penetração de barreira (BPM), em linhas tracejadas, e do modelo de ZPM, em linhas cheias. A escala de energia é representada em relação à altura da barreira não-deformada da onda $s, V_{B 0}$. Com exceção das Figuras $5.1 \mathrm{e}$ 5.4, as figuras têm a mesma escala de energia e seção de choque. Este procedimento torna simples a comparação entre os resultados de diferentes sistemas.

Na Figura 5.1 apresentamos os sistemas mais leves estudados no presente capítulo. No caso do

${ }^{12} \mathrm{C}+{ }^{12} \mathrm{C}$, os resultados do BPM e do modelo de ZPM são quase indistinguíveis e concordam muito bem com os dados em toda a região de energia, correspondendo a aproximadamente nove ordens de 
Tabela 5.1: A tabela apresenta os valores dos parâmetros de deformação de quadrupolo $\left(\beta_{2}\right)$ e de octupolo $\left(\beta_{3}\right)$ e também as correspondentes energias de excitação para os núcleos estudados nesta sistemática.

\begin{tabular}{|c|c|c|c|c|}
\hline núcleo & $\beta_{2}$ & $\bar{E}_{2}^{*}(\mathrm{MeV})$ & $\beta_{3}$ & $E_{3}^{*}(\mathrm{MeV})$ \\
\hline${ }^{12} \mathrm{C}$ & 0.89 & 4.44 & 1.55 & 9.64 \\
\hline${ }^{16} \mathrm{O}$ & 0.50 & 6.92 & 1.20 & 6.13 \\
\hline${ }^{18} \mathrm{O}$ & 0.54 & 1.98 & 1.10 & 5.10 \\
\hline${ }^{28} \mathrm{Si}$ & 0.50 & 1.78 & 0.55 & 6.88 \\
\hline${ }^{30} \mathrm{Si}$ & 0.41 & 2.24 & 0.41 & 5.49 \\
\hline${ }^{32} \mathrm{~S}$ & 0.38 & 2.23 & 0.70 & 5.01 \\
\hline${ }^{36} \mathrm{~S}$ & 0.32 & 3.29 & 0.56 & 4.19 \\
\hline${ }^{40} \mathrm{Ar}$ & 0.32 & 1.46 & 0.58 & 3.68 \\
\hline${ }^{40} \mathrm{Ca}$ & 0.14 & 3.90 & 0.51 & 3.74 \\
\hline${ }^{48} \mathrm{Ca}$ & 0.13 & 3.83 & 0.34 & 4.51 \\
\hline${ }^{48} \mathrm{Ti}$ & 0.32 & 0.98 & 0.26 & 3.36 \\
\hline${ }^{58} \mathrm{Ni}$ & 0.21 & 1.45 & 0.24 & 4.48 \\
\hline${ }^{64} \mathrm{Ni}$ & 0.22 & 1.35 & 0.26 & 3.56 \\
\hline${ }^{68} \mathrm{Zn}$ & 0.24 & 1.08 & 0.30 & 2.75 \\
\hline${ }^{70} \mathrm{Ge}$ & 0.26 & 1.04 & 0.33 & 2.56 \\
\hline${ }^{76} \mathrm{Ge}$ & 0.32 & 0.56 & 0.19 & 2.69 \\
\hline${ }^{86} \mathrm{Kr}$ & 0.17 & 1.56 & 0.19 & 3.10 \\
\hline${ }^{90} \mathrm{Zr}$ & 0.10 & 2.19 & 0.25 & 2.75 \\
\hline${ }^{92} \mathrm{Zr}$ & 0.12 & 0.93 & 0.21 & 2.34 \\
\hline${ }^{96} \mathrm{Zr}$ & 0.10 & 1.75 & 0.36 & 1.90 \\
\hline${ }^{92} \mathrm{Mo}$ & 0.12 & 1.51 & 0.19 & 2.85 \\
\hline${ }^{94} \mathrm{Mo}$ & 0.17 & 0.87 & 0.18 & 2.53 \\
\hline${ }^{98} \mathrm{Mo}$ & 0.19 & 0.79 & 0.27 & 2.02 \\
\hline${ }^{100} \mathrm{Mo}$ & 0.27 & 0.54 & 0.27 & 1.91 \\
\hline${ }^{102} \mathrm{Ru}$ & 0.28 & 0.48 & 0.17 & 2.04 \\
\hline${ }^{104} \mathrm{Ru}$ & 0.32 & 0.36 & 0.16 & 1.97 \\
\hline${ }^{108} \mathrm{Pd}$ & 0.28 & 0.43 & 0.20 & 2.05 \\
\hline${ }^{110} \mathrm{Pd}$ & 0.30 & 0.37 & 0.19 & 2.04 \\
\hline${ }^{112} \mathrm{Sn}$ & 0.14 & 1.26 & 0.15 & 2.36 \\
\hline${ }^{116} \mathrm{Sn}$ & 0.13 & 1.29 & 0.18 & 2.27 \\
\hline${ }^{122} \mathrm{Sn}$ & 0.12 & 1.14 & 0.15 & 2.49 \\
\hline${ }^{124} \mathrm{Sn}$ & 0.11 & 1.13 & 0.13 & 2.60 \\
\hline${ }^{144} \mathrm{Sm}$ & 0.10 & 1.66 & 0.17 & 1.81 \\
\hline${ }^{148} \mathrm{Sm}$ & 0.16 & 0.55 & 0.19 & 1.16 \\
\hline${ }^{152} \mathrm{Sm}$ & 0.35 & 0.12 & 0.12 & 1.04 \\
\hline${ }^{154} \mathrm{Sm}$ & 0.39 & 0.08 & 0.10 & 1.01 \\
\hline${ }^{182} \mathrm{~W}$ & 0.28 & 0.10 & 0.06 & 1.37 \\
\hline${ }^{186} \mathrm{~W}$ & 0.25 & 0.12 & 0.07 & 1.04 \\
\hline${ }^{192} \mathrm{Os}$ & 0.19 & 0.21 & 0.07 & 1.34 \\
\hline${ }^{194} \mathrm{Pt}$ & 0.16 & 0.33 & 0.07 & 1.43 \\
\hline${ }^{208} \mathrm{~Pb}$ & 0.06 & 4.08 & 0.13 & 2.61 \\
\hline${ }^{238} \mathrm{U}$ & 0.32 & 0.05 & 0.10 & 0.73 \\
\hline
\end{tabular}


magnitude em seção de choque. Para o sistema ${ }^{16} \mathrm{O}+{ }^{16} \mathrm{O}$, as seções de choque de ZPM são ligeiramente maiores do que as previsões do BPM e se aproximam apropriadamente dos dados em energias abaixo da barreira. Apesar dos altos valores de $\beta$ dos núcleos de ${ }^{12} \mathrm{C}$ e ${ }^{16} \mathrm{O}$ (ver Tabela 5.1), as previsões do ZPM e do BPM são bastante similares e quase nenhum efeito dos acoplamentos é observado. Isto ocorre porque a intensidade de acoplamento $F$ para sistemas leves é pequena e as energias de excitação para estes núcleos são grandes. Para ilustrar este ponto, as linhas pontilhadas na Figura 5.1 representam os resultados da aproximação congelada (FA, do inglês Frozen Approximation), i.e. aqueles obtidos considerando $E^{*}=0$. Uma descrição mais detalhada sobre o modelo de FA, incluindo as expressões para o cálculo da transmissão, pode ser encontrada na Seção 2.2. Comparando as linhas cheias e pontilhadas, pode-se notar o efeito significativo da energia de excitação.

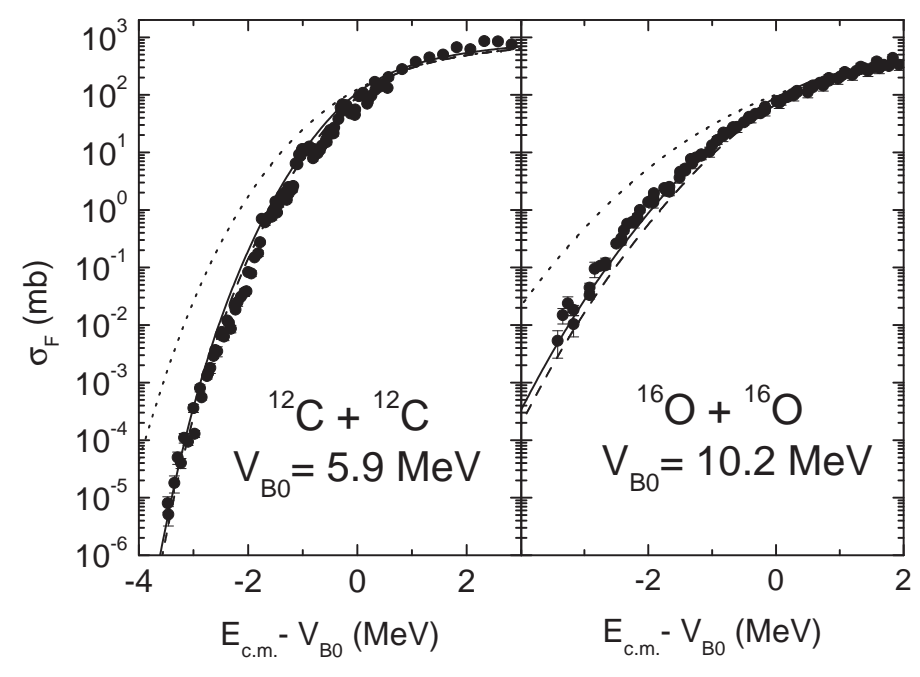

Dados obtidos das Refs. [66-74].

Figura 5.1: Dados experimentais de fusão e correspondentes seções de choque teóricas para BPM nãodeformado (linhas tracejadas) e para o modelo de ZPM (linhas cheias) para os sistemas ${ }^{12} \mathrm{C}+{ }^{12} \mathrm{C} e$ ${ }^{16} \mathrm{O}+{ }^{16} \mathrm{O}$. As linhas pontilhadas representam os resultados de FA, i.e. aqueles obtidos considerando energias de excitação nulas. As alturas das barreiras dos potenciais não-deformados para as ondas $\mathrm{s}$ são apresentadas na figura.

A Figura 5.2(a) apresenta dados de fusão para os sistemas ${ }^{12} \mathrm{C}+{ }^{92} \mathrm{Zr}$ e ${ }^{12} \mathrm{C},{ }^{18} \mathrm{O},{ }^{32} \mathrm{~S}+{ }^{208} \mathrm{~Pb}$. Grandes enhancements dos dados em relação às seções de choque de BPM podem ser observados, e os resultados de ZPM estão em muito melhor concordância com os dados. Os sistemas mais pesados apresentam enhancements maiores do que os sistemas mais leves, como já foi relatado muitos anos 
atrás [2]. Este comportamento ocorre devido ao aumento dos valores das intensidades de acoplamento $(F)$ para sistemas mais pesados. No entanto, este, na verdade, não é um comportamento geral da fusão de íons pesados e algumas exceções podem ser encontradas. Por exemplo, na Figura 5.2(b) vemos que o enhancement para o sistema ${ }^{16} \mathrm{O}+{ }^{208} \mathrm{~Pb}$ é, na verdade, ligeiramente menor que aqueles para os sistemas mais leves ${ }^{16} \mathrm{O}+{ }^{182,186} \mathrm{~W}$. Por outro lado, as seções de choque de ZPM apresentam boa concordância com os dados (ver Figura 5.2(b)). Este comportamento está relacionado com os valores maiores de $\beta_{2}$ (e menores $E_{2}^{*}$ ) do ${ }^{182,186} \mathrm{~W}$ em comparação com o ${ }^{208} \mathrm{~Pb}$ (ver Tabela 5.1).

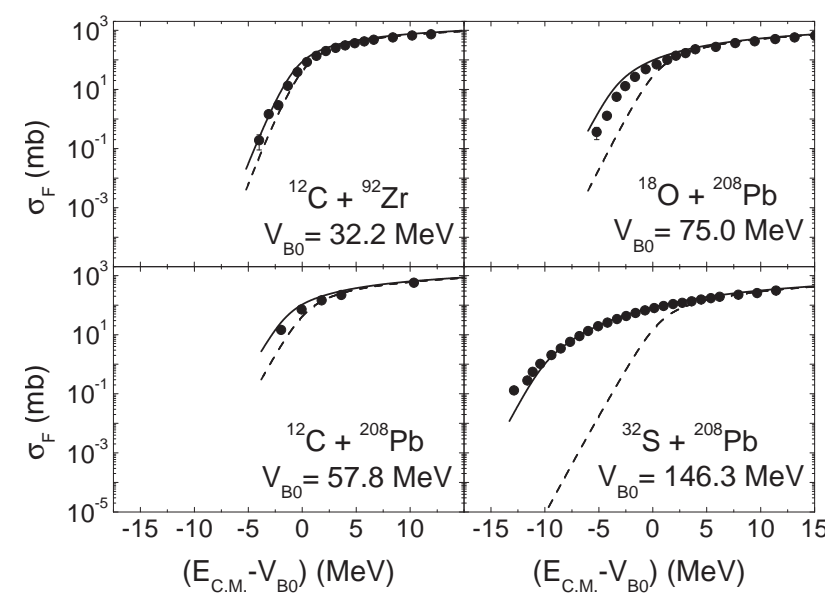

(a) Os dados são das Refs. [75-78].

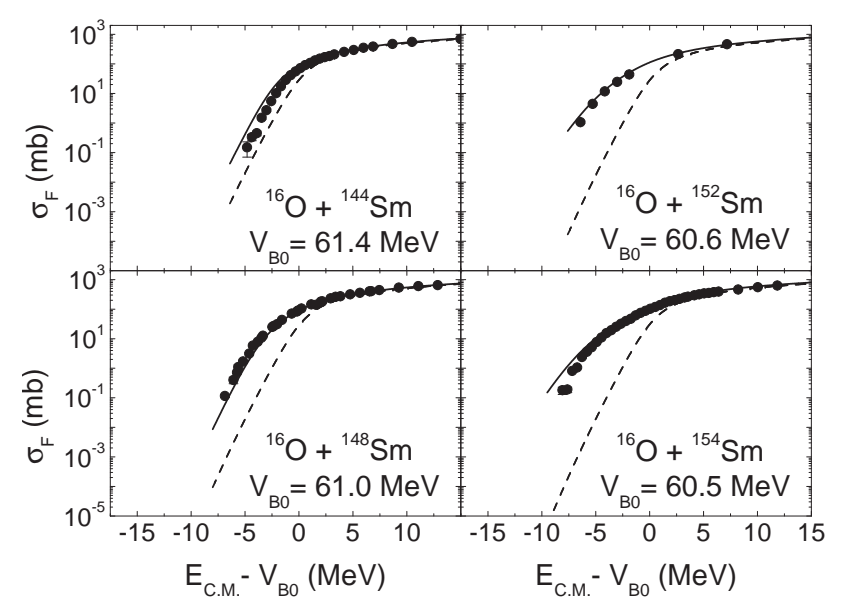

(c) Os dados são das Refs. [79, 83].

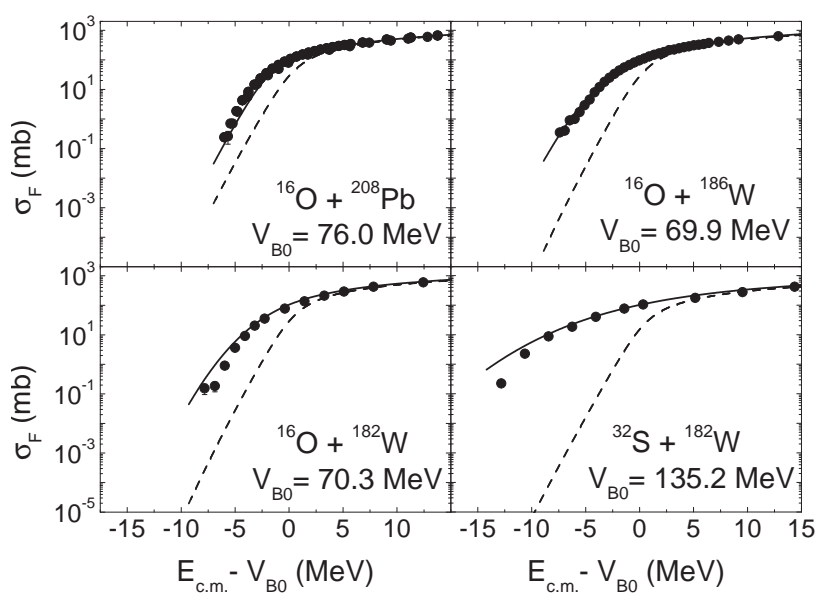

(b) Os dados são das Refs. [77, 79-82].

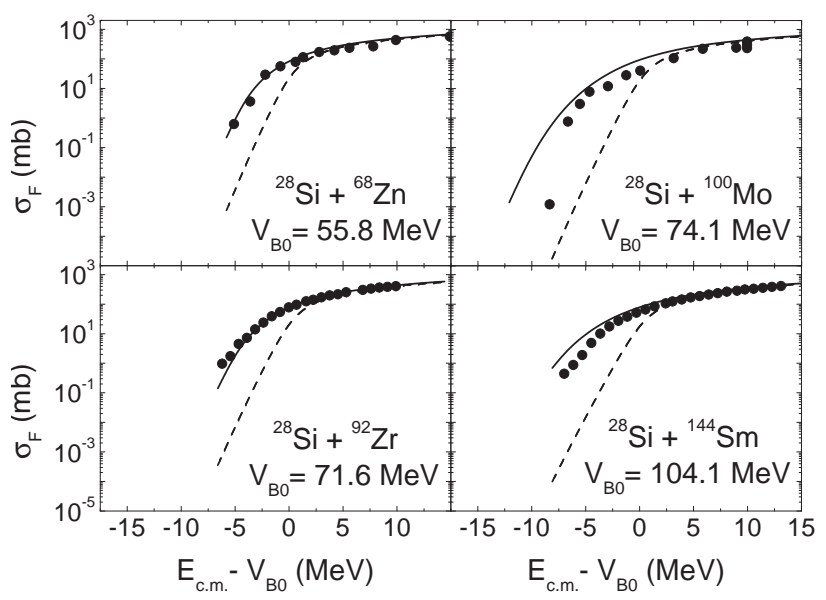

(d) Os dados são das Refs. [56, 75, 84, 85].

Figura 5.2: Dados de fusão e correspondentes seções de choque teóricas para BPM não-deformado (linhas tracejadas) e para o modelo de ZPM (linhas cheias) para os sistemas indicados nas figuras. As alturas das barreiras dos potenciais não-deformados para as ondas s são apresentadas na figura. 
A Figura 5.2(c) mostra sistemas com o projétil ${ }^{16} \mathrm{O}$ em alvos que correspondem a diferentes isótopos de samário. Apesar da região de massa ser similar, diferenças significativas na magnitude dos enhancements (em relação ao BPM) são observadas, indicando fortes efeitos de estrutura dos núcleos alvos. Este sistemas foram analisados na Ref. [12] onde, pela primeira vez, o movimento de ponto zero da superfície nuclear foi aplicado no estudo da fusão de íons pesados. Naquele trabalho, o efeito da energia de excitação não foi considerado e um parâmetro ajustável relacionado ao potencial bare foi usado para ajustar os dados. Claramente, os presentes resultados de ZPM também levam em conta estes efeitos de estrutura devido aos valores bem diferentes de $\beta_{2}$ e $E_{2}^{*}$ dos isótopos de samário (ver Tabela 5.1).

A Figura 5.2(d) apresenta sistemas que envolvem ${ }^{28} \mathrm{Si}$ como projétil. As Figuras 5.3(a), 5.3(b) e 5.3(c) mostram sistemas com ${ }^{32,36} \mathrm{~S}$. As Figuras 5.3(d), 5.3(e) e 5.3(f) correspondem a sistemas envolvendo ${ }^{40,48} \mathrm{Ca} \mathrm{e}{ }^{40} \mathrm{Ar}$. A concordância geral entre dados e resultados de ZPM é boa e comparável com os resultados apresentados nas anteriores Figuras 5.1 e 5.2.

Na Figura 5.4 apresentamos o sistema ${ }^{40} \mathrm{Ar}+{ }^{144} \mathrm{Sm}$. As contribuições dos processos de resíduos de evaporação (círculos abertos) e de fusão-fissão (círculos semi-abertos) para a seção de choque total de fusão (círculos fechados) também são apresentadas. Para sistemas muito pesados o processo de fusão-fissão é responsável por uma parte importante da seção de choque total de fusão. Como esperado, os resultados de ZPM representam a fusão total (ver Figura 5.4) visto que estes resultados estão associados à absorção completa de fluxo advinda do tunelamento de barreira. Como outro exemplo, na Figura 5.5(a) alguns sistemas envolvendo o núcleo ${ }^{84} \mathrm{Kr}$ são apresentados. Para estes sistemas apenas as seções de choque de resíduo de evaporação foram medidas. A comparação entre dados e previsões teóricas indica que a contribuição de fusão-fissão se torna mais importante para sistemas mais pesados. De fato, enquanto para os alvos de germânio esta contribuição não aparenta ser significativa em toda região de energia, para o ${ }^{92}$ Mo claramente a fusão-fissão deve ser importante na região acima da barreira. Na verdade, para todos os sistemas apresentados anteriormente (Figuras 5.1 a 5.4), as previsões do modelo de ZPM e também as do BPM concordam bastante bem com os dados para energias acima da barreira, mas para o sistema ${ }^{84} \mathrm{Kr}+{ }^{92}$ Mo este comportamento não é observado. Para o sistema ${ }^{84} \mathrm{Kr}+{ }^{104} \mathrm{Ru}$ o processo de fusão-fissão pode ser importante também em energias abaixo da barreira (ver Figura 5.5(a)). Para sistemas simétricos, este efeito é ainda mais 


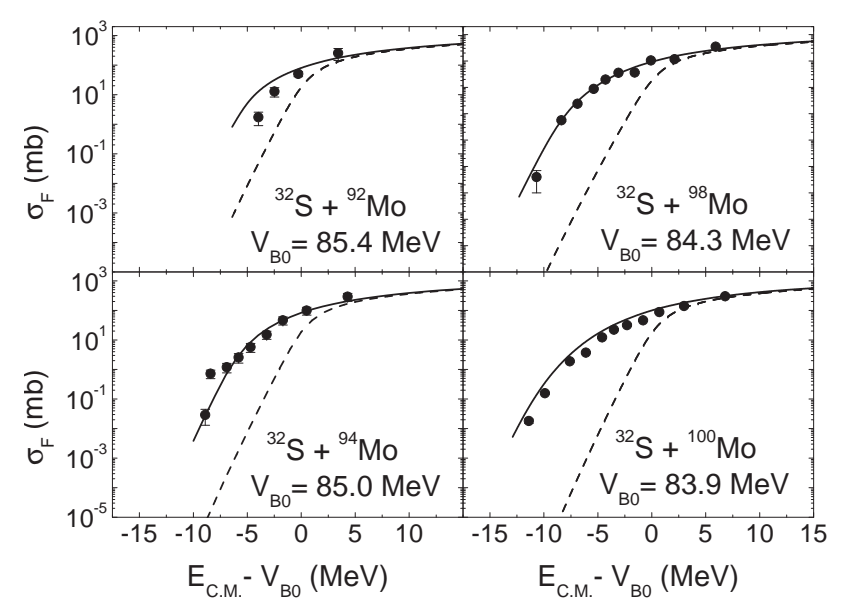

(a) Os dados são da Ref. [86].

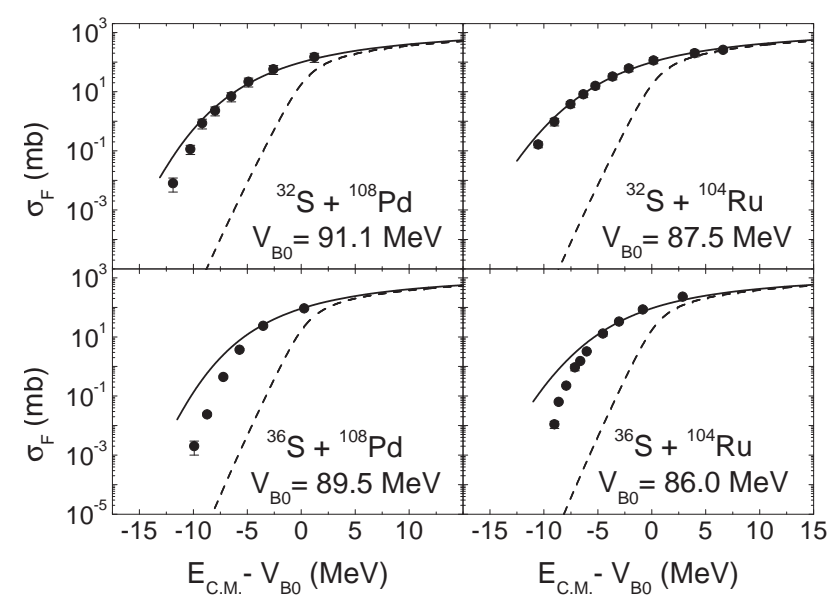

(c) Os dados são da Ref. [86].

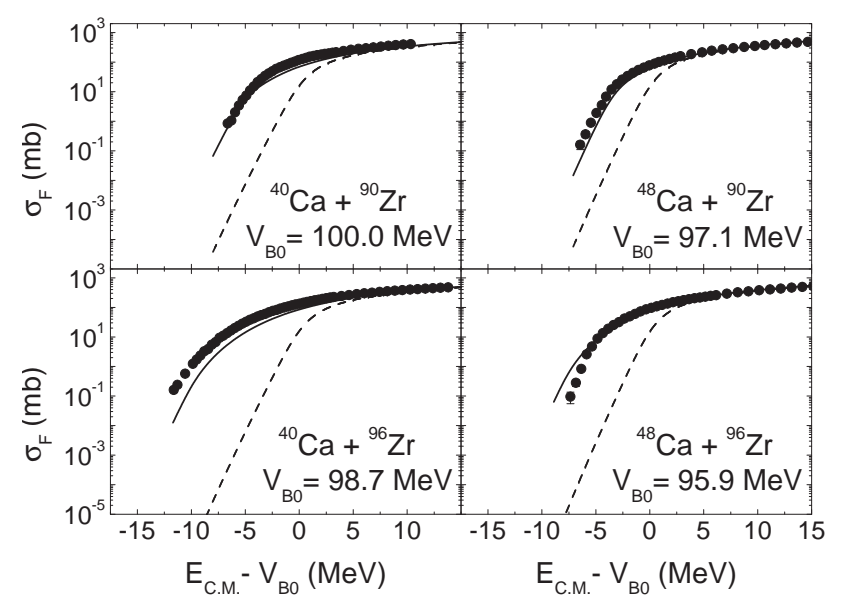

(e) Os dados são das Refs. [6,91].

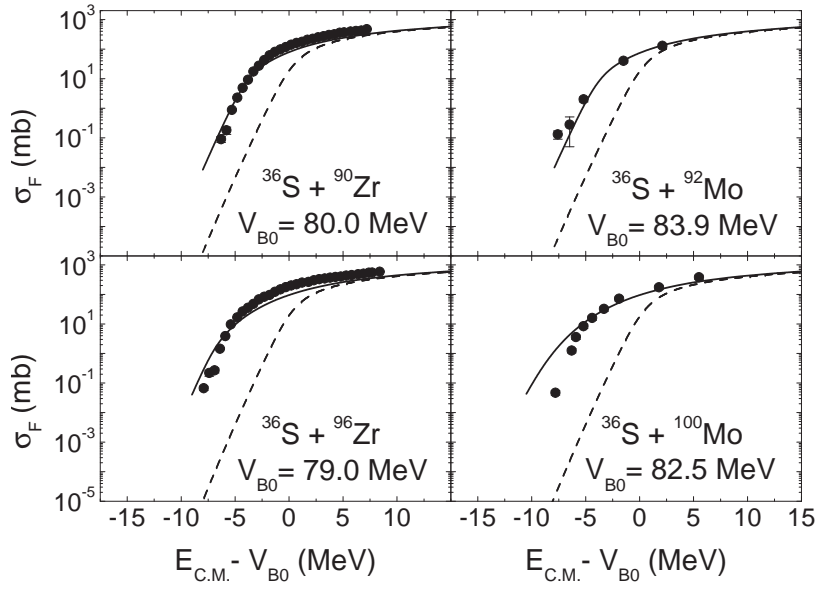

(b) Os dados são das Refs. [86, 87].

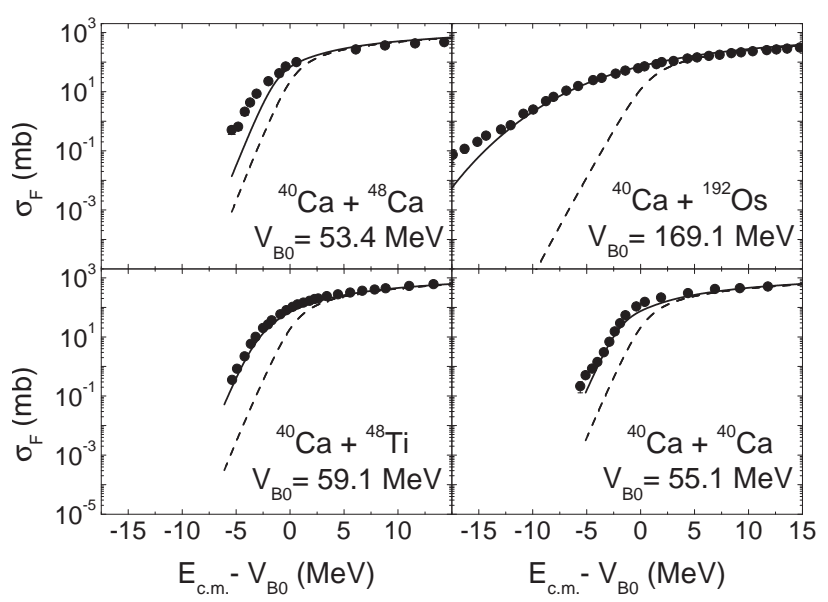

(d) Os dados são das Refs. [88-90].

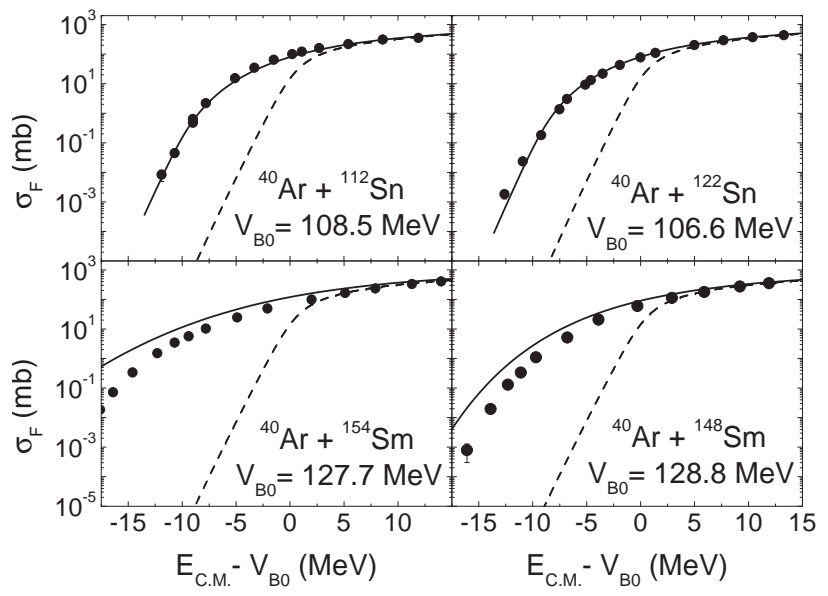

(f) Os dados são da Ref. [92].

Figura 5.3: Dados de fusão e correspondentes seções de choque teóricas para BPM não-deformado (linhas tracejadas) e para o modelo de ZPM (linhas cheias) para os sistemas indicados nas figuras. As alturas das barreiras dos potenciais não-deformados para as ondas s são apresentadas na figura. 


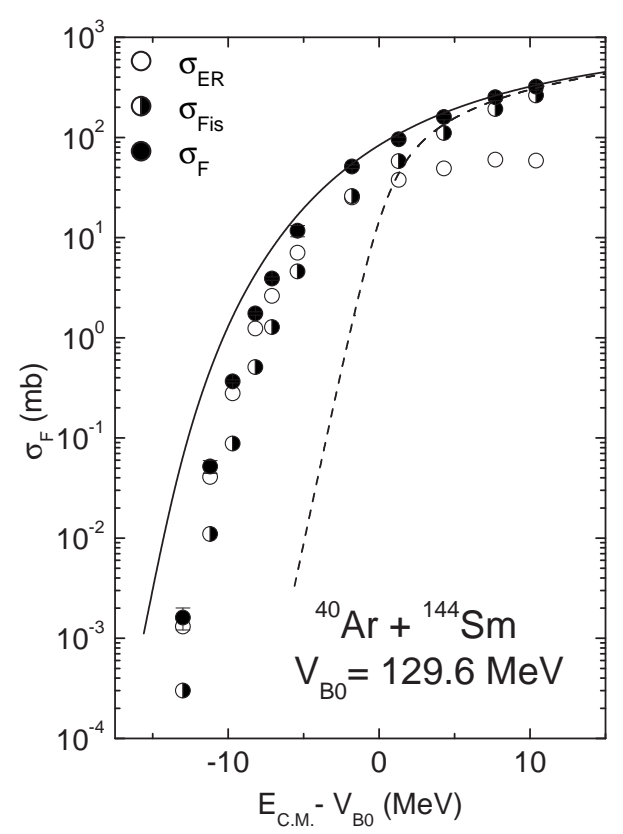

Dados experimentais obtidos da Ref. [92].

Figura 5.4: Seção de choque total de fusão (círculos fechados) para o sistema ${ }^{40} \mathrm{Ar}+{ }^{144} \mathrm{Sm}$. As contribuiçães de resíduo de evaporação (círculos abertos) e de fusão-fissão (círculos semi-abertos) para a fusão total também são apresentadas. As linhas tracejadas e cheias correspondem às seções de choque de BPM e ZPM, respectivamente.

enfatizado, como pode ser observado na Figura 5.5(b) para os sistemas ${ }^{90} \mathrm{Zr}+{ }^{90,96} \mathrm{Zr}$. Ressaltamos aqui, mais uma vez, a importância de não termos utilizado parâmetros livres em nossas análises. Uma inspeção da Figura 5.5(b) mostra claramente que os dados medidos para os sistemas ${ }^{90} \mathrm{Zr}+{ }^{90,96} \mathrm{Zr}$ não podem ser associados à seção de choque total de fusão, como já foi assumido em alguns trabalhos na literatura, e que a contribuição do processo de fusão-fissão deve ser bastante relevante.

Na Figura 5.6 outros sistemas são apresentados. As correspondentes seções de choque de ZPM representam bem o comportamento dos dados, exceto para os sistemas ${ }^{64} \mathrm{Ni}+{ }^{100} \mathrm{Mo} \mathrm{e}{ }^{58} \mathrm{Ni}+{ }^{112} \mathrm{Sn}$ (Figura 5.6(b)). Neste último caso, o dado de mais baixa energia tem seção de choque em torno de três ordens de grandeza maior que a previsão teórica correspondente. Por outro lado, para o sistema similar ${ }^{58} \mathrm{Ni}+{ }^{124} \mathrm{Sn}$ (Figura 5.6(b)) as seções de choque de ZPM estão em bom acordo com os dados. Discutimos as possíveis fontes destas significativas diferenças entre sistemas similares na Seção 5.3.

A Figura 5.7(a) apresenta dados de fusão e correspondentes resultados de BPM e ZPM para os sistemas ${ }^{16} \mathrm{O}+{ }^{238} \mathrm{U}$ e ${ }^{28} \mathrm{Si}+{ }^{208} \mathrm{~Pb}$. As seções de choque de ZPM estão em bom acordo com os dados. 


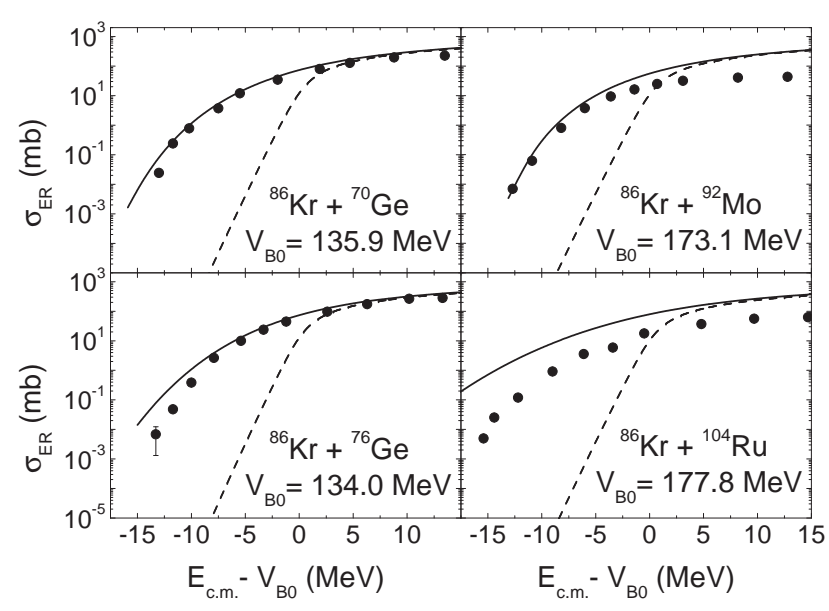

(a) Sistemas envolvendo ${ }^{86} \mathrm{Kr}$ como projétil. Os dados são da Ref. [93].

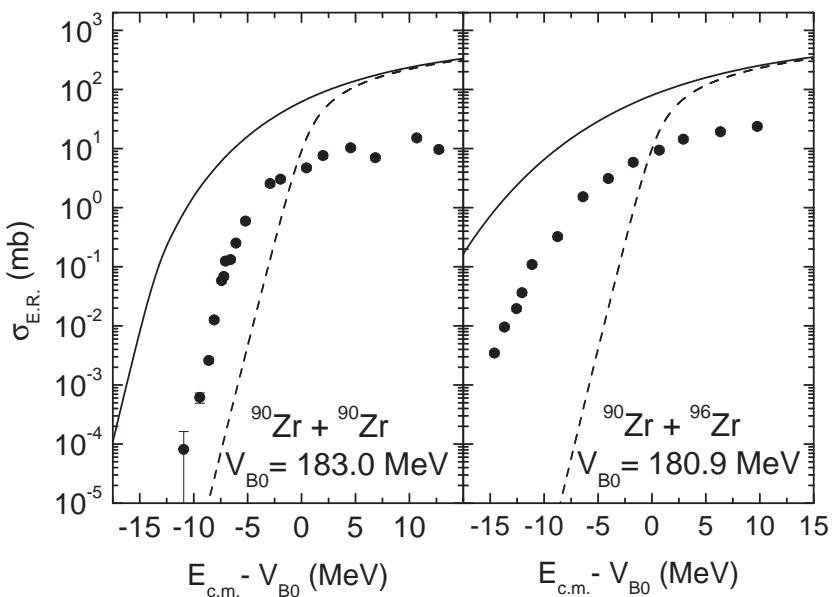

(b) Sistemas envolvendo ${ }^{90} \mathrm{Zr}$ como projétil. Os dados são da Ref. [94].

Figura 5.5: Seções de choque de resíduo de evaporação para diversos sistemas envolvendo ${ }^{86} \mathrm{Kr}$ e ${ }^{90} \mathrm{Zr}$ como projétil. As linhas tracejadas e cheias correspondem às seções de choque de BPM e ZPM, respectivamente.

As distribuições de barreira ${ }^{1}$ (DB) experimentais e teóricas são também apresentadas na parte de cima da figura. Claramente, o efeito dos acoplamentos torna as distribuições de ZPM (linhas cheias) mais larga do que aquelas que correspondem ao BPM (linhas tracejadas), resultando em uma concordância bastante razoável entre as DB experimentais e teóricas. Dois outros exemplos de DB são mostrados na Figura 5.7(b). Nestes casos, a DB apresenta estruturas complicadas enquanto a DB teórica fornece curvas suaves que correspondem ao comportamento médio da DB experimental.

\footnotetext{
${ }^{1}$ A distribuição de barreira de uma dada função de excitação para a fusão é definida como sendo $\frac{\mathrm{d}^{2} E \sigma}{\mathrm{d} E^{2}}$, onde $\sigma$ é a seção de choque de fusão. Ela fornece informações acerca do processo de fusão na região de energia em torno da barreira.
} 


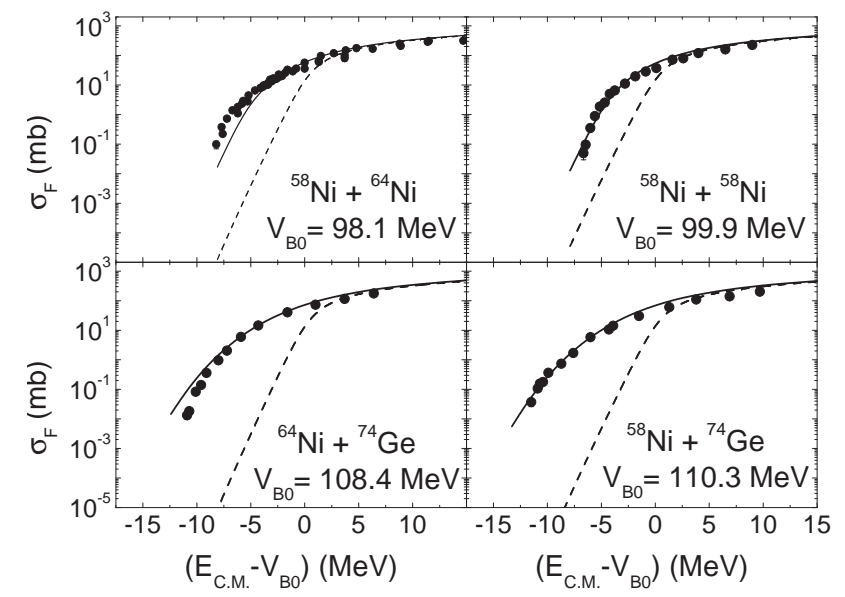

(a) Os dados são das Refs. [56, 57, 61].

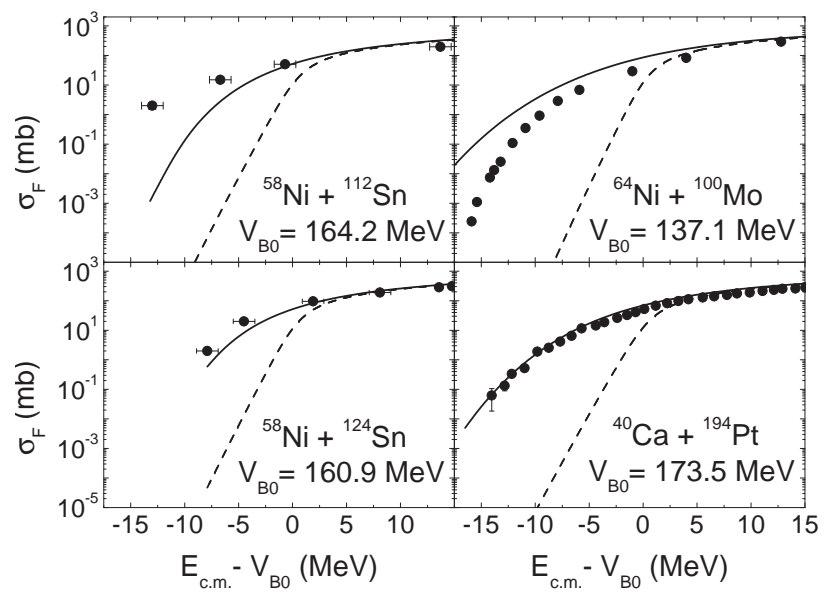

(b) Os dados são das Refs. [11, 90, 95, 96].

Figura 5.6: Dados de fusão e correspondentes seções de choque teóricas para BPM não-deformado (linhas tracejadas) e para o modelo de ZPM (linhas cheias) para os sistemas indicados nas figuras. As alturas das barreiras dos potenciais não-deformados para as ondas s são apresentadas na figura. No caso dos sistemas ${ }^{58} \mathrm{Ni}+{ }^{112,124} \mathrm{Sn}$, incertezas experimentais de $1 \mathrm{MeV}$ nos correspondentes valores de energia são indicados na figura.

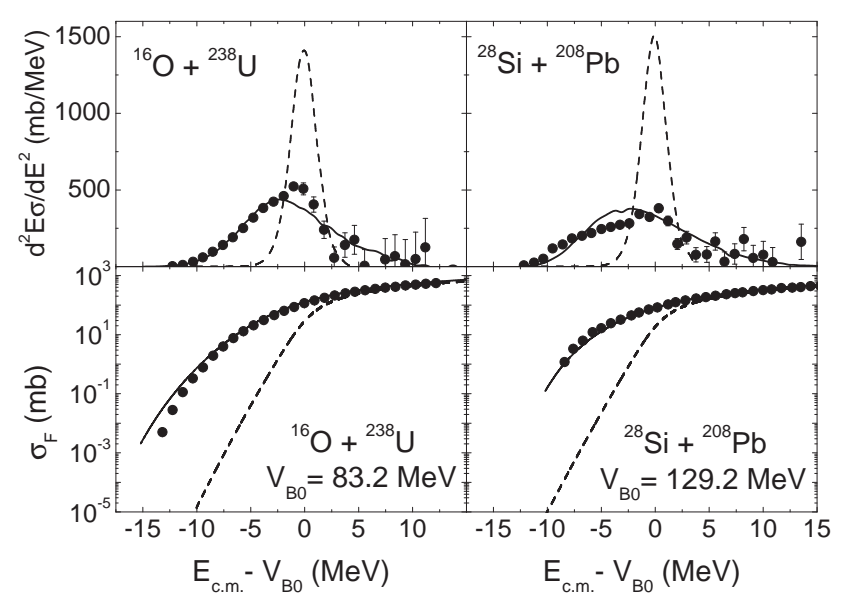

(a) Os dados são das Refs. [97, 98].

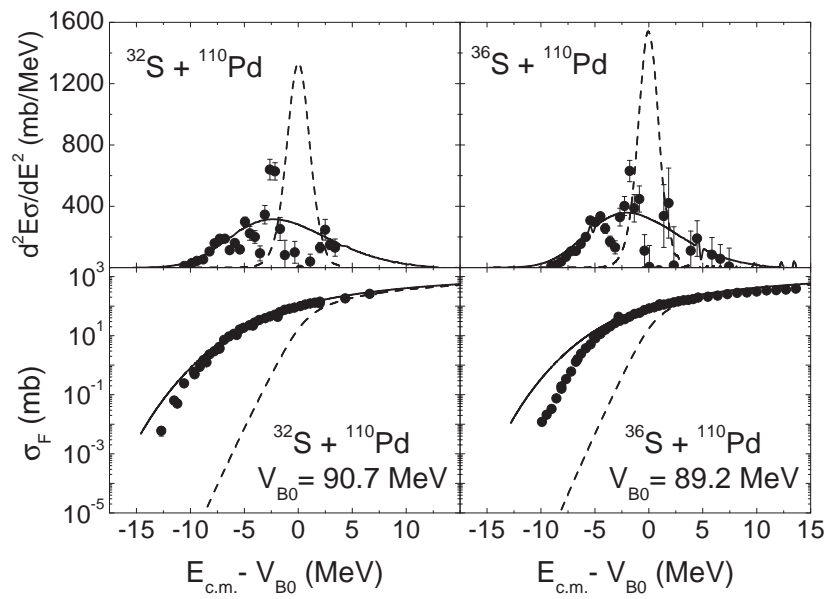

(b) Os dados são das Refs. [86, 99].

Figura 5.7: Dados de seção de choque de fusão (embaixo) e correspondentes distribuições de barreira (em cima) para os sistemas ${ }^{16} \mathrm{O}+{ }^{238} U,{ }^{28} \mathrm{Si}+{ }^{208} \mathrm{~Pb} e{ }^{32,36} \mathrm{~S}+{ }^{110} \mathrm{Pd}$. As linhas tracejadas e cheias correspondem às seções de choque de BPM e ZPM, respectivamente. 


\subsection{Discussão dos Resultados do Modelo de ZPM}

Na Figura 5.8, mostramos um resumo dos resultados apresentados neste capítulo, calculando a razão entre dados de fusão e as correspondentes seções de choque de BPM ou ZPM. As exceções são os sistemas ${ }^{84} \mathrm{Kr}+{ }^{104} \mathrm{Ru}$ e ${ }^{90} \mathrm{Zr}+{ }^{90,96} \mathrm{Zr}$ para os quais os dados não correspondem à fusão total mesmo em energias abaixo da barreira. Incluímos também nesta análise os resultados de ZPM para o sistema ${ }^{64} \mathrm{Ni}+{ }^{64} \mathrm{Ni}$, expressos na Figura 4.2 (página 55, no Capítulo 4). Em energias bem abaixo da barreira, os dados são subestimados pelos cálculos de BPM por onze ordens de magnitude (ver Figura 5.8). Por outro lado, quase todas seções de choque de ZPM concordam com os dados dentro de apenas duas ordens de grandeza (ver Figura 5.8). Logo, é razoável considerar que os acoplamentos a estados excitados inelásticos são responsáveis pela maior parte dos enhancements dos dados em relação ao BPM. Além do conjunto de dados apresentado neste capítulo, analisamos também dados de aproximadamente outros 30 sistemas. Os resultados correspondentes são bastante similares àqueles aqui apresentados, i.e. as previsões do modelo de ZPM concordam com a maioria dos dados dentro de duas ordens de magnitude.
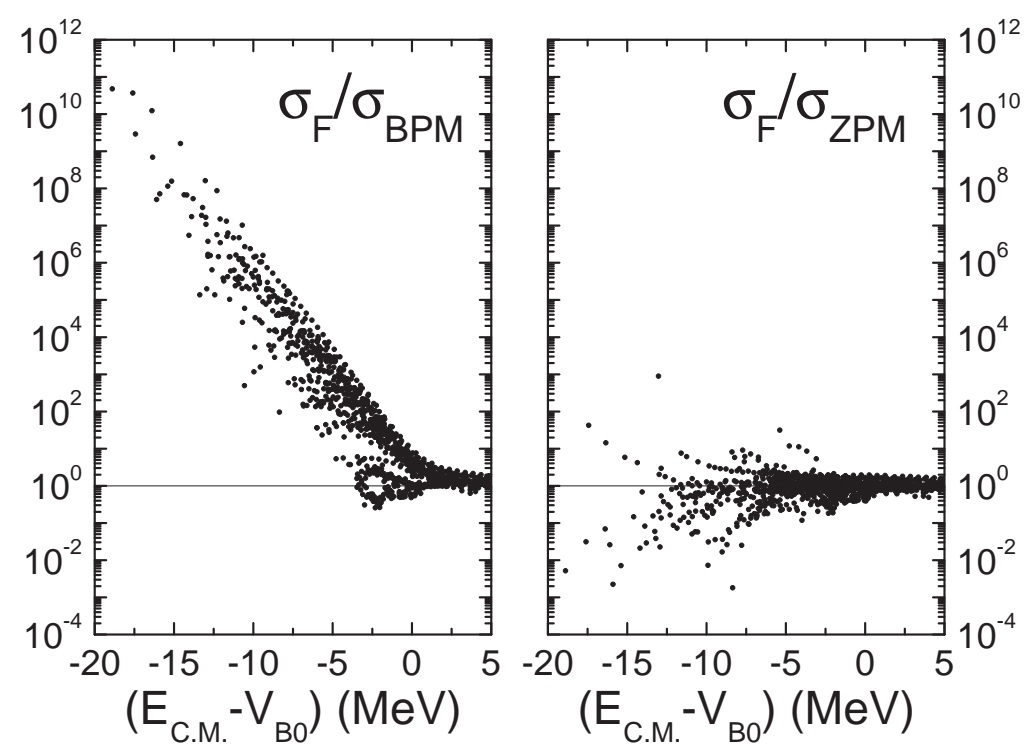

Figura 5.8: Razões entre dados de fusão e seções de choque teóricas de BPM (lado esquerdo) e entre os dados e cálculos do modelo de ZPM (lado direito) para os sistemas apresentados nas Figuras 5.1 a 5.7 e na Figura 4.2 (página 55).

Como comentado na Seção 5.1, apesar de alguns núcleos envolvidos em nossa análise serem 
melhor representados pelo modelo rotacional, assumimos o modelo de ZPM para calcular a seção de choque de fusão. Mesmo assim, concordância similar entre os dados e as previsões teóricas foi obtida para todos os sistemas. Por exemplo, o ${ }^{144} \mathrm{Sm}$ é considerado um vibrador enquanto o ${ }^{154} \mathrm{Sm}$ deveria ser melhor representado como um rotor. No entanto, os resultados obtidos para os sistemas ${ }^{16} \mathrm{O},{ }^{40} \mathrm{Ar}+{ }^{154} \mathrm{Sm}$ (Figuras 5.2(c) e 5.3(f)) são tão bons quanto aqueles obtidos para os sistemas ${ }^{16} \mathrm{O}$, ${ }^{28} \mathrm{Si},{ }^{40} \mathrm{Ar}+{ }^{144} \mathrm{Sm}$ (Figuras 5.2(c), 5.2(d) e 5.4). Outros exemplos são os núcleos ${ }^{238} \mathrm{U}$ (rotacional) e ${ }^{58,64} \mathrm{Ni}$ (vibracionais), para os quais os correspondentes resultados obtidos para os sistemas ${ }^{16} \mathrm{O}$ $+{ }^{238} \mathrm{U}$ (Figura 5.7(a)) e ${ }^{58,64} \mathrm{Ni}+{ }^{58,64} \mathrm{Ni}$ (Figuras 5.6(a) e 4.2, página 55) são bastante similares. Neste sentido, o modelo de ZPM se mostra apropriado para descrever a fusão também para sistemas envolvendo núcleos rotacionais. Como já comentado, esta característica se deve provavelmente ao fato de que a Eq. (4.8) fornece o resultado exato para desvios padrão de ambos os modelos vibracional e rotacional.

Na Figura 5.9, apresentamos os dados de fusão para os sistemas ${ }^{40} \mathrm{Ca},{ }^{40} \mathrm{Ar}+{ }^{116} \mathrm{Sn}$. Os resultados de BPM e ZPM correspondem às linhas tracejadas e cheias, respectivamente. Enquanto para o projétil ${ }^{40} \mathrm{Ar}$ as seções de choque de ZPM estão em bom acordo com os dados, para o ${ }^{40} \mathrm{Ca}$ os dados abaixo da barreira estão em torno de três ordens de magnitude maiores que as previsões de ZPM. Este é um resultado surpreendente visto que ambos os sistemas são na verdade bastante similares (mesmo alvo e também mesma massa do projétil). Investigamos, como segue, se esta diferença poderia ser reduzida introduzindo modificações em alguns parâmetros envolvidos nos cálculos teóricos.

Como comentado anteriormente, as deformações nucleares e coulombianas deveriam apresentar valores similares, mas não necessariamente os mesmos, como assumido nos cálculos de ZPM. Por outro lado, os valores de $\beta$ foram obtidos de amplitudes de transição (Eq. (3.7)), que são aquelas encontradas nas Refs. [51, 65]. As amplitudes de transição experimentais possuem, obviamente, incertezas experimentais. Por exemplo, o dado experimental de $B(E 2)$ para o ${ }^{40} \mathrm{Ca}$ possui uma incerteza em torno de $16 \%$ [65], o que resulta em uma incerteza de aproximadamente $8 \%$ no correspondente valor de $\beta_{2}$. Logo, há espaço para pequenas modificações nos valores de $\beta$ nos cálculos de ZPM. Para ajustar os dados para o sistema ${ }^{40} \mathrm{Ca}+{ }^{116} \mathrm{Sn}$, incluímos em nossa análise um fator ajustável (o mesmo para alvo e projétil) que renormaliza os valores de $\beta$ apresentados na Tabela 5.1. A linha pontilhada na Figura 5.9 representa os resultados correspondentes. Uma concordância 


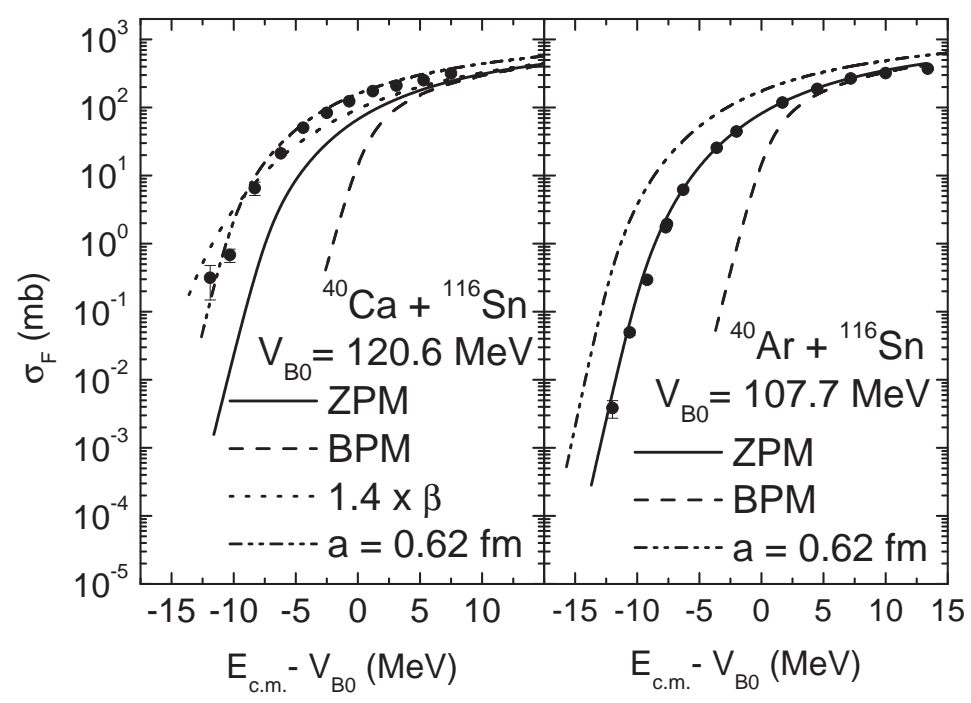

Dados experimentais obtidos das Refs. [92, 100].

Figura 5.9: Dados de seção de choque de fusão para os sistemas ${ }^{40} \mathrm{Ca},{ }^{40} \mathrm{Ar}+{ }^{116} \mathrm{Sn}$. As linhas tracejadas e cheias correspondem às seções de choque de BPM e ZPM (padrão), respectivamente. As outras linhas correspondem a cálculos de ZPM onde os valores de $\beta$ ou da difusividade das densidades a foram modificados (ver texto para detalhes).

muito melhor entre dados e seções de choque teóricas é agora obtida. No entanto, um fator grande, e provavelmente não-realista, de 1.4 é necessário para renormalizar os valores de $\beta$. Evidentemente, esta diferença seria ainda mais significativa se o fator tivesse sido aplicado apenas em relação ao valor de $\beta$ do ${ }^{40} \mathrm{Ca}$ (os resultados para o sistema ${ }^{40} \mathrm{Ar}+{ }^{116} \mathrm{Sn}$ indicam que o valor de $\beta$ para o ${ }^{116} \mathrm{Sn}$ não deveria ser renormalizado).

Uma outra possibilidade para explicar a diferença entre o comportamento dos sistemas ${ }^{40} \mathrm{Ar}+$ ${ }^{116} \mathrm{Sn}$ e ${ }^{40} \mathrm{Ca}+{ }^{116} \mathrm{Sn}$ está relacionada ao potencial de São Paulo (SP), utilizado como potencial bare em todos os cálculos. No presente capítulo, assumimos o potencial de SP no contexto da sistemática para as densidades nucleares (Seção 3.1). Esta sistemática foi baseada [19] em cálculos teóricos com o modelo de Dirac-Hartree-Bogoliubov e também em resultados experimentais para distribuições de carga. Como assumido aqui, considerando a sistemática de densidades, os raios das distribuições biparamétricas de Fermi $(2 \mathrm{pF})$ são bem representadas pela Eq. (3.6), com um valor de difusividade de $a=0.56 \mathrm{fm}$. No entanto, estes valores de raio e difusividade representam um comportamento médio obtido considerando os resultado para um grande número de núcleos. Logicamente, pequenos 
desvios em torno desses valores médios são esperados devido aos efeitos de estrutura dos núcleos (vide Figuras 3.1 e 3.2, página 28). Na Ref. [19], estas variações foram analisadas e desvios-padrão de $\sigma_{R}=0.07 \mathrm{fm}$ e $\sigma_{a}=0.025 \mathrm{fm}$, relativos aos valores médios de raio e difusividade, respectivamente, foram encontrados. Logo, é possível tratar $R_{0}$ e $a$ como parâmetros ajustáveis. Na Ref. [19] foi demonstrado que a altura da barreira é mais afetada por variações no valor da difusividade do que no do raio. Logo, para o sistema ${ }^{40} \mathrm{Ca}+{ }^{116} \mathrm{Sn}$ assumimos a difusividade das densidades (mesmo valor para projétil e alvo) como um parâmetro livre para ajustar os dados. O resultado correspondente é mostrado como linhas traço-ponto duplo na Figura 5.9. Uma boa descrição dos dados é agora obtida com $a=0.62 \mathrm{fm}$. Em um primeiro momento, esta difusividade aparenta ser razoável. Porém, este valor difere da difusividade média $(a=0.56 \mathrm{fm})$ por mais de dois desvios-padrão $\sigma_{a}=0.025 \mathrm{fm}$. Esta diferença é ainda mais significativa pois foi aplicada tanto ao projétil como ao núcleo-alvo. Esta aparentemente pequena modificação do valor da difusividade de $0.56 \mathrm{fm}$ para $0.62 \mathrm{fm}$ representa, na verdade, uma grande variação de aproximadamente 50\% na intensidade do potencial nuclear nãodeformado no raio da barreira de onda $s$. Na Figura 5.10 apresentamos os resultados teóricos de Dirac-Hartree-Bogoliubov para as densidades de matéria dos núcleos ${ }^{40} \mathrm{Ar},{ }^{40} \mathrm{Ca}{ }^{116} \mathrm{Sn}$ (ver símbolos na figura). Na mesma figura, distribuições $2 \mathrm{pF}$ com diferentes valores de difusividades também são mostradas pelas linhas cheias e tracejadas. Como esperado, o valor da difusividade média obtido na sistemática [19], $a=0.56 \mathrm{fm}$, representa um resultado muito melhor para as densidades dos três núcleos do que $a=0.62 \mathrm{fm}$. Além do mais, a densidade teórica do núcleo ${ }^{40} \mathrm{Ca}$ é muito similar àquela para $\mathrm{o}{ }^{40} \mathrm{Ar}$. No entanto, se considerarmos o mesmo $a=0.62 \mathrm{fm}$, obtido do ajuste de dados de fusão para o ${ }^{40} \mathrm{Ca}$, também para o sistema ${ }^{40} \mathrm{Ar}+{ }^{116} \mathrm{Sn}$, isto resulta em grande discordância entre os dados de fusão e cálculos de ZPM (ver Figura 5.9). Enfatizamos o fato de que os dados de fusão para o sistema ${ }^{40} \mathrm{Ca}+{ }^{116} \mathrm{Sn}$ também poderiam ser bem ajustados considerando variações nos raios, ao invés da difusividade, das densidades. Neste caso, encontramos uma variação de $\Delta R_{0} \approx 0.24 \mathrm{fm}$, que é também não-realista visto que o desvio-padrão para os raios nucleares é apenas de $\sigma_{R}=0.07$ fm.

Obviamente, uma concordância muito melhor entre os dados de fusão e cálculos de ZPM poderia ser obtida para todos os sistemas apresentados neste capítulo se tivéssemos assumido a e/ou $\beta$ como sendo parâmetros ajustáveis. Porém, este procedimento é apropriado somente quando os valores re- 


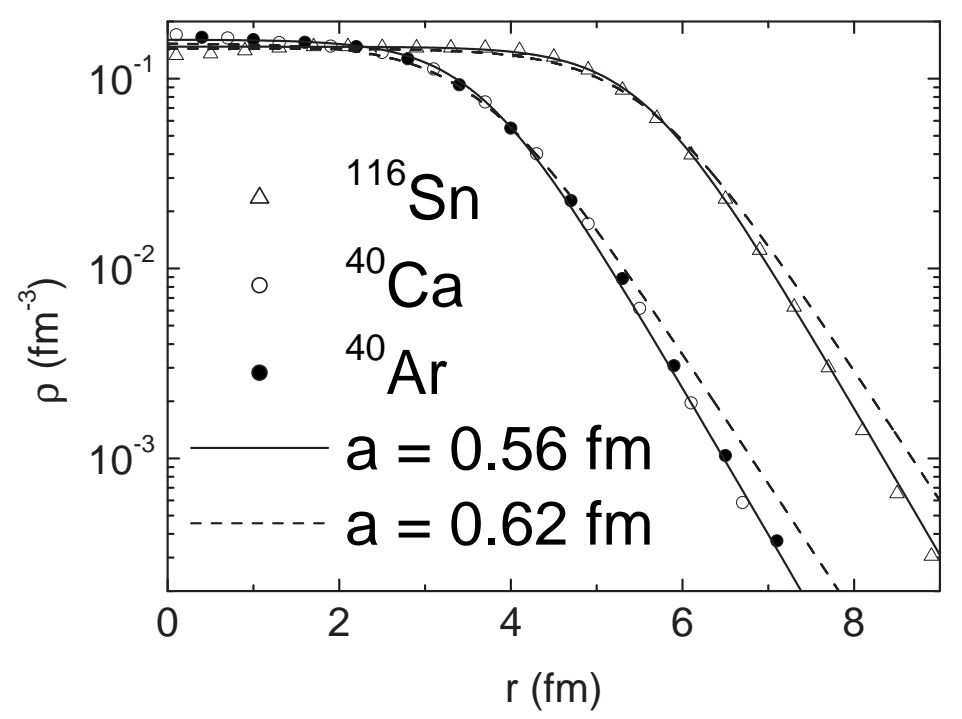

Figura 5.10: Os símbolos representam densidades de matéria teóricas calculadas através do modelo de Dirac-Hartree-Bogoliubov. As linhas cheias e tracejadas correspondem a distribuições $2 \mathrm{pF}$ com diferentes valores de difusividades. Os raios das distribuições $2 p F$ foram obtidos pela Eq. (3.6).

sultantes dos parâmetros estão dentro de certas regiões aceitáveis. Este é um assunto importante visto que em muitos trabalhos encontrados na literatura o potencial bare contém parâmetros ajustáveis. Neste capítulo, demonstramos que uma aparentemente pequena variação de $0.06 \mathrm{fm}$ no valor da difusividade (ou 0.24 fm no raio) na verdade não é realista e pode esconder possíveis outras características importantes do sistema. Enfatizamos que tal conclusão é muito mais fácil de ser obtida se as análises de dados forem realizadas sem o uso de qualquer parâmetro ajustável. As discrepâncias entre os sistemas similares ${ }^{40} \mathrm{Ar}+{ }^{116} \mathrm{Sn} \mathrm{e}{ }^{40} \mathrm{Ca}+{ }^{116} \mathrm{Sn}$ estão provavelmente relacionadas a algum efeito de estrutura. Por exemplo, acoplamentos a canais de transferência podem resultar em diferentes contribuições para o processo de fusão.

Nos voltamos agora ao problema de energias extremamente abaixo da barreira que é discutido em muitos trabalhos recentes (e.g. [6-10]). Em alguns trabalhos, o conceito de energias extremamente abaixo da barreira foi relacionado à seções de choque muito pequenas. Por exemplo, no caso do sistema ${ }^{64} \mathrm{Ni}+{ }^{64} \mathrm{Ni}[8]$ o dado de menor de energia $\left(E \approx V_{B 0}-10 \mathrm{MeV}\right.$ - ver Figura 4.2, página 55) corresponde a um valor de seção de choque em torno de $10^{-5} \mathrm{mb}$. No entanto, muitos sistemas aqui estudados possuem seções de choque medidas em energias ainda menores (em relação à altura da barreira), com valores de seções de choque significativamente maiores. Por exemplo, para $E \approx$ 
$V_{B 0}-17.5 \mathrm{MeV}$, no caso do sistema ${ }^{40} \mathrm{Ca}+{ }^{192} \mathrm{Os}$ (Figura 5.3(d)), pode-ser observar uma seção de choque medida de em torno de $10^{-1} \mathrm{mb}$. Claramente, neste caso a seção de choque é muito maior do que aquela para o sistema ${ }^{64} \mathrm{Ni}+{ }^{64} \mathrm{Ni}$ pois o enhancement correspondente devido aos acoplamentos inelásticos é maior. Logo, relacionamos o termo "energia extremamente abaixo da barreira" a baixos valores de energia relativos a altura da barreira, ao invés de pequenos valores de seções de choque.

Em alguns trabalhos, foi observado que os valores de difusividade para potenciais ajustados para reproduzir dados de fusão são significativamente maiores do que aqueles usualmente assumidos em análises de dados de espalhamento elástico. No entanto, em nosso trabalho a interação nuclear assumida nos presentes cálculos de ZPM para a fusão, o potencial de SP, também foi aplicada com sucesso, de maneira consistente, ao processo de espalhamento elástico. Também foi relatado que os dados de fusão em energias extremamente abaixo da barreira não foram bem descritos por cálculos de CC. Por exemplo, no caso do sistema ${ }^{64} \mathrm{Ni}+{ }^{64} \mathrm{Ni}$, cálculos detalhados de CC foram feitos usando parâmetros ajustáveis no potencial bare para descrever os dados de fusão em alta energia [8]. Porém, em energias muito abaixo da barreira, os dados exibem uma queda muito mais abrupta em comparação com os resultados teóricos. Os presentes cálculos de CC reforçam este fato (ver Figura 4.2 , página 55).

Como já comentado, para a maioria dos sistemas aqui estudados, as previsões de ZPM reproduzem os dados dentro de duas ordens de magnitude. Na Figura 5.9 pode-se observar maiores enhancements em relação ao BPM para energias mais baixas (painel da esquerda). Visto que estes enhancements advêm dos efeitos dos acoplamentos, uma dispersão maior dos dados em relação às seções de choque de ZPM (painel da direita) é esperada para energias mais baixas. Porém, nenhuma mudança brusca aparenta ser detectada no comportamento médio da seção de choque de fusão ao aproximarmo-nos de energias extremamente abaixo da barreira. Mesmo assim, um cálculo mais quantitativo pode modificar este quadro. Para investigar este ponto, calculamos valores médios para a ordem de grandeza dos desvios entre os dados de fusão e as seções de choque de ZPM. Dividimos os dados apresentados na Figura 5.9 (painel da direita) em passos consecutivos de $2 \mathrm{MeV}$. Para cada passo, calculamos o valor médio de $\ln \left(\sigma_{F} / \sigma_{Z P M}\right)$, e definimos valores médios para a ordem de magnitude através da seguinte expressão $\left\langle\sigma_{F} / \sigma_{Z P M}\right\rangle=\mathrm{e}^{\left\langle\ln \left(\sigma_{F} / \sigma_{Z P M}\right)\right\rangle}$. Os correspondentes resultados são apresentados na Figura 5.11. Desde energias acima da barreira até aproximadamente $6 \mathrm{MeV}$ abaixo da barreira, em 
média as previsões de ZPM concordam com os dados dentro de 10\% de precisão (ver Figura 5.11). Para $-15 \leq E-V_{B 0} \leq-7 \mathrm{MeV}$, em média o modelo de ZPM superestima ligeiramente a seção de choque medida por um fator em torno de dois. Em energias extremamente abaixo da barreira, os dados são aproximadamente uma ordem de magnitude menores do que as previsões teóricas. Evidentemente, estes resultados para valores médios deveriam ser considerados em um sentido restrito pois eles se referem apenas ao particular conjunto de dados aqui analisados. Veremos, no Capítulo 7, que a extensão desses cálculos a um conjunto maior de sistemas modifica um pouco os resultados apresentados na Figura 5.11.

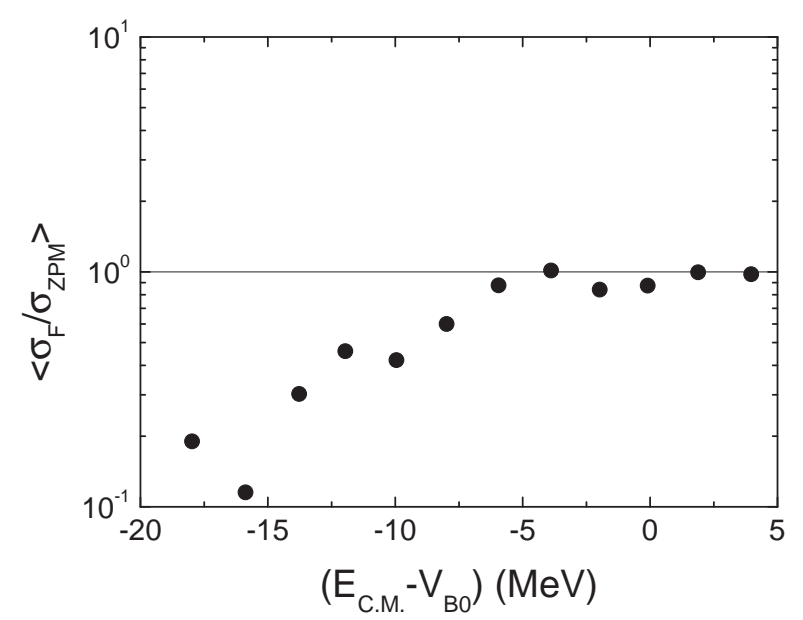

Figura 5.11: Valores médios das ordens de magnitude dos desvios entre os dados de fusão e as seções de choque de ZPM (ver texto para detalhes). 


\section{Capítulo 6}

\section{Modelo para o Potencial de Polarização}

Voltamo-nos agora para o processo de espalhamento elástico. A Figura 6.1 (embaixo) mostra o potencial efetivo para a onda $s, V_{\text {ef }}$, para duas diferentes configurações dos parâmetros de deformação. As linhas pontilhadas na figura representam parábolas que resultam em uma mesma altura e curvatura de barreira que $V_{\text {ef }}$. Como poderia-se esperar, as parábolas são boas representações de $V_{\text {ef }}$ apenas em uma região limitada em torno do raio da barreira. Logo, a aplicação do modelo de ZPM, como desenvolvido na Seção 2.4, não deveria descrever tão bem o processo de espalhamento elástico de íons pesados, visto que este é sensível a uma estendida região superficial de interação. Além disso, nesta região estendida, a intensidade do acoplamento não pode ser aproximada por um valor constante. Por exemplo, na Figura 6.1 (em cima) mostramos a variação, $F_{\lambda i}(R)$, de $V_{\text {ef }}$ como uma função da distância entre os núcleos, no caso de $\lambda=2$ e $i=1\left({ }^{58} \mathrm{Ni}\right)$ :

$$
F_{\lambda i}(R)=-\frac{\partial V_{\mathrm{ef}}(R)}{\partial s_{\lambda i}}
$$

Em particular, existe um ponto (indicado por uma seta na Figura 6.1) a partir do qual $F(R)$ se torna negativo, pois o potencial nuclear é desprezível para grandes distâncias e o acoplamento coulombiano se torna a contribuição principal para $F(R)$. Outro ponto importante é que a "cauda" de longo alcance do potencial coulombiano é incompatível com o comportamento assintótico de onda livre de $\bar{Y}$, como assumido na Seção 2.4. Mesmo assim, acreditamos que uma abordagem similar àquela desenvolvida na Seção 2.4 deveria funcionar em uma pequena região em torno do raio da barreira, 
onde $V_{\text {ef }}$ é aproximadamente parabólico. Logo, propomos o seguinte método para obter o potencial de polarização.

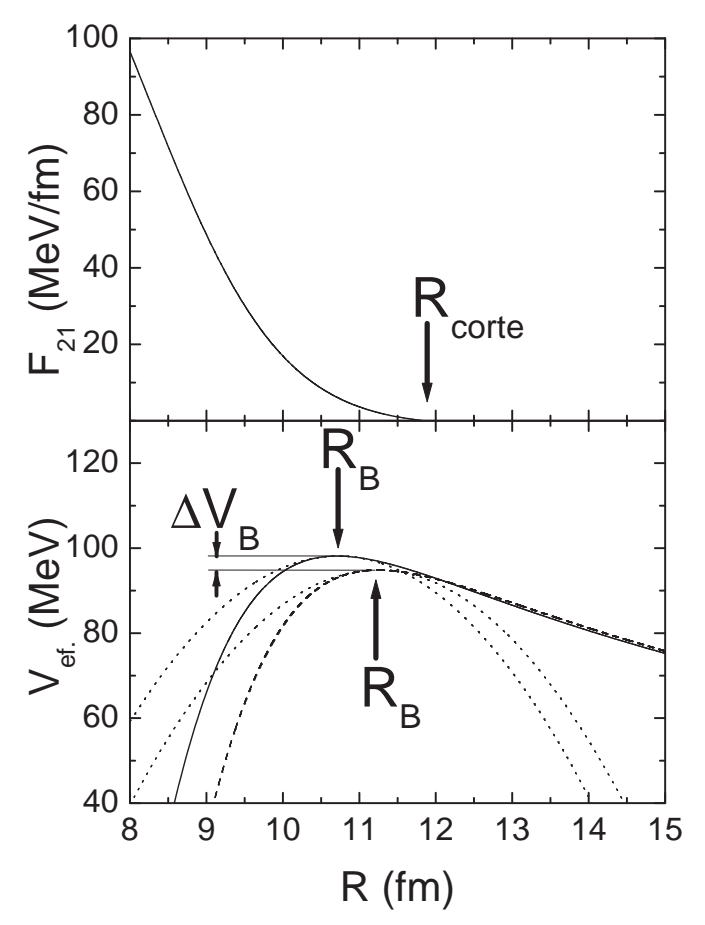

Figura 6.1: EMBAIXO: Potencial efetivo para a onda s para potenciais não-deformado (linha cheia) $e$ deformado (linha tracejada) para o sistema ${ }^{58} \mathrm{Ni}+{ }^{64} \mathrm{Ni}$. A deformação corresponde apenas ao modo $\lambda=2 d{ }^{58} \mathrm{Ni}$, com $\beta_{21}=0.215$. As linhas pontilhadas representam barreiras parabólicas que resultam em uma mesma altura e curvatura de barreira do potencial efetivo. As setas indicam os correspondentes raios de barreira. A variação da altura da barreira também é indicada na figura. EM CIMA: Intensidade de acoplamento no caso de $\lambda=2$ e $i=1\left({ }^{58} \mathrm{Ni}\right)$ como calculado pela Eq. (6.1). A seta na figura indica o ponto $R_{\text {corte }}$ onde $F_{21}=0$.

Inicialmente, truncamos $F_{\lambda i}(R)$ no ponto (que chamamos $R_{\text {corte }}$ ) onde $F_{\lambda i}(R)$ se torna negativo (ver seta na Figura 6.1 - em cima), e assumimos $F_{\lambda i}\left(R \geq R_{\text {corte }}\right)=0$. Baseado na Eq. (2.66), propomos calcular $\bar{Y}$ através:

$$
\left[-\frac{\hbar^{2}}{2 \mu} \frac{\partial^{2}}{\partial r^{2}}+V_{\mathrm{ef}}(R)+i W(R)+\sum_{\lambda i} F_{\lambda i}(R) \sigma_{\lambda i}\left(\frac{n_{\lambda i}-\left\langle m_{\lambda i}\right\rangle}{\sigma_{m_{\lambda i}}}\right)\right] \bar{Y}=E \bar{Y}
$$

onde $V_{\text {ef }}(R)$ é calculado com o potencial não-deformado, $W(R)$ é um potencial imaginário interno para absorver o fluxo, e $\bar{Y}$ tem o comportamento assintótico coulombiano usual para o espalhamento elástico. O potencial de polarização é então calculado a partir da Eq. (2.63), com $F_{\lambda i}(R)$ ao invés de 
$F_{i}$. No cálculo dos pesos, termos $U_{m n},\langle m\rangle$ e $\sigma_{m}$, usamos os valores constantes de $F$ como obtidos pela Eq. (4.7). Obviamente, este método é inspirado pelos resultados obtidos na Seção 2.4 e deveria, portanto, fornecer estimativas razoáveis para a polarização apenas em uma região em torno do raio

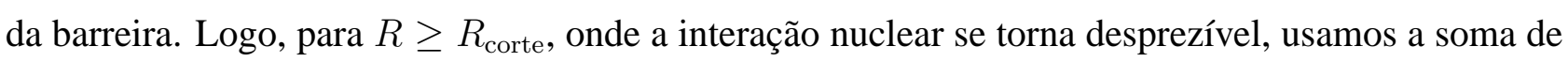
diferentes modos de vibração da parte imaginária da polarização de excitação coulombiana pura, que foi anteriormente obtida sob a forma de expressões analíticas [101-103]. No Apêndice E mostramos as expressões usadas como componente coulombiana do potencial de polarização que incorporam efeitos dos acoplamentos aos estados de um fônon $2^{+}$(quadrupolo) e $3^{-}$(octupolo), levando em consideração as energias de excitação finitas dos núcleos.

Na Figura 6.2, apresentamos os dados de espalhamento elástico, resultados do potencial bare, previsões do modelo de ZPM e cálculos de CC considerando apenas acoplamentos coulombianos ou coulombianos mais nucleares. Os cálculos de CC foram feitos considerando apenas os estados $2^{+} \mathrm{e}$ $3^{-}$. Claramente, o modelo de ZPM fornece previsões bastante razoáveis para as seções de choque elásticas para todas as energias apresentadas. Os cálculos de CC não fornecem os mesmos resultados que aqueles obtidos pelo modelo de ZPM. No entanto, como já discutimos anteriormente, cálculos de ZPM e de CC são diferentes modelos para um problema complexo e, conseqüentemente, não se deve esperar que ambos dêem as mesmas previsões teóricas.

A Figura 6.3 apresenta a matriz S correspondente para as quatro distribuições angulares. Podese ver que os cálculos de ZPM e de CC fornecem valores similares de momento angular de onda grazing, $\ell_{g}$, que definimos como $1-\left|S\left(\ell_{g}\right)\right|^{2} \approx 0.5$ ou $\left|S\left(\ell_{g}\right)\right| \approx 0.7$. O potencial de polarização é dependente de $\ell$. Na Figuras 6.4 apresentamos as partes reais e imaginárias, respectivamente, do potencial de polarização para $\ell_{g}$, o que corresponde aproximadamente a $\ell_{g} \approx 10,30,45$ e 60 para $E_{c . m .}=95.6,99.5,106.5$ e $115 \mathrm{MeV}$, respectivamente. As setas nesta figura representam as posições dos raios das barreiras de onda $\ell$ do potencial não-deformado. Em relação à parte real do potencial de polarização (Figura 6.4(a)), os cálculos de ZPM e de CC fornecem resultados de mesma ordem de magnitude. No entanto, para a parte imaginária (Figura 6.4(b)), o ZPM prevê intensidades muito maiores em comparação com os cálculos de CC na região $R<R_{B}$. Mesmo assim, isto não deve ser um problema importante pois, a medida em que o sistema tunela através do raio da barreira, o fluxo deve ser absorvido pelo potencial imaginário interno. A Figura 6.5 mostra o potencial de polarização 


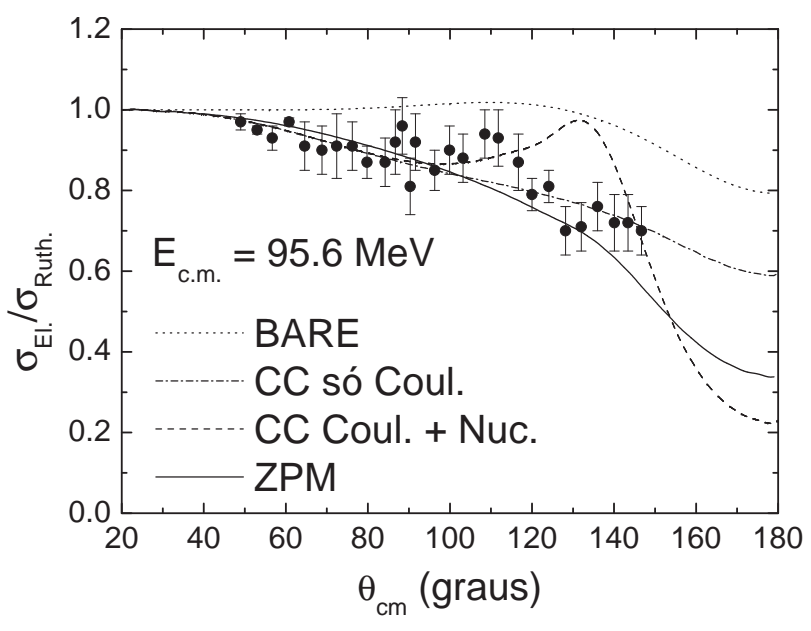

(a) $E_{c . m .}=95.6 \mathrm{MeV}$.

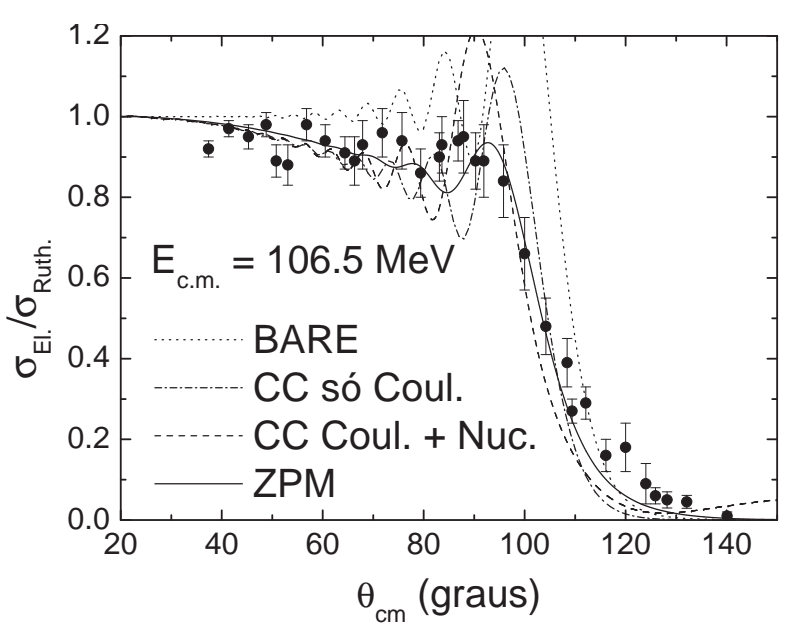

(c) $E_{c . m .}=106.5 \mathrm{MeV}$.

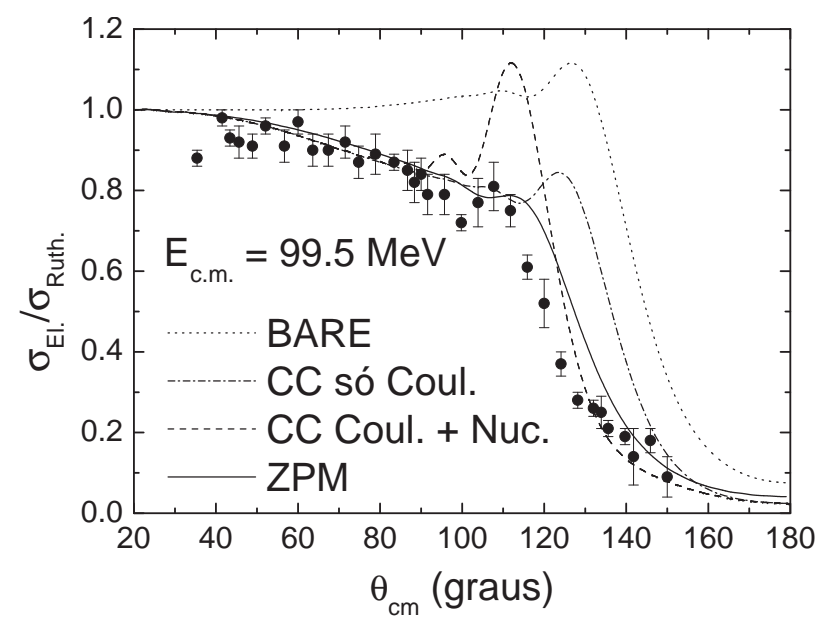

(b) $E_{c . m .}=99.5 \mathrm{MeV}$.

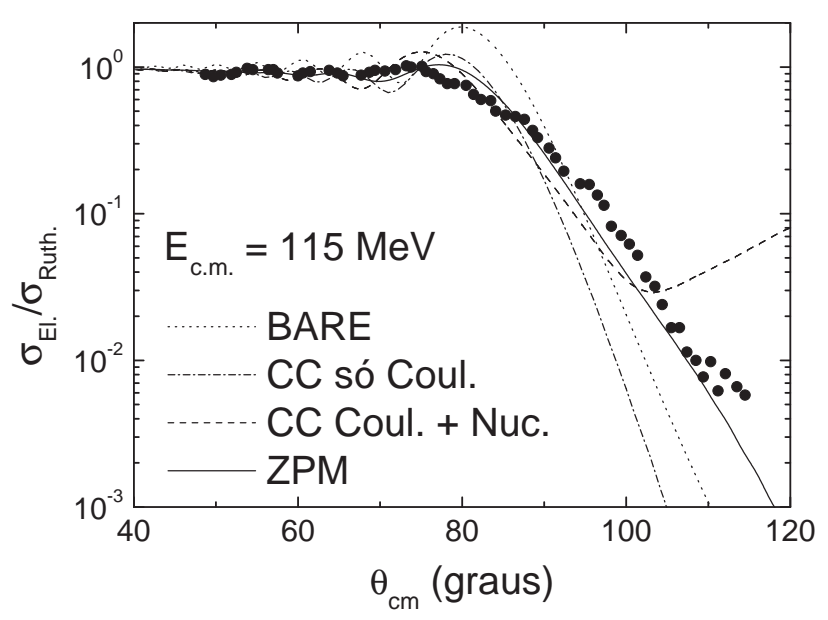

(d) $E_{c . m .}=115 \mathrm{MeV}$.

Figura 6.2: Distribuições angulares de espalhamento elástico para o sistema ${ }^{58} \mathrm{Ni}+{ }^{64} \mathrm{Ni}$ nas energias $E_{c . m .}=95.6,99.5,106.5$ e $115 \mathrm{MeV}$. As linhas correspondem a diferentes cálculos teóricos (ver texto para detalhes). 


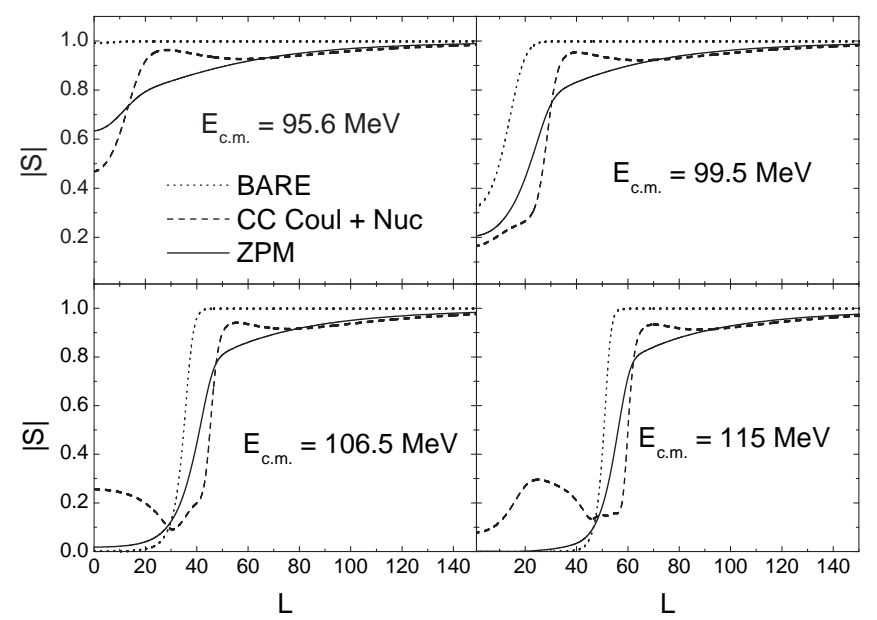

Figura 6.3: Módulo da matriz S para as quatro distribuições angulares de espalhamento elástico analisadas no presente capítulo.

para $E_{c . m .}=106.5 \mathrm{MeV}$ para uma onda parcial menor $(\ell=30)$ e maior $(\ell=60)$ que valor de grazing correspondente $\left(\ell_{g} \approx 45\right)$. Os resultados são claramente similares àqueles obtidos para $\ell_{g}$.

Apresentamos também os dados experimentais e seções de choque teóricas previstas pelo modelo de ZPM para o espalhamento elástico para os sistemas ${ }^{16} \mathrm{O}+{ }^{58} \mathrm{Ni},{ }^{208} \mathrm{~Pb}$ nas Figuras 6.6 e 6.7. Podemos ver novamente que, levando-se em consideração a total ausência de parâmetros livres e as aproximações assumidas no modelo de ZPM, as previsões teóricas se aproximam de forma razoável aos dados experimentais.

Inspirados no modelo unidimensional proposto na Seção 2.4, propusemos um método, o modelo de ZPM, para obtermos o potencial de polarização para sistemas de íons pesados. Neste capítulo ele é apresentado e aplicado com razoável sucesso à descrição dos dados experimentais de espalhamento elástico para os sistemas ${ }^{58} \mathrm{Ni}+{ }^{64} \mathrm{Ni} \mathrm{e}{ }^{16} \mathrm{O}+{ }^{58} \mathrm{Ni},{ }^{208} \mathrm{~Pb}$ em energias em torno da barreira. $\mathrm{O}$ modelo de ZPM também foi anteriormente aplicado com bastante sucesso na descrição do processo de fusão para uma grande variedade de sistemas [36], como mostram os Capítulos 5 e 7. O modelo de ZPM produz resultados aparentemente mais satisfatórios para a fusão do que para o processo de espalhamento elástico. Isso se deve ao fato de que as intensidades de acoplamento (fatores de forma) na realidade variam com a distância entre os núcleos e o espalhamento elástico é bastante sensível a uma região em torno do raio da barreira, ao passo que a fusão depende muito mais simplesmente da 


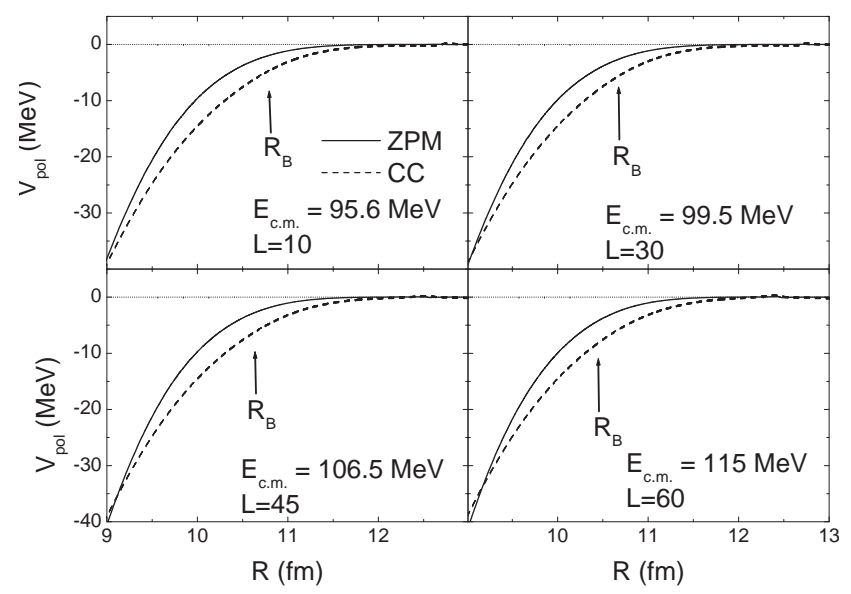

(a) Parte real do potencial de polarização.

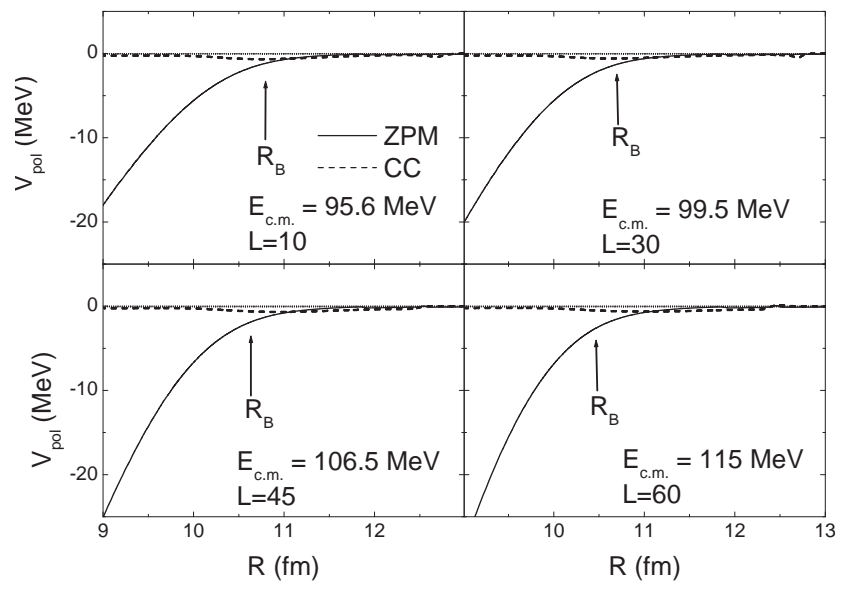

(b) Parte imaginária do potencial de polarização.

Figura 6.4: Partes real e imaginária do potencial de polarização para a onda parcial grazing $L=\ell_{g}$ para as quatro distribuições angulares analisadas na Figura 6.2. As linhas cheias e tracejadas na figura representam os resultados de cálculos de ZPM e de CC respectivamente. Foram considerados nos cálculos de CC os acoplamentos nuclear e coulombiano aos estados $2^{+} e 3^{-}$de ambos os núcleos. As setas na figura representam a posição do raio da barreira da onda $\ell_{g}$ do potencial não-deformado. As linhas pontilhadas marcam o nivel $V_{\mathrm{pol}}=0$.

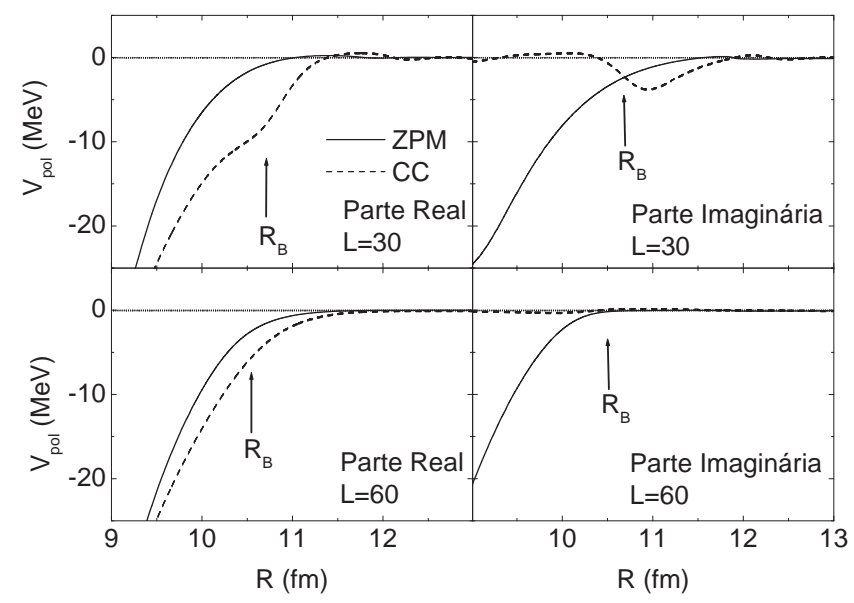

Figura 6.5: Partes real e imaginária do potencial de polarização para $\ell=30$ e 60 na energia de $E_{c . m .}=106.5 \mathrm{MeV}$. As linhas pontilhadas marcam o nivel $V_{\mathrm{pol}}=0$. 

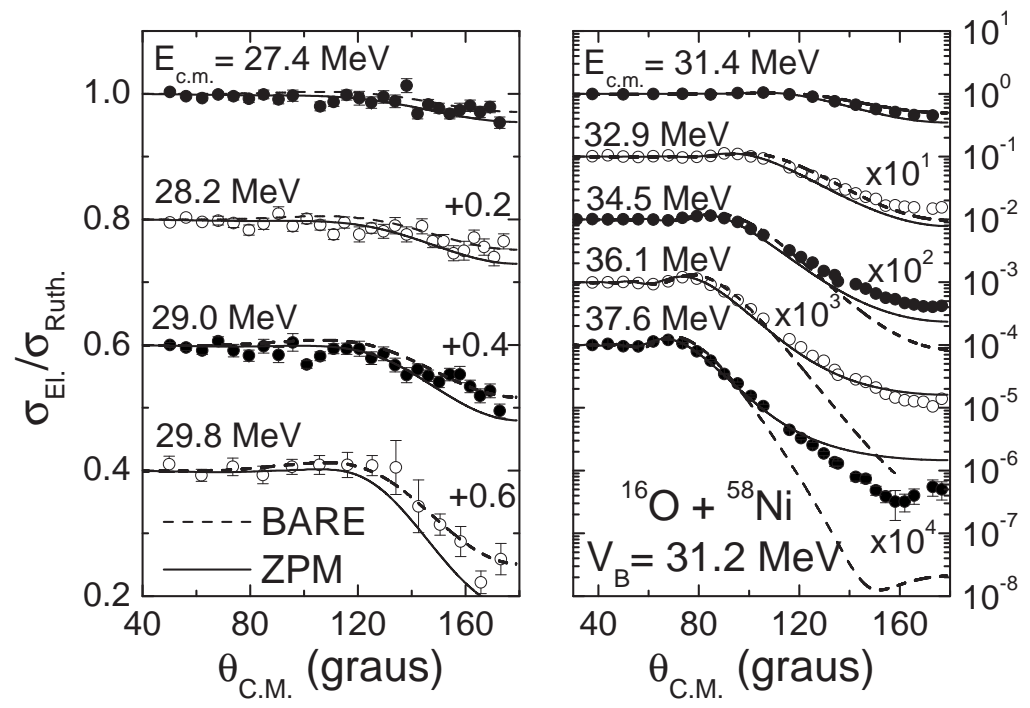

Figura 6.6: Distribuições angulares de espalhamento elástico para o sistema ${ }^{16} \mathrm{O}+{ }^{58} \mathrm{Ni}$ em diversas energias. As linhas tracejadas correspondem aos cálculos de BPM e as linhas cheias representam as previsões teóricas de ZPM.

altura da barreira.

Obviamente, os processos de fusão e de espalhamento elástico poderiam ser analisados através de cálculos de CC. No entanto, como comentado no Capítulo 4, quando um número grande de estados contribui significativamente para estes processos, os cálculos de CC podem ser afetados por problemas numéricos associados à resolução das equações de canais acoplados. O modelo de ZPM, por outro lado, já inclui o efeito de um número enorme de estados inelásticos.

Em muitos trabalhos, os dados de espalhamento elástico são descritos usando alguns poucos parâmetros livres nos cálculos de modelo óptico. No presente capítulo, assumimos o potencial de São Paulo como a interação nuclear bare e uma importante característica desta interação é a ausência de parâmetros ajustáveis. Logo, nossos resultados para seções de choque teóricas de fusão e espalhamento elástico representam previsões, ao invés de ajuste de dados. Como demonstramos nas Figuras 6.2, 6.6 e 6.7, estas previsões estão razoavelmente de acordo com os dados para os sistemas ${ }^{58} \mathrm{Ni}+$ ${ }^{64} \mathrm{Ni},{ }^{16} \mathrm{O}+{ }^{58} \mathrm{Ni},{ }^{208} \mathrm{~Pb}$. 

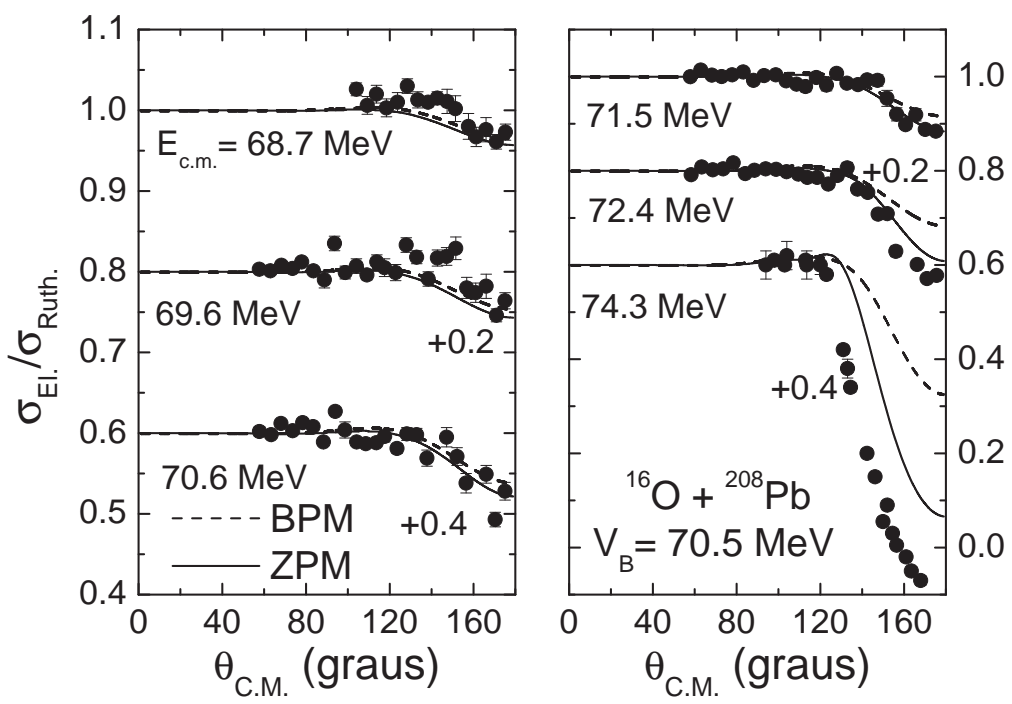

(a) ${ }^{16} \mathrm{O}+{ }^{208} \mathrm{~Pb}$.
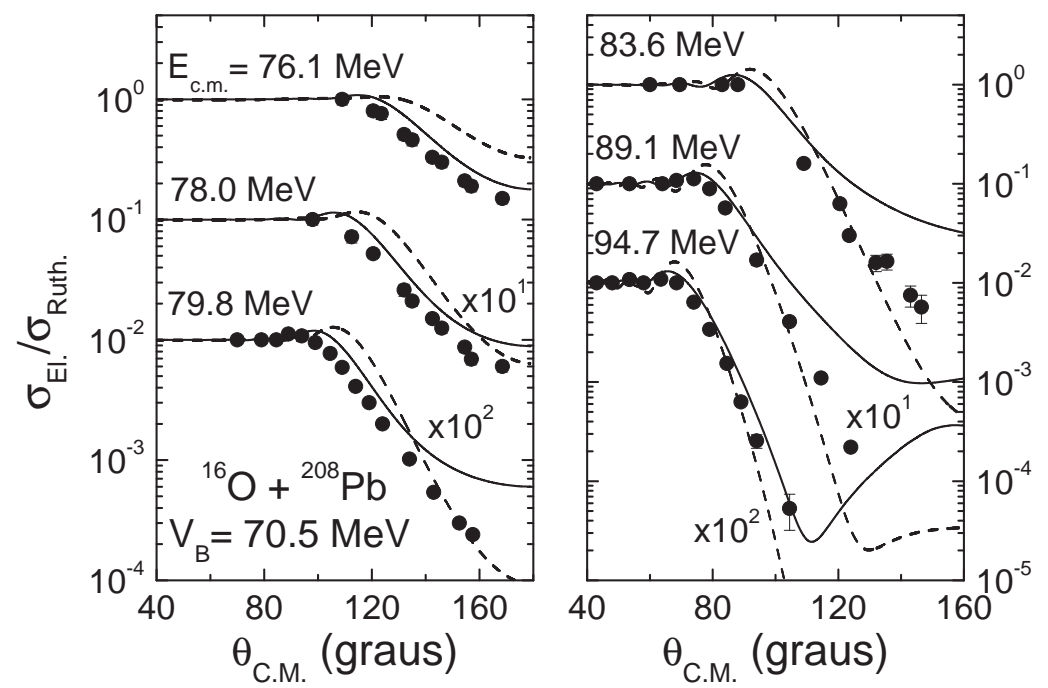

(b) ${ }^{16} \mathrm{O}+{ }^{208} \mathrm{~Pb}$.

Figura 6.7: Distribuições angulares de espalhamento elástico para o sistema ${ }^{16} \mathrm{O}+{ }^{208} \mathrm{~Pb}$ em diversas energias. As linhas tracejadas correspondem aos cálculos de BPM e as linhas cheias representam as previsões teóricas de ZPM. 


\section{Capítulo 7}

\section{Fusão de Núcleos Ímpares}

No Capítulo 4 propusemos um modelo [36], que chamamos de Movimento de Ponto Zero (ZPM), onde consideramos o efeito dos acoplamentos aos conjuntos completos de estados inelásticos conectados às bandas vibracionais de quadrupolo $\left(2^{+}\right)$e octupolo $\left(3^{-}\right)$dos núcleos par-par. No Capítulo 5 analisamos dados de fusão para 64 diferentes sistemas de íons pesados [36] sem o auxílio de qualquer parâmetros ajustável. Em energias extremamente abaixo da barreira (em torno de $20 \mathrm{MeV}$ abaixo da barreira), o modelo de penetração de barreira unidimensional (BPM) subestima os dados por aproximadamente onze ordens de magnitude, enquanto os resultados do modelo de ZPM concordam com os dados dentro de, no máximo, apenas duas ordens de grandeza para a maioria dos sistemas. Este é um importante resultado devido à falta de parâmetros ajustáveis.

No Capítulo 5 consideramos apenas sistemas envolvendo núcleos par-par pois o modelo de ZPM é baseado em características (parâmetros de deformação e energias de excitação) dos estados excitados $2^{+}$e $3^{-}$dos núcleos. No presente capítulo, estendemos os cálculos de ZPM para a fusão de sistemas envolvendo núcleos com um número ímpar de prótons e/ou nêutrons (referiremo-nos a estes núcleos, deste ponto adiante, simplesmente como núcleos ímpares). Para atingir este objetivo, apresentamos na Seção 7.1 uma sistemática das energias de excitação dos estados $2^{+}$e $3^{-}$para núcleos par-par. Usamos os resultados da sistemática apresentada na Seção 7.1 para analisar os dados de fusão na Seção 7.3. Na Seção 7.2, mostramos o desenvolvimento do modelo de ZPM para a fusão envolvendo núcleos ímpares. 


\subsection{Sistemática de Energias de Excitação}

Na Seção 3.2, com o objetivo de obter os parâmetros de deformação, usamos a sistemática para as probabilidades de transição de quadrupolo $B(E 2)$ e de octupolo $B(E 3)$ [38] para núcleos par-par das Refs. [51, 65]. Definimos os correspondentes comprimentos de deformação por $\delta_{\lambda}=\beta_{\lambda} R_{c}$, onde $R_{c}$ é dado pela Eq. (3.5) (página 27), e assumimos sua conexão com as probabilidades de transmissão como expresso pela Eq. (3.7), na página 28. Propusemos que os comprimentos de deformação podem ser aproximadamente descritos usando as funções:

$$
\delta_{\lambda}=D_{\lambda}(Z)+D_{\lambda}(N)
$$

onde $Z$ e $N$ são, respectivamente, os números de prótons e nêutrons do núcleo, $D_{3}(X)=\frac{\alpha}{\sqrt{X}}$ com $\alpha=3.2 \mathrm{fm}$, e a função $D_{2}(X)$ foi dada na Tabela 3.1 (página 31), como pode ser visto na Seção 3.2. A Eq. (7.1) descreve o conjunto completo de comprimentos de deformação extraídos experimentalmente com uma dispersão (desvio padrão) de $0.2 \mathrm{fm}$ (em torno de $15 \%$ de precisão). Esta precisão é apenas ligeiramente maior que a incerteza experimental média.

De forma a obter uma sistemática para as energias de excitação, adotamos um procedimento semelhante ao que foi assumido na Seção 3.2 para os parâmetros de deformação [38]. A Figura 7.1 mostra as energias de excitação dos estados excitados $2^{+}$e $3^{-}$para núcleos par-par, obtidos nas Refs. [51, 65], como uma função do número de prótons ou de nêutrons dos núcleos. Uma inspeção da Figura 7.1 mostra uma similaridade bastante interessante entre o comportamento dos valores de $E_{\lambda}^{*}$ como uma função de $Z$ e $N$. Com o intuito de enfatizar este comportamento, calculamos valores médios sobre isótopos e também sobre isótonos (Figura 7.2). Baseado nestas observações, muito semelhantes àquelas obtidas para os parâmetros de deformação (Seção 3.2), propomos que as energias de excitação, $E_{2}^{*}$ e $E_{3}^{*}$, podem se aproximadamente descritas usando as seguintes funções:

$$
E_{\lambda}^{*}=E_{\lambda}(Z)+E_{\lambda}(N)
$$

A partir do ajuste dos valores experimentais de $E_{\lambda}^{*}$ obtivemos tais funções, que são ilustradas na Figura 7.3 e apresentadas nas Tabelas 7.1 e 7.2. A Eq. (7.2), com as funções das Tabelas 7.1 e 7.2, 
descrevem o conjunto completo de energias de excitação com uma dispersão (desvio padrão) de 0.30 $\mathrm{MeV}$. Este valor é muito maior que as incertezas experimentais, mas é razoavelmente preciso para o objetivo de descrever o processo de fusão para núcleos ímpares através do procedimento apresentado na Seção 7.2.

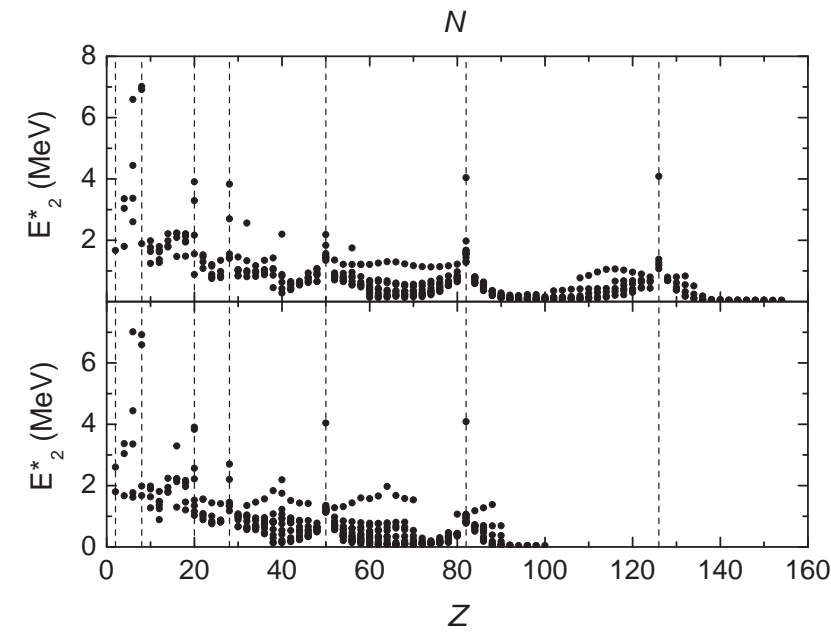

(a) Energias de excitação do estado $2^{+}$.

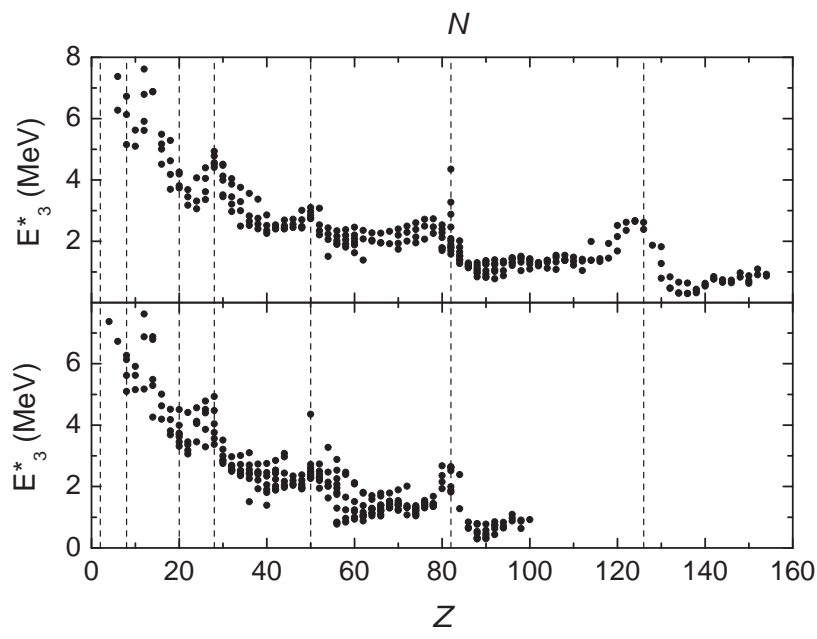

(b) Energias de excitação do estado $3^{-}$.

Figura 7.1: Valores experimentais das energias de excitação dos estados $2^{+}$e $3^{-}$como uma função do número de prótons (embaixo) ou nêutrons (em cima) dos núcleos. As linhas pontilhadas representam os números mágicos. 


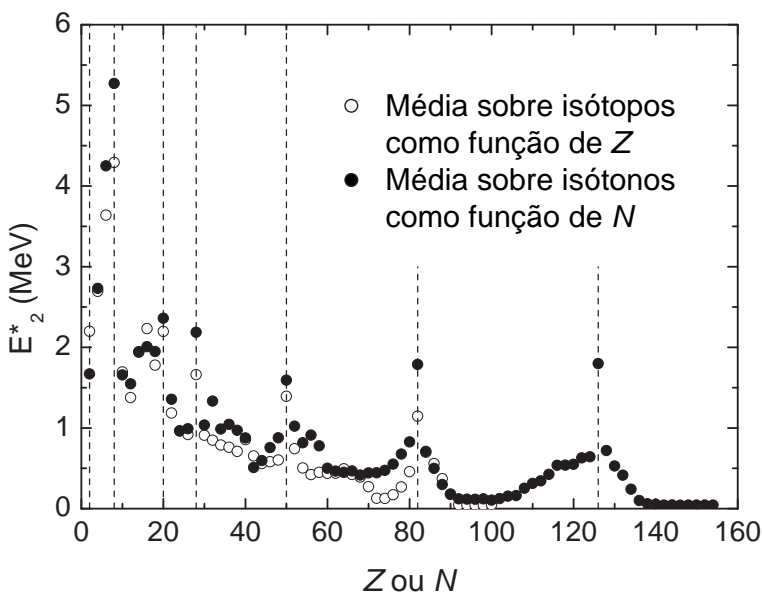

(a) Médias das energias de excitação do estado $2^{+}$.

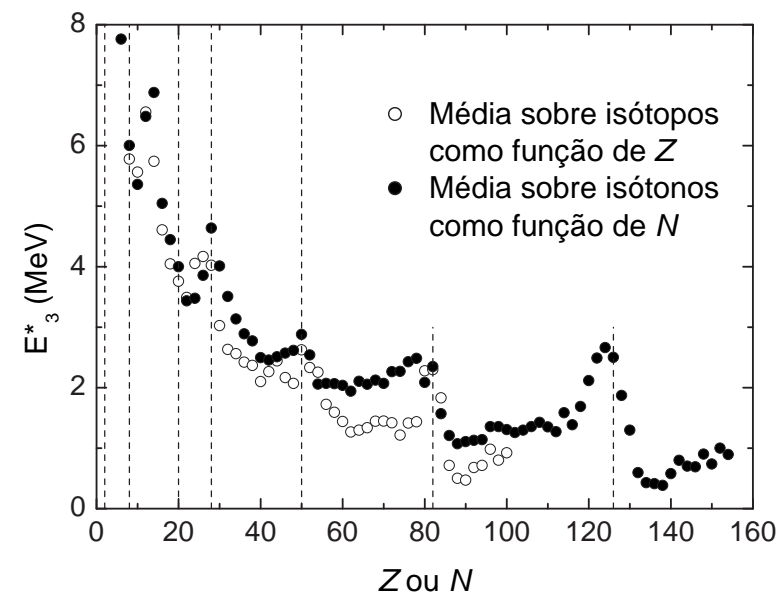

(b) Médias das energias de excitação do estado $3^{-}$.

Figura 7.2: Valores médios para isótopos (ou isótonos) das energias de excitação dos estados $2^{+}$e $3^{-}$ como uma função do número de prótons (ou nêutrons) dos núcleos.

Tabela 7.1: Valores da função $E_{2}(X)$.

\begin{tabular}{cccccccc}
\hline \hline$X$ & $E_{2}(\mathrm{MeV})$ & $X$ & $E_{2}(\mathrm{MeV})$ & $X$ & $E_{2}(\mathrm{MeV})$ & $X$ & $E_{2}(\mathrm{MeV})$ \\
\hline 2 & 0.52 & 42 & 0.16 & 82 & 1.02 & 122 & 0.13 \\
4 & 1.28 & 44 & 0.19 & 84 & 0.57 & 124 & 0.07 \\
6 & 2.09 & 46 & 0.33 & 86 & 0.36 & 126 & 0.91 \\
8 & 3.46 & 48 & 0.46 & 88 & 0.20 & 128 & 0.33 \\
10 & 0.75 & 50 & 1.07 & 90 & 0.05 & 130 & 0.11 \\
12 & 0.50 & 52 & 0.53 & 92 & 0.04 & 132 & -0.02 \\
14 & 1.11 & 54 & 0.29 & 94 & 0.02 & 134 & -0.08 \\
16 & 1.12 & 56 & 0.20 & 96 & 0.00 & 136 & -0.04 \\
18 & 0.98 & 58 & 0.18 & 98 & -0.01 & 138 & 0.01 \\
20 & 1.20 & 60 & 0.15 & 100 & -0.02 & 140 & 0.04 \\
22 & 0.53 & 62 & 0.11 & 102 & -0.03 & 142 & 0.04 \\
24 & 0.37 & 64 & 0.09 & 104 & -0.04 & 144 & 0.04 \\
26 & 0.42 & 66 & 0.09 & 106 & -0.03 & 146 & 0.04 \\
28 & 1.00 & 68 & 0.10 & 108 & -0.04 & 148 & 0.04 \\
30 & 0.50 & 70 & 0.10 & 110 & -0.03 & 150 & 0.05 \\
32 & 0.45 & 72 & 0.12 & 112 & -0.01 & 152 & 0.04 \\
34 & 0.40 & 74 & 0.14 & 114 & 0.02 & 154 & 0.06 \\
36 & 0.43 & 76 & 0.17 & 116 & 0.04 & & \\
38 & 0.46 & 78 & 0.30 & 118 & 0.05 & & \\
40 & 0.38 & 80 & 0.44 & 120 & 0.11 & & \\
\hline \hline
\end{tabular}


Tabela 7.2: Valores da função $E_{3}(X)$.

\begin{tabular}{cccccccc}
\hline \hline$X$ & $E_{3}(\mathrm{MeV})$ & $X$ & $E_{3}(\mathrm{MeV})$ & $X$ & $E_{3}(\mathrm{MeV})$ & $X$ & $E_{3}(\mathrm{MeV})$ \\
\hline 2 & 23.7 & 42 & 1.11 & 82 & 1.31 & 122 & 1.26 \\
4 & 3.30 & 44 & 1.24 & 84 & 0.77 & 124 & 1.46 \\
6 & 4.07 & 46 & 1.41 & 86 & 0.53 & 126 & 1.47 \\
8 & 2.66 & 48 & 1.45 & 88 & 0.28 & 128 & 0.56 \\
10 & 2.49 & 50 & 1.73 & 90 & 0.31 & 130 & 0.51 \\
12 & 3.45 & 52 & 1.42 & 92 & 0.39 & 132 & 0.19 \\
14 & 3.34 & 54 & 1.21 & 94 & 0.43 & 134 & 0.04 \\
16 & 2.43 & 56 & 0.92 & 96 & 0.57 & 136 & 0.01 \\
18 & 2.08 & 58 & 0.80 & 98 & 0.47 & 138 & 0.05 \\
20 & 1.76 & 60 & 0.73 & 100 & 0.48 & 140 & 0.26 \\
22 & 1.60 & 62 & 0.63 & 102 & 0.37 & 142 & 0.46 \\
24 & 1.99 & 64 & 0.60 & 104 & 0.51 & 144 & 0.29 \\
26 & 2.20 & 66 & 0.63 & 106 & 0.53 & 146 & 0.29 \\
28 & 2.57 & 68 & 0.93 & 108 & 0.58 & 148 & 0.40 \\
30 & 1.85 & 70 & 0.85 & 110 & 0.47 & 150 & 0.28 \\
32 & 1.47 & 72 & 0.79 & 112 & 0.47 & 152 & 0.51 \\
34 & 1.31 & 74 & 0.79 & 114 & 0.50 & 154 & 0.40 \\
36 & 1.20 & 76 & 0.94 & 116 & 0.46 & & \\
38 & 1.02 & 78 & 0.88 & 118 & 0.68 & & \\
40 & 1.01 & 80 & 1.15 & 120 & 1.00 & & \\
\hline \hline
\end{tabular}

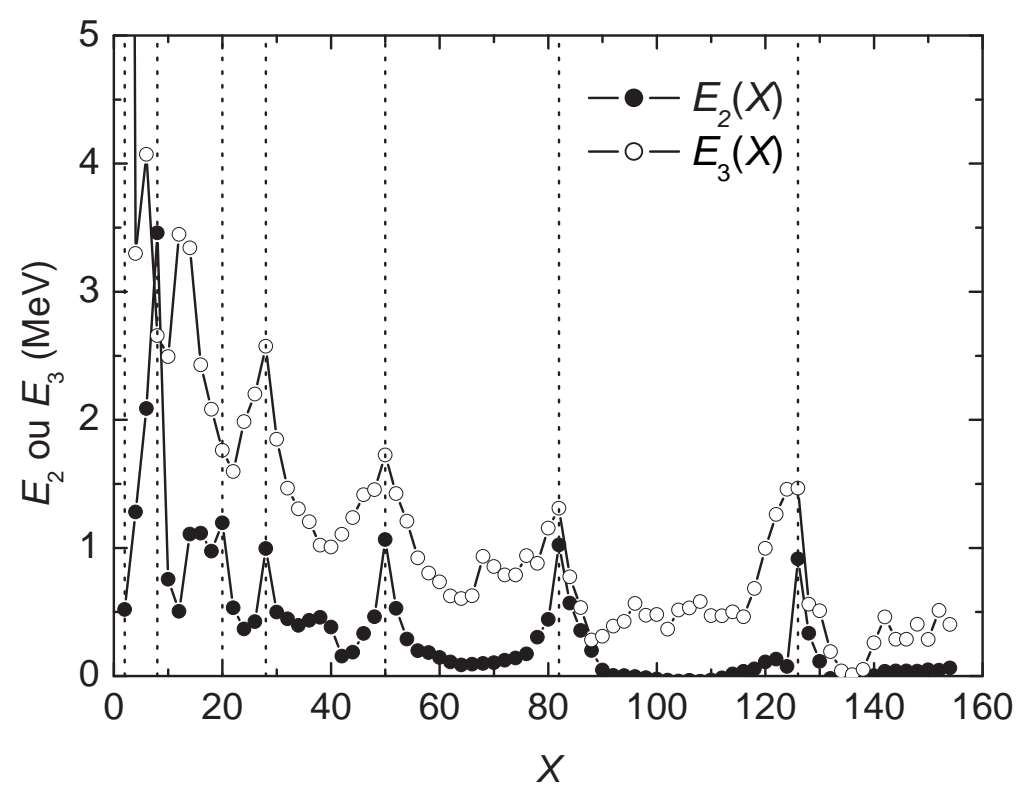

Figura 7.3: As funções $E_{2}$ e $E_{3}$ que descrevem aproximadamente o comportamento das energias de excitação. As linhas pontilhadas representam os números mágicos. 


\subsection{Modelo de ZPM para Núcleos Ímpares}

Nos Capítulos 4 e 5, aplicamos o modelo de ZPM à análise de dados de fusão de 64 diferentes sistemas envolvendo apenas núcleos par-par [36]. Os valores de $\beta$ dos modos de vibração foram conectados, através da Eq. (3.7) da página 28, com as correspondentes amplitudes de transição experimentais de quadrupolo $(B E 2)$ e de octupolo ( $B E 3)$ obtidas nas Refs. [51, 65]. As energias de excitação dos estados $2^{+}$e $3^{-}$foram também obtidas nas mesmas referências.

O objetivo do presente capítulo é estender os cálculos de ZPM a sistemas envolvendo núcleos ímpares. Isto foi feito baseado na idéia de que núcleos ímpares deveriam ter vibrações coletivas semelhantes aos núcleos par-par. As análises foram efetuadas sem qualquer parâmetro ajustável, assumindo os valores médios de raio e difusividade para a densidade nuclear. Para estimar os valores de $\beta$ e $E^{*}$, usamos as sistemáticas apresentadas nas Seções 3.2 e 7.1. Assumimos as Eqs. (7.1) e (7.2) e, para valores ímpares de $X$, interpolamos as funções $D_{\lambda}$ e $E_{\lambda}$ entre os dois valores pares vizinhos de $X$. Obviamente, este procedimento foi aplicado apenas para núcleos ímpares, pois, para núcleos par-par, assumimos os correspondentes valores experimentais de $B E 2, B E 3, E_{2}^{*}$ e $E_{3}^{*}$ das Refs. [51, 65]. O procedimento de interpolação dos parâmetros não é justificado a priori, pois partículas desemparelhadas poderiam afetar de uma forma não linear as propriedades coletivas dos núcleos. No entanto, assumimos esta hipótese de maneira a testá-la na descrição do processo de fusão.

\subsection{Comparação com Dados e Discussão dos Resultados}

Na Tabela 7.3, apresentamos os núcleos envolvidos nos 48 sistemas aqui estudados, com os correspondentes valores de $\beta$ e $E^{*}$ assumidos para os modos de quadrupolo e de octupolo. As Figuras 7.4, 7.5 e 7.8 apresentam os dados de fusão (obtidos das referências fornecidas nas legendas) e os correspondentes resultados de cálculos de BPM não-deformado (linhas tracejadas) e de ZPM (linhas cheias). A escala de energia é representada relativa a altura da barreira da onda s não-deformada $\left(V_{B}\right)$. As figuras possuem a mesma escala de energia e de seção de choque. Este procedimento torna mais simples a comparação entre os resultados de diferentes sistemas.

A Figura 7.4(a) apresenta dados de sistemas envolvendo ${ }^{10,11} \mathrm{~B}$ como projéteis. Para o sistema leve

${ }^{11} \mathrm{~B}+{ }^{14} \mathrm{~N}$, os resultados de ZPM e BPM são quase indistinguíveis e concordam de forma bastante 
Tabela 7.3: A tabela apresenta os valores dos parâmetros de deformação de quadrupolo $\left(\beta_{2}\right)$ e de octupolo $\left(\beta_{3}\right)$, e também das correspondentes energias de excitação para os núcleos estudados neste capítulo.

\begin{tabular}{|c|c|c|c|c|}
\hline núcleo & $\overline{\beta_{2}}$ & 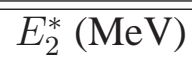 & $\overline{\beta_{3}}$ & $E_{3}^{*}(\mathrm{MeV})$ \\
\hline${ }^{6} \mathrm{Li}$ & 3.11 & 1.80 & 2.34 & 27.0 \\
\hline${ }^{7} \mathrm{Li}$ & 3.01 & 2.18 & 2.18 & 16.8 \\
\hline${ }^{9} \mathrm{Be}$ & 2.15 & 2.97 & 1.65 & 6.98 \\
\hline${ }^{10} \mathrm{~B}$ & 1.60 & 3.37 & 1.40 & 7.37 \\
\hline${ }^{11} \mathrm{~B}$ & 1.28 & 3.77 & 1.34 & 7.76 \\
\hline${ }^{14} \mathrm{~N}$ & 0.56 & 5.55 & 1.01 & 6.73 \\
\hline${ }^{15} \mathrm{~N}$ & 0.43 & 6.23 & 0.97 & 6.02 \\
\hline${ }^{16} \mathrm{O}$ & 0.50 & 6.92 & 1.20 & 6.13 \\
\hline${ }^{17} \mathrm{O}$ & 0.48 & 5.57 & 0.86 & 5.23 \\
\hline${ }^{18} \mathrm{O}$ & 0.54 & 1.98 & 1.10 & 5.10 \\
\hline${ }^{19} \mathrm{~F}$ & 0.83 & 2.86 & 0.77 & 5.07 \\
\hline${ }^{27} \mathrm{Al}$ & 0.46 & 1.91 & 0.55 & 6.74 \\
\hline${ }^{32} \mathrm{~S}$ & 0.38 & 2.23 & 0.70 & 5.01 \\
\hline${ }^{36} \mathrm{~S}$ & 0.32 & 3.29 & 0.56 & 4.19 \\
\hline${ }^{35} \mathrm{Cl}$ & 0.32 & 2.02 & 0.43 & 4.34 \\
\hline${ }^{37} \mathrm{Cl}$ & 0.24 & 2.24 & 0.42 & 4.02 \\
\hline${ }^{45} \mathrm{Sc}$ & 0.32 & 1.23 & 0.35 & 3.66 \\
\hline${ }^{46} \mathrm{Ti}$ & 0.37 & 0.89 & 0.18 & 3.06 \\
\hline${ }^{50} \mathrm{Ti}$ & 0.21 & 1.55 & 0.19 & 4.41 \\
\hline${ }^{51} \mathrm{~V}$ & 0.25 & 1.45 & 0.31 & 4.37 \\
\hline${ }^{59} \mathrm{Co}$ & 0.25 & 1.16 & 0.27 & 3.85 \\
\hline${ }^{58} \mathrm{Ni}$ & 0.21 & 1.45 & 0.24 & 4.48 \\
\hline${ }^{60} \mathrm{Ni}$ & 0.24 & 1.33 & 0.26 & 4.04 \\
\hline${ }^{62} \mathrm{Ni}$ & 0.23 & 1.17 & 0.25 & 2.75 \\
\hline${ }^{64} \mathrm{Ni}$ & 0.22 & 1.35 & 0.26 & 3.56 \\
\hline${ }^{65} \mathrm{Cu}$ & 0.26 & 1.18 & 0.25 & 3.42 \\
\hline${ }^{70} \mathrm{Ge}$ & 0.26 & 1.04 & 0.33 & 2.56 \\
\hline${ }^{73} \mathrm{Ge}$ & 0.29 & 0.72 & 0.23 & 2.53 \\
\hline${ }^{74} \mathrm{Ge}$ & 0.33 & 0.60 & 0.19 & 2.54 \\
\hline${ }^{76} \mathrm{Ge}$ & 0.32 & 0.56 & 0.19 & 2.69 \\
\hline${ }^{89} \mathrm{Y}$ & 0.14 & 1.49 & 0.19 & 2.74 \\
\hline${ }^{92} \mathrm{Zr}$ & 0.12 & 0.93 & 0.21 & 2.34 \\
\hline${ }^{93} \mathrm{Nb}$ & 0.20 & 0.80 & 0.18 & 2.48 \\
\hline${ }^{101} \mathrm{Ru}$ & 0.28 & 0.37 & 0.17 & 2.10 \\
\hline${ }^{103} \mathrm{Rh}$ & 0.26 & 0.44 & 0.17 & 2.13 \\
\hline${ }^{105} \mathrm{Pd}$ & 0.25 & 0.50 & 0.17 & 2.18 \\
\hline${ }^{112} \mathrm{Sn}$ & 0.14 & 1.26 & 0.15 & 2.36 \\
\hline${ }^{144} \mathrm{Sm}$ & 0.10 & 1.66 & 0.17 & 1.81 \\
\hline${ }^{159} \mathrm{~Tb}$ & 0.37 & 0.09 & 0.12 & 1.04 \\
\hline${ }^{165} \mathrm{Ho}$ & 0.39 & 0.08 & 0.12 & 1.25 \\
\hline${ }^{208} \mathrm{~Pb}$ & 0.06 & 4.08 & 0.13 & 2.61 \\
\hline${ }^{209} \mathrm{Bi}$ & 0.014 & 1.71 & 0.10 & 2.51 \\
\hline${ }^{232} \mathrm{Th}$ & 0.30 & 0.05 & 0.10 & 0.77 \\
\hline
\end{tabular}


precisa com os dados em toda a região de energia. Para os sistemas mais pesados, enhancements significativos dos dados em relação aos cálculos de BPM podem ser observados, enquanto a concordância entre os dados e os resultados de ZPM é bastante razoável. As Figuras 7.4(b), 7.4(c) e 7.4(d) apresentam sistemas envolvendo isótopos de nitrogênio, flúor, oxigênio e alumínio como projéteis. Uma concordância bastante razoável entre os dados e os resultados de ZPM é observada para todos os sistemas. As Figuras 7.5(a), 7.5(b), 7.5(c), 7.5(d) e 7.5(e) mostram sistemas envolvendo isótopos de enxofre e cloro como projéteis e, novamente, previsões bastante razoáveis do modelo de ZPM são obtidas, com algumas discordâncias observadas para ${ }^{36} \mathrm{~S}$ (ver Figura 7.5(b)). A Figura 7.5(f) apresenta os sistemas mais pesados estudados neste capítulo, e apenas para o sistema ${ }^{60} \mathrm{Ni}+$ ${ }^{89}$ Y o modelo de ZPM não é muito preciso na descrição dos dados abaixo da barreira.

Na Figura 7.6, apresentamos um resumo dos resultados mostrados nas Figuras 7.4 e 7.5, calculando a razão entre os dados de fusão e as correspondentes seções de choque de BPM ou ZPM. Para comparação, a figura também contém os resultados para sistemas envolvendo apenas núcleos par-par, discutidos em detalhes no Capítulo 5 e resumidos na Figura 5.8 da página 67. Na Figura 7.6, na parte relativa aos núcleos par-par, também foram incluídos os sistemas ${ }^{40} \mathrm{Ca},{ }^{40} \mathrm{Ar}+{ }^{116} \mathrm{Sn}$, apresentados na Figura 5.9 (página 69), que não haviam sido incluídos na Figura 5.8. Em energias extremamente abaixo da barreira, os dados são subestimados pelos cálculos de BPM por onze ordens de magnitude. Por outro lado, quase todas as seções de choque de ZPM concordam com os dados dentro de apenas duas ordens de grandeza. Levando em consideração a ausência de parâmetros ajustáveis, a concordância, de uma forma geral, entre dados e seções de choque de ZPM pode ser considerada bastante boa. Devemos observar na Figura 7.6 que nenhuma diferença significativa entre os resultados para sistemas envolvendo núcleos ímpares e apenas par-par é observada. Para enfatizar este ponto, calculamos valores médios para a ordem de magnitude dos desvios entre os dados de fusão e as seções de choque de ZPM. Dividimos os dados apresentados na Figura 7.6 (painéis da direita) em passos consecutivos de $2 \mathrm{MeV}$. Para cada passo calculamos o valor médio de $\ln \left(\sigma_{F} / \sigma_{Z P M}\right)$, e definimos valores médios para a ordem de magnitude através da seguinte expressão $\left\langle\sigma_{F} / \sigma_{Z P M}\right\rangle=e^{\left\langle\ln \left(\sigma_{F} / \sigma_{Z P M}\right)\right\rangle}$. Os resultados correspondentes são apresentados na Figura 7.7(a). Os valores médios para núcleos ímpares e par-par são bastante semelhantes para $E_{c . m .}-V_{B} \geq-13 \mathrm{MeV}$. Para $E_{c . m \text {. }}-V_{B}<-15 \mathrm{MeV}$, grandes diferenças entre os dois conjuntos de sistemas podem ser observadas. No entanto, consideramos que 


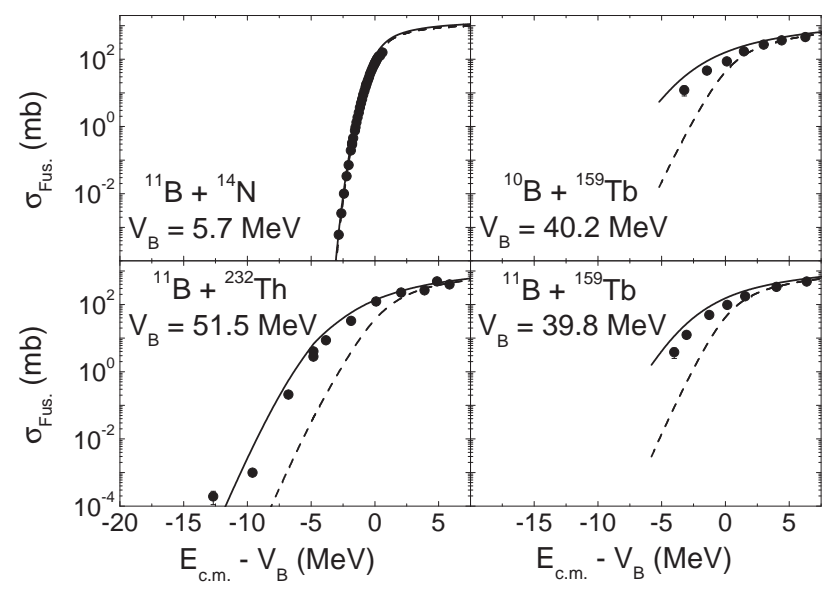

(a) Os dados são das Refs. [104-106].

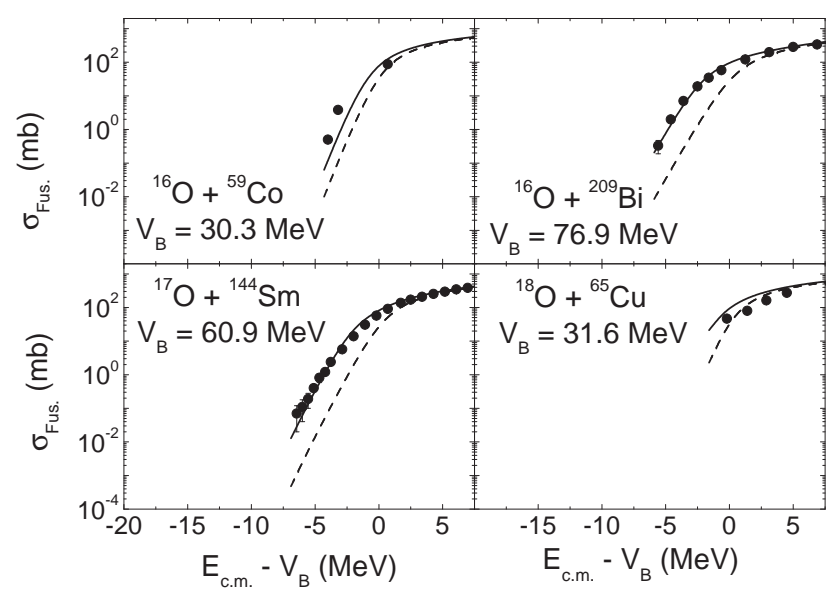

(c) Os dados são das Refs. [77, 79, 107, 109].

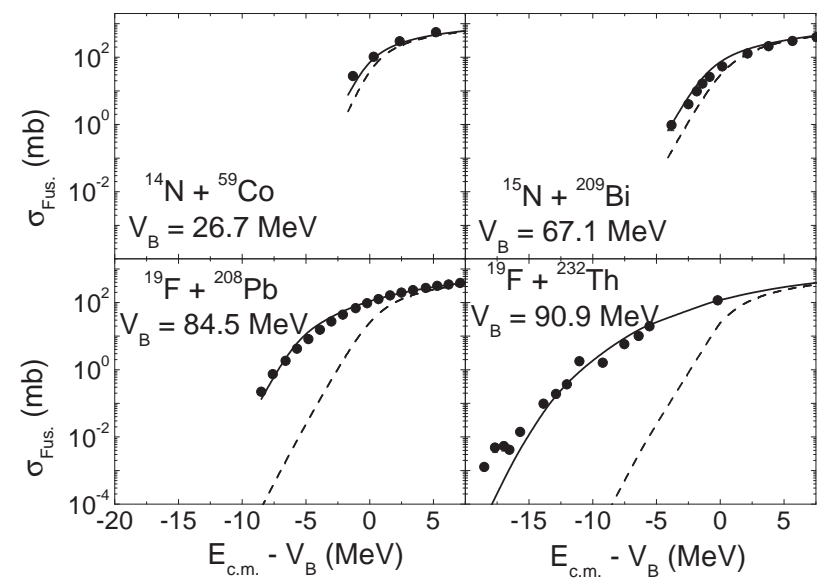

(b) Os dados são das Refs. [77, 105, 107, 108].

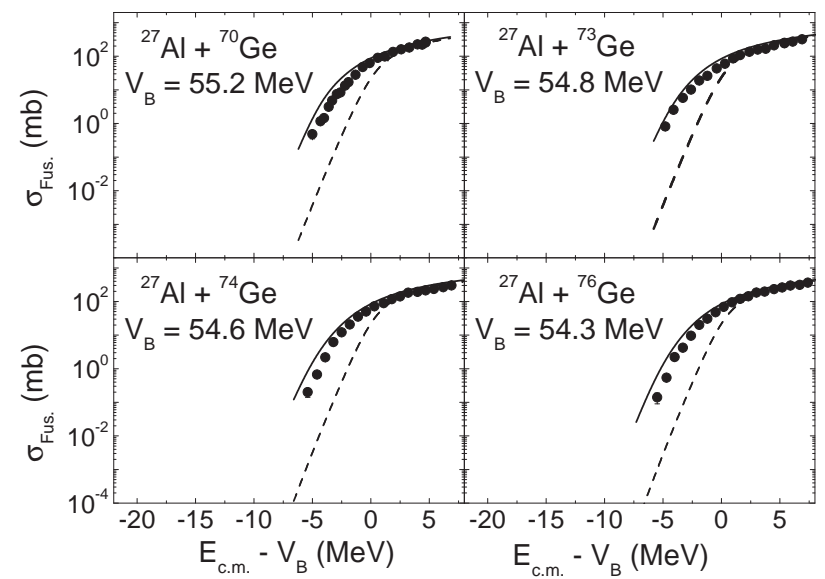

(d) Os dados são da Ref. [110].

Figura 7.4: Dados de fusão e correspondentes seções de choque teóricas de BPM não-deformada (linhas tracejadas) e de ZPM (linhas cheias) para os sistemas indicados nas figuras. As alturas da barreira da onda s do potencial não-deformado são apresentadas nas figuras. 


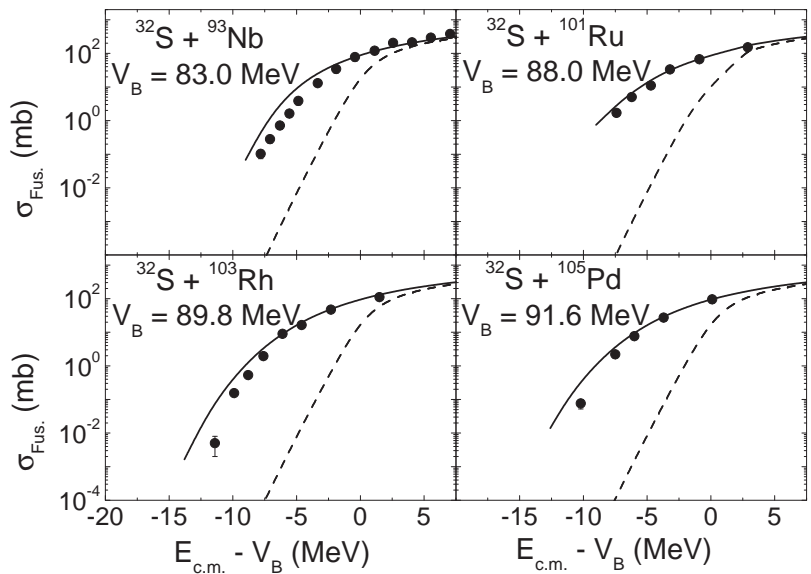

(a) Os dados são das Refs. [86, 111].

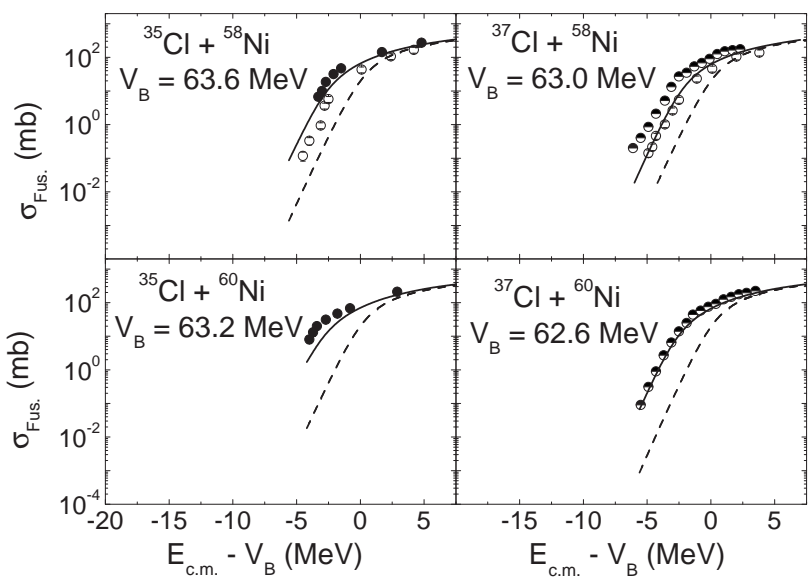

(c) Os dados são das Refs. [112-114].

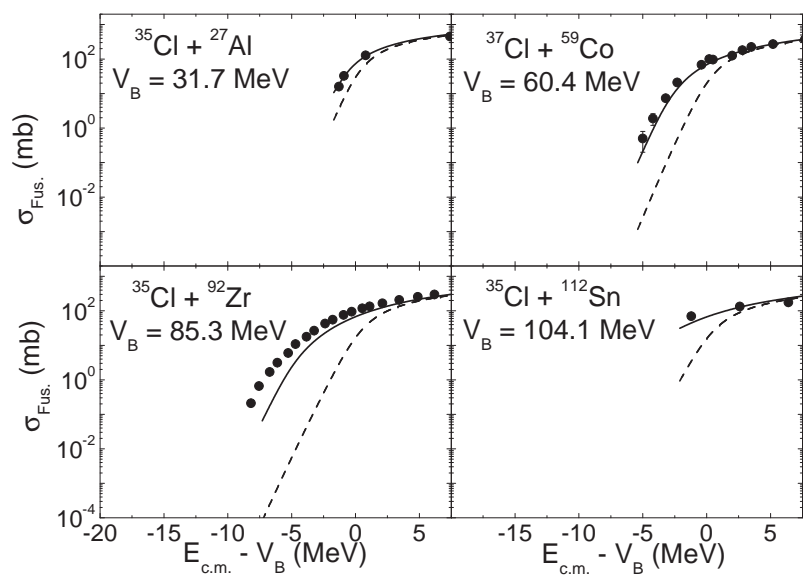

(e) Os dados são das Refs. [75, 84, 112, 115].

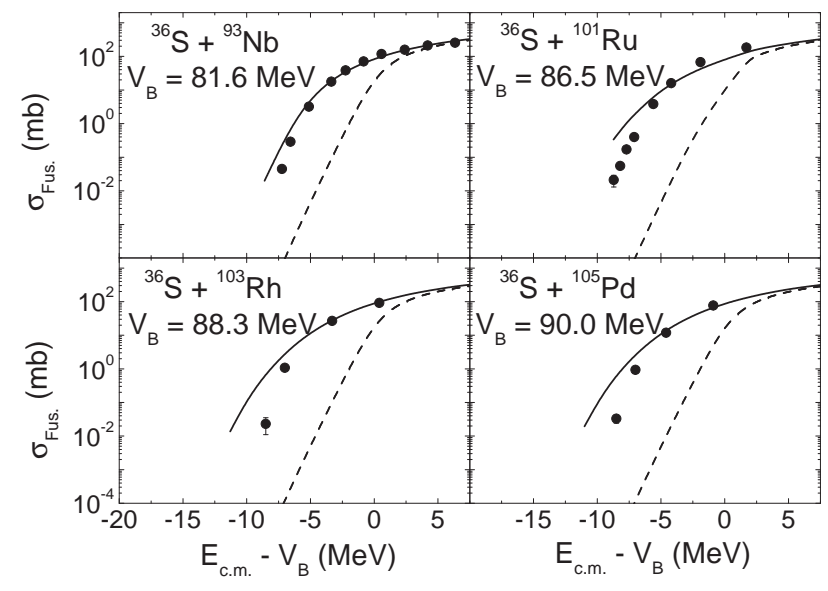

(b) Os dados são das Refs. [86, 111].

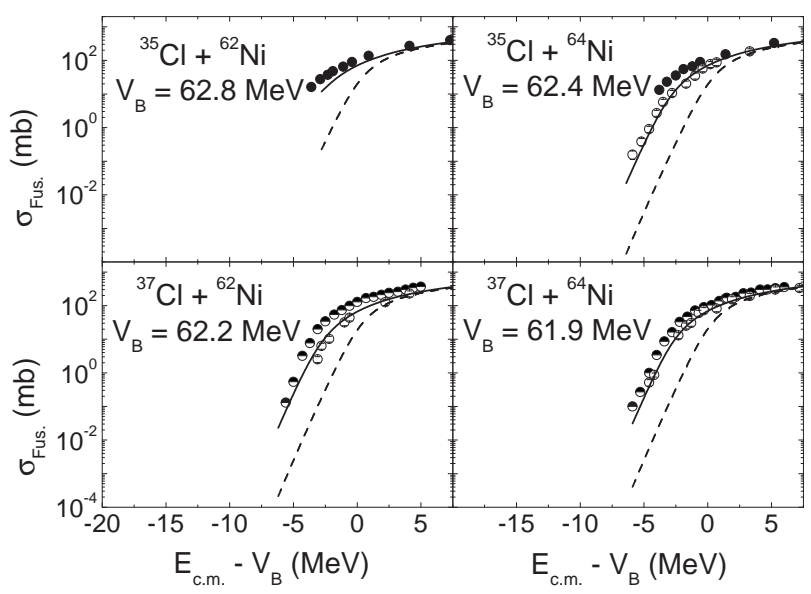

(d) Os dados são das Refs. [112-114].

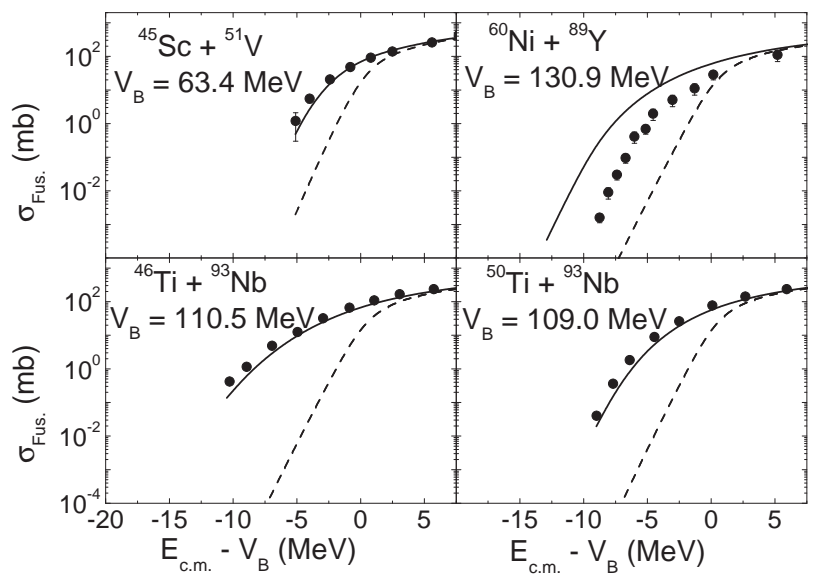

(f) Os dados são das Refs. [9, 84, 111].

Figura 7.5: Dados de fusão e correspondentes seções de choque teóricas de BPM não-deformada (linhas tracejadas) e de ZPM (linhas cheias) para os sistemas indicados nas figuras. As alturas da barreira da onda s do potencial não-deformado são apresentadas nas figuras. Em alguns casos, existem diferentes conjuntos de dados (indicados por diferentes símbolos) com seções de choque medidas que diferem por até uma ordem de magnitude. 

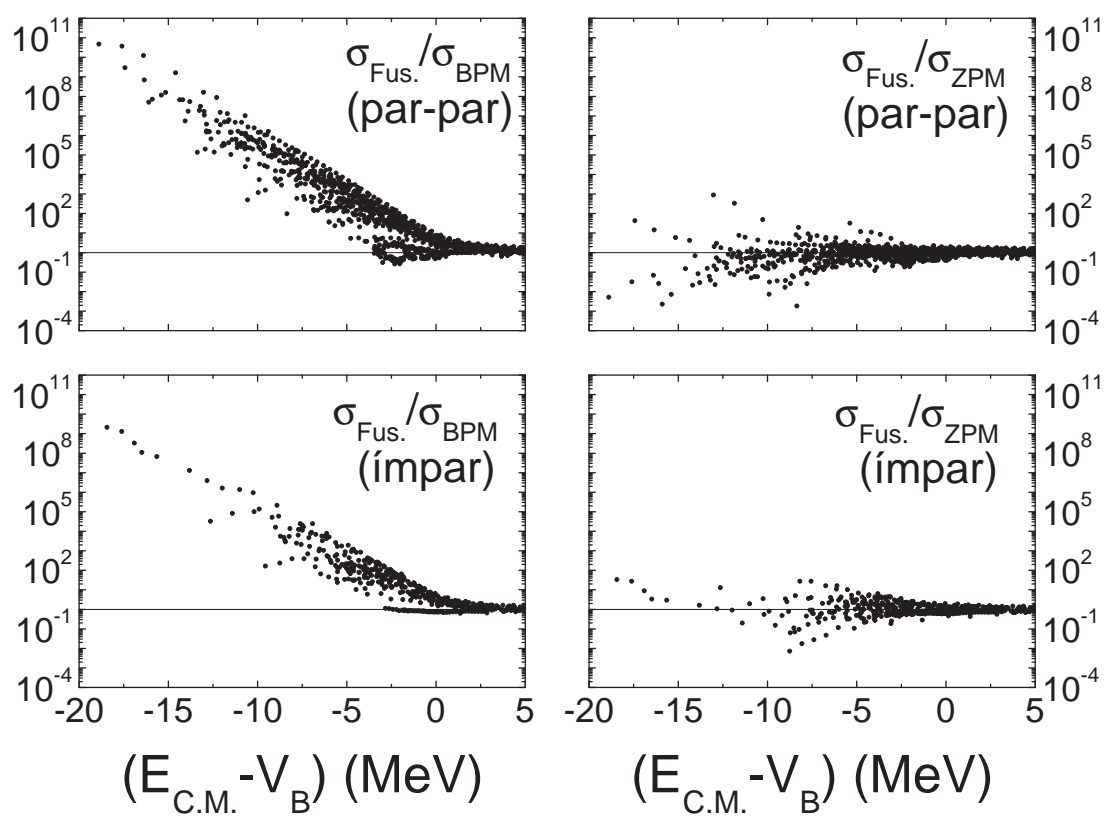

Figura 7.6: Razões entre dados de fusão e seções de choque teóricas de BPM (lado esquerdo) ou ZPM (lado direito) para sistemas envolvendo núcleos ímpares (painéis de baixo) ou apenas par-par (painéis de cima).

essas discrepâncias não são significativas pelas razões expostas a seguir.

Nenhum parâmetro livre foi assumido em nossos cálculos de ZPM, porém, as amplitudes de acoplamento (valores de $\beta$ ) para núcleos par-par foram obtidas a partir dos valores experimentais de $B E \lambda$, que possuem incertezas experimentais. Para núcleos ímpares, os valores de $\beta$ e $E^{*}$ foram obtidos por interpolação e, conseqüentemente, as "incertezas" destes parâmetros são ainda maiores que aquelas para os núcleos par-par. Além disso, assumimos os valores médios para os parâmetros de densidade mas, como já foi comentado, os parâmetros de raio e difusividade mostram pequenas variações em torno dos correspondentes valores médios ao longo da tabela periódica. Logo, já seria esperado uma dispersão dos resultados de ZPM ao redor dos dados de fusão devido a flutuações dos parâmetros de acoplamento e de densidade relativos aos valores médios assumidos em nossos cálculos. Como a região de baixa energia é mais sensível aos efeitos dos acoplamentos, resultando nos grandes enhancements observados, é justamente nesta região que obteremos maior dispersão em relação aos dados, o que implicaria também em uma maior dispersão dos valores médios. Obviamente, quando um grande número de dados é considerado, como na região $E_{c . m \text {. }}-V_{B} \geq-13 \mathrm{MeV}$, estas flu- 


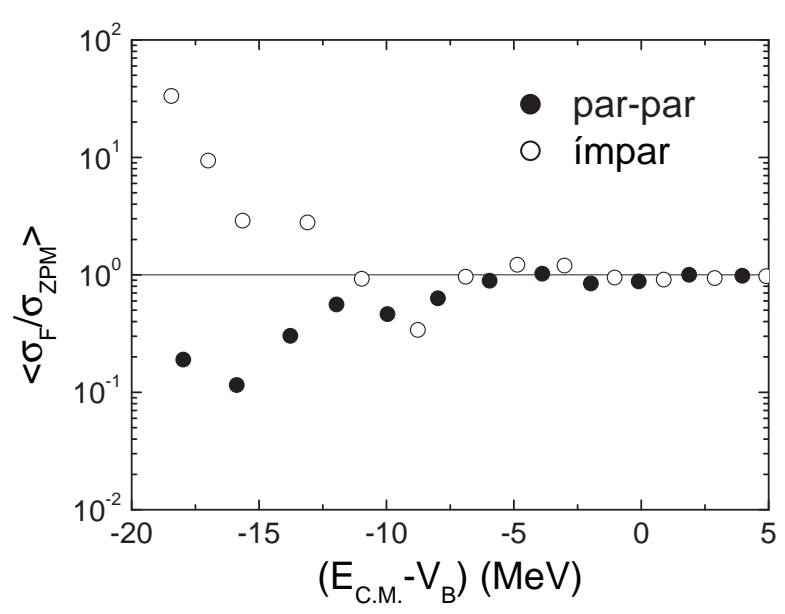

(a) Médias para sistemas envolvendo núcleos ímpares ou par-par.

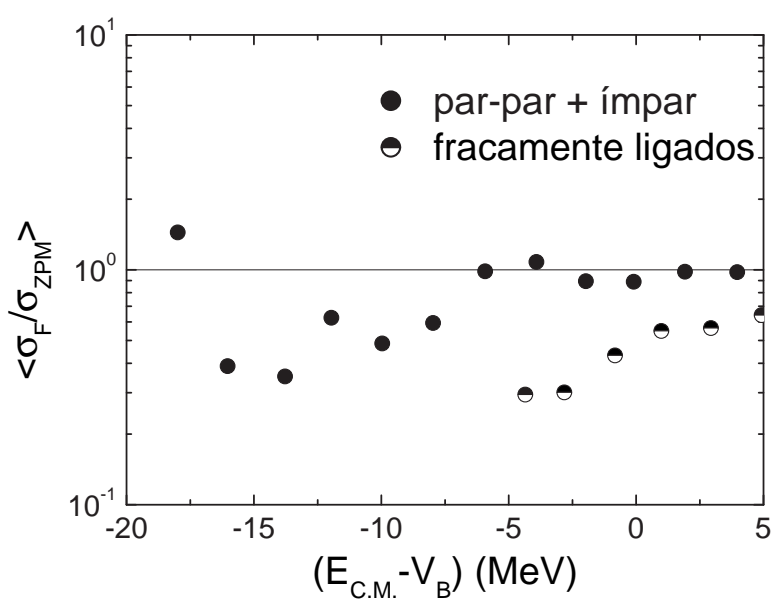

(b) Médias para sistemas envolvendo núcleos ímpares e par-par. Apresentamos também médias para os sistemas com núcleos fracamente ligados.

Figura 7.7: Valores médios da ordem de magnitude dos desvios entre dados de fusão e seções de choque de ZPM para sistemas envolvendo núcleos ímpares elou par-par e também para sistemas envolvendo os núcleos fracamente ligados ${ }^{6,7} \mathrm{Li} e^{9} \mathrm{Be}$.

tuações deveriam se cancelar parcialmente no cálculo de valores médios. No entanto, para pequenas estatísticas, a flutuação pode contaminar os valores médios de maneira mais acentuada. Esse é justamente o caso da região de energia $E_{c . m}$. $-V_{B}<-15 \mathrm{MeV}$, onde existe apenas um pequeno número de dados disponíveis (ver Figura 7.6), que correspondem somente a cinco diferentes sistemas, sendo quatro relativos a sistemas envolvendo núcleos par-par e apenas um envolvendo núcleos ímpares.

Neste sentido, a diferença observada entre valores médios de núcleos par-par e ímpares em energias muito baixas (ver Figura 7.7(a)) não é, na verdade, muito significativa. Logo, consideramos o conjunto total de núcleos par-par e ímpares e calculamos os valores médios correspondentes, que são apresentados na Figura 7.7(b). Nesta figura, podemos observar que, em média, os resultados de ZPM concordam com os dados de fusão dentro da mesma ordem de magnitude em toda a região de energia considerada neste trabalho. Apenas em energias extremamente abaixo da barreira o modelo de ZPM superestima ligeiramente as seções de choque medidas por um fator em torno de dois. Consideramos esse resultado geral como um importante sucesso do modelo de ZPM na descrição do processo de fusão entre íons pesados.

A Figura 7.8 mostra sistemas envolvendo o núcleos fracamente ligados ${ }^{6,7} \mathrm{Li} \mathrm{e}{ }^{9} \mathrm{Be}$. Aqui, uma 


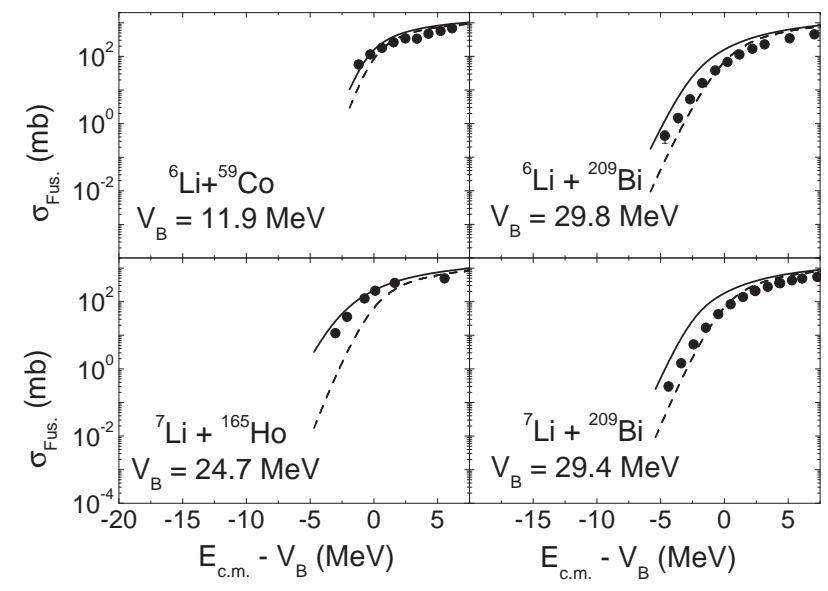

(a) Os dados são das Refs. [118-120].

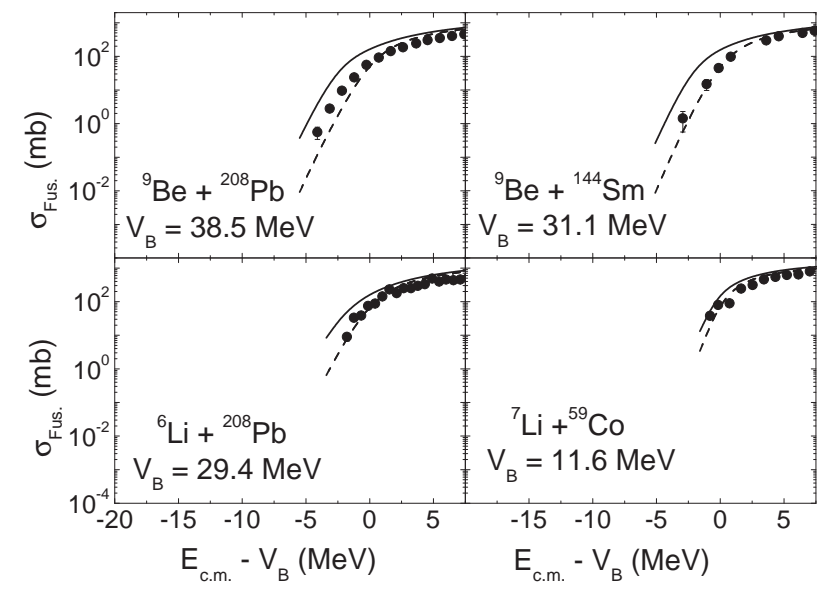

(b) Os dados são das Refs. [35, 118, 119, 121].

Figura 7.8: Dados de fusão e correspondentes seções de choque teóricas de BPM não-deformada (linhas tracejadas) e de ZPM (linhas cheias) para os sistemas indicados nas figuras. As alturas da barreira da onda s do potencial não-deformado são apresentadas nas figuras.

importante diferença em relação aos outros sistemas é observada. Claramente, existe um hindrance dos dados em relação aos resultados de ZPM mesmo em energias acima da barreira. Para ilustrar este ponto, os correspondentes valores médios são também apresentados na Figura 7.7(b). Uma possível explicação para este hindrance é a competição do processo de break-up com o de fusão, que deve ser significativo para sistemas envolvendo núcleos fracamente ligados, como já discutido em diversos trabalhos (ver e.g. [116, 117]). Enfatizamos, novamente, a importância de não se utilizar parâmetros ajustáveis nos cálculos teóricos, pois a diferença claramente estabelecida para núcleos fracamente ligados está baseada no sucesso do modelo na descrição de núcleos fortemente ligados.

O modelo de ZPM leva em consideração o efeito dos modos harmônicos de vibração no cálculo dos coeficientes de transmissão e, conseqüentemente, descreve um grande número de acoplamentos a estados inelásticos. O processo de tunelamento depende fortemente da altura da barreira, que é relacionada a variações da distância entre as superfícies dos núcleos. Logo, a fusão de íons pesados é bastante sensível a vibrações das densidades nucleares. Em nossos cálculos, assumimos o potencial de SP no contexto da sistemática das densidades nucleares. Dessa forma, nenhum parâmetro livre foi assumido em nossas análises. Descrevemos dados de fusão para 48 sistemas de íons pesados envolvendo núcleos ímpares, para os quais os parâmetros de acoplamento foram obtidos através 
da sistemática dos núcleos par-par exposta na Seção 3.2. Considerando a ausência de parâmetros ajustáveis, a concordância geral entre os dados de fusão e as previsões teóricas de ZPM pode ser considerada bastante satisfatória, dentro de apenas duas ordens de magnitude para quase todos os sistemas, incluindo a região de energia extremamente abaixo da barreira. Logo, os grandes enhancements dos dados em relação aos cálculos de BPM não-deformado, que alcançam até onze ordens de grandeza em energias muito baixa, são, em sua maioria, devidos aos efeitos dos acoplamentos a estados inelásticos. Os resultados aqui obtidos para núcleos ímpares são semelhantes àqueles anteriormente apresentados [36] para núcleos par-par no Capítulo 5. A exceção corresponde a sistemas envolvendo os núcleos fracamente ligados ${ }^{6,7} \mathrm{Li} \mathrm{e}{ }^{9} \mathrm{Be}$, onde um hindrance dos dados em relação às seções de choque de ZPM foi observado, mesmo em energias acima da barreira. Este efeito pode ser devido à competição do processo de break-up com a fusão. 


\section{Capítulo 8}

\section{Conclusão}

Nesta tese de doutoramento foram propostos dois modelos para a obtenção da seção de choque de fusão (ZPM e GFA) e um para o cálculo do potencial de polarização para o canal elástico e correspondente seção de choque de espalhamento (ZPM).

Foi usado em todos os cálculos deste trabalho, tanto no tratamento da fusão quanto no do espalhamento elástico, o Potencial de São Paulo (SP), que possui como uma das principais características a total ausência de parâmetros ajustáveis. Conseqüientemente, todos os cálculos apresentados no contexto de diferentes modelos constituem previsões teóricas ao invés de simples ajustes de dados. Apesar de o potencial de SP ser baseado em sistemáticas de densidades, o que permitiria uma certa flexibilidade nos parâmetros utilizados, este ajuste deve ser feito de forma criteriosa, de forma a lidar somente com valores de parâmetros dentro de intervalos realistas. Mostramos como a reprodução dos dados poderia ser melhorada assumindo a difusividade do potencial ou os parâmetros de deformação como sendo parâmetros livres e verificamos que, em alguns casos, teríamos que assumir valores não realistas, abdicando da coerência alcançada entre as análises de fusão e espalhamento. Em muitos casos, a ausência de parâmetros ajustáveis permite observar, claramente, características de determinados conjuntos de dados ou sistemas, como, por exemplo, no caso de núcleos fracamente ligados e também no caso dos sistemas ${ }^{90} \mathrm{Zr}+{ }^{90,96} \mathrm{Zr}$ (Figura 5.5(b), página 65), onde a contribuição de processos de evaporação de resíduos não pode ser associada à fusão total.

Em uma análise comparativa acerca dos cálculos para a fusão, os modelos GFA e ZPM se mostraram equivalentes e compatíveis com os cálculos de CC, com as vantagens de incorporarem acopla- 
mentos à toda banda vibracional e de permitirem a obtenção de cálculos acurados de seções de choque de fusão mesmo em energias extremamente abaixo da barreira. Aplicamos o modelo de ZPM ao cálculo da função de excitação de fusão para 64 sistemas de íons pesados envolvendo núcleos par-par e confrontamos os resultados obtidos com dados experimentais existentes na literatura. As previsões do modelo de ZPM mostraram-se de acordo com os dados, reproduzindo as seções de choque medidas dentro de duas ordens de magnitude, enquanto o BPM não-deformado prevê até onze ordens de grandeza de discrepância. A boa concordância obtida entre as previsões de ZPM para o processo de fusão e os dados experimentais indica que os canais mais relevantes a serem considerados são os canais inelásticos, correspondendo à maior parte do enhancement observado, e que os canais de transferência devem corresponder a efeitos de segunda ordem, dependendo da estrutura particular de cada núcleo. No entanto, como pôde ser verificado ao compararmos cálculos de CC com o modelo ZPM de dois canais, acoplamentos a estados de maior energia das bandas vibracionais são muito importantes para o processo de fusão e devem ser considerados, o que constitui uma qualidade do modelo de ZPM. Constatamos também que, ao tentarmos acoplar diversos canais em cálculos de CC, obtemos resultados inacurados e divergências numéricas em energias extremamente abaixo da barreira, advindos de integração em regiões classicamente proibidas, envolvendo diferenças de números grandes que resultam em quantidades muito pequenas. O modelo de ZPM, por outro lado, pode ser calculado até energias arbitrariamente pequenas.

Após sistemática das energias de excitação e dos parâmetros de deformação associados aos estados $2^{+}$e $3^{-}$dos núcleos par-par, ajustamos e interpolamos funções que nos permitissem estimar quais seriam os correspondentes valores no caso de núcleos ímpares. Com esta abordagem aplicamos o modelo de ZPM ao cálculo da seção de choque de fusão de diversos sistemas envolvendo núcleos ímpares (par-ímpar e/ou ímpar-ímpar) com o intuito de reproduzir os dados experimentais com seus respectivos enhancements em relação ao BPM. Os resultados obtidos mostraram-se tão bons quanto aqueles para os núcleos par-par, com exceção dos sistemas envolvendo núcleos fracamente ligados. Este resultado é provavelmente devido à competição entre o processo de fusão estudado e o processo de break-up, já conhecido como sendo importante em reações envolvendo tais núcleos.

Em uma primeira impressão, a precisão com que o modelo de ZPM descreve os dados de fusão, de duas ordens de magnitude, pode parecer insatisfatória. Entretanto, como já exaustivamente comen- 
tado, os cálculos teóricos envolveram parâmetros médios de densidades e acoplamentos, parâmetros esses que, na verdade, variam em torno de seus respectivos valores médios ao longo da tabela periódica. Então, já seriam esperadas flutuações das seções de choque teóricas relativamente aos dados experimentais, principalmente na região de baixa energia, onde os efeitos dos acoplamentos são mais importantes. Considerando médias entre muitos sistemas, tais flutuações deveriam parcialmente se anular e, de fato, obtém-se um fator dois na descrição dos dados de fusão pelo modelo de ZPM (vide Figura 7.7(b), página 94). Essa precisão de um fator dois pode ser considerada extraordinária, tendo em vista que o modelo de BPM subestima os dados em onze ordens de magnitude.

Além de cálculos de fusão, efetuamos também, neste trabalho, cálculos das seções de choque de espalhamento elástico no contexto do modelo de ZPM para os sistemas ${ }^{58} \mathrm{Ni}+{ }^{64} \mathrm{Ni} \mathrm{e}{ }^{16} \mathrm{O}+{ }^{58} \mathrm{Ni}$, ${ }^{208} \mathrm{~Pb}$ em energias em torno da barreira coulombiana. Ao confrontarmos os resultados com dados experimentais pudemos constatar uma boa concordância. Podemos ressaltar que o potencial de polarização obtido é resultado de considerações teóricas de um ponto de vista fundamental, considerando o acoplamento à toda banda vibracional de estados de quadrupolo e octupolo. Para o caso particular do sistema ${ }^{58} \mathrm{Ni}+{ }^{64} \mathrm{Ni}$, com o intuito de comparação, efetuamos também cálculos de CC com o código FRESCO, acoplando porém somente os primeiros estados $2^{+}$e $3^{-}$, devido às limitações numéricas existentes no cálculo de $\mathrm{CC}$ com muitos canais e com acoplamentos a estados muito deformados. Verificamos que, apesar de o modelo de ZPM reproduzir os dados apenas de forma razoável em alguns casos, os resultados de CC são ainda menos satisfatórios, principalmente para ângulos dianteiros e intermediários. Isso se deve, provavelmente, à ausência de acoplamentos a outros canais relevantes.

Um dos objetivos principais deste trabalho foi demonstrar que é possível tratar os canais de espalhamento elástico e de fusão de maneira consistente dentro do mesmo contexto. Claramente, de um ponto de vista teórico a formulação de canais acoplados é a mais adequada para tratar de colisões de íons pesados. Entretanto, devido à complexidade deste problema, qualquer formulação, mesmo a tradicional de CC, sempre introduz algum tipo de simplificação. Mostramos que o cálculo usual de CC pode ter resultados numéricos imprecisos em alguns casos. É importante enfatizar que os modelos GFA e ZPM também estão inseridos no contexto fundamental de canais acoplados, mas as aproximações envolvidas nestes modelos nos permitem obter resultados teóricos precisos em regiões inacessíveis aos cálculos de canais acoplados mais tradicionais. Por outro lado, também mostramos 
que é possível analisar um conjunto muito grande de diferentes sistemas sem utilizar parâmetros ajustáveis. Neste contexto, o potencial de São Paulo emerge como interação bastante adequada ao estudo de colisões entre íons pesados. Obviamente, devido às aproximações assumidas, não é possível esperar um ajuste "perfeito" entre previsões teóricas e dados experimentais. Porém, levando em consideração a ausência de parâmetros ajustáveis e as aproximações assumidas, podemos considerar que a concordância entre nossas previsões teóricas e dados experimentais é muito boa. Mostramos, também, que os ajustes poderiam ser melhorados com a introdução de parâmetros ajustáveis, mas estabelecemos claramente a região realística em que tais parâmetros poderiam variar. 


\section{Apêndice A}

\section{Solução Numérica do Sistema de Equações}

\section{Acopladas}

Neste Apêndice apresentaremos o método utilizado para escrever o programa que resolve o problema de canais acoplados unidimensional descrito na Seção 2.1. O sistema de equações acopladas, obtido a partir da expansão da função de onda total na base de auto-estados internos de oscilador harmônico $|n\rangle$, pode ser representado pela Eq. (2.7):

$$
-\frac{\hbar^{2}}{2 \mu} \frac{d^{2} \psi_{n}(r)}{d r^{2}}+V(r) \psi_{n}(r)+\sum_{m \neq n} V_{n m} \psi_{m}(r)=\left[E-n E^{*}\right] \psi_{n}(r)
$$

onde $V(r)$ é o potencial de espalhamento, assumido na Seção 2.1 como sendo uma barreira parabólica. Cada valor de $n$ representa um canal de espalhamento (elástico se $n=0$ ou inelástico se $n \neq 0$ ) ao qual está associada uma equação diferencial inomogênea acoplada à todas as outras. As condições de contorno são dadas pelas Eqs. (2.9) e (2.10), aqui reproduzidas:

$$
\begin{gathered}
\psi_{n}\left(r \geq r_{0}\right)=\delta_{n, 0} e^{-i k_{0} r}+b_{n} e^{i k_{n} r}, \\
\psi_{n}\left(r \leq-r_{0}\right)=a_{n} e^{-i k_{n} r}
\end{gathered}
$$

indicando que canal elástico, que possui ondas se propagando na direção de $r$ negativo para $r \geq$ $r_{0}$, corresponde a $n=0$. Obviamente, no cálculo numérico, devemos limitar o número total de 
canais a um número finito $n_{\text {máx }}$, assumindo que todos os canais relevantes ao problema estudado estão incluídos no intervalo $0 \leq n \leq n_{\text {máx }}$.

\section{A.1 Resolvendo numericamente uma equação desacoplada}

Inicialmente veremos como uma equação diferencial desacoplada pode ser resolvida numericamente. Uma equação diferencial ordinária de segunda ordem do tipo:

$$
\frac{\mathrm{d}^{2} f(x)}{\mathrm{d} x^{2}}+H(x) f(x)=G(x)
$$

onde $H(x)$ e $G(x)$ também são funções de $x$, pode ser resolvida através do método de Runge-Kutta de segunda ordem, pelo qual temos que:

$$
f(x)=\left[2-h^{2} H(x-h)\right] f(x-h)-f(x-2 h)+h^{2} G(x-h)
$$

sendo que $h$ é o intervalo em $x$ nos quais a função $f(x)$ é calculada. No caso de uma equação análoga à Eq. (A.1), porém desacoplada, temos:

$$
-\frac{\hbar^{2}}{2 \mu} \frac{d^{2} \psi(r)}{d r^{2}}+V(r) \psi(r)=E \psi(r)
$$

com as condições de contorno:

$$
\begin{gathered}
\psi\left(r \geq r_{0}\right)=e^{-i k r}+b e^{i k r} \\
\psi\left(r \leq-r_{0}\right)=a e^{-i k r}
\end{gathered}
$$

Para resolver a Eq. (A.6) podemos usar as Eqs. (A.4) e (A.5). Para isso, no entanto, precisamos conhecer a função de onda em pelo menos dois pontos consecutivos. O comportamento assintótico expresso nas Eqs. (A.7) e (A.8) poderia fornecer tal informação, mas $a$ e $b$ são desconhecidos. Para solucionar este pequeno inconveniente basta lembrar que a Eq. (A.6) é linear. Logo, se $\psi$ é uma solução, $\bar{\psi}=\psi / a$ também é, com condições de contorno:

$$
\bar{\psi}\left(r \geq r_{0}\right)=\frac{1}{a} e^{-i k r}+\frac{b}{a} e^{i k r}
$$




$$
\bar{\psi}\left(r \leq-r_{0}\right)=e^{-i k r}
$$

Como, pela Eq. (A.10), conhecemos o comportamento de $\bar{\psi}$ em pelo menos dois pontos, podemos usar as Eqs. (A.4) e (A.5) escolhendo um valor de $h$ conveniente, para encontrar $\bar{\psi}$ em todo o espaço, incluindo a região de $r \geq r_{0}$. Após a integração, basta comparar o valor da função de onda nos dois últimos pontos obtidos ( $r \geq r_{0}$ ) com os fornecidos pela Eq. (A.9) nos mesmos dois pontos, obtendo assim os valores de $a$ e $b$. Ao invés de usar o valor da função de onda em dois pontos assintóticos, podemos usar também o valor da função de onda em um único ponto e o valor de sua derivada numérica no mesmo ponto, comparando com a Eq. (A.9) e sua derivada.

\section{A.2 Tentativa de generalização para equações acopladas}

Se, de maneira ingênua, tentarmos usar uma generalização direta do método descrito na Seção A.1, veremos que não é possível obter as amplitudes de transmissão e reflexão relativas a todos os canais, representadas por $a_{n}$ e $b_{n}$, respectivamente. Podemos, de maneira análoga, definir funções de onda $\bar{\psi}_{n}=\psi_{n} / a_{n}$ que, devido à linearidade das equações às quais os $\psi_{n}$ obedecem, também é solução da Eq. (A.1). As condições de contorno correspondentes ficam então:

$$
\begin{gathered}
\bar{\psi}_{n}\left(r \geq r_{0}\right)=\frac{\delta_{n, 0}}{a_{n}} e^{-i k_{0} r}+\frac{b_{n}}{a_{n}} e^{i k_{n} r} \\
\bar{\psi}_{n}\left(r \leq-r_{0}\right)=e^{-i k_{n} r}
\end{gathered}
$$

Devido ao termo $\delta_{n, 0}$ na Eq. (A.11), podemos, como no caso da equação desacoplada mostrado na Seção A.1, obter as amplitudes de transmissão e reflexão do canal elástico mas, para os canais inelásticos, o máximo de informação que é possível obter após a integração numérica do sistema de equações acopladas é a razão entre estas amplitudes.

\section{A.3 Solução por inversão de matriz}

Para solucionar o problema descrito na Seção A.2, fazemos uso novamente da linearidade da Eq. (A.1). Uma combinação linear de funções de onda com diferentes condições de contorno que sejam 
soluções da Eq. (A.1) também é uma solução da mesma equação diferencial. Como visto na Seção A.2, o método lá descrito somente possibilita o cálculo das amplitudes de reflexão e transmissão para o canal elástico. Podemos, no entanto, resolver a Eq. (A.1) $n_{\text {máx }}$ vezes com condições de contorno modificadas sendo assumido, em cada uma das vezes, que somente o canal $n=j$ possui amplitude de transmissão diferente de zero, obtendo assim um conjunto de funções de onda cuja combinação linear apropriada fornece a solução com as condições de contorno desejadas, Eqs. (A.2) e (A.3). Consideremos a função $\psi_{n, j}$ que corresponde à solução da Eq. (A.1) para o canal $n$ com o seguinte comportamento assintótico:

$$
\begin{gathered}
\psi_{n, j}\left(r \geq r_{0}\right)=M_{n, j} e^{-i k_{0} r}+N_{n, j} e^{i k_{n} r}, \\
\psi_{n, j}\left(r \leq-r_{0}\right)=\delta_{n, j} e^{-i k_{n} r}
\end{gathered}
$$

Pela definição das condições de contorno acima descritas podemos integrar numericamente os $\psi_{n, j}$ a partir de dois pontos na região de $r \leq-r_{0}$, utilizando as Eqs. (A.4) e (A.5) de forma apropriada, obtendo os elementos de matriz $M_{n, j}$ e $N_{n, j}$. Se, por exemplo, $r_{f}$ for o ponto final de integração onde $\psi_{n, j}$ foi calculado e $h$ o passo de integração numérica utilizado, temos que:

$$
\begin{gathered}
M_{n, j}=\frac{e^{i k_{n} r_{f}}}{2 i \sin \left(k_{n} h\right)}\left[\psi_{n, j}\left(r_{f}-h\right)-e^{-i k_{n} h} \psi_{n, j}\left(r_{f}\right)\right] \\
N_{n, j}=\frac{e^{-i k_{n} r_{f}}}{2 i \sin \left(k_{n} h\right)}\left[e^{i k_{n} h} \psi_{n, j}\left(r_{f}\right)-\psi_{n, j}\left(r_{f}-h\right)\right]
\end{gathered}
$$

onde tanto $r_{f}$ quanto $r_{f}-h$ correspondem a pontos assintóticos pertencentes à região $r \geq r_{0}$. Se ao invés de utilizarmos o valor da função de onda em dois pontos, calcularmos o valor da derivada numérica $\psi_{n, j}^{\prime}$ no ponto $r_{f}$, obtemos:

$$
\begin{aligned}
& M_{n, j}=\frac{e^{i k_{n} r_{f}}}{2}\left[\psi_{n, j}\left(r_{f}\right)+\frac{i}{k_{n}} \psi_{n, j}^{\prime}\left(r_{f}\right)\right] \\
& N_{n, j}=\frac{e^{-i k_{n} r_{f}}}{2}\left[\psi_{n, j}\left(r_{f}\right)-\frac{i}{k_{n}} \psi_{n, j}^{\prime}\left(r_{f}\right)\right]
\end{aligned}
$$


Comparando as condições de contorno utilizadas dadas pelas Eqs. (A.13) e (A.14) com as do problema original, representadas pelas Eq. (A.2) e (A.3), temos que as amplitudes de transmissão podem ser obtidas resolvendo o sistema linear de equações dado por:

$$
\sum_{j=0}^{n_{\operatorname{máx}}} a_{j} M_{n, j}=\delta_{0, n}
$$

Tendo resolvido o sistema linear representado pela Eq. (A.19) pela inversão da matriz formada pelos elementos $M_{n, j}$ ou por qualquer outro método, podemos obter as amplitudes de reflexão simplesmente por:

$$
b_{n}=\sum_{j=0}^{n_{\text {máx }}} a_{n} N_{n, j}
$$




\section{Apêndice B}

\section{Dedução Analítica das Projeções $U_{m n}$}

Na Seção 2.4, no decorrer do desenvolvimento do modelo de Movimento de Ponto Zero, foram utilizadas projeções de auto-estados de oscilador harmônico de uma variável em outra. Os elementos de matriz $U_{m n}$ foram definidos na Eq. (2.50). Neste Apêndice deduziremos analiticamente a Eq. (2.50).

A Hamiltoniana de oscilador harmônico na coordenada $s$ pode ser escrita como:

$$
H_{o s c}(s)=-E^{*}\left[\sigma^{2} \frac{\partial^{2}}{\partial s^{2}}-\frac{s^{2}}{4 \sigma^{2}}\right]
$$

onde a energia de excitação, $E^{*}$, e o parâmetro de massa do oscilador, $D$, se relacionam com o desvio padrão de vibração no estado fundamental, $\sigma$, através de $D=\hbar^{2} / 2 \sigma^{2} E^{*}$. O auto-estado correspondente $|n(s)\rangle$ satisfaz a equação de Schrödinger interna:

$$
H_{o s c}(s)|n(s)\rangle=\left(n+\frac{1}{2}\right) E^{*}|n(s)\rangle
$$

A forma explícita da auto-função $|n(s)\rangle$ na representação de Schrödinger é:

$$
|n(s)\rangle=\frac{1}{\left(2 \pi \sigma^{2}\right)^{1 / 4}} e^{-s^{2} / 4 \sigma^{2}} \frac{H_{n}(s / \sigma \sqrt{2})}{\sqrt{n ! 2^{n}}}
$$

onde $H_{n}(s / \sigma \sqrt{2})$ são os polinômios de Hermite de ordem $\mathrm{n}$.

Se definirmos a coordenada $u=s-2 \frac{F \sigma}{E^{*}}$, onde $F$ é a constante de acoplamento linear à barreira 
parabólica descrita na Seção 2.4, podemos escrever expressões análogas às Eqs. (B.1) a (B.3), porém, na coordenada $u$ :

$$
\begin{gathered}
H_{o s c}(u)=-E^{*}\left[\sigma^{2} \frac{\partial^{2}}{\partial u^{2}}-\frac{u^{2}}{4 \sigma^{2}}\right] \\
H_{o s c}(u)|n(u)\rangle=\left(n+\frac{1}{2}\right) E^{*}|n(u)\rangle \\
|n(u)\rangle=\frac{1}{\left(2 \pi \sigma^{2}\right)^{1 / 4}} e^{-u^{2} / 4 \sigma^{2}} \frac{H_{n}(u / \sigma \sqrt{2})}{\sqrt{n ! 2^{n}}}
\end{gathered}
$$

Como relações de ortonormalidade das auto-funções de oscilador harmônico temos, obviamente, $\langle n(s) \mid m(s)\rangle=\delta_{n, m} \mathrm{e}\langle n(u) \mid m(u)\rangle=\delta_{n, m}$.

As projeções $U_{m n} \equiv\langle m(u) \mid n(s)\rangle$ podem ser calculadas explicitamente usando as Eqs. (B.3) e (B.6), através da integral:

$$
U_{m n}=\int \frac{1}{\left(2 \pi \sigma^{2}\right)^{1 / 4}} e^{-u^{2} / 4 \sigma^{2}} \frac{H_{m}(u / \sigma \sqrt{2})}{\sqrt{m ! 2^{m}}} \frac{1}{\left(2 \pi \sigma^{2}\right)^{1 / 4}} e^{-s^{2} / 4 \sigma^{2}} \frac{H_{n}(s / \sigma \sqrt{2})}{\sqrt{n ! 2^{n}}} \mathrm{~d} s
$$

Temos, na Eq. (B.7), um produto de polinômios de Hermite de diferentes ordens e em coordenadas diferentes, uma transladada da outra por uma constante. Um polinômio de Hermite de ordem $n$ na coordenada $x+a$, onde $a$ é uma constante, pode, pela própria definição, ser expresso como:

$$
H_{n}(x+a)=(-1)^{n} e^{(x+a)^{2}}\left(\frac{\mathrm{d}}{\mathrm{d}(x+a)}\right)^{n} e^{-(x+a)^{2}}
$$

Podemos reescrever a Eq. (B.8) como:

$$
\begin{aligned}
H_{n}(x+a) & =(-1)^{n} e^{x^{2}} e^{2 a x} \sum_{j=0}^{n}\left[\left(\begin{array}{c}
n \\
j
\end{array}\right) \frac{\mathrm{d}^{j}}{\mathrm{~d} x^{j}} e^{-2 a x} \frac{\mathrm{d}^{n-j}}{\mathrm{~d} x^{n-j}} e^{-x^{2}}\right] \\
& =(-1)^{n} e^{x^{2}} e^{2 a x} \sum_{j=0}^{n}\left[\frac{n !}{j !(n-j) !}(-2 a)^{j} e^{-2 a x} \frac{\mathrm{d}^{n-j}}{\mathrm{~d} x^{n-j}} e^{-x^{2}}\right] \\
& =\sum_{j=0}^{n} \frac{n !(-2 a)^{j}}{j !(n-j) !} \frac{(-1)^{n}}{(-1)^{n-j}}(-1)^{n-j} e^{x^{2}} \frac{\mathrm{d}^{n-j}}{\mathrm{~d} x^{n-j}} e^{-x^{2}} \\
& =\sum_{j=0}^{n} \frac{n !(2 a)^{j}}{j !(n-j) !} H_{n-j}(x)
\end{aligned}
$$


sendo que os termos $\left(\begin{array}{l}n \\ j\end{array}\right)$ representam os coeficientes do binômio de Newton. Na última passagem da Eq. (B.9), foi usada a definição do polinômio de Hermite, dada pela Eq. (B.8) com $a=0$. Trocando $n-j$ por $j$ e vice-versa, temos, finalmente:

$$
H_{n}(x+a)=\sum_{j=0}^{n} \frac{n !(2 a)^{n-j}}{j !(n-j) !} H_{j}(x)
$$

$\mathrm{Na}$ Eq. (B.7) podemos encontrar um produto de duas gaussianas com diferentes valores médios, uma centrada em $s$ e outra em $u=s-2 \frac{F \sigma}{E^{*}}$. Podemos, através de mudanças de variáveis, transformar este produto em uma única gaussiana:

$$
\begin{aligned}
e^{-u^{2} / 4 \sigma^{2}} e^{-s^{2} / 4 \sigma^{2}} \mathrm{~d} s & =\exp \left\{-\frac{1}{4 \sigma^{2}}\left[2 u^{2}+\frac{4 F^{2} \sigma^{4}}{E^{* 2}}+\frac{4 F \sigma^{2}}{E^{*}} u\right]\right\} \mathrm{d} u \\
& =\exp \left\{-\frac{1}{2 \sigma^{2}}\left(u+\frac{F \sigma^{2}}{E^{*}}\right)^{2}-\frac{1}{2} \frac{F^{2} \sigma^{2}}{E^{* 2}}\right\} \mathrm{d} u \\
& =e^{-v^{2} / 2 \sigma^{2}} e^{-\frac{1}{2}\left(\frac{F \sigma}{E^{*}}\right)^{2}} \mathrm{~d} v
\end{aligned}
$$

onde $v=u+\frac{F \sigma^{2}}{E^{*}}$.

Substituindo a Eq. (B.11) na Eq. (B.7) e fazendo as correspondentes mudanças de variáveis para a coordenada $v$ temos:

$$
U_{m n}=e^{-\frac{1}{2}\left(\frac{F \sigma}{E^{*}}\right)^{2}} \int \frac{1}{\left(2 \pi \sigma^{2}\right)^{1 / 2}} \frac{H_{m}\left(\frac{v}{\sigma \sqrt{2}}-\frac{F \sigma}{E^{*} \sqrt{2}}\right)}{\sqrt{m ! 2^{m}}} \frac{H_{m}\left(\frac{v}{\sigma \sqrt{2}}+\frac{F \sigma}{E^{*} \sqrt{2}}\right)}{\sqrt{m ! 2^{m}}} e^{-v^{2} / 2 \sigma^{2}} \mathrm{~d} v
$$

Comparando os polinômios de Hermite da Eq. (B.12) com a Eq. (B.10), fazendo as atribuições apropriadas a $x$ e $a$, usando a definição da auto-função de oscilador harmônico dada pela Eq. (B.3), na coordenada $v$, e lembrando das condições de ortonormalidade entre estas funções, obtemos:

$$
U_{m n}=e^{-\frac{1}{2}\left(\frac{F \sigma}{E^{*}}\right)^{2}} \sum_{j=0}^{m} \sum_{j^{\prime}=0}^{n} \sqrt{\frac{m ! n !}{j ! j^{\prime} !}}\left(\frac{F \sigma}{E^{*}}\right)^{m-j+n-j^{\prime}} \frac{(-1)^{m-j}}{(m-j) !\left(n-j^{\prime}\right) !} \delta_{j, j^{\prime}}
$$


Efetuando a soma sobre o índice $j^{\prime}$, obtemos, finalmente:

$$
U_{m n}=e^{-\frac{1}{2}\left(\frac{F \sigma}{E^{*}}\right)^{2}} \sum_{j=0}^{\min (n, m)}(-1)^{m-j} \frac{\sqrt{m ! n !}\left(\frac{F \sigma}{E^{*}}\right)^{m+n-2 j}}{j !(m-j) !(n-j) !}
$$

Esta é justamente a Eq. (2.50) apresentada na Seção 2.4.

Podemos ainda reescrever a Eq. (B.14) em função de $U_{m 0}$ :

$$
U_{m n}=U_{m 0} \sum_{j=0}^{\min (n, m)} \frac{(-1)^{j} \sqrt{n !}}{j !(n-j) !} \prod_{l=0}^{j-1}(m-l)\left(\frac{F \sigma}{E^{*}}\right)^{n-2 j}
$$

onde $U_{m 0}$, pela Eq. (B.14), é dado por:

$$
U_{m 0}=e^{-\frac{1}{2}\left(\frac{F \sigma}{E^{*}}\right)^{2}} \frac{\left(\frac{F \sigma}{E^{*}}\right)^{m}}{\sqrt{m !}}
$$

Como vimos na Seção 2.4, os produtos de projeções $U_{m 0}^{2}$ e $U_{m 1} U_{m 0}$ são particularmente importantes e podem ser escritos, pelas Eqs. (B.15) e (B.16) como:

$$
\begin{gathered}
U_{m 0}^{2}=e^{-\left(\frac{F \sigma}{E^{*}}\right)^{2}} \frac{\left(\frac{F \sigma}{E^{*}}\right)^{2 m}}{m !} \\
U_{m 1} U_{m 0}=U_{m 0}^{2}\left[\left(\frac{F \sigma}{E^{*}}\right)-\frac{m}{\left(\frac{F \sigma}{E^{*}}\right)}\right]
\end{gathered}
$$

Vemos que a Eq. (B.17) corresponde a uma distribuição de Poisson na variável $m$ com valor médio $\langle m\rangle=\left(\frac{F \sigma}{E^{*}}\right)^{2}$ e desvio padrão $\sigma_{m}=\sqrt{\langle m\rangle}$. Para valores muito grandes de $\langle m\rangle$, a distribuição de Poisson se aproxima de uma distribuição gaussiana que, tendo o mesmo valor médio, mesmo desvio padrão e sendo normalizada, pode ser escrita como:

$$
\begin{aligned}
U_{m 0}^{2} & =\frac{1}{\sqrt{2 \pi \sigma_{m}^{2}}} e^{-\frac{(m-\langle m\rangle)^{2}}{2 \sigma_{m}^{2}}} \\
& =\frac{E^{*}}{F \sigma \sqrt{2 \pi}} e^{-\frac{m-\left(\frac{F \sigma}{E^{*}}\right)^{2}}{2\left(\frac{F \sigma}{E^{*}}\right)^{2}}}
\end{aligned}
$$




\section{Apêndice C}

\section{Autofunção Analítica por Séries de uma}

\section{Partícula através de uma Barreira Parabólica}

Consideremos a equação de Schrödinger:

$$
-\frac{\hbar^{2}}{2 \mu} \frac{d^{2} \bar{Y}(r)}{d r^{2}}+\left[V_{B 0}-\frac{1}{2} \mu \omega^{2} r^{2}\right] \bar{Y}(r)=E \bar{Y}(r)
$$

com as seguintes condições de contorno:

$$
\begin{gathered}
\bar{Y}\left(r \geq r_{0}\right)=e^{-i k r}+B e^{i k r}, \\
\bar{Y}\left(r \leq-r_{0}\right)=A e^{-i k r},
\end{gathered}
$$

com $E=\frac{\hbar^{2} k^{2}}{2 \mu}$ e $r_{0}$ definido por $\frac{1}{2} \mu \omega^{2} r_{0}^{2}=V_{B 0}$. Logo, a altura da barreira $V_{B}$ é um deslocamento da altura original $V_{B 0}, V_{B}=V_{B 0}+\Delta V_{B}$. Temos, pelas Eqs. (C.2) e (C.3), que o potencial é nulo para $|r| \geq r_{0}$

Definindo $x=\sqrt{\frac{2 \mu \omega}{\hbar}} r, y=\frac{2 \mu \omega}{\hbar} \bar{Y}$ e $a=\frac{V_{B 0}-E}{\hbar \omega}$ obtém-se:

$$
\frac{d^{2} y}{d x^{2}}+\left(\frac{1}{4} x^{2}-a\right) y(x)=0
$$


A Eq. (C.4) corresponde à função cilíndrica parabólica, que pode ser escrita como [122]:

$$
y(x)=C_{P} Y_{P}(x)+C_{I} Y_{I}(x)
$$

onde as funções par e ímpar $Y_{P}$ e $Y_{I}$ são dadas pelas seguintes séries:

$$
\begin{aligned}
& Y_{P}(x)=\sum_{j=0}^{\Delta j=2} a_{j} \frac{x^{j}}{j !}, \\
& Y_{I}(x)=\sum_{j=1}^{\Delta j=2} a_{j} \frac{x^{j}}{j !}
\end{aligned}
$$

$\operatorname{com} a_{0}=1, a_{1}=1, a_{2}=a, a_{3}=a, a_{j+2}=a a_{j}-\frac{1}{4} j(j-1) a_{j-2}$.

As Eqs. (C.2) e (C.3), e suas correspondentes derivadas, podem ser reescritas em termos de $y$ :

$$
\begin{gathered}
C_{P} Y_{P}\left(x_{0}\right)+C_{I} Y_{I}\left(x_{0}\right)=\frac{2 \mu \omega}{\hbar}\left[e^{-2 i \sqrt{E V_{B 0}} / \hbar \omega}+B e^{2 i \sqrt{E V_{B 0}} / \hbar \omega}\right], \\
C_{P} Y_{P}\left(-x_{0}\right)+C_{I} Y_{I}\left(-x_{0}\right)=\frac{2 \mu \omega}{\hbar} A e^{2 i \sqrt{E V_{B 0}} / \hbar \omega} \\
C_{P} Y_{P}^{\prime}\left(x_{0}\right)+C_{I} Y_{I}^{\prime}\left(x_{0}\right)=i \frac{2 \mu \sqrt{E \hbar \omega}}{\hbar^{2}}\left[B e^{2 i \sqrt{E V_{B 0}} / \hbar \omega}-e^{-2 i \sqrt{E V_{B 0}} / \hbar \omega}\right], \\
C_{P} Y_{P}^{\prime}\left(-x_{0}\right)+C_{I} Y_{I}^{\prime}\left(-x_{0}\right)=-i \frac{2 \mu \sqrt{E \hbar \omega}}{\hbar^{2}} A e^{2 i \sqrt{E V_{B 0}} / \hbar \omega},
\end{gathered}
$$

onde $Y^{\prime}$ é a derivada de $Y$ em relação a $x$ e $x_{0}=2 \sqrt{V_{B 0} / \hbar \omega} \cdot C_{P}, C_{I}, A$ e $B$ podem ser extraídos deste conjunto de equações para obter a função de onda na região $-r_{0} \leq r \leq r_{0}$ :

$$
\begin{gathered}
C_{P}=\frac{2 \mu \omega}{\hbar} \sqrt{\frac{E}{\hbar \omega}} \frac{e^{-2 i \sqrt{E V_{B 0}} / \hbar \omega}}{i Y_{P}^{\prime}\left(x_{0}\right)+\sqrt{\frac{E}{\hbar \omega}} Y_{P}\left(x_{0}\right)} \\
C_{I}=\frac{2 \mu \omega}{\hbar} \sqrt{\frac{E}{\hbar \omega}} \frac{e^{-2 i \sqrt{E V_{B 0}} / \hbar \omega}}{i Y_{I}^{\prime}\left(x_{0}\right)+\sqrt{\frac{E}{\hbar \omega}} Y_{I}\left(x_{0}\right)} \\
A=e^{-4 i \sqrt{E V_{B 0}} / \hbar \omega} \sqrt{\frac{E}{\hbar \omega}}\left[\frac{Y_{P}\left(x_{0}\right)}{i Y_{P}^{\prime}\left(x_{0}\right)+\sqrt{\frac{E}{\hbar \omega}} Y_{P}\left(x_{0}\right)}-\frac{Y_{I}\left(x_{0}\right)}{i Y_{I}^{\prime}\left(x_{0}\right)+\sqrt{\frac{E}{\hbar \omega}} Y_{I}\left(x_{0}\right)}\right]
\end{gathered}
$$




$$
\begin{gathered}
B=e^{-4 i \sqrt{E V_{B 0}} / \hbar \omega}\left\{\sqrt{\frac{E}{\hbar \omega}}\left[\frac{Y_{P}\left(x_{0}\right)}{i Y_{P}^{\prime}\left(x_{0}\right)+\sqrt{\frac{E}{\hbar \omega}} Y_{P}\left(x_{0}\right)}+\frac{Y_{I}\left(x_{0}\right)}{i Y_{I}^{\prime}\left(x_{0}\right)+\sqrt{\frac{E}{\hbar \omega}} Y_{I}\left(x_{0}\right)}\right]-1\right\} \\
\bar{Y}(r)=e^{-2 i \sqrt{E V_{B 0}} / \hbar \omega} \sqrt{\frac{E}{\hbar \omega}}\left[\frac{Y_{P}(x)}{i Y_{P}^{\prime}\left(x_{0}\right)+\sqrt{\frac{E}{\hbar \omega}} Y_{P}\left(x_{0}\right)}+\frac{Y_{I}(x)}{i Y_{I}^{\prime}\left(x_{0}\right)+\sqrt{\frac{E}{\hbar \omega}} Y_{I}\left(x_{0}\right)}\right] .
\end{gathered}
$$

Calculando $|A|^{2}$ pela Eq. (C.14), temos que o coeficiente de transmissão $T=|A|^{2}$ é dado por:

$$
T=\frac{\left[Y_{I}\left(x_{0}\right) Y_{P}^{\prime}\left(x_{0}\right)-Y_{P}\left(x_{0}\right) Y_{I}^{\prime}\left(x_{0}\right)\right]^{2}}{\left[\frac{\hbar \omega}{E} Y_{I}^{\prime 2}\left(x_{0}\right)+Y_{I}^{2}\left(x_{0}\right)\right]\left[\frac{\hbar \omega}{E} Y_{P}^{\prime 2}\left(x_{0}\right)+Y_{P}^{2}\left(x_{0}\right)\right]} .
$$

Ao calcularmos o coeficiente de reflexão $R=|B|^{2}$ pela Eq. (C.15) obtemos, como esperado pela conservação de fluxo, $R+T=1$.

Nos cálculos realizados no Capítulo 2, aproximadamente duzentos termos termos foram incluídos nas séries para $Y_{P}$ e $Y_{I}$ (e suas derivadas) de forma a obter boa convergência numérica para a função de onda. No entanto, é passível de advertência que, apesar das séries representarem a função de onda exata, do ponto de vista numérico é difícil obter resultados precisos em alguns casos onde $\left|V_{B 0}-E\right| \gg \hbar \omega$. Nestes casos, porém, se continuarmos procurando soluções analíticas, podemos utilizar as aproximações WKB, apresentadas no Apêndice D. 


\section{Apêndice D}

\section{Aproximação WKB para uma Partícula através de uma Barreira Parabólica}

Consideremos a equação de Schrödinger, para $|r| \leq r_{0}$ :

$$
-\frac{\hbar^{2}}{2 \mu} \frac{d^{2} \psi(r)}{d r^{2}}+\left[V_{B}-\frac{1}{2} \mu \omega^{2} r^{2}\right] \psi(r)=E \psi(r),
$$

com as seguintes condições de contorno:

$$
\begin{gathered}
\psi\left(r \geq r_{0}\right)=e^{-i k r}+B e^{i k r}, \\
\psi\left(r \leq-r_{0}\right)=A e^{-i k r}
\end{gathered}
$$

com $E=\frac{\hbar^{2} k^{2}}{2 \mu}$ e $r_{0}$ definido por $\frac{1}{2} \mu \omega^{2} r_{0}^{2}=V_{B 0}$. Logo, a altura da barreira $V_{B}$ é um deslocamento da altura original $V_{B 0}, V_{B}=V_{B 0}+\Delta V_{B}$. Temos, pelas Eqs. (D.2) e (D.3), que o potencial é nulo para $|r| \geq r_{0}$. Mostraremos nas seções seguintes como foram obtidas as funções de onda, soluções da Eq. (D.1), através da aproximação semi-clássica de Wentzel-Kramers-Brillouin (WKB) para os três casos que resultam considerando os diferentes valores possíveis de $E, V_{B}$ e $V_{B 0}$. Nos desenvolvimentos apresentados nas próximas seções, foram usadas as formulações WKB nas formas como expostas na Ref. [123]. 


\section{D.1 Primeiro Caso: $E \gg V_{B}$}

No caso em que a energia cinética assintótica é maior que a altura da barreira, temos que a função de onda na região em que $|r| \leq r_{0}$, segundo a aproximação WKB, pode ser descrita como:

$$
\psi(r)=C_{+} \sqrt{\frac{\lambda(r)}{2 \pi}} e^{2 \pi i \int_{r_{0}}^{r} \frac{\mathrm{d} x}{\lambda(x)}}+C_{-} \sqrt{\frac{\lambda(r)}{2 \pi}} e^{-2 \pi i \int_{r_{0}}^{r} \frac{\mathrm{d} x}{\lambda(x)}}
$$

onde $C_{+}$e $C_{-}$são constantes e $\lambda(r)$ é o comprimento de onda local:

$$
\lambda(r)=\frac{2 \pi \hbar}{\sqrt{2 \mu\left(E-V_{B}+\frac{1}{2} \mu \omega^{2} r^{2}\right)}}
$$

Comparando as Eqs. (D.2) e (D.3) com a Eq. (D.4) e aplicando as condições de continuidade da função de onda e de sua derivada nos pontos $\pm r_{0}$, obtemos o sistema de equações:

$$
\begin{gathered}
C_{+}+C_{-}=\frac{\left[2 \mu\left(E-V_{B}+V_{B 0}\right)\right]^{1 / 4}}{\sqrt{\hbar}}\left[e^{-i k r_{0}}+B e^{i k r_{0}}\right] \\
-i k e^{-i k r_{0}}+i k B e^{i k r_{0}}=-\frac{\hbar \omega}{4} \frac{\sqrt{V_{B 0}}}{\sqrt{\hbar}} \frac{\left[2 \mu\left(E-V_{B}+V_{B 0}\right)\right]^{1 / 4}}{\left(E-V_{B}+V_{B 0}\right)^{3 / 2}}\left[C_{+}+C_{-}\right]+ \\
+i \frac{\left[2 \mu\left(E-V_{B}+V_{B 0}\right)\right]^{1 / 4}}{\sqrt{\hbar}}\left[C_{+}-C_{-}\right] \\
C_{+} e^{i \phi}+C_{-} e^{-i \phi}=\frac{\left[2 \mu\left(E-V_{B}+V_{B 0}\right)\right]^{1 / 4}}{\sqrt{\hbar}} A e^{i k r_{0}} \\
-i k A e^{i k r_{0}}=\frac{\hbar \omega}{4} \frac{\sqrt{V_{B 0}}}{\sqrt{\hbar}} \frac{\left[2 \mu\left(E-V_{B}+V_{B 0}\right)\right]^{1 / 4}}{\left(E-V_{B}+V_{B 0}\right)^{3 / 2}}\left[C_{+} e^{i \phi}+C_{-} e^{-i \phi}\right]+ \\
+i \frac{\left[2 \mu\left(E-V_{B}+V_{B 0}\right)\right]^{1 / 4}}{\sqrt{\hbar}}\left[C_{+} e^{i \phi}-C_{-} e^{-i \phi}\right]
\end{gathered}
$$

onde a fase $\phi$, que representa a integral do inverso do comprimento de onda local em toda a região da 
barreira, é dada por:

$$
\begin{aligned}
\phi & =2 \pi \int_{r_{0}}^{-r_{0}} \frac{\mathrm{d} x}{\lambda(x)} \\
& =\frac{E-V_{B}}{\hbar \omega} \ln \left[\frac{\sqrt{E-V_{B}+V_{B 0}}-\sqrt{V_{B 0}}}{\sqrt{E-V_{B}+V_{B 0}}+\sqrt{V_{B 0}}}\right]-2 \frac{\sqrt{V_{B 0}\left(E-V_{B}+V_{B 0}\right)}}{\hbar \omega}
\end{aligned}
$$

Resolvendo o sistema representado pelas Eqs. (D.6), (D.7), (D.8) e (D.9), obtemos, finalmente:

$$
\begin{aligned}
C_{+}= & -\frac{8 i(2 \mu)^{1 / 4}}{\sqrt{\hbar}} \sqrt{E}\left(E-V_{B}+V_{B 0}\right)^{5 / 4} e^{-2 i \frac{\sqrt{E V_{B 0}}}{\hbar \omega}} \times \\
& \times\left[\hbar \omega \sqrt{V_{B 0}}+4 i\left(E-V_{B}+V_{B 0}\right)\left(\sqrt{E}-\sqrt{E-V_{B}+V_{B 0}}\right)\right] \times \\
& \times\left\{e^{2 i \phi}\left[\hbar \omega \sqrt{V_{B 0}}+4 i\left(E-V_{B}+V_{B 0}\right)\left(\sqrt{E}+\sqrt{E-V_{B}+V_{B 0}}\right)\right]^{2}+\right. \\
& \left.-\left[\hbar \omega \sqrt{V_{B 0}}+4 i\left(E-V_{B}+V_{B 0}\right)\left(\sqrt{E}-\sqrt{E-V_{B}+V_{B 0}}\right)\right]^{2}\right\}^{-1} \\
& C_{-}=-C_{+} e^{2 i \phi} \frac{\hbar \omega \sqrt{V_{B 0}}+4 i\left(E-V_{B}+V_{B 0}\right)\left(\sqrt{E}+\sqrt{E-V_{B}+V_{B 0}}\right)}{\hbar V_{B 0}+4 i\left(E-V_{B}+V_{B 0}\right)\left(\sqrt{E}-\sqrt{E-V_{B}+V_{B 0}}\right)} \\
A= & -64 \sqrt{E}\left(E-V_{B}+V_{B 0}\right)^{5 / 2} e^{-4 i \frac{\sqrt{E V_{B 0}}}{\hbar \omega}} \times \\
& \times\left\{e^{i \phi}\left[\hbar \omega \sqrt{V_{B 0}}+4 i\left(E-V_{B}+V_{B 0}\right)\left(\sqrt{E}+\sqrt{E-V_{B}+V_{B 0}}\right)\right]^{2}+\right. \\
& \left.-e^{-i \phi}\left[\hbar \omega \sqrt{V_{B 0}}+4 i\left(E-V_{B}+V_{B 0}\right)\left(\sqrt{E}-\sqrt{E-V_{B}+V_{B 0}}\right)\right]^{2}\right\}^{-1}
\end{aligned}
$$

A amplitude de reflexão $B$ pode ser encontrada substituindo-se as Eqs. (D.11) e (D.12) na Eq. (D.6).

\section{D.2 Segundo Caso: $E \ll V_{B} ; E<V_{B}-V_{B 0}$}

Quando a energia cinética assintótica é menor que a altura da barreira e temos que $E<V_{B}-V_{B 0}$, a partícula atravessa somente regiões classicamente proibidas durante a interação com o potencial 
parabólico. Neste caso, a aproximação WKB para a função de onda pode ser escrita como:

$$
\psi(r)=C_{+} \sqrt{\frac{l(r)}{2 \pi}} e^{2 \pi \int_{r_{0}}^{r} \frac{\mathrm{d} x}{l(x)}}+C_{-} \sqrt{\frac{l(r)}{2 \pi}} e^{-2 \pi \int_{r_{0}}^{r} \frac{\mathrm{d} x}{l(x)}}
$$

onde $C_{+}$e $C_{-}$são constantes e $l(r)$ é dado por:

$$
l(r)=\frac{2 \pi \hbar}{\sqrt{2 \mu\left(V_{B}-E-\frac{1}{2} \mu \omega^{2} r^{2}\right)}}
$$

Comparando as Eqs. (D.2) e (D.3) com a Eq. (D.14) e aplicando as condições de continuidade da função de onda e de sua derivada nos pontos $\pm r_{0}$, obtemos os sistema de equações:

$$
\begin{gathered}
e^{-i k r_{0}}+B e^{i k r_{0}}=\frac{\sqrt{\hbar}\left[C_{+}+C_{-}\right]}{\left[2 \mu\left(V_{B}-E-V_{B 0}\right)\right]^{1 / 4}} \\
i k B e^{i k r_{0}}-i k e^{-i k r_{0}}=\frac{\hbar \omega}{4} \frac{\sqrt{V_{B 0}}}{\sqrt{\hbar}} \frac{\left[2 \mu\left(V_{B}-E-V_{B 0}\right)\right]^{1 / 4}}{\left(V_{B}-E-V_{B 0}\right)^{3 / 2}}\left[C_{+}+C_{-}\right]+ \\
+\frac{\left[2 \mu\left(V_{B}-E-V_{B 0}\right)\right]^{1 / 4}}{\sqrt{\hbar}}\left[C_{+}-C_{-}\right] \\
A e^{i k r_{0}}=\left[C_{+} e^{\phi}+C_{-} e^{-\phi}\right] \frac{\sqrt{\hbar}}{\left[2 \mu\left(V_{B}-E-V_{B 0}\right)\right]^{1 / 4}} \\
-i k A e^{i k r_{0}}=-\frac{\hbar \omega}{4} \frac{\sqrt{V_{B 0}}}{\sqrt{\hbar}} \frac{\left[2 \mu\left(V_{B}-E-V_{B 0}\right)\right]^{1 / 4}}{\left(V_{B}-E-V_{B 0}\right)^{3 / 2}}\left[C_{+} e^{\phi}+C_{-} e^{-\phi}\right]+ \\
+\frac{\left[2 \mu\left(V_{B}-E-V_{B 0}\right)\right]^{1 / 4}}{\sqrt{\hbar}}\left[C_{+} e^{\phi}-C_{-} e^{-\phi}\right]
\end{gathered}
$$

onde $\phi$, que representa a integral de $l(x)$ sob a região da barreira, é dado por:

$$
\phi=2 \pi \int_{r_{0}}^{-r_{0}} \frac{\mathrm{d} x}{l(x)}=-\frac{2}{\hbar \omega}\left[\sqrt{V_{B 0}\left(V_{B}-E-V_{B 0}\right)}+\left(V_{B}-E\right) \arcsin \left(\sqrt{\frac{V_{B 0}}{V_{B}-E}}\right)\right]
$$

Resolvendo o sistema representado pelas Eqs. (D.16), (D.17), (D.18) e (D.19), obtemos, finalmente: 


$$
\begin{aligned}
C_{-}= & \frac{8 i(2 \mu)^{1 / 4}}{\sqrt{\hbar}} \sqrt{E}\left(V_{B}-E-V_{B 0}\right)^{5 / 4} e^{-2 i \frac{\sqrt{E V_{B 0}}}{\hbar \omega} \times} \\
& \times\left[4\left(V_{B}-E-V_{B 0}\right)\left(i \sqrt{E}+\sqrt{V_{B}-E-V_{B 0}}\right)-\hbar \omega \sqrt{V_{B 0}}\right] \times \\
& \times\left\{\left[4\left(V_{B}-E-V_{B 0}\right)\left(i \sqrt{E}+\sqrt{V_{B}-E-V_{B 0}}\right)-\hbar \omega \sqrt{V_{B 0}}\right]^{2}+\right. \\
& \left.-e^{-2 \phi}\left[4\left(V_{B}-E-V_{B 0}\right)\left(i \sqrt{E}-\sqrt{V_{B}-E-V_{B 0}}\right)-\hbar \omega \sqrt{V_{B 0}}\right]^{2}\right\}^{-1} \\
C_{+}= & -C_{-} e^{-2 \phi} \frac{4\left(V_{B}-E-V_{B 0}\right)\left(i \sqrt{E}-\sqrt{V_{B}-E-V_{B 0}}\right)-\hbar \omega \sqrt{V_{B 0}}}{4\left(V_{B}-E-V_{B 0}\right)\left(i \sqrt{E}+\sqrt{V_{B}-E-V_{B 0}}\right)-\hbar \omega \sqrt{V_{B 0}}}
\end{aligned}
$$

As amplitudes de transmissão $A$ e reflexão $B$ podem ser encontradas substituindo-se as Eqs. (D.21) e (D.22) nas Eq. (D.18) e (D.16).

\section{D.3 Terceiro Caso: $E \ll V_{B} ; E>V_{B}-V_{B 0}$}

Se a energia cinética assintótica é menor que a altura da barreira e temos que $E>V_{B}-V_{B 0}$, a partícula atravessa regiões classicamente proibidas e permitidas durante a interação com o potencial parabólico. Podemos então, neste caso, dividir a função de onda em cinco regiões diferentes:

- Região 1: $r \leq-r_{0}$. Nesta região assintótica de potencial nulo a função de onda $\psi_{1}$ é dada pela Eq. (D.3).

- Região 2: $-r_{0} \leq r \leq r_{1}$, onde $r_{1}$ é o ponto de retorno para valores negativos de $r$, ou seja, $r_{1}=-\sqrt{\frac{2\left(V_{B}-E\right)}{\mu \omega^{2}}}$. Nesta região, a partícula está sujeita ao potencial parabólico mas este é menor do que a energia cinética assintótica. A função de onda, pela aproximação WKB, pode então ser dada nesta região por uma expressão análoga à Eq. (D.4):

$$
\psi_{2}(r)=C_{2-} \sqrt{\frac{\lambda(r)}{2 \pi}} e^{2 \pi i \int_{r}^{r_{1}} \frac{\mathrm{d} x}{\lambda(x)}+i \pi / 4}+C_{2+} \sqrt{\frac{\lambda(r)}{2 \pi}} e^{-2 \pi i \int_{r}^{r_{1}} \frac{\mathrm{d} x}{\lambda(x)}-i \pi / 4}
$$

onde $C_{2-}$ e $C_{2+}$ são constantes e $\lambda(r)$ é o comprimento de onda local dado pela Eq. (D.5). 
- Região 3: $r_{1} \leq r \leq r_{2}$, onde $r_{2}=-r_{1}$ é o outro ponto de retorno. Nesta região classicamente proibida, a partícula tunela através da barreira parabólica, pois a energia total é menor do que a barreira de potencial. A função de onda, pela aproximação WKB, pode então ser dada nesta região por uma expressão análoga à Eq. (D.14):

$$
\psi_{3}(r)=C_{3+} \sqrt{\frac{l(r)}{2 \pi}} e^{2 \pi \int_{r_{1}}^{r} \frac{\mathrm{d} x}{l(x)}}+C_{3-} \sqrt{\frac{l(r)}{2 \pi}} e^{-2 \pi \int_{r_{1}}^{r} \frac{\mathrm{d} x}{l(x)}}
$$

onde $C_{3+}$ e $C_{3-}$ são constantes e $l(r)$ é dado pela Eq. (D.15).

- Região 4: $r_{2} \leq r \leq r_{0}$. Nesta região, a partícula continua sujeita ao potencial parabólico mas, novamente, possui energia cinética maior que o potencial. A função de onda, pela aproximação WKB, pode então ser dada nesta região por uma expressão análoga à Eq. (D.23) da Região 2:

$$
\psi_{4}(r)=C_{4+} \sqrt{\frac{\lambda(r)}{2 \pi}} e^{2 \pi i \int_{r_{2}}^{r} \frac{\mathrm{d} x}{\lambda(x)}+i \pi / 4}+C_{4-} \sqrt{\frac{\lambda(r)}{2 \pi}} e^{-2 \pi i \int_{r_{2}}^{r} \frac{\mathrm{d} x}{\lambda(x)}-i \pi / 4}
$$

onde $C_{4+}$ e $C_{4-}$ são constantes e $\lambda(r)$ é o comprimento de onda local dado pela Eq. (D.5).

- Região 5: $r \geq r_{0}$. Finalmente, nesta região, a partícula executa movimento livre, na ausência do potencial parabólico. Logo, a correspondente função de onda nesta região, $\psi_{5}$, é descrita pela Eq. (D.2)

As fases $e^{ \pm i \pi / 4}$ foram escolhidas de forma a facilitar o uso das fórmulas de conexão ${ }^{1}$. De acordo com estas fórmulas de conexão, temos as seguintes relações:

$$
\begin{gathered}
C_{3+}=C_{2+}+C_{2-} \\
C_{3-}=\frac{i}{2}\left(C_{2-}-C_{2+}\right) \\
C_{3+} \beta_{+}=\frac{i}{2}\left(C_{4+}-C_{4-}\right)
\end{gathered}
$$

\footnotetext{
${ }^{1}$ As fórmulas de conexão são usadas quando, na aplicação do método WKB, existem pontos de retorno clássicos, onde o potencial possui o mesmo valor que a energia cinética assintótica. Vemos, pelas Eqs. (D.5) e (D.15), que, nas vizinhanças destes pontos de retorno, tanto $\lambda(x)$ como $l(x)$ divergem, exigindo cuidado na conexão entre as funções de onda dos dois lados de um ponto de retorno. As fórmulas de conexão fornecem relações entre os coeficientes das exponenciais de forma a minimizar este problema.
} 


$$
C_{3-} \beta_{-}=C_{4+}+C_{4-}
$$

onde $\beta_{ \pm}$são as integrais entre os pontos de retorno clássicos:

$$
\beta_{ \pm}=e^{ \pm 2 \pi \int_{r_{1}}^{r_{2}} \frac{\mathrm{d} x}{l(x)}}=e^{ \pm 2 \pi \frac{V_{B}-E}{2 \hbar \omega}}
$$

Usando as relações expressas nas Eqs. (D.26), (D.27), (D.28) e (D.29) podemos calcular as funções de onda em termos de apenas dois coeficientes, como, por exemplo, $C_{2+}$ e $C_{2-}$. Procedendo desta forma e aplicando as condições de continuidade das funções de onda $\psi_{1}, \psi_{2}, \psi_{3}, \psi_{4}$ e $\psi_{5}$ e suas respectivas derivadas nas interfaces entre as regiões (i.e., nos pontos $r=-r_{0}, r_{1}, r_{2}$ e $r_{0}$ ), obtemos o seguinte sistema de equações:

$$
\begin{aligned}
& A e^{i k r_{0}}=\frac{\sqrt{\hbar}}{\left[2 \mu\left(E-V_{B}+V_{B 0}\right)\right]^{1 / 4}}\left[C_{2+} e^{-i \phi_{1}}+C_{2-} e^{i \phi_{1}}\right] \\
& -i k A e^{i k r_{0}}=\frac{\hbar \omega}{4} \frac{\sqrt{V_{B 0}}}{\sqrt{\hbar}} \frac{\left[2 \mu\left(E-V_{B}+V_{B 0}\right)\right]^{1 / 4}}{\left(E-V_{B}+V_{B 0}\right)^{3 / 2}}\left[C_{2+} e^{-i \phi_{1}}+C_{2-} e^{i \phi_{1}}\right]+ \\
& +i \frac{\left[2 \mu\left(E-V_{B}+V_{B 0}\right)\right]^{1 / 4}}{\sqrt{\hbar}}\left[C_{2+} e^{-i \phi_{1}}-C_{2-} e^{i \phi_{1}}\right] \\
& e^{-i k r_{0}}+B e^{i k r_{0}}=\frac{\sqrt{\hbar}}{\left[2 \mu\left(E-V_{B}+V_{B 0}\right)\right]^{1 / 4}} \times \\
& \times\left\{i\left[\left(\frac{\beta_{-}}{4}-\beta_{+}\right) C_{2-}-\left(\frac{\beta_{-}}{4}+\beta_{+}\right) C_{2+}\right] e^{i \phi_{2}}+\right. \\
& \left.+i\left[\left(\frac{\beta_{-}}{4}+\beta_{+}\right) C_{2-}+\left(\beta_{+}-\frac{\beta_{-}}{4}\right) C_{2+}\right] e^{-i \phi_{2}}\right\} \\
& i k B e^{i k r_{0}}-i k e^{-i k r_{0}}=\frac{\left[2 \mu\left(E-V_{B}+V_{B 0}\right)\right]^{1 / 4}}{\sqrt{\hbar}}\left\{\left(\beta_{+}+\frac{\beta_{-}}{4}\right)\left[e^{i \phi_{2}} C_{2+}+e^{-i \phi_{2}} C_{2-}\right]+\right. \\
& \left.+\left(\beta_{+}-\frac{\beta_{-}}{4}\right)\left[e^{-i \phi_{2}} C_{2+}+e^{i \phi_{2}} C_{2-}\right]\right\}-i \frac{\hbar \omega}{4} \frac{\sqrt{V_{B 0}}}{\sqrt{\hbar}} \times \\
& \times \frac{\left[2 \mu\left(E-V_{B}+V_{B 0}\right)\right]^{1 / 4}}{\left(E-V_{B}+V_{B 0}\right)^{3 / 2}}\left\{\left(\beta_{+}+\frac{\beta_{-}}{4}\right)\left[e^{-i \phi_{2}} C_{2-}-e^{i \phi_{2}} C_{2+}\right]+\right. \\
& \left.+\left(\beta_{+}-\frac{\beta_{-}}{4}\right)\left[e^{-i \phi_{2}} C_{2+}-e^{i \phi_{2}} C_{2-}\right]\right\}
\end{aligned}
$$


onde as fases $\phi_{1}$ e $\phi_{2}$, são dadas por:

$$
\begin{aligned}
\phi_{1} & =2 \pi \int_{-r_{0}}^{r_{1}} \frac{\mathrm{d} x}{\lambda(x)}+\pi / 4 \\
& =\frac{V_{B}-E}{\hbar \omega} \ln \left[\frac{\sqrt{V_{B 0}}-\sqrt{E-V_{B}+V_{B 0}}}{\sqrt{V_{B}-E}}\right]+\frac{\sqrt{V_{B 0}\left(E-V_{B}+V_{B 0}\right)}}{\hbar \omega}+\frac{\pi}{4} \\
\phi_{2} & =2 \pi \int_{r_{2}}^{r_{0}} \frac{\mathrm{d} x}{\lambda(x)}+\pi / 4 \\
& =\frac{V_{B}-E}{\hbar \omega} \ln \left[\frac{\sqrt{V_{B}-E}}{\sqrt{V_{B 0}}+\sqrt{E-V_{B}+V_{B 0}}}\right]+\frac{\sqrt{V_{B 0}\left(E-V_{B}+V_{B 0}\right)}}{\hbar \omega}+\frac{\pi}{4}
\end{aligned}
$$

Resolvendo o sistema representado pelas Eqs. (D.31), (D.32), (D.33) e (D.34), obtemos, finalmente:

$$
\begin{aligned}
& C_{-}=\frac{8 i(2 \mu)^{1 / 4}}{\sqrt{\hbar}} \sqrt{E}\left(E-V_{B}+V_{B 0}\right)^{5 / 4} e^{-2 i \frac{\sqrt{E V_{B 0}}}{\hbar \omega}}\left\{\left(\beta_{+}-\frac{\beta_{-}}{4}\right) e^{i \phi_{2}} \times\right. \\
& \times\left[4\left(E-V_{B}+V_{B 0}\right)\left(\sqrt{E}-\sqrt{E-V_{B}+V_{B 0}}\right)-i \hbar \omega \sqrt{V_{B 0}}\right]\left[1+e^{2 i\left(\phi_{1}-\phi_{2}\right)}\right]+ \\
& -\frac{\left(\beta_{+}+\frac{\beta_{-}}{4}\right) e^{-i \phi_{2}}}{4\left(E-V_{B}+V_{B 0}\right)\left(\sqrt{E}+\sqrt{E-V_{B}+V_{B 0}}\right)-i \hbar \omega \sqrt{V_{B 0}}} \times \\
& \times\left(\left[4\left(E-V_{B}+V_{B 0}\right)\left(\sqrt{E}+\sqrt{E-V_{B}+V_{B 0}}\right)-i \hbar \omega \sqrt{V_{B 0}}\right]^{2}+\right. \\
& \left.\left.+e^{2 i\left(\phi_{1}+\phi_{2}\right)}\left[4\left(E-V_{B}+V_{B 0}\right)\left(\sqrt{E}-\sqrt{E-V_{B}+V_{B 0}}\right)-i \hbar \omega \sqrt{V_{B 0}}\right]^{2}\right)\right\}^{-1} \\
& C_{2+}=-C_{2-} e^{2 i \phi_{1}} \frac{4\left(E-V_{B}+V_{B 0}\right)\left(\sqrt{E}-\sqrt{E-V_{B}+V_{B 0}}\right)-i \hbar \omega \sqrt{V_{B 0}}}{4\left(E-V_{B}+V_{B 0}\right)\left(\sqrt{E}+\sqrt{E-V_{B}+V_{B 0}}\right)-i \hbar \omega \sqrt{V_{B 0}}}
\end{aligned}
$$

As amplitudes de transmissão $A$ e reflexão $B$ podem ser encontradas substituindo-se as Eqs. (D.37) e (D.38) nas Eq. (D.31) e (D.33). 


\section{Apêndice E}

\section{Potenciais de Polarização para Excitações}

\section{Coulombianas}

Desenvolvemos, aqui, a contribuição para o potencial de polarização de íons pesados para simular o efeito da excitação coulombiana do modo vibracional de octupolo de forma similar ao que foi feito décadas atrás para o acoplamento coulombiano do modo de quadrupolo vibracional e rotacional [101, 102]. O potencial de polarização descreve a absorção de fluxo do canal elástico, devido aos acoplamentos à estados excitados.

\section{E.1 Desenvolvimento Teórico}

Como na Ref. [102], consideramos a colisão de um núcleo esférico 1 com um núcleo-alvo deformável (núcleo 2), em energias próximas à altura da barreira. Consideramos a excitação coulombiana dos primeiros estados excitados de spin $I$ e número quântico magnético $M$ com energia de excitação $E_{I}$. Expandimos a função de onda no sistema de referência do centro de massa como

$$
|\psi\rangle=\frac{\sqrt{\pi}}{k_{I_{0}} r} \sum_{l_{0} l I J N}\left(2 l_{0}+1\right) \exp \left[i \sigma_{l_{0}}\left(\eta_{I_{0}}\right)\right]\left\langle l_{0} 0 I_{0} M_{0} \mid J N\right\rangle \psi_{l I J}^{l_{0} I_{0}}(r)|l I J N\rangle
$$


onde as funções de onda de canal são dadas por

$$
|l I J N\rangle=\sum_{m M}\langle l m I M \mid J N\rangle|I M\rangle Y_{l m}(\hat{r})
$$

Aqui $J$ é o momento angular total e $N$ é sua projeção no eixo $z$. Os índices $l_{0} I_{0}$ indicam o momento angular orbital inicial do projétil e spin do alvo respectivamente. Inserindo a função de onda na equação de Schrödinger independente do tempo, obtemos um sistema de equações acopladas para as funções de onda radiais para cada valor do momento angular total $J$ :

$$
\left(\frac{\mathrm{d}^{2}}{\mathrm{~d} r^{2}}+k_{I}^{2}-\frac{2 \mu}{\hbar^{2}} \frac{l(l+1)}{r^{2}}-\frac{2 \mu}{\hbar^{2}} \frac{Z_{1} Z_{2} e^{2}}{r}-\frac{2 \mu}{\hbar^{2}} V_{N}(r)\right) \psi_{l I J}^{l_{0} I_{0}}(r)=\sum_{l^{\prime} I^{\prime}} V_{l I, l^{\prime} I^{\prime}}^{J}(r) \psi_{l^{\prime} I^{\prime} J}^{l_{0} I_{0}}(r)
$$

O potencial de acoplamento para excitação coulombiana é dado por:

$$
\begin{aligned}
V_{l I, l^{\prime} I^{\prime}}^{J}(r)= & \frac{2 \mu}{\hbar^{2}} 2 \sqrt{\pi} Z_{1} e \sum_{\lambda}\left(\frac{(2 l+1)\left(2 l^{\prime}+1\right)}{(2 \lambda+1)}\right)^{1 / 2} \\
& \times(-1)^{J+I^{\prime}+\lambda}\left\langle I^{\prime}\|M(E \lambda)\| I\right\rangle\left(\begin{array}{ccc}
l & l^{\prime} & \lambda \\
0 & 0 & 0
\end{array}\right)\left\{\begin{array}{ccc}
J & l^{\prime} & I^{\prime} \\
\lambda & I & l
\end{array}\right\} \frac{1}{r^{\lambda+1}} \\
= & \sum_{\lambda} v_{l I, l^{\prime} I^{\prime}}^{J \lambda} \frac{1}{r^{\lambda+1}}
\end{aligned}
$$

onde $\left\langle I^{\prime}\|M(E \lambda)\| I\right\rangle$ é o elemento de matriz reduzida do momento de $\lambda$-polo elétrico do alvo. Definimos os coeficientes $v_{l I, l^{\prime} I^{\prime}}^{J \lambda}$ na expansão da Eq. (E.4) como:

$$
\begin{aligned}
v_{l I, l^{\prime} I^{\prime}}^{J \lambda}= & \frac{2 \mu}{\hbar^{2}} 2 \sqrt{\pi} Z_{1} e\left(\frac{(2 l+1)\left(2 l^{\prime}+1\right)}{(2 \lambda+1)}\right)^{1 / 2} \times \\
& \times(-1)^{J+I^{\prime}+\lambda}\left\langle I^{\prime}\|M(E \lambda)\| I\right\rangle\left(\begin{array}{lll}
l & l^{\prime} & \lambda \\
0 & 0 & 0
\end{array}\right)\left\{\begin{array}{lll}
J & l^{\prime} & I^{\prime} \\
\lambda & I & l
\end{array}\right\} .
\end{aligned}
$$

Os coeficientes são simétricos na troca de $l I$ e $l^{\prime} I^{\prime}$, logo a interação é hermitiana e real. No caso especial de uma transição de um estado fundamental $I=0^{+}$para um estado $I^{\prime}=\lambda$, os coeficientes 
se reduzem a:

$$
v_{l 0, l^{\prime} \lambda}^{l \lambda}=\frac{2 \mu}{\hbar^{2}} 2 \sqrt{\pi} Z_{1} e \frac{\sqrt{2 l^{\prime}+1}}{2 \lambda+1}(-1)^{l}\left(\begin{array}{ccc}
l & l^{\prime} & \lambda \\
0 & 0 & 0
\end{array}\right)\langle\lambda\|M(E \lambda)\| 0\rangle
$$

O potencial de polarização $V_{p o l}^{l_{0} I_{0} J}(r)$ é definido de forma que:

$$
V_{p o l}^{l_{0} I_{0} J}(r) \psi_{l_{0} I_{0} J}^{l_{0} I_{0}}(r)=\sum_{l^{\prime} I^{\prime}} V_{l I, l^{\prime} I^{\prime}}^{J}(r) \psi_{l^{\prime} I^{\prime} J}^{l_{0} I_{0}}(r)
$$

Podemos representar isto formalmente em termos de uma redução do tipo Feshbach das equações acopladas em $P=l_{0} I_{0}$ e $Q=l I \neq l_{0} I_{0}$. Usando o propagador no espaço $Q, G_{Q Q}\left(r, r^{\prime}\right)$, temos

$$
V_{p o l}(r)=\frac{1}{\psi_{P}(r)} V_{P Q}(r) \int_{0}^{\infty} d r^{\prime} G_{Q Q}\left(r, r^{\prime}\right) V_{Q P}\left(r^{\prime}\right) \psi_{P}\left(r^{\prime}\right)
$$

que podemos reescrever de forma mais explícita como

$$
V_{p o l}^{l_{0} I_{0} J}(r)=\frac{1}{\psi_{l_{0} I_{0} J}^{l_{0} I_{0}}(r)} \sum_{l I l^{\prime} I^{\prime}} V_{l_{0} I_{0}, l I}^{J}(r) \int_{0}^{\infty} d r^{\prime} G_{l I, l^{\prime} I^{\prime}}^{J}\left(r, r^{\prime}\right) V_{l^{\prime} I^{\prime}, l_{0} I_{0}}^{J}\left(r^{\prime}\right) \psi_{l_{0} I_{0} J}^{l_{0} I_{0}}\left(r^{\prime}\right)
$$

onde entendemos que as somas sobre $l I$ e $l^{\prime} I^{\prime}$ são restritas a valores diferentes de $l_{0} I_{0}$ e que o propagador $G_{l I, l^{\prime} I^{\prime}}^{J}\left(r, r^{\prime}\right)$ é restringido da mesma maneira.

Neste ponto, desejamos estimar as contribuições de ordem mais baixa para o potencial de polarização devido à excitação dos modos vibracionais de quadrupolo e octupolo. Restringimos, então, nossa atenção aos termos que acoplam o estado fundamental $I_{0}$ às vibrações de um fônon e de volta ao estado fundamental. Neste caso, podemos considerar o propagador como sendo o propagador coulombiano desacoplado, que aproximamos em seguida mantendo a parte do propagador que contém a função delta de Dirac de conservação de energia ${ }^{1}$ (parte imaginária):

$$
G_{l I, l^{\prime} I^{\prime}}^{J}\left(r, r^{\prime}\right)=-\frac{i}{k_{I}} F_{l}\left(k_{I} r_{<}\right) H_{l}^{(+)}\left(k_{I} r_{>}\right) \delta_{l l^{\prime}} \delta_{I I^{\prime}} \rightarrow-\frac{i}{k_{I}} F_{l}\left(k_{I} r\right) F_{l}\left(k_{I} r^{\prime}\right) \delta_{l l^{\prime}} \delta_{I I^{\prime}}
$$

\footnotetext{
${ }^{1}$ Isto é valido porque a interação coulombiana é relativamente fraca e a propagação é de fato quase conservativa.
} 
Tabela E.1: Os fatores semi-clássicos de perda de energia $g_{\lambda}(\xi)$ para excitações de quadrupolo e octupolo.

\begin{tabular}{|c|c|c|c|c|c|c|c|c|}
\hline$\xi$ & 0.0 & 0.2 & 0.4 & 0.6 & 0.8 & 1.0 & 1.2 & 1.4 \\
\hline \hline$g_{2}(\xi)$ & 1.0 & 0.815 & 0.452 & 0.206 & 0.084 & 0.032 & 0.011 & 0.004 \\
\hline$g_{3}(\xi)$ & 1.0 & 0.930 & 0.717 & 0.457 & 0.252 & 0.124 & 0.054 & 0.024 \\
\hline
\end{tabular}

onde $F\left(H^{(+)}\right)$é a função de onda regular (onda emergente). Também negligenciamos os efeitos do acoplamento e da interação nuclear na função de onda do canal elástico:

$$
\psi_{l_{0} I_{0} J}^{l_{0} I_{0}}(r)=F_{l_{0}}\left(k_{I_{0}} r\right)
$$

Podemos então escrever:

$$
V_{p o l}^{l_{0} I_{0} J}(r)=\frac{-i}{F_{l_{0}}\left(k_{I_{0}} r\right)} \sum_{\lambda l I} v_{l_{0} I_{0}, l I}^{J \lambda} \frac{1}{r^{\lambda+1}} F_{l}\left(k_{I} r\right) \frac{1}{k_{I}} v_{l I, l_{0} I_{0}}^{J \lambda} \int_{0}^{\infty} d r^{\prime} F_{l}\left(k_{I} r^{\prime}\right) \frac{1}{r^{\prime \lambda+1}} F_{l_{0}}\left(k_{I_{0}} r^{\prime}\right)
$$

Como simplificação seguinte, assumimos perda de energia nula nas funções de onda, com $k_{I} \rightarrow$ $k_{I_{0}}=k$, mas incluímos os fatores semi-clássicos de perda de energia, $g_{\lambda}\left(\xi_{I_{0} I}\right)$, da Ref. [103], que são dados por conveniência na Tabela E.1. Nesta tabela, $\xi$ é o parâmetro de adiabaticidade, $\xi=$ $\frac{1}{2} \eta E^{*} / E_{c m}$, onde $\eta$ é o parâmetro de Sommerfeld ${ }^{2}$ e $E^{*}$ é a energia de excitação correspondente. Com base na Tabela E.1, encontramos funções contínuas para $g_{\lambda}(\xi)$ :

$$
\begin{aligned}
& g_{2}(\xi)= \begin{cases}\mathrm{e}^{-4.963 \xi^{2}} & \text { para } \xi \leq 0.4 \\
0.452 e^{-4.4(\xi-0.4)} & \text { para } \xi>0.4\end{cases} \\
& g_{3}(\xi)= \begin{cases}\mathrm{e}^{-2.087 \xi^{2}} & \text { para } \xi \leq 1.0 \\
0.124 e^{-4.0(\xi-1.0)} & \text { para } \xi>1.0\end{cases}
\end{aligned}
$$

que foram usadas nos cálculos do Capítulo 6

\footnotetext{
${ }^{2} \mathrm{O}$ parâmetro de Sommerfeld é dado por $\eta=Z_{1} Z_{2} e^{2} / \sqrt{\frac{2 E_{c m} \hbar^{2}}{\mu}}$, onde $Z_{1}$ e $Z_{2}$ são os números atômicos do alvo e do projétil, respectivamente, e $e$ é a carga elétrica fundamental.
} 
Podemos, agora, escrever:

$$
V_{p o l}^{l_{0} I_{0} J}(r)=-i \sum_{\lambda l I} g_{\lambda}\left(\xi_{I_{0} I}\right)\left(v_{l_{0} I_{0}, l I}^{J \lambda}\right)^{2} M_{l l_{0}}^{\lambda} \frac{k^{2 \lambda}}{(k r)^{\lambda+1}} \frac{F_{l}(k r)}{F_{l_{0}}(k r)}
$$

onde

$$
M_{l l^{\prime}}^{\lambda}=\int_{0}^{\infty} d r F_{l}(r) \frac{1}{r^{\lambda+1}} F_{l^{\prime}}(r)
$$

As integrais também podem ser encontradas na Ref. [103]. Por conveniência, reproduzimo-as aqui:

$$
\begin{aligned}
M_{l l}^{2}= & \frac{1}{2 l(l+1)(2 l+1)}[2 l+1-\pi \eta+2 \eta \operatorname{Im} \psi(l+1+i \eta)] \\
M_{l l+2}^{2}= & \frac{1}{6} \frac{1}{|l+1+i \eta||l+2+i \eta|} \\
M_{l l+1}^{3}= & \frac{1}{2 l(l+1)(l+2)(2 l+1)(2 l+3)|l+1+i \eta|} \\
& \times\left\{3|l+1+i \eta|^{2}[2 l+1-\pi \eta+2 \eta \operatorname{Im} \psi(l+1+i \eta)]-l(l+1)(2 l+1)\right\} \\
M_{l l+3}^{3}= & \frac{1}{15} \frac{1}{|l+1+i \eta||l+2+i \eta||l+3+i \eta|}
\end{aligned}
$$

onde a parte imaginária da função di-gama $\operatorname{Im} \psi$ é dada por:

$$
\begin{aligned}
\operatorname{Im} \psi(l+1+i \eta) & =\frac{\pi}{2} \operatorname{coth} \pi \eta+\frac{1}{2 \eta}-\eta \sum_{n=0}^{l} \frac{1}{n^{2}+\eta^{2}} \\
& =\frac{\pi}{\exp (2 \pi \eta)-1}+\frac{\pi}{2}+\frac{1}{2 \eta}-\eta \sum_{n=0}^{l} \frac{1}{n^{2}+\eta^{2}}
\end{aligned}
$$

Notamos que o termo da exponencial em $\eta$ é extremamente pequeno para $\eta \geq 1$ e o desprezamos aqui. Substituindo a Eq. (E.21) nos elementos de matriz, representados pelas Eqs. (E.17) a (E.20), 
podemos reescrevê-los como:

$$
\begin{aligned}
M_{l l}^{2}= & \frac{1}{l(l+1)(2 l+1)}\left[l+1-\eta^{2} \sum_{n=0}^{l} \frac{1}{n^{2}+\eta^{2}}\right] \\
M_{l l+2}^{2}= & \frac{1}{6} \frac{1}{\sqrt{\left((l+1)^{2}+\eta^{2}\right)\left((l+2)^{2}+\eta^{2}\right)}} \\
M_{l l+1}^{3}= & \frac{1}{2 l(l+1)(l+2)(2 l+1)(2 l+3) \sqrt{(l+1)^{2}+\eta^{2}}} \\
& \times\left\{6\left((l+1)^{2}+\eta^{2}\right)\left[l+1-\eta^{2} \sum_{n=0}^{l} \frac{1}{n^{2}+\eta^{2}}\right]-l(l+1)(2 l+1)\right\} \\
M_{l l+3}^{3}= & \frac{1}{15} \frac{1}{\sqrt{\left((l+1)^{2}+\eta^{2}\right)\left((l+2)^{2}+\eta^{2}\right)\left((l+3)^{2}+\eta^{2}\right)}} .
\end{aligned}
$$

Com expressões para os símbolos $3-j$ e $6-j$ e para as funções de onda de Coulomb $F_{l}(k r)$, poderíamos, agora, calcular o potencial de polarização. No entanto, para obtermos uma expressão de forma fechada de alguma forma mais simples, assumimos que o estado fundamental do alvo é um estado $0^{+}$e usamos as mesmas aproximações para grandes valores de momento angular orbital usadas nas Refs. [101, 102]. Podemos, então, escrever os elementos de matriz de coulombianos como

$$
\begin{aligned}
M_{l l}^{2} & \approx \frac{1}{2 \bar{l}^{2}}\left(1-\frac{\eta}{\bar{l}} \arctan \frac{\bar{l}}{\eta}\right) \\
M_{l l \pm 2}^{2} & \approx \frac{1}{6} \frac{1}{\bar{l}^{2}+\eta^{2}} \\
M_{l l \pm 1}^{3} & \approx \frac{1}{4 \bar{l}^{4}}\left[3 \sqrt{\bar{l}^{2}+\eta^{2}}\left(1-\frac{\eta}{\bar{l}} \arctan \frac{\bar{l}}{\eta}\right)-\frac{\bar{l}^{2}}{\sqrt{\bar{l}^{2}+\eta^{2}}}\right] \\
M_{l l \pm 3}^{3} & \approx \frac{1}{15} \frac{1}{\left(\bar{l}^{2}+\eta^{2}\right)^{3 / 2}},
\end{aligned}
$$


$\operatorname{com} \bar{l}=l+1 / 2$. Os símbolos $3-j$ relevantes são

$$
\begin{aligned}
\left(\begin{array}{lll}
l & l & 2 \\
0 & 0 & 0
\end{array}\right) & =(-1)^{l+1}\left[\frac{l(l+1)}{(2 l-1)(2 l+1)(2 l+3)}\right]^{1 / 2} \approx(-1)^{l+1} \frac{1}{\sqrt{8 l}} \\
\left(\begin{array}{ccc}
l & l+2 & 2 \\
0 & 0 & 0
\end{array}\right) & =(-1)^{l}\left[\frac{3}{2} \frac{(l+1)(l+2)}{(2 l+1)(2 l+3)(2 l+5)}\right]^{1 / 2} \approx(-1)^{l} \sqrt{\frac{3}{16 l}} \\
\left(\begin{array}{ccc}
l & l+1 & 3 \\
0 & 0 & 0
\end{array}\right) & =(-1)^{l}\left[\frac{3}{2} \frac{l(l+1)(l+2)}{(2 l-1)(2 l+1)(2 l+3)(2 l+5)}\right]^{1 / 2} \approx(-1)^{l} \sqrt{\frac{3}{32 l}} \\
\left(\begin{array}{ccc}
l & l+3 & 3 \\
0 & 0 & 0
\end{array}\right) & =(-1)^{l+1}\left[\frac{5}{2} \frac{(l+1)(l+2)(l+3)}{(2 l+1)(2 l+3)(2 l+5)(2 l+7)}\right]^{1 / 2} \approx(-1)^{l+1} \sqrt{\frac{5}{32 l}}
\end{aligned}
$$

de forma que podemos escrever os coeficientes $v$ ao quadrado como:

$$
\begin{aligned}
\left(v_{l 0, l 2}^{l 2}\right)^{2} & \approx \pi\left(\frac{2 \mu}{\hbar^{2}} Z_{1} e\right)^{2} B(E 2) \uparrow \frac{1}{25} \\
\left(v_{l 0, l \pm 22}^{l 2}\right)^{2} & \approx \pi\left(\frac{2 \mu}{\hbar^{2}} Z_{1} e\right)^{2} B(E 2) \uparrow \frac{3}{2 \cdot 25} \\
\left(v_{l 0, l \pm 13}^{l 3}\right)^{2} & \approx \pi\left(\frac{2 \mu}{\hbar^{2}} Z_{1} e\right)^{2} B(E 3) \uparrow \frac{3}{4 \cdot 49} \\
\left(v_{l 0, l \pm 33}^{l 3}\right)^{2} & \approx \pi\left(\frac{2 \mu}{\hbar^{2}} Z_{1} e\right)^{2} B(E 3) \uparrow \frac{5}{4 \cdot 49} .
\end{aligned}
$$

Se agora substituirmos os coeficientes, expressos nas Eqs. (E.34) a (E.37), e os elementos de matriz, representados pelas Eqs. (E.26) a (E.29), em nossa expressão para o potencial de polarização, Eq. (E.15), encontraremos:

$$
\begin{aligned}
V_{p o l}^{l}(r)= & -i \pi\left(\frac{2 \mu}{\hbar^{2}} Z_{1} e k^{2}\right)^{2} \frac{B(E 2) \uparrow}{50} \frac{g_{2}\left(\xi_{02}\right)}{(k r)^{3}}\left\{\frac{1}{\bar{l}^{2}}\left(1-\frac{\eta}{\bar{l}} \arctan \frac{\bar{l}}{\eta}\right)\right. \\
& \left.+\frac{1}{2} \frac{1}{\bar{l}^{2}+\eta^{2}}\left(\frac{F_{l+2}(k r)}{F_{l}(k r)}+\frac{F_{l-2}(k r)}{F_{l}(k r)}\right)\right\} \\
& -i \pi\left(\frac{2 \mu}{\hbar^{2}} Z_{1} e k^{3}\right)^{2} \frac{B(E 3) \uparrow}{196} \frac{g_{3}\left(\xi_{03}\right)}{(k r)^{4}}\left\{\frac{1}{3} \frac{1}{\left(\bar{l}^{2}+\eta^{2}\right)^{3 / 2}}\left(\frac{F_{l+3}(k r)}{F_{l}(k r)}+\frac{F_{l-3}(k r)}{F_{l}(k r)}\right)\right. \\
& \left.+\frac{3}{4 \bar{l}^{4}}\left[3 \sqrt{\bar{l}^{2}+\eta^{2}}\left(1-\frac{\eta}{\bar{l}} \arctan \frac{\bar{l}}{\eta}\right)-\frac{\bar{l}^{2}}{\sqrt{\bar{l}^{2}+\eta^{2}}}\right]\left(\frac{F_{l+1}(k r)}{F_{l}(k r)}+\frac{F_{l-1}(k r)}{F_{l}(k r)}\right)\right\}
\end{aligned}
$$


Para simplificar as razões das funções de onda coulombianas, usamos a relação de recursividade:

$$
(2 l+1)\left[\eta+\frac{l(l+1)}{k r}\right] F_{l}(k r)=l \sqrt{(l+1)^{2}+\eta^{2}} F_{l+1}(k r)+(l+1) \sqrt{l^{2}+\eta^{2}} F_{l-1}(k r),
$$

que pode ser escrita, para valores grandes de momento angular, como

$$
2\left[\eta+\frac{\bar{l}^{2}}{k r}\right] F_{l}(k r) \approx \sqrt{\bar{l}^{2}+\eta^{2}}\left(F_{l+1}(k r)+F_{l-1}(k r)\right) .
$$

A Eq. (E.40) leva imediatamente a:

$$
\frac{F_{l+1}(k r)}{F_{l}(k r)}+\frac{F_{l-1}(k r)}{F_{l}(k r)} \approx \frac{2}{\sqrt{\bar{l}^{2}+\eta^{2}}}\left[\eta+\frac{\bar{l}^{2}}{k r}\right]
$$

Se escrevermos a relação de recursividade como uma equação para $F_{l}$ e substituirmos para as funções $F_{l+1}$ e $F_{l-1}$ do lado direito, obtemos:

$$
\frac{F_{l+2}(k r)}{F_{l}(k r)}+\frac{F_{l-2}(k r)}{F_{l}(k r)} \approx-2+\frac{4}{\bar{l}^{2}+\eta^{2}}\left(\eta+\frac{\bar{l}^{2}}{k r}\right)^{2}
$$

Repetindo a operação, obtemos:

$$
\frac{F_{l+3}(k r)}{F_{l}(k r)}+\frac{F_{l-3}(k r)}{F_{l}(k r)}+3\left(\frac{F_{l+1}(k r)}{F_{l}(k r)}+\frac{F_{l-1}(k r)}{F_{l}(k r)}\right) \approx \frac{8}{\left(\bar{l}^{2}+\eta^{2}\right)^{3 / 2}}\left(\eta+\frac{\bar{l}^{2}}{k r}\right)^{3}
$$

ou

$$
\frac{F_{l+3}(k r)}{F_{l}(k r)}+\frac{F_{l-3}(k r)}{F_{l}(k r)} \approx \frac{8}{\left(\bar{l}^{2}+\eta^{2}\right)^{3 / 2}}\left(\eta+\frac{\bar{l}^{2}}{k r}\right)^{3}-\frac{6}{\sqrt{\bar{l}^{2}+\eta^{2}}}\left(\eta+\frac{\bar{l}^{2}}{k r}\right) .
$$

Substituindo estas equações na expressão para o potencial de polarização, Eq. (E.38), temos, final- 
mente:

$$
\begin{aligned}
V_{\text {pol }}^{l}(r)= & -i \pi\left(\frac{2 \mu}{\hbar^{2}} Z_{1} e k^{2}\right)^{2} \frac{B(E 2) \uparrow}{50} \frac{g_{2}\left(\xi_{02}\right)}{(k r)^{3}} \times \\
& \times\left\{\frac{1}{\bar{l}^{2}}\left(\frac{\eta^{2}}{\overline{l^{2}}+\eta^{2}}-\frac{\eta}{\bar{l}} \arctan \frac{\bar{l}}{\eta}\right)+\frac{2}{\left(\bar{l}^{2}+\eta^{2}\right)^{2}}\left(\eta+\frac{\bar{l}^{2}}{k r}\right)^{2}\right\} \\
& -i \pi\left(\frac{2 \mu}{\hbar^{2}} Z_{1} e k^{3}\right)^{2} \frac{B(E 3) \uparrow}{196} \frac{g_{3}\left(\xi_{03}\right)}{(k r)^{4}} \times \\
& \times\left\{\frac{8}{3} \frac{1}{\left(\bar{l}^{2}+\eta^{2}\right)^{3}}\left(\eta+\frac{\bar{l}^{2}}{k r}\right)^{3}\right. \\
& \left.+\frac{3}{2 \bar{l}^{4}}\left[3\left(1-\frac{\eta}{\bar{l}} \arctan \frac{\bar{l}}{\eta}\right)-\frac{\bar{l}^{2}}{\bar{l}^{2}+\eta^{2}}-\frac{4}{3} \frac{\bar{l}^{4}}{\left(\bar{l}^{2}+\eta^{2}\right)^{2}}\right]\left(\eta+\frac{\bar{l}^{2}}{k r}\right)\right\}
\end{aligned}
$$

Para concluir, este potencial de polarização está em unidades de inverso de comprimento ao quadrado. O potencial em unidades de energia pode ser obtido multiplicando-se a Eq. (E.45) por $\frac{\hbar^{2}}{2 \mu}$. 


\section{Referências Bibliográficas}

[1] A. B. Balantekin, N. Takigawa, Rev. Mod. Phys. 70, 77 (1998).

[2] L. C. Vaz, J. M. Alexander, Phys. Rev. C 18, 2152 (1978).

[3] R. Vandenbosch, Annu. Rev. Nucl. Sci. 42, 447 (1992).

[4] A. M. Stefanini et al., in Proceedings of the Workshop on Heavy Ion Fusion: Exploring the Variety of Nuclear Properties, Padova, Italy (1994), World Scientific Press, Singapore 1994.

[5] N. Rowley, G. R. Satchler, P. H. Stelson, Phys. Lett. B 314, 179 (1991).

[6] A. M. Stefanini et al, Phys. Rev. C 73, 034606 (2006).

[7] J. O. Newton, R. D. Butt, M. Dasgupta, D. J. Hinde, I. I. Gontchar, C. R. Morton, K. Hagino, Phys. Lett. B 586, 219 (2004).

[8] C. L. Jiang et al, Phys. Rev. Lett. 93, 012701 (2004).

[9] C. L. Jiang et al, Phys. Rev. Lett. 89, 052701 (2002).

[10] K. Hagino, N. Rowley, M. Dasgupta, Phys. Rev. C 67, 054603 (2003).

[11] H. Esbensen, Phys. Rev. C 72, 054607 (2005).

[12] H. Esbensen, Nucl. Phys. A 352, 147 (1981).

[13] H. Esbensen, Jian-Qun Wu, G. F. Bertsch, Nucl. Phys. A 411 (1983) 275.

[14] C. H. Dasso, S. Landowne, A. Winther, Nucl. Phys. A 407, 221 (1983).

[15] N. Takigawa, G. F. Bertsch, Phys. Rev. C 29 (1984) 2358.

[16] K. Hagino, N. Takigawa, J. R. Bennett, D. M. Brink, Phys. Rev. C 51 (1995) 3190.

[17] M. A. Candido Ribeiro, L. C. Chamon, D. Pereira, M. S. Hussein, D. Galetti, Phys. Rev. Lett. 78, 3270 (1997). 
[18] L. C. Chamon, D. Pereira, M. S. Hussein, M. A. Candido Ribeiro, D. Galetti, Phys. Rev. Lett. 79, 5218 (1997).

[19] L. C. Chamon, B. V. Carlson, L. R. Gasques, D. Pereira, C. De Conti, M. A. G. Alvarez, M. S. Hussein, M. A. Candido Ribeiro, E. S. Rossi Jr., C. P. Silva, Phys. Rev. C 66, 014610 (2002).

[20] J. J. S. Alves, P. R. S. Gomes, J. Lubian, L. C. Chamon, D. Pereira, R. M. Anjos, E. S. Rossi, Jr., C. P. Silva, M. A. G. Alvarez, G. P. A. Nobre, L. R. Gasques, Nucl. Phys. A 748 (2005) 59.

[21] D. Pereira, C. P. Silva, J. Lubian, E. S. Rossi Jr., L. C. Chamon, Phys. Rev. C 73 (2006) 014601.

[22] L. C. Chamon, D. Pereira, M. S. Hussein, Phys. Rev. C 58, 576 (1998).

[23] M. A. G. Alvarez, L. C. Chamon, D. Pereira, E. S. Rossi Jr., C. P. Silva, L. R. Gasques, H. Dias, M. O. Roos, Nucl. Phys. A 656, 187 (1999).

[24] L. R. Gasques, L. C. Chamon, C. P. Silva, D. Pereira, M. A. G. Alvarez, E. S. Rossi Jr., V. P. Likhachev, B. V. Carlson, C. De Conti, Phys. Rev. C 65, 044314 (2002).

[25] E. S. Rossi Jr., D. Pereira, L. C. Chamon, C. P. Silva, M. A. G. Alvarez, L. R. Gasques, J. Lubian, B. V. Carlson, C. De Conti, Nucl. Phys. A 707, 325 (2002).

[26] L. R. Gasques et al, Phys. Rev. C 67, 024602 (2003).

[27] T. Tarutina, L. C. Chamon, M. S. Hussein, Phys. Rev. C 67, 044605 (2003).

[28] M. A. G. Alvarez, L. C. Chamon, M. S. Hussein, D. Pereira, L. R. Gasques, E. S. Rossi Jr., C. P. Silva, Nucl. Phys. A 723, 93 (2003).

[29] L. R. Gasques, L. C. Chamon, D. Pereira, M. A. G. Alvarez, E. S. Rossi Jr., C. P. Silva, G. P. A. Nobre, B. V. Carlson, Phys. Rev. C 67, 067603 (2003).

[30] P. R. S. Gomes et al, Phys. Rev. C 70, 054605 (2004).

[31] M. A. G. Alvarez, N. Alamanos, L. C. Chamon, M. S. Hussein, Nucl. Phys. A 753, 83 (2005).

[32] P. R. S. Gomes et al, Phys. Rev. C 71, 034608 (2005).

[33] no.3b 909 (2005). J. J. S. Alves, P. R. S. Gomes, J. Lubian, L. C. Chamon, R. M. Anjos, D. Pereira, E. S. Rossi, Jr., C. P. Silva, M. A. G. Alvarez, G. P. A. Nobre, L. R. Gasques, Braz. J. Phys. 35.

[34] P. R. S. Gomes et al, J. Phys. G 31, S1669 (2006).

[35] P. R. S. Gomes et al, Phys. Lett. B 634, 356 (2006). 
[36] G. P. A. Nobre, L. C. Chamon, L. R. Gasques, B. V. Carlson, I. J. Thompson, Physical Review C 75, 044606 (2007).

[37] G. P. A. Nobre, L. C. Chamon, B. V. Carlson, I. J. Thompson, L. R. Gasques, Nuclear Physics A 786, 90-106 (2007).

[38] L. C. Chamon, G. P. A. Nobre, D. Pereira, E. S. Rossi Jr., C. P. Silva, L. R. Gasques, B. V. Carlson, Phys. Rev. C 70, 014604 (2004).

[39] D. Pereira, E. S. Rossi, Jr., G. P. A. Nobre, L. C. Chamon, C. P. Silva, L. R. Gasques, M. A. G. Alvarez, R. V. Ribas, J. R. B. Oliveira, N. H. Medina, M. N. Rao, E. W. Cybulska, W. A. Seale, N. Carlin, P. R. S. Gomes, J. Lubian, R. M. Anjos, Phys. Rev. C 74 (2006) 034608.

[40] L. C. Chamon, G. P. A. Nobre, D. Pereira, C. P. Silva, E. S. Rossi, Jr., L. R. Gasques, B. V. Carlson, Braz. J. Phys. 35, no.3b 906-908 (2005).

[41] G. P. A. Nobre, L. C. Chamon, B. V. Carlson, Polarization potential for the heavy-ion elastic scattering process submetido à Nuclear Physics $\mathbf{A}$.

[42] G. P. A. Nobre, C. P. Silva, L. C. Chamon, B. V. Carlson, Consistent analysis of fusion data without adjustable parameters for systems involving odd nuclei submetido à Physical Review C.

[43] D. L. Hill, J. A. Wheeler, Phys. Rev. 89 (1953) 1102.

[44] G. R. Satchler, W. G. Love, Phys. Rep. 55, 183 (1979).

[45] H. J. Krappe, Ann. of Phys. 99, 142 (1976).

[46] N. Takigawa, T. Rumin, N. Ihara, Phys.Rev. C 61, 044607 (2000).

[47] M. Ismail, A. Y. Ellithi, F. Salah, Phys. Rev. C 66, 017601 (2002).

[48] H. De Vries, C. W. De Jager, C. De Vries, At. Data Nucl. Data Tables 36, 495 (1987).

[49] B. V. Carlson, D. Hirata, Phys. Rev. C 62, 054310 (2000).

[50] S. Raman, C. W. Nestor Jr., P. Tikkanen, At. Data and Nuclear Data Tables 78, 1 (2001).

[51] T. Kibedi, R. H. Spear, At. Data and Nuclear Data Tables 80, 35 (2002).

[52] A. R. Edmonds, Angular Momentum in Quantum Mechanics, Princeton Univ. Press, Princeton (1960).

[53] B. V. Carlson, L. C. Chamon, L. R. Gasques, Phys. Rev. C 70, 057602 (2004). 
[54] M. A. G. Alvarez et al, Phys. Rev. C 65, 014602 (2002).

[55] L. R. Gasques, L. C. Chamon, D. Pereira, M. A. G. Alvarez, E. S. Rossi Jr., C. P. Silva, B. V. Carlson, Phys. Rev. C 69, 034603 (2004).

[56] D. Ackermann et al, Nucl. Phys. A 609 (1996) 91.

[57] M. Beckerman, M. Salomaa, A. Sperduto, J. D. Molitoris, A. DiRienzo, Phys. Rev. C 25 (1982) 837.

[58] R. G. Stokstad et al, Phys. Rev. Lett. 41 (1978) 475.

[59] R. G. Stokstad et al, Z. Phys. A 295 (1980) 269.

[60] W. Reisdorf et al, Phys. Rev. Lett. 49 (1982) 1811.

[61] M. Beckerman, J. Ball, H. Enge, M. Salomaa, A. Sperduto, S. Gazes, A. DiRienzo, J. D. Molitoris, Phys. Rev. C 23, 1581 (1981).

[62] H. Esbensen, S. Landowne, Phys. Rev. C 35, 2090 (1987).

[63] C. H. Dasso, S. Landowne, Z. Phys. A 322, 175 (1985).

[64] I. J. Thompson, Comp. Phys. Rep. 7, 167 (1988).

[65] S. Raman, C. H. Malarkey, W. T. Milner, C. W. Nestor Jr., P. H. Stelson, At. Data Nucl. Data Tab. 36, 1 (1987).

[66] M. G. Mazarakis, W. E. Stephens, Phys. Rev. C 7, 1280 (1973).

[67] M. D. High, B. Cujec, Nucl. Phys. A 282, 181 (1977).

[68] J. R. Patterson, H. Winkler, C. S. Zaidnis, Astrophys. Jour. 157, 367 (1969).

[69] D. G. Kovar et al, Phys. Rev. C 20, 1305 (1979).

[70] P. Rosales et al, Revista Mexicana de Física 49, 88 (2003).

[71] J. Thomas, Y. T. Chen, S. Hinds, D. Meredith, M. Olson, Phys. Rev. C 33, 1679 (1986).

[72] G. Hulke, C. Rolfs, H. P. Trautvetter, Z. Phys. A 297, 161 (1980).

[73] A. Kuronen, J. Keinonen, P. Tikkanen, Phys. Rev. C 35, 591 (1986).

[74] S. C. Wu, C. A. Barnes, Nucl. Phys. A 422, 373 (1983). 
[75] J. O. Newton, C. R. Morton, M. Dasgupta, J. R. Leigh, J. C. Mein, D. J. Hinde, H. Timmers, K. Hagino, Phys. Rev. C 64, 064608 (2001).

[76] S. Santra, P. Singh, S. Kailas, A. Chatterjee, A. Shrivastava, K. Mahata, Phys. Rev. C 64, $024602(2001)$.

[77] E. Vulgaris, L. Grodzins, S. G. Steadman, R. Ledoux, Phys. Rev. C 33, 2017 (1986).

[78] H. Timmers, Ph.D thesis, The Australian National University (1996).

[79] J. R. Leigh et al, Phys. Rev. C 52, 3151 (1995).

[80] C. R. Morton, A. C. Berriman, M. Dasgupta, D. J. Hinde, J. O. Newton, K. Hagino, I. J. Thompson, Phys. Rev. C 60, 044608 (1999).

[81] S. Mitsuoka, H. Ikezoe, K. Nishio, J. Lu, Phys. Rev. C 62, 054603 (2000).

[82] D. J. Hinde, W. Pan, A. C. Berriman, R. D. Butt, M. Dasgupta, C. R. Morton, J. O. Newton, Phys. Rev. C 62, 024615 (2000).

[83] R. G. Stokstad, Y. Eisen, S. Kaplanis, D. Pelte, U. Smilansky, I. Tserruya, Phys. Rev. C 21, 2427 (1980).

[84] M. Dasgupta, A. Navin, Y. K. Agarwal, C. V. K. Baba, H. C. Jain, M. L. Jhingan, A. Roy, Nucl. Phys. A 539, 351 (1992).

[85] M. Dasgupta et al, Proceedings of the Workshop on Heavy-ion Fusion: Exploring the Variety of Nuclear Proprieties, Padova, Italy (1994), World Scientific Press, Singapore, 1994.

[86] R. Pengo, D. Evers, K. E. G. Löbner, U. Quade, K. Rudolph, S. J. Skorka, I. Weidl, Nucl. Phys. A 411, 255 (1983).

[87] A. M. Stefanini, L. Corradi, A. M. Vinodkumar, Yang Feng, F. Scarlassara, G. Montagnoli, S. Beghini, M. Bisogno, Phys. Rev. C 62, 014601 (2000).

[88] H. A. Aljuwair, R. J. Ledoux, M. Beckerman, S. B. Gazes, J. Wiggins, E. R. Cosman, R. R. Betts, S. Saini, Ole Hansesn, Phys. Rev. C 30, 1223 (1984).

[89] A. A. Sonzogni, J. D. Bierman, M. P. Kelly, J. P. Lestone, J. F. Liang, R. Vandenbosch, Phys. Rev. C 57, 722 (1998).

[90] J. D. Bierman, P. Chan, J. F. Liang, M. P. Kelly, A. A. Sonzogni, R. Vandenbosch, Phys. Rev. C 54, 3068 (1996). 
[91] H. Timmers, D. Ackermann, S. Beghini, L. Corradi, J. H. He, G. Montagnoli, F. Scarlassara, A. M. Stefanini, N. Rowley, Nucl. Phys. A 633, 421 (1998).

[92] W. Reisdorf et al, Nucl. Phys. A 438, 212 (1985).

[93] W. Reisdorf, F. P. Hessberger, K. D. Hildenbrand, S. Hofmann, G. Münzenberg, K. H. Schmidt, W. F. W. Schneider, K. Sümmerer, G. Wirth, J. V. Kratz, K. Schutt, C. C. Sahm, Nucl. Phys. A 444, 154 (1985).

[94] J. G. Keller, K. -H Schmidt, F. P. Hessberger, G. Munzenberg, W. Reisdorf, H. -G. Clerc, C. -C. Sahm, Nucl. Phys. A 452, 173 (1986).

[95] F. L. H. Wolfs, Phys. Rev. C 36, 1379 (1987).

[96] C. L. Jiang et al, Phys. Rev. C 71, 044613 (2005).

[97] D. J. Hinde, M. Dasgupta, J. R. Leigh, J. C. Mein, C. R. Morton, J. O. Newton, H. Timmers, Phys. Rev. C 53, 1290 (1996).

[98] D. J. Hinde, C. R. Morton, M. Dasgupta, J. R. Leigh, J. C. Mein, H. Timmers, Nucl. Phys. A 592, 271 (1995).

[99] A. M. Stefanini, D. Ackermann, L. Corradi, J. H. He, G. Montagnoli, S. Beghini, F. Scarlassara, G. F. Segato, Phys. Rev. C 52, R1727 (1995).

[100] F. Scarlassara, S. Beghini, G. Montagnoli, G. F. Segato, D. Ackerman, L. Corradi, C. J. Lin, A. M. Stefanini, L. F. Zheng, Nucl. Phys. A 672, 99 (2000).

[101] A. J. Baltz, N. K. Glendenning, S. K. Kaufmann, K. Pruess, Nucl. Phys. A 327, 221 (1979).

[102] M. S. Hussein, A. J. Baltz, B. V. Carlson, Phys. Rep. 113, 133 (1984).

[103] K. Alder, A. Bohr, T. Huus, B. Mottelson, A. Winther, Rev. Mod. Phys. 28, 1432 (1956).

[104] B. Dasmahapatra, B. Cujec, F. Lahlou, Can. J. Phys. 61, 657 (1983).

[105] D. M. Nadkarni, A. Saxena, D. C. Biswas, R. K. Choudhury, S. S. Kapoor, Phys. Rev. C 59, R580 (1999).

[106] A . Mukherjee et al, Phys. Lett. B 636, 91 (2006).

[107] P. R. S. Gomes, T. J. P. Penna, E. F. Chagas, R. Liguori Neto, J. C. Acquadro, P. R. Pascholati, N. Carlin, M. M. Coimbra, Nucl. Phys. A 534, 429 (1991). 
[108] D. J. Hinde, A. C. Berriman, M. Dasgupta, J. R. Leigh, J. C. Mein, C. R. Morton, J. O. Newton, Phys. Rev. C 60, 054602 (1999).

[109] L. C. Chamon, D. Pereira, E. S. Rossi, C. P. Silva, G. R. Razeto, A. M. Borges, L. C. Gomes, O. Sala, Phys. Lett. B 275, 29 (1992).

[110] E. F. Aguilera, J. J. Vega, J. J. Kolata, A. Morsad, R. G. Tighe, X. J. Kong, Phys. Rev. C 41, 910 (1990).

[111] P. H. Stelson, H. J. Kim, M. Beckerman, D. Shapira, R. L. Robinson, Phys. Rev. C 41, 1584 (1990).

[112] W. Scobel, H. H. Gutbrod, M. Blann, A. Mignerey, Phys. Rev. C 14, 1808 (1976).

[113] S. J. Skorka et al, Z. Phys. A 328, 355 (1987).

[114] J. J. Vega, E. F. Aguilera, G. Murillo, J. J. Kolata, A. Morsad, X. J. Kong, Phys. Rev. C 42, 947 (1990).

[115] P. David, J. Bisplinghoff, M. Blann, T. Mayer-Kuckuk, A. Mignerey, Nucl. Phys. A 287, 179 (1977).

[116] E. Crema, L. C. Chamon, P. R. S. Gomes, Phys. Rev. C 72, 034610 (2005).

[117] M. Dasgupta et al, Phys. Rev. Lett. 82, 1395 (1999).

[118] C. Beck et al, Phys. Rev. C 67, 054602 (2003).

[119] M. Dasgupta et al, Phys. Rev. C 70, 024606 (2004).

[120] Vandana Tripathi, A. Navin, K. Mahata, K. Ramachandran, A. Chatterjee, S. Kailas, Phys. Rev. Lett. 88, 172701 (2002).

[121] Y. W. Wu, Z. H. Liu, C. J. Lin, H. Q. Zhang, M. Raun, F. Yang, Z. C. Li, M. Trotta, K. Hagino, Phys. Rev. C 68, 044605 (2003).

[122] Handbook of Mathematical Functions, edited by M. Abramowitz and I. A. Stegun, New York: Dover Publications, section 19 (1965).

[123] A. F. R. T. Piza, Mecânica Quântica, Editora da Universidade de São Paulo - Edusp, São Paulo (2002). 